\title{
The Blood Picture in Hemorrhagic Anemia
}

\author{
Jane M. Leichsenring and Alice Biester \\ With the Collaboration of
}

Hortense Hönig Deinard, Anita S. Longley, Sparkle M. Furnas, Elisabeth Sutherland, Edith S. Foss, Florence Sperry,

Virginia R. Roe, and Lida M. Burrill

Division of Home Economics

she

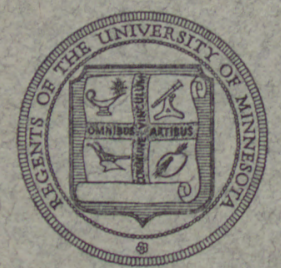

University of Minnesota Agricultural Experiment Station 


\title{
The Blood Picture in Hemorrhagic Anemia
}

Jane M. Leichsenring and Alice Biester

With the Collaboration of

Hortense Hönig Deinard, Anita S. Longley, Sparkle M. Furnas,

Elisabeth Sutherland, Edith S. Foss, Florence Sperry,

Virginia R. Roe, and Lida M. Burrill

Division of Home Economics

\author{
University of Minnesota \\ Agricultural Experiment Station
}

Accepted for publication June 2, 1939. 
Table of contents ………................................................................................................................ 3

List of tables ........................................................................................................... 5

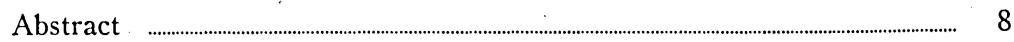

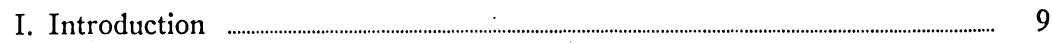

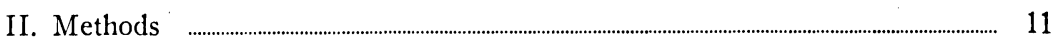

Selection and care of experimental animals ......................................................... 11

Synthetic diet _..................................................................................................................... 12

Experimental procedure ...................................................................................................... 12

Physical measurements on blood ......................................................................................... 14

Chemical measurements on blood ..................................................................... 14

Statistical methods ......................................................................................................... 16

III. Normal blood factors .................................................................................................... 17

Physical measurements _................................................................................................... 17

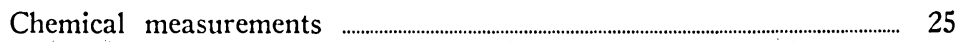

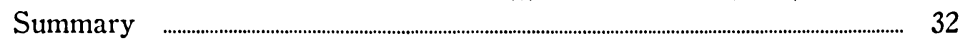

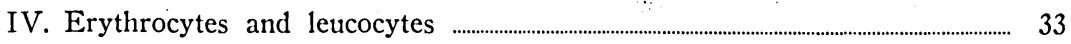

Erythrocyte counts ……................................................................................................ 33

Cell size ......................................................................................................................... 35

Total cell volume .......................................................................................................................... 38

Cell volume per cent ............................................................................................................ 43

Interrelationships between count, size, and volume of cells ....................... 45

Oxygen-combining capacity ................................................................................................. 45

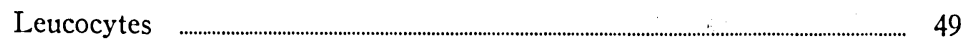

Discussion _.......................................................................................................................................... 51

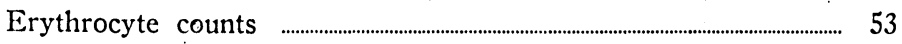

Cell size .............................................................................................................................. 53

Total cell volume ................................................................................................................. 55

Cell volume per cent ....................................................................................... 57

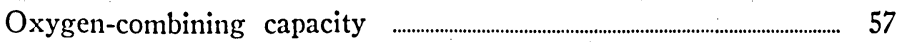

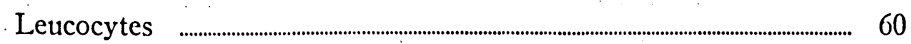

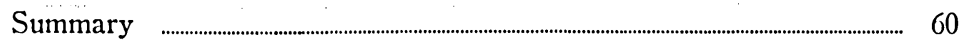

Erythrocyte counts …............................................................................................... 60

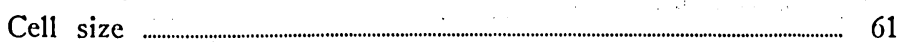

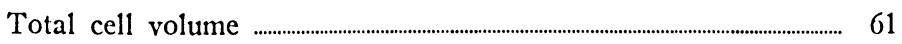

Cell volume per cent ........................................................................................ 62

Interrelationships between red cell count, size, and volume of cells 
Page

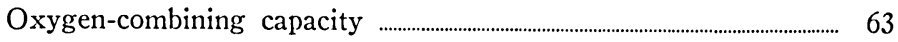

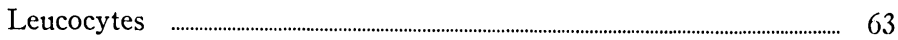

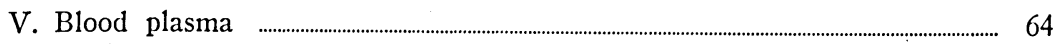

Plasma volume ........................................................................................................................... 64

Blood plasma constituents ............................................................................................... 65

Protein nitrogen ............................................................................................................. 65

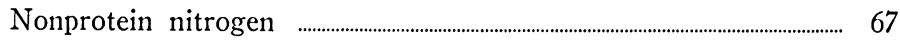

Phosphorus and calcium fractions ..................................................................... 67

Plasma protein fractions: albumin ........................................................................ 71

Globulin ........................................................................................................................... 71

Albumin-globulin ratio ................................................................................... 71

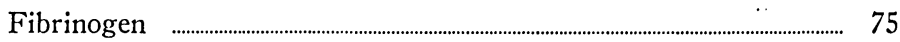

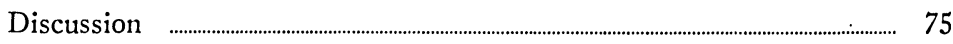

Plasma volume ....................................................................................................................... $\quad 75$

Blood plasma constituents ................................................................................. $\quad 75$

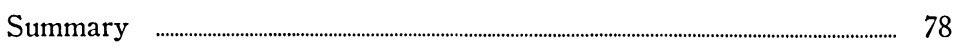

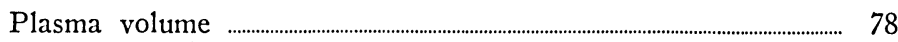

Blood plasma constituents ........................................................................................ $\quad 78$

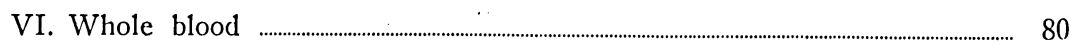

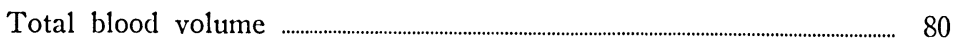

Constituents of whole blood ..................................................................................................... 81

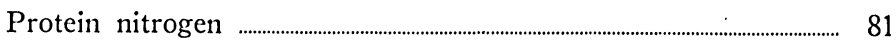

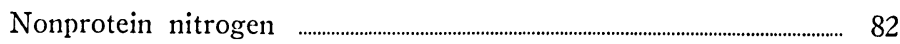

Urea nitrogen ...................................................................................................................... 83

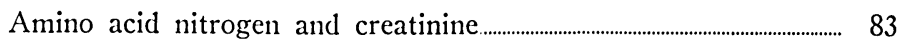

Discussion ................................................................................................................................... 85

Total blood volume ................................................................................................ 85

Constituents of whole blood ............................................................................... 85

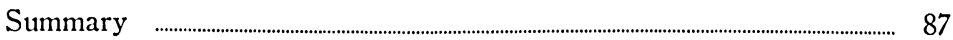

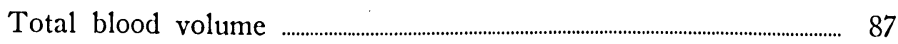

Constituents of whole blood .......................................................................................... 87

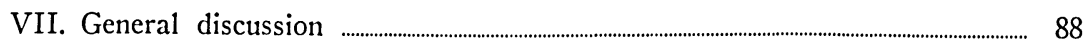

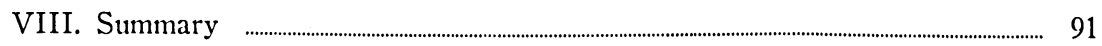

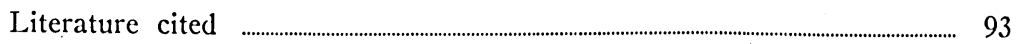

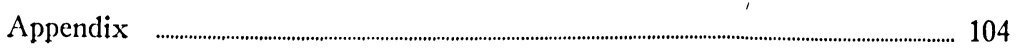


1. Sizes of hemorrhages reported in the literature

2. Physical measurements on the blood of 50 normal dogs ( 16 females, 34 males)

3. Physical measurements on the blood of normal dogs cited in the literature $20-21$

4. Differential white cell counts on the blood of normal dogs cited in the literature

5. Differential white cell counts on the blood of normal dogs (5 males) ..........

6. Chemical measurements on the blood of normal dogs (13 females, 33 males)

7. Chemical measurements on plasma of normal dogs (7 males)

8. Chemical measurements on the blood of normal dogs cited in the literature

9. Chemical measurements on plasma of normal dogs cited in the literature (nitrogenous constituents)

10. Chemical measurements on plasma of normal dogs cited in the literature (phosphorus and calcium fractions)

11. Weights of experimental dogs, in kilograms

12. Values for red cell counts, in millions per cubic millimeter (short-term study)

13. Values for red cell counts, in millions per cubic millimeter (long-term study)

14. Values for mean corpuscular volume, in cubic microns (short-term study)

15. Values for red cell diameters, in microns (long-term study)

16. Values for mean corpuscular volume, in cubic microns (long-term study)

17. Effect of repeated hemorrhage on values for mean corpuscular volume, in cubic microns, and red cell diameter, in microns

18. Actual and predicted total cell volume, in cubic centimeters, 30 minutes and 24 hours after bleeding (short-term study)

19. Values for cell volume, in cubic centimeters per kilogram (short-term study)

20. Values for cell volume, in cubic centimeters per kilogram (long-term study)

21. Amount of synthetic ration consumed, in grams per kilogram (six-weck period) (long-term study)

22. Percentage of total cell volume removed through bleeding and weekly sampling (six-week period) (long-term study)

23. Cell volume regenerated, in cubic centimeters per kilogram (six-week period) (long-term study)

24. Cell volume regeneration in cubic centimeters per kilogram per week (long-term study) 
25. Values for cell volume per cent (short-term study)

Page

26. Values for cell volume per cent (long-term study).

27. Values for oxygen-combining capacity, in volumes per cent (short-term study)

28. Values for oxygen-combining capacity, in volumes per cent (long-term study)

29. Oxygen-combining capacity-comparison of normal levels with levels observed six weeks after hemorrhage (long-term study)

30. Hemoglobin regeneration in grams per kilogram per week (long-term study)

31. Values for leucocyte counts, in thousands per cubic millimeter (shortterm study)

32. Values for leucocyte counts, in thousands per cubic millimeter (longterm study)

33. Values for leucocyte counts, in thousands per cubic millimeter (dogs subjected to repeated bleedings)

34. Differential leucocyte counts (dogs subjected to repeated bleedings)...........

35. Values for plasma volume, in cubic centimeters per kilogram (short-term study)

36. Values for plasma volume, in cubic centimeters per kilogram (long-term study)

37. Values for protein nitrogen in plasma, in grams per 100 cubic centimeters (short-term study)

38. Comparison of the mean total amounts of each of the plasma constituents in the circulation before and after hemorrhage (short-term study)

39. Values for nonprotein nitrogen in plasma, in milligrams per 100 cubic centimeters (short-term study)

40. Values for lipide phosphorus in plasma, in milligrams per 100 cubic centimeters (short-term study)

41. Values for inorganic phosphorus in plasma, in milligrams per 100 cubic centimeters (short-term study)

42. Values for total phosphorus in plasma, in milligrams per 100 cubic centimeters (short-term study)

43. Values for diffusible calcium in plasma, in milligrams per 100 cubic centimeters (short-term study)

44. Values for nondiffusible calcium in plasma, in milligrams per 100 cubic centimeters (short-term study)

45. Albumin in plasma, in grams per 100 cubic centimeters.

46. Globulin in plasma, in grams per 100 cubic centimeters................................................

47. Albumin-globulin ratio in plasma

48. Osmotic pressure of plasma proteins, in millimeters of mercury (calculated on basis of Govaerts' factors) 
49. Fibrinogen in plasma, in grams per 100 cubic centimeters ................................... $\quad 74$

50. Values for total blood volume, in cubic centimeters per kilogram (shortterm study)

51. Values for total blood volume, in cubic centimeters per kilogram (longterm study) meters (long-term study) centimeters (long-term study) meters (long-term study)

\section{APPENDIX TABLES}

I. Physical and chemical measurements on dog No. 44 (mean weight, 22.9 kilograms)

II. Physical and chemical measurements on dog No. 45 (mean weight, 18.6 kilograms)

III. Physical and chemical measurements on dog No. 46 (mean weight, 17.7 kilograms)

IV. Physical and chemical measurements on dog No. 47 (mean weight, 18.9 kilograms)

V. Physical and chemical measurements on dog No. 20 (mean weight, 19.3 kilograms)

VI. Physical and chemical measurements on dog No. 21 (mean weight, 11.7 kilograms)

VII. Physical and chemical measurements on dog No. 22 (mean weight, 12.9 kilograms)

VIII. Physical and chemical measurements on dog No. 19 (mean weight, 18.0 kilograms)

IX. Physical and chemical measurements on dog No. 32 (mean weight, 17.6 kilograms)

$\mathrm{X}$. Physical and chemical measurements on dog No. 17 (mean weight, 16.8 kilograms)

XI. Physical and chemical measurements on dog No. 18 (mean weight, 20.8 kilograms)

XII. Physical and chemical measurements on dog No. 30 (mean weight, 17.0 kilograms)

XIII. Physical and chemical measurements on dog No. 31 (mean weight, 21.1 kilograms) 


\section{ABSTRACT}

This bulletin covers a comprehensive study of blood changes occurring in hemorrhagic anemia induced in dogs maintained on a synthetic diet. A comparison is drawn between the blood picture observed in this experimental anemia and that noted in idiopathic hypochromic anemia.

Many data, consisting of physical and chemical measurements on the blood of normal dogs, are presented. These data, used in selecting normal animals for the experiments, represent the goals of achievement in evaluating nutritional programs designed to restore lost blood.

The following factors return to normal relatively rapidly after bleeding: plasma volume, total calcium and phosphorus in the circulating plasma, serum albumin, and fibrinogen.

Factors that return to normal slowly or remain persistently below normal modify the blood picture and become the measurements used in assessing any corrective nutritional regimé. These include individual red cell volume and diameter, total blood volume, total cell volume, cell volume per cent, hemoglobin, serum globulin, and protein nitrogen of whole blood.

Constituents for which no marked trends were observed are leucocytes, nonprotein nitrogen, urea, creatinine, and amino acid nitrogen.

Statistically established interrelationships among certain of the variables are discussed.

The implications of the similarities between idiopathic hypochromic anemia and hemorrhagic anemia are considered. The anemia induced by hemorrhages, uncomplicated by nutritional deficiencies, pregnancy, lactation, menstruation, or infections, is of the hypochromic microcytic type. 


\section{The Blood Picture in Hemorrhagic Anemia}

\section{INTRODUCTION}

$\mathrm{T}$

The TERM ANEMIA covers a number of abnormal conditions of the blood in which changes are found in the hemoglobin content, or the number, size, and composition of erythrocytes.

The causes of anemia are as varied as the blood pictures. In nutritional anemia there is a deficiency of substances essential to the synthesis of hemoglobin, such as iron or copper. In pernicious anemia there is a lack of compounds needed for building the stroma of the red blood cells. In hemorrhagic anemia there are abnormalities which arise from blood losses. In the latter an individual's condition may be seriously affected by the amount of blood lost in a single hemorrhage, by the total amount of blood lost in a series of small hemorrhages, or through continuous slow bleeding.

Hemorrhages occur in the alimentary or genito-urinary tracts, in accidents, operations, childbirth, or menstruation. In lesions in the alimentary tract or in the genito-urinary tract, blood is commonly mixed with secretions or other materials which make accurate measurement difficult. Ordinarily, accidents take place under conditions which preclude measurement of the blood lost. Data compiled in table 1 illustrate blood losses occurring in operations, childbirth, and menstruation, as cited in the literature.

The seriousness of the hemorrhages recorded in table 1 may be appreciated, in part, by comparing the means and ranges of blood losses with available data on total blood volumes in men and women. Rowntree, Brown, and Roth (1929) reported a mean blood volume of 6,040 cc. $($ S.D. $=758$ cc.) for 49 healthy men, and 5,076 cc. (S.D. $=480$ cc.) for 25 normal women. The loss of a few cubic centimeters of blood will, therefore, constitute a very small percentage of the total volume, whereas a liter will be equivalent to a fifth or a sixth of an adult's total blood volume.

The significance of the hemorrhages shown in table 1 depends upon the rate at which bleeding occurs, upon the relative amount or percentage of total blood volume involved, upon the ease with which an individual replaces the constituents of the blood, and upon the interference, caused by henorrhage, with normal physiological functions of the body. A large hemorrhage may be serious because of the relatively great quantity of 
blood lost in a brief period and an individual's inability to adjust himself to the sudden change. Small repeated losses may become grave because of their cumulative effect and failure of the organism to recover from one hemorrhagic loss before another hemorrhage occurs. Thus Wright

Table 1. Sizes of Hemorrhages Reported in the Literature

\begin{tabular}{|c|c|c|c|c|c|}
\hline \multirow[t]{2}{*}{$\begin{array}{l}\text { Nature of } \\
\text { hemorrhage }\end{array}$} & \multirow[t]{2}{*}{$\begin{array}{l}\text { No. } \\
\text { of } \\
\text { cases }\end{array}$} & \multirow[t]{2}{*}{$\begin{array}{l}\text { Mean } \\
\text { loss } \\
\text { in cc. }\end{array}$} & \multicolumn{2}{|c|}{$\begin{array}{l}\text { Range of } \\
\text { losses } \\
\text { in cc. }\end{array}$} & \multirow[t]{2}{*}{ Investigator } \\
\hline & & & & & \\
\hline Miscellaneous & 35 & 172 & 4 to & 816 & Gatch \& Little (1924) \\
\hline Miscellaneous & 18 & 195 & 8 to & 1,272 & Coller \& Maddock (1932).. \\
\hline Miscellaneous & 74 & $459^{*}$ & 10 to & $1,900 *$ & Buddington \& Taylor (1938) \\
\hline 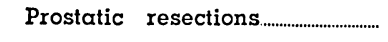 & 55 & 479 & & & Pilcher \& Sheard (1937) \\
\hline $\begin{array}{l}\text { Prostatic resections, with } \\
\text { improved technique............................. }\end{array}$ & 55 & 291 & & & Pilcher \& Sheard (1937) \\
\hline 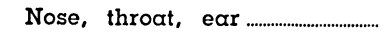 & 924 & 165 & 0 to & 887 & McKenty (1937) \\
\hline Postpartum & 1,870 & 271 & & & Pastore $(1937) \dagger$ \\
\hline Menstrual & 50 & 37 & 9 to & 207 & $\begin{array}{l}\text { Barer, Fowler, and } \\
\text { Baldridge (1934-35) }\end{array}$ \\
\hline 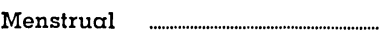 & 100 & 51 & 7 to & 179 & Barer \& Fowler (1936) \\
\hline 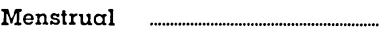 & 4 & 33 & 24 to & 52 & Leverton \& Roberts (1937) \\
\hline
\end{tabular}

* Estimated from graphs.

† 133 patients, loss of 600 cc. or more. Maximum of 1,650 cc. in 2,370 cases.

(1933), Barer, Fowler, and Baldridge (1934-35), and Barer and Fowler (1936) postulated that in some women who suffer from microcytic or hypochromic anemia of undetermined origin, the iron or hemoglobin lost during menstruation is not replaced entirely before subsequent menstrual periods, with the result that a persistent anemia develops. Hypochromic anemia is found infrequently in men, and Witts (1931) regards its occurrence as evidence of blood loss.

The primary objective of this investigation was to secure a comprehensive picture of blood changes occurring in hemorrhagic anemia. Using normal adult dogs as experimental animals, bleedings were induced to simulate in size and number hemorrhages that occur in human subjects under such conditions as those following accidents, childbirth, excessive menstrual losses, and the like.

A further objective was to point out the factors constituting the most accurate gauge of the status or condition of an individual experiencing loss of blood, as well as those which most accurately measure progress made toward recovery. Those which return to normal slowly, or remain persistently below normal, become the problems for which solutions are most urgently needed. Any corrective regime to be adjudged wholly successful must accomplish a complete restoration of all of these factors.

The present investigation dealt with changes that occurred in the blood picture of 19 male and female dogs, observed for varying lengths of time following the removal of significant amounts of blood. This 
bulletin presents a comprehensive description of the changes resulting from severe hemorrhages and the progress of recovery in the animals, maintained on what is believed to be an adequate synthetic diet for adult dogs.

Those factors in the blood picture which changed after bleeding were carefully observed in order to ascertain which of them returned to normal rapidly and apparently with ease, and which of them did not return to normal at all or whose return was greatly delayed.

The authors accumulated considerable data comprising physical and chemical measurements on the blood of 50 normal dogs. These were of value for making comparisons between prehemorrhagic and posthemorrhagic blood pictures and for selecting animals suitable for these experiments. In evaluating any nutritional program designed to restore lost blood, the normal blood picture becomes the goal of achievement.

Since the inception of this research, extensive literature relating to idiopathic hypochromic anemia has appeared. A comparison will be drawn between the blood picture observed in this type of anemia and that noted in experimental hemorrhagic anemia, and the implications of the similarities noted will be discussed.

\section{METHODS}

\section{SELECTION AND CARE OF EXPERIMENTAL ANIMALS}

$\mathrm{N}$

ORMAL ADULT DOGS were considered most acceptable for the purposes

of these experiments, since it has been found that they can be maintained satisfactorily for many months on a synthetic diet. Various foods can be substituted for a part of the synthetic ration and can be tested. The blood volume of dogs can be determined with relative ease, and aliquots of the previously measured blood volume can be removed to induce hemorrhages of desired severity. Furthermore, working with dogs, it is possible to remove sufficiently large samples of blood to permit numerous simultaneous physical and chemical measurements.

Although hemorrhagic anemia occurs frequently in human subjects as a result of blood losses, often the size of a hemorrhage cannot readily be determined. Sometimes investigators find complicating conditions of pathological or physiological nature. Moreover, human subjects are not available for experimental procedures in which measured amounts of blood must be withdrawn, the diet restricted and controlled over considerable periods of time, and significant amounts of blood removed at frequent intervals for determining blood volumes and securing various other physical and chemical measurements.

Every effort was made to select adult dogs in good physical condition and of sizes suitable for the hemorrhages and blood sampling an- 
ticipated. During a preliminary laboratory period, the dogs' reaction to the synthetic diet and their fitness for blood sampling were carefully observed. The animals were kept in individual cages, in a wellventilated, sunny laboratory. Care was taken to maintain a comfortable temperature, and to keep the dogs, the laboratory, and all equipment scrupulously clean.

\section{SYNTHETIC DIET}

The synthetic diet used was a modification of that proposed by Karr (1920) and consisted of:

\begin{tabular}{|c|c|c|}
\hline & Part & ts 1 \\
\hline Casein & 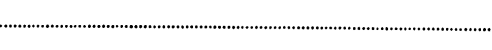 & \\
\hline $\begin{array}{l}\text { Sucrose } \\
\text { Cod-liver }\end{array}$ & 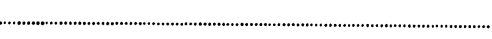 & \\
\hline $\begin{array}{l}\text { Cod-liver oil } \\
\text { Lard ................... }\end{array}$ & $\ldots$ & \\
\hline Lard ................... & & \\
\hline Bone ash ........... & 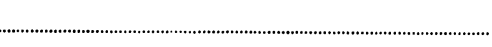 & \\
\hline Salt mixture ... & 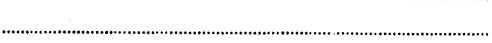 & \\
\hline Dried yeast ..... & & \\
\hline
\end{tabular}

The formula for the salt mixture was as follows:

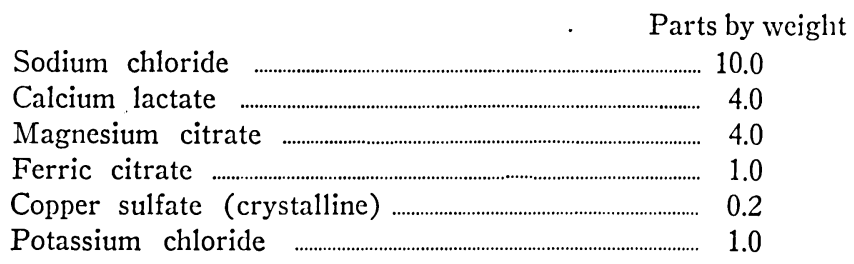

The dogs were given sufficient food to maintain a weight equilibrium. The adequacy of this ration was shown by the fact that three dogs, numbers 57, 58, and 59 (table 2), kept on the diet exclusively for 20 months except for those periods when test foods were added, remained in excellent physical condition throughout the experiment. Furthermore, four dogs, numbers $36,37,38$, and 39 given the same diet exclusively for six to eight months, were also adjudged perfectly healthy at the end of the investigation. None of the commonly accepted evidences of vitamin deficiency were noted at any time.

\section{EXPERIMENTAL PROCEDURE .}

Four groups of animals were used for this investigation. In all cases reported, anemia was induced through the removal, by venipuncture, of a measured amount of blood from the jugular vein.

The first group comprised four male dogs, numbers 44, 45, 46, and 47, maintained at a reasonably constant weight on the synthetic diet already described. For convenient reference, this series of experiments will be designated as the "short-term study." That each dog might 
serve as his own control, normal levels for all the factors studied were set by at least four determinations before each unit hemorrhage had removed approximately 20 per cent of the previously established total blood volume. Samples were taken for subsequent measurement, first, within 30 minutes after the start of bleeding, and thereafter on each of the five or six days immediately following. To portray prebleeding and postbleeding levels adequately, it was necessary to remove from each animal daily 65 to $70 \mathrm{cc}$., representing approximately five per cent of the total blood volume. While these are in fact appreciable blood losses, they are nevertheless comparable to similar losses often experienced before or after severe hemorrhages. In adult human subjects a unit hemorrhage of this magnitude would correspond to the loss of one quart of blood in a single hemorrhage, followed by smaller losses during the week following. Three of the dogs in this group were subjected to two large bleedings; the fourth was bled four times at monthly intervals. After hemorrhage, each animal was allowed a recovery period of not less than four weeks, and during the first two weeks of recuperation 100 grams of beef liver daily replaced a part of the synthetic ration.

The second group consisted of nine animals, five females, numbers $20,21,22,19$, and 32 , and four males, numbers $17,18,30$, and 31 . The dogs were maintained on the same synthetic diet as that used for the short-term study. Normal levels were established by two or more determinations, following which the animals were rendered anemic by two large bleedings spaced two or three days apart, in each of which one fourth of the predetermined total blood volume was removed. These two large bleedings represent as severe hemorrhages as human subjects can experience without fatality. Weekly determinations were made for a long period, 12 weeks in most cases, during which the progress of recovery was carefully observed. For convenient reference, this second series of experiments will be designated as the "long-term study".

In a third study, three additional male dogs, numbers 36,37 , and 38 , were maintained on the same synthetic ration for a period of approximately six months. Normal levels were established as before, after which these animals were subjected to repeated bleedings (four or five). Numerous data were accumulated covering these animals which will be reported in a later publication. In this bulletin, however, only that portion of the data acquired which dealt with changes in cell size and total and differential white cell counts will be discussed.

A fourth group of dogs, consisting of three males, numbers 54, 55, and 56 , were subjected to repeated bleedings (three or four), following the establishment of normal levels. These dogs were also maintained on the synthetic ration used for the other studies and were observed for a period of three to three and one-half months. Again considerable data were accumulated, but reference in this bulletin is confined to that portion pertaining to plasma protein fractions. 
These four studies are thought to be representative of various combinations of large and small hemorrhages which occur in human subjects, with respect to quantities of blood lost and the rate of bleeding. It is anticipated that the data obtained will give a complete picture of the changes occurring in the blood following hemorrhage and will throw light on some of the difficulties inherent in complete recovery.

\section{PHYSICAL MEASUREMENTS ON BLOOD}

Blood volume was measured, using Hooper, Smith, Belt, and Whipple's (1920) modification of Keith, Rowntree, and Geraghty's (1915) dye method. To make fair comparisons among dogs of different sizes, both blood volume and red-cell volume were expressed in terms of cubic centimeters per kilogram of body weight. Cell volume per cent was also included in the data.

To secure red-cell counts and cell-diameter determinations, two separate dilutions of blood were made in Hayem's solution and shaken for three minutes. Then a drop of each was spread on a Levy-Hauser counting chamber. The cells lying in 100 consecutive squares were counted, after which measurements of the red cells were made on the same slide, using a number 1-A cover slip to insure greater accuracy in measuring. Measurements of one diameter of 50 red blood cells were made for dogs 17 to 22,30 to 32 , and 36 and 37 , by means of a calibrated Bausch and Lomb filar micrometer, using a magnification of 560 diameters. Readings were made to 0.2 micron. Since only one diameter was measured, it was necessary to select only round cells for measurement, which introduced a certain degree of selectivity into the measurements and therefore also a proportionate degree of inaccuracy. To determine the extent of error in sampling owing to measuring only 50 cells, duplicate sets of 50 cells each were measured on the same slide in a series of 10 consecutive slides. The maximum difference obtained was 0.14 micron, and the mean difference was 0.07 micron.

\section{CHEMICAL MEASUREMENTS ON BLOOD}

For determining the oxygen-combining capacity of the blood, the method described by Van Slyke (1917) and modified by Van Slyke and Neill (1924) was employed. For total and nonprotein nitrogen, the micro Kjeldahl method of Folin and Wu (1919), and the apparatus recommended by Parnas and Wagner (1921), with the modification suggested by Pregl (1924), were employed. In the short-term study, blood plasma was used for these determinations, whereas in the longterm study whole blood was used.

Determinations on the blood filtrate, prepared according to Folin's (1922) method for laked blood, included measurements of the nonprotein nitrogen by the micro Kjeldahl method; urea nitrogen, by Beattie's (1928) colorimetric method; preformed creatinine, by the 
colorimetric method of Folin and Wu (1919) ; and amino acid nitrogen, by Van Slyke's (1913-14) gasometric method. The protein nitrogen of whole blood, as well as of blood plasma, was determined by the difference between the values for total nitrogen and nonprotein nitrogen.

For analyzing calcium and phosphorus, heparinized plasma was used. Although Holt (1931-32) reported appreciable amounts of calcium in heparin samples, and Kay and Byrom (1927) reported that heparin preparations contained organic phosphorus, the results of the present investigation did not confirm these observations. The addition of heparin to suitable inorganic salt solutions, in an amount equivalent to that used as the anticoagulant, did not influence the results obtained for either element.

Plasma proteins were precipitated by trichloracetic acid according to the method of Greenwald (1915, 1918). The oxidation of organic matter was carried out in an oil bath at $180^{\circ}$ to $200^{\circ} \mathrm{C}$., preliminary to the measurement of total phosphorus. The measurement of inorganic phosphorus was carried out on a.trichloracetic acid filtrate, and the lipide phosphorus was extracted from plasma by the procedure of Bloor (1918). The colorimetric method of King (1932) was used for the phosphorus fractions. Both total and diffusible calcium were determined by the Roe and Kahn (1929) modification of Briggs' (1924) colorimetric method. The sample used for measuring diffusible calcium was obtained by the ultrafiltration of plasma for three hours through a collodion membrane at a pressure of 150 to $180 \mathrm{~mm}$. of mercury. The nondiffusible calcium was calculated by difference after applying a correction for the volume occupied by plasma proteins (Smith and Sternberger, 1932).

For the determination of the plasma protein fractions, the fibrinogen was separated from the plasma, using the method of $\mathrm{Wu}$ (1922). A portion of this filtrate was used for the determination of the combined albumin and globulin content. The albumin fraction was determined on the filtrate after precipitating the globulin with sodium sulfate, using the method described by Howe $(1921 \mathrm{a}, \mathrm{b})$. The globulins were determined by difference.

The filtrate for the determination of nonprotein nitrogen was prepared according to the method of Folin and $\mathrm{Wu}$ (1919).

Duplicate samples of all filtrates were digested, using Folin and Svedberg's (1930) digestion mixture, and the nitrogen content determined by the nesslerization technique of Folin (Folin and Denis, 1916; Folin and Wu, 1919). The Nessler's reagent employed was prepared according to the method of Koch and McMeekin (1924). After nesslerization the samples were read colorimetrically against the standard prepared from ammonium sulfate.

Mean corpuscular volume, in cubic microns, was calculated using the formula of Wintrobe (1931). 


\section{STATISTICAL METHODS}

The data presented have been calculated and checked according to the formulas cited below.

To establish the degree of interdependence between various factors, correlation coefficients were calculated according to the formula:

$$
r_{x y}=\frac{\Sigma(x y) / N-\bar{x} \bar{y}}{S_{x} S_{y}}
$$

As a criterion of significance in the interpretation of the data, the following formula was used:

$$
\mathrm{t}=\frac{\mathrm{r} \sqrt{\mathrm{n}}}{\sqrt{1-\mathrm{r}^{2}}} \quad(\mathrm{n}=\mathrm{N}-2)
$$

and the probability of " $\mathrm{t}$ " being exceeded solely through errors of random sampling was found in a suitable table constructed by Fisher (1928). In most instances, the 0.05 level of significance was considered satisfactory.

In a number of cases, it was desired to test the significance of the difference between two means. Where there was no possibility of a correlation existing between the two variables whose mean difference was being studied, Student's "t" test (Fisher, 1928) was employed, using the formula:

$$
t=\frac{(\vec{x} \bar{y}) \sqrt{n}}{\sqrt{N_{x} S_{x}^{2}+N_{y} S_{y}^{2}}} \sqrt{\frac{N_{x} N_{y}}{N_{x}+N_{y}}}
$$

where $\mathrm{n}=\mathrm{N}_{\mathrm{x}}+\mathrm{N}_{\mathrm{y}}-2$

with a final check upon the probability of " $t$ " being exceeded solely through errors of random sampling.

Where the two variables were paired, thus introducing the possibility of a correlation existing between them, the standard error of the mean difference was determined from the formula:

$$
\mathrm{SE}_{\overline{\mathrm{d}}}=\frac{\mathrm{S}_{\mathrm{d}}}{\sqrt{\mathrm{N}-1}}
$$

the significance of this standard error being determined as described above after calculating:

$$
\mathrm{t}=\frac{\overline{\mathrm{d}}-0}{\mathrm{SE}_{\mathrm{d}}}
$$




\section{NORMAL BLOOD FACTORS}

I $\mathrm{N}$ EXPERIMENTs that involve a study of changes occurring in the 1 blood, the investigator must have available information relative to the normal range of the various blood constituents. Only a few reports included in the literature cited contained reasonably complete information on large groups of animals. The data acquired in the present investigation include chemical and physical measurements on the blood of 50 experimental dogs (16 females, 34 males).

\section{PHYSICAL MEASUREMENTS}

In table 2 are presented data covering both male and female dogs. The values reported for individual animals are in most cases the means of two or more duplicate or triplicate determinations. Based on body weight, the mean total blood volume for these animals was $76.4 \mathrm{cc}$. per kilogram (70.9 cc. for 16 females and $79.0 \mathrm{cc}$. for 34 males). Comparison of the females' range with the males' reveals no significant sex difference. The total blood volume for the females, for example, ranged from 59.0 to $96.5 \mathrm{cc}$. per kilogram of body weight, as against 60.3 to $104.1 \mathrm{cc}$. per kilogram for the males. Similarly, cell volume per cent ranged from 37.7 to 59.0 per cent for the females as compared with 37.6 to 59.3 per cent for the males. Within both groups, however, individual differences were relatively large. Wide variations were apparent in red cell counts in millions per cubic millimeter of blood, ranging from 6.24 . to 8.36 millions for the females, and from 5.08 to 8.55 millions for the males. These individual differences, therefore, emphasize the importance of securing normal values for each animal used in an investigation involving close observation of a changing blood picture.

Blood volume is frequently reported in terms of percentage of body weight. According to Peters and Van Slyke (1931), "The volume of the circulating blood of normal human beings and animals has been variously estimated by different observers using diverse methods, as from one-tenth to one-twentieth of the body weight." In the present study, the mean total blood volume of the 50 experimental animals, 76.4 cc per kilogram, was converted from volume of blood per kilogram to weight of blood per kilogram by using the specific gravity of blood (1.056) reported by Sherrington and Copeman (1893), producing a value of $72.3 \mathrm{~g}$. per kilogram. In other words, blood constituted 7.23 per cent of the body weight of these experimental animals.

Table 3 covers data cited in the literature on normal dogs. A comparison drawn between these values and the results of the present investigation showed that the mean total blood volume observed for the dogs used in this study was lower than that reported by others who had 
used the same method. Hooper, Smith, Belt, and Whipple (1920) using vital red as their dye, reported a mean total blood volume, for a group of 22 animals, of $101.3 \mathrm{cc}$. per kilogram of body weight. Camero and Krumbhaar (1933) reported a mean of 84.8 cc. per kilogram of body weight, while Harris (1920) obtained a mean of $84.1 \mathrm{cc}$. per kilogram using vital red dye, and $71.4 \mathrm{cc}$. per kilogram using Congo red. Powers, Bowie, and Howard (1930), using Congo red as their dye, reported a much higher figure, 112.8 cc. per kilogram, for a group of 25 dogs. The authors' results, using the dye method, coincide more closely with values reported by other investigators who used the carbon-monoxide method and do not agree with the high values reported by some workers using the dye method. Arnold, Carrier, Smith, and Whipple (1921), employing the carbon-monoxide method, reported a mean of 77.8 cc. per kilogram, while Plesch (1909) reported a mean of 79.5 cc., and Gréhant and Quinquad (1882) reported a mean of $81.6 \mathrm{cc}$. per kilogram. Investigators using the direct or Welcker method (Brodin, Richet, and Saint Girons, 1919-20, and Harris, 1920) apparently obtained the lowest values. The hemoglobin injection method produced values of $68.1 \mathrm{cc}$. per kilogram according to Harris (1920), 89.2 cc. per kilogram

Table 2. Physical Measurements on the Blood of 50 Normal Dogs (16 Females, 34 Males)

\begin{tabular}{|c|c|c|c|c|c|c|c|c|}
\hline $\begin{array}{l}\text { Dog } \\
\text { No. }\end{array}$ & $\begin{array}{c}\text { Weight } \\
\text { in } \\
\mathrm{kg} .\end{array}$ & $\begin{array}{c}\text { Total } \\
\text { blood } \\
\text { volume, } \\
\text { in cc. } \\
\text { per } \\
\text { kg. }\end{array}$ & $\begin{array}{l}\text { Cell } \\
\text { vol- } \\
\text { ume, } \\
\text { in cc. } \\
\text { per } \\
\mathrm{kg} .\end{array}$ & $\begin{array}{l}\text { Cell } \\
\text { vol- } \\
\text { ume } \\
\text { per } \\
\text { cent }\end{array}$ & $\begin{array}{c}\text { Red } \\
\text { cell } \\
\text { count, } \\
\text { in } \\
\text { millions }\end{array}$ & $\begin{array}{l}\text { Red } \\
\text { cell } \\
\text { diam- } \\
\text { eter, } \\
\text { in } \\
\text { microns }\end{array}$ & $\begin{array}{l}\text { Mean } \\
\text { corpus. } \\
\text { volume, } \\
\text { in } \\
\text { cubic } \\
\text { microns }\end{array}$ & $\begin{array}{l}\text { White } \\
\text { cell } \\
\text { count, } \\
\text { in } \\
\text { thou- } \\
\text { sands }\end{array}$ \\
\hline \multicolumn{9}{|c|}{ Females (16) } \\
\hline 1 & 16.3 & 69.4 & 33.3 & 48.1 & 7.72 & $\ldots . . . . .$. & 62.3 & 6.04 \\
\hline 2 & 12.0 & 77.9 & 38.2 & 50.0 & 8.07 & ........... & 62.0 & $\ldots \ldots \ldots$ \\
\hline 3 & 13.2 & 66.5 & 31.5 & 47.2 & 7.99 & $\ldots . . . . . .$. & 59.1 & $\ldots \ldots+. .$. \\
\hline 4 & 11.9 & 62.8 & 23.6 & 37.7 & $\ldots \ldots . . .$. & $\ldots \ldots . . .$. & $\ldots . . . \cdot \cdot . \cdot$ & 8.95 \\
\hline 5 & 9.1 & 96.5 & 40.6 & 42.0 & $\ldots . . . \cdots$ & 7.13 & $\ldots \ldots . .$. & $\ldots . . . . .$. \\
\hline 6 & 19.0 & 76.0 & 40.8 & 54.2 & $\ldots \ldots . .$. & $\ldots \ldots \ldots$ & $\ldots \ldots . .$. & $\ldots \ldots \ldots .$. \\
\hline 15 & 14.1 & 68.2 & 30.6 & 44.8 & 7.02 & $\ldots \ldots \ldots . . .$. & 63.8 & 3.85 \\
\hline 16 & 10.0 & 71.9 & 37.6 & 52.6 & 8.36 & $\ldots \ldots . .$. & 62.9 & 5.55 \\
\hline 19 & 17.9 & 71.1 & 42.0 & 59.0 & 7.27 & 6.85 & 81.2 & 4.07 \\
\hline 20 & 17.6 & 71.7 & 29.8 & 41.5 & 6.24 & $\ldots . . . . . .$. & 66.5 & 7.72 \\
\hline 21 & 11.2 & 67.4 & 30.3 & 45.0 & 7.03 & $\ldots \ldots \ldots$ & 64.0 & 9.37 \\
\hline 22 & 12.4 & 59.0 & 26.6 & 45.1 & 6.77 & $\ldots . . . . . .$. & 66.6 & 7.30 \\
\hline 25 & 10.5 & 68.8 & 31.5 & 45.8 & 6.44 & $\ldots . . . . . .$. & 71.1 & 8.70 \\
\hline 26 & 14.7 & 65.8 & 28.8 & 43.7 & 6.66 & $\ldots . . . . .$. & 65.6 & 19.33 \\
\hline 29 & 17.5 & 69.3 & 33.7 & 48.7 & 6.62 & 6.95 & 73.6 & 13.05 \\
\hline 32 & 16.3 & 71.6 & 34.4 & 48.1 & 6.78 & 7.15 & 70.9 & 8.51 \\
\hline Mean & 14.0 & 70.9 & 33.3 & 47.1 & 7.15 & 7.02 & 66.9 & 8.54 \\
\hline Median & 14.1 & 69.4 & 33.3 & 47.2 & 7.02 & 7.13 & 65.6 & 8.51 \\
\hline Range & $9.1-19.0$ & $59.0-96.5$ & 23.6 .42 .0 & $37.7-59.0$ & $6.24-8.36$ & $6.85-7.15$ & $59.1-81.2$ & 3.85-19.33 \\
\hline
\end{tabular}


Table 2-Continued

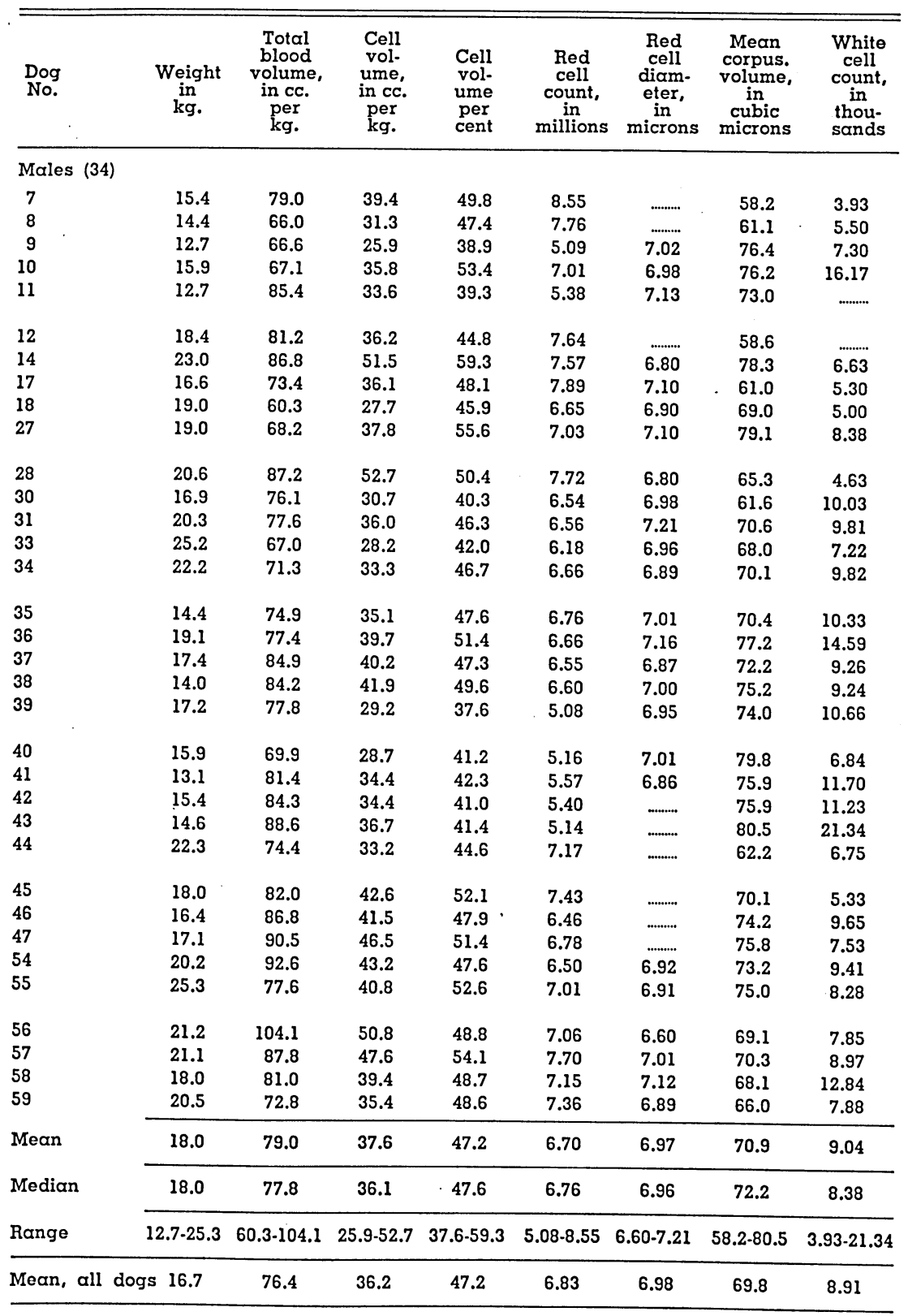

according to Franke and Benedict (1920-21), and $105.3 \mathrm{cc}$. per kilogram according to Lee and Whipple (1921). Attention is directed to the wide range of values obtained by nearly all of the different methods. 
Table 3. Physical Measurements on the

\begin{tabular}{|c|c|c|c|c|c|}
\hline & \multirow{2}{*}{ Reference } & \multirow{2}{*}{$\begin{array}{l}\text { No. } \\
\text { of } \\
\text { dogs }\end{array}$} & \multirow{2}{*}{ Method for blood volume } & \multicolumn{2}{|c|}{$\begin{array}{l}\text { Total blood vol. } \\
\text { in cc. per kg. }\end{array}$} \\
\hline & & & & Range & Mean \\
\hline 1 & This study & $29-50$ & Vital Red & $59.0-104.1$ & 76.4 \\
\hline 2 & Gibson et al. (1938) & 50 & Spectrophotometric Blue Dye & $84.0-97.3$ & $92.7 †$ \\
\hline 3 & Bruner \& Wakerlin (1937) & 34 & & $\ldots$ & $\ldots \ldots \ldots . . .$. \\
\hline 4 & Weech et al. (1937) & 60 & Dye\| & ................................... & $92.9 \mathbb{I}$ \\
\hline 5 & Wintrobe et al. (1935-36) & 54 & & …………….......... & ............... \\
\hline 6 & Ashley \& Guest (1934) & 50 & & .............................. & $\ldots . . . \cdots \cdots$ \\
\hline 7 & Camero \& Krumbhaar (1933) & 6 & Vital Red & 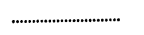 & $84.8 \uparrow$ \\
\hline 8 & Mayerson (1930) & 60 & & …………............ & $\ldots . . . \cdots \cdots$ \\
\hline 9 & Powers et al. (1930) & 25 & Congo Red & $83.0-136.0$ & 112.8 \\
\hline 10 & Kohanawa (1928) & 12 & & ……………........ & $\ldots \ldots \ldots \ldots$ \\
\hline 11 & Pierce \& Plant (1928) & 16 & & ………................... & ............... \\
\hline 12 & Emmons (1927-28) & $\ldots . .$. & & 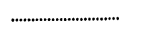 & ............... \\
\hline 13 & Klieneberger (1927) & 6 & & 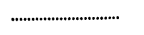 & ............... \\
\hline 14 & Bodansky \& Dressler (1927) & 8 & & $\ldots \cdots \cdots \cdots \cdots \cdots \cdots \cdots \cdots \cdots$ & 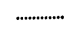 \\
\hline 15 & Bennett (1926) & 7 & & .............................. & $\ldots . \cdots \cdots \cdots \cdot \cdots$ \\
\hline 16 & Gram (1924) & 3 & & 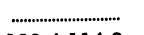 & \\
\hline 17 & Lee \& Whipple (1921) & 9 & Hemoglobin & $100.4-114.2$ & 105.3 \\
\hline 18 & Arnold et al. (1921) & 4 & Carbon Monoxide & 72.1- 89.7 & 77.8 \\
\hline 19 & Whipple \& Robscheit (1921) & 7 & Brilliant Vital Red & $87.0-132.0$ & 109.3† \\
\hline 20 & Franke \& Benedict (1920-21) & 19 & Hemoglobin & $77.2-109.0$ & 89.27 \\
\hline 21 & Harris (1920) & 8 & Direct & $59.0-72.7$ & 65.4 II \\
\hline 22 & Harris (1920) & 6 & Hemoglobin & $60.8-74.6$ & 68.11 \\
\hline 23 & Harris (1920) & 4 & Vital Red & $78.8-88.3$ & 84.1 II \\
\hline 24 & Harris $(1920)$ & 6 & Congo Red & $66.2-76.9$ & $71.4 \mathbb{I}$ \\
\hline 25 & Whipple, Hooper \& Robscheit (1920) & 12 & Vital Red Dry Oxalate & $72.0-146.0$ & 115.07 \\
\hline 26 & Whipple, Hooper \& Robscheit (1920) & 15 & Vital Red Isotonic Oxalate & $89.0-147.0$ & 108.3† \\
\hline 27 & Hooper, Smith et al. (1920) & 22 & Vital Red & $83.7-115.3$ & 101.3 \\
\hline 28 & Brodin et al. $(1919-20)$ & 60 & Direct & ……………........... & 64.47 \\
\hline 29 & Gasser et al. (1919) & $\ldots . .$. & & .............................. & ............... \\
\hline 30 & Kuhl (1919) & 10 & & & ............. \\
\hline 31 & Meek \& Gasser (1918-19) & 8 & Gum Accacia & $78.4-107.8$ & 97.4 \\
\hline 32 & Brodin et al. (1918) & 29 & & $61.0 \cdot 73.0$ & 67.4 \\
\hline 33 & Wells \& Sutton (1915-16) & $\ldots . .$. & & 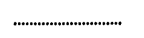 & $\ldots \ldots \ldots \ldots . .$. \\
\hline 34 & Mann (1915) & 6 & Direct (without washout) & 54.0-67.0I & $59.2 \dagger$ \\
\hline 35 & Lamson (1915) & $\ldots . .$. & & 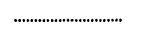 & $\ldots \ldots \ldots \cdots \cdots \cdot \cdots$ \\
\hline 36 & Musser \& Krumbhaar (1914) & 47 & & ................................ & $\ldots . . . \cdots \cdots \cdot \ldots$ \\
\hline 37 & Nassau (1913-14) & 35 & & 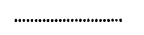 & $\ldots \ldots \ldots \ldots . . . .$. \\
\hline 38 & Musser \& Krumbhaar (1913) & $5 \cdot 17$. & & 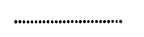 & $\ldots \ldots \ldots \ldots$ \\
\hline 39 & Magnan (1911) & $\ldots . . .{ }^{\circ}$ & & ……………............. & $\ldots . . \cdots \cdots \cdot \cdots$ \\
\hline 40 & Goodall (1910) & $\ldots . .$. & & 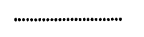 & ................ \\
\hline 41 & Lenze (1909) & $\ldots . .$. & & $\ldots \ldots \ldots \ldots \ldots \ldots \ldots \ldots \ldots \ldots$ & $\ldots \ldots \ldots \ldots . .$. \\
\hline 42 & Nelson (1909) & 2 & Dilution & $67.4-67.4$ & $67.4 I$ \\
\hline 43 & Plesch (1909) & 5 & Carbon Monoxide & 70.6-90.0 & $79.5 \mathbb{I}$ \\
\hline 44 & Burnett \& Traum (1905) & $6-7$ & & {$[. . \cdots \cdots \cdots \cdots \cdots \cdots \cdots \cdots \cdots \cdots$} & $\ldots \ldots \ldots \ldots . .$. \\
\hline 45 & Breuer \& v Seiller (1903) & 3 & & ............................... & ....................... \\
\hline 46 & Busch \& Van Bergen (1902) & 20 & & ............................. & ................ \\
\hline 47 & Biedl \& v Decastello (1901) & $\ldots . .$. & & ................................ & $\ldots . . \cdots \cdots \cdots \cdots$ \\
\hline 48 & Courmont \& Lesieur (1901) & 20 & & ............................... & $\ldots . . . \cdots \cdots \cdot \cdots$ \\
\hline 49 & Dawson $(1900-01)$ & 12 & & ............................... & $\ldots . . . \cdots \cdots \cdot . .$. \\
\hline 50 & Schauman \& Rosenqvist (1898) & 1 & & ............................... & ............... \\
\hline 51 & Sherrington (1894) & $\ldots . . .$. & & ............................... & ............... \\
\hline 52 & Sussdorf (1890) & ........ & & ................................ & ............... \\
\hline 53 & Hayem (1889) & 20 & & $\ldots \ldots \ldots \ldots \ldots \ldots \ldots \ldots \ldots \ldots$ & $\ldots . . . \cdots \cdots \cdots$ \\
\hline 54 & Formad (1888) & $\ldots . .$. & & ................................ & ............... \\
\hline 55 & Ellenberger (1887) & $\ldots . .$. & & ............................. & ................... \\
\hline 56 & Gréhant \& Quinquad (1882) & 9 & Carbon Monoxide & 72.3- 91.6 & $81.6 \mathbb{I}$ \\
\hline 57 & Schmidt (1878) & $\ldots . .$. & & 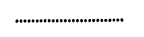 & $\ldots \ldots+\cdots \cdots$ \\
\hline 58 & Manassein (1872) & $\ldots . .$. & & 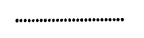 & $\ldots . . . \cdots \cdots \cdot . \cdot$. \\
\hline 59 & Welcker (1863-64) & $\ldots . .$. & & ............................... & $\ldots+\ldots \ldots \ldots$ \\
\hline 60 & Vierordt (1854) & $\ldots . .$. & & ............................... & 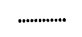 \\
\hline 61 & Schmidt (1848) & $\ldots . .$. & & ………................ & $\ldots . . \cdots \cdots \cdot . .$. \\
\hline
\end{tabular}

* Calculated from total cell volume and weight. †Calculated average. \$Calculated from weight. \| Dye not given. "* Forty-three dogs. 
Blood of Normal Dogs Cited in the Literature

\begin{tabular}{|c|c|c|c|c|c|c|c|c|c|c|}
\hline \multicolumn{2}{|c|}{$\begin{array}{l}\text { Cell volume } \\
\text { in cc. per } \mathrm{kg} \text {. }\end{array}$} & \multicolumn{2}{|c|}{$\begin{array}{l}\text { Cell volume } \\
\text { per cent }\end{array}$} & \multicolumn{2}{|c|}{$\begin{array}{l}\text { Red cell count, } \\
\text { in millions }\end{array}$} & \multirow{2}{*}{$\begin{array}{l}\text { Mean red } \\
\text { cell } \\
\text { diam. in } \\
\text { microns }\end{array}$} & \multirow{2}{*}{$\begin{array}{l}\text { Mean } \\
\text { corp. vol. } \\
\text { in cubic } \\
\text { microns }\end{array}$} & \multicolumn{3}{|c|}{$\begin{array}{l}\text { White cell count, } \\
\text { in thousands }\end{array}$} \\
\hline Range & Mean & Range & Mean & Range & Mean & & & Range & Mean & \\
\hline 23.6-52.7 & 36.2 & $37.6-59.3$ & 47.2 & $5.08-8.55$ & 6.83 & 6.98 & 69.8 & $3.85-21.34$ & 8.91 & 1 \\
\hline $36.4-54.6$ & 43.87 & $38.1-60.0$ & $47.8 \uparrow$ & & & 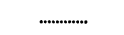 & & $\ldots$ & & 2 \\
\hline$\ldots$ & $\ldots$ & .............................. & 44.3 & $\ldots$ & 6.45 & $\ldots . . . \cdots$ & 68.9 & $\ldots$ & 14.18 & 3 \\
\hline & $44.5^{\star}$ & & 47.5 & $\ldots$ & $\ldots . . . \cdots \cdots$ & $\ldots \ldots \ldots$ & ................ & & $\ldots . . . \cdots \cdots$ & 4 \\
\hline & $\ldots \ldots \ldots \ldots . . .$. & $\ldots$ & 47.3 & ……………….......... & 7.02 & $\ldots \ldots \ldots$ & 67.6 & & $\ldots . . . . . . . . .$. & 5 \\
\hline & $\ldots$ & & 46.9 & 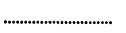 & 6.87 & ..................... & 66.6 & & $\ldots$ & 6 \\
\hline & $40.2 \dagger$ & $25.0-49.0$ & $40.9^{* *}$ & $4.09-8.33$ & $6.95 \dagger$ & ................... & .............. & & $\ldots . . . . . . . .$. & 7 \\
\hline & $\ldots \ldots . . . . . .$. & & 38.6 & 4.86- 8.56 & 6.16 & $\ldots \ldots \ldots$ & 59.3 & $5.65-17.35$ & 11.16 & 8 \\
\hline $39.6-61.3$ & $50.9^{*}$ & $34.4-50.6$ & 45.2 & 4.63- 8.61 & 7.00 & $\ldots . . . . . . . .$. & 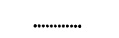 & & & 9 \\
\hline & $\ldots$ & & $\cdots \cdots \cdots \cdot$ & $5.24-7.16$ & 6.21 & 6.80 & $\ldots . . . . . . . .$. & $12.80-19.80$ & 17.10 & 10 \\
\hline & $\ldots . . . . . . .$. & $\ldots \ldots \ldots . . . .$. & $\ldots \ldots \ldots$ & $5.40-6.73$ & $\ldots$ & $\ldots \ldots \ldots \ldots . .$. & 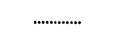 & $8.70-17.90$ & $\ldots$ & 11 \\
\hline & $\ldots \ldots . . . . .$. & & 43.0 & …………............. & 6.25 & 7.20 & 69.0 & & & 12 \\
\hline & ............... & $\ldots$ & $\ldots \ldots \ldots \ldots$ & $6.00-9.76$ & 7.22 & 6.80 & $\ldots$ & $5.70-11.10$ & 10.00 & 13 \\
\hline & ....................... & & $\ldots \ldots \ldots . . . .$. & $7.16-10.50$ & $8.47 \dagger$ & 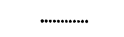 & 50.7 & ………………...... & & 14 \\
\hline & $\ldots \ldots \ldots$ & $31.0-43.0$ & 38.07 & .......................... & 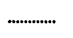 & $\ldots-\ldots \ldots . .$. & 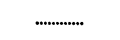 & ........................... & $\ldots$ & 15 \\
\hline & $\ldots . . . . . . .$. & $33.7-52.0$ & $43.7 \dagger$ & 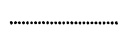 & $\ldots+\cdots \cdots$ & .............. & ............... & ............................... & $\ldots \ldots . . . . . .$. & 16 \\
\hline $36.8-45.7$ & 42.0 & 43.0 & 51.5 & & $\ldots$ & $\ldots . . \cdots \cdots \cdot . .$. & ............... & 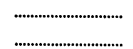 & 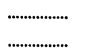 & $\begin{array}{l}17 \\
18\end{array}$ \\
\hline $42.4-73.3^{*}$ & $56.9 \dagger$ & $45.6-59.4$ & $51.7 \dagger$ & $6.90-11.80$ & 8.297 & ........................ & 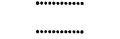 & $7.00-13.40$ & $9.98+$ & 19 \\
\hline & $\ldots \ldots \ldots . . . .$. & $24.0-45.2$ & $36.0 \dagger$ & ………................. & $\ldots \ldots \ldots$ & $\ldots \ldots \ldots$ & $\ldots \ldots . . . . . . .$. & ............................ & .................. & 20 \\
\hline & $\ldots-\ldots . . . . .$. & $\ldots$ & $\ldots \ldots \ldots$ & & $\ldots$ & $\ldots . . . \cdots$ & $\ldots . . . \cdots \cdot . .$. & $\ldots \ldots \ldots \ldots . . .$. & $\ldots$ & 21 \\
\hline & ....................... & $\ldots \ldots \ldots$ & $\ldots$ & $\ldots \ldots \ldots$ & $\ldots . . . \cdots \cdots$ & $\ldots \ldots \ldots \ldots$ & $\ldots . . . \cdots \cdots$ & $\ldots \ldots \ldots \ldots$ & $\ldots \ldots \ldots . . . .$. & 22 \\
\hline & $\ldots . . \cdots \cdots \cdot \ldots$ & 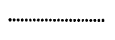 & $\ldots \ldots \ldots \ldots$ & $\ldots \ldots \ldots \ldots$ & 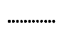 & $\ldots \ldots \ldots \ldots$ & $\ldots \ldots \ldots \ldots . .$. & $\ldots \ldots \ldots \ldots . . .$. & $\ldots$ & 23 \\
\hline $35.7-82.2^{\star}$ & $62.0 \dagger$ & $45.0-60.0$ & 53.17 & $6.60-10.10$ & $7.79+$ & $\ldots \ldots \ldots . . . .$. & ............................ & & & $\begin{array}{l}24 \\
25\end{array}$ \\
\hline $40.3-89.5^{*}$ & $59.9 †$ & $45.5-62.6$ & $55.1 \dagger$ & $6.70-11.40$ & $8.77+$ & ............................ & ……........ & $6.20-14.80$ & $10.52 \dagger$ & 26 \\
\hline $29.1-69.2^{\star}$ & $52.5 \uparrow$ & $34.4-63.5$ & 51.6 & ................................ & $\ldots \ldots \ldots . . . .$. & $\ldots \ldots \ldots . . . .$. & ............... & ................................ & ........................... & 27 \\
\hline & ...................... & & $\ldots$ & & $\ldots$ & $\ldots+\cdots$ & $\ldots . . . . . . .$. & ............................ & $\ldots$ & 28 \\
\hline & $\ldots . . . . . .$. & ....... & 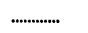 & $5.19-8.17$ & $6.68 \mp$ & 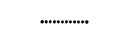 & $\ldots . . . \cdots \cdots$ & .............................. & $\ldots . . . \cdots \cdots \cdots$ & 29 \\
\hline & 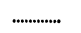 & & $\ldots \ldots \ldots$ & $\ldots$ & $6.59^{\circ}$ & 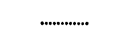 & ....................... & & 12.60 & 30 \\
\hline & $\ldots . . . . . . . .$. & & $\ldots . . . . . . . . .$. & ………………........ & .................... & ............... & .............. & & ................... & 31 \\
\hline$\ldots \ldots \ldots$ & $\ldots . \cdots \cdots \cdots$ & & $\ldots . . . \cdots \cdots \cdot . .$. & $6.00-8.00$ & $6.90 \S$ & $\ldots . . . . . . .$. & $\ldots . . . . . . .$. & $10.00-19.00$ & 14.50 & 32 \\
\hline & $\ldots . . . . . . .$. & $\ldots \ldots . . .$. & $\ldots$ & $\ldots$ & 6.71 & $\ldots . . . . . . . . .$. & .............. & $\ldots$ & 11.00 & 33 \\
\hline & $\ldots . . \cdots \cdots \cdots$ & & 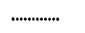 & & .................. & 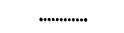 & ....................... & $\ldots$ & 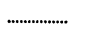 & 34 \\
\hline$\ldots . \cdots \cdots$ & ............... & & $\ldots . . . . . . .$. & $6.74-8.00$ & $7.46 \dagger$ & ....................... & $\ldots . . . . . . . .$. & & 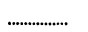 & 35 \\
\hline & $\ldots . \cdots \cdots \cdots . .$. & & $\ldots+. . . \cdots \cdot$ & $4.63-7.76$ & 5.97 & 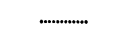 & $\ldots \ldots \ldots$ & $8.80-33.05$ & 15.92 & 36 \\
\hline & $\ldots . . . \cdots \cdots . .$. & & .................. & $5.40-7.30$ & 6.40 & $\ldots . . . \cdots \cdot \ldots$ & $\ldots . . . \cdots \cdots$ & $5.00-13.40$ & 7.70 & 37 \\
\hline & .............. & & $\ldots . . . . . . . .$. & $4.61-5.52$ & $5.20 \dagger$ & $\ldots \ldots . . . .$. & $\ldots+\cdots . . . .$. & $9.00-14.40$ & $12.52 \dagger$ & 38 \\
\hline & .............. & ................... & $\ldots . . . . . . . .$. & $\ldots$ & $\ldots \ldots \ldots$ & 7.00 & $\ldots \ldots . . . . .$. & & & 39 \\
\hline.. & $\ldots . . . \cdots+\cdots . .$. & $\ldots \ldots . .$. & $\ldots$ & 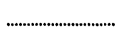 & 5.60 & 7.00 & $\ldots . . . . . . . . .$. & $11.20-31.20$ & 19.50 & 40 \\
\hline & $\ldots . . . . . . . .$. & ........... & $\ldots$ & & $\ldots \ldots \ldots$ & 7.65 & $\ldots \ldots \ldots$ & $\ldots$ & 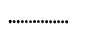 & 41 \\
\hline & $\ldots \ldots \ldots . . . .$. & & .................... & $5.17-5.94$ & $5.56 \uparrow$ & ...................... & 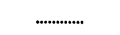 & 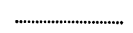 & $\ldots . . . . . . . . . . . .$. & 42 \\
\hline & ............... & & $\ldots \ldots \ldots \ldots . . .$. & 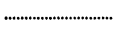 & $\ldots . . . \cdots \cdots \cdots$ & ............... & ................ & & $\ldots$ & 43 \\
\hline & .............. & & ................... & $5.72-6.56$ & $6.03+$ & 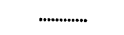 & $\ldots-\ldots . . . .$. & $5.86-8.89$ & $7.60 †$ & 44 \\
\hline & .............. & & $\ldots$ & $6.90-7.95$ & $7.42 \dagger$ & $\ldots . . . \cdots \cdot . .$. & 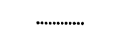 & ……………….......... & ................. & 45 \\
\hline & ............... & $\ldots \ldots . .$. & .................... & $4.23 \cdot 8.03$ & 6.21 & $\ldots . . . \cdots \cdots$ & $\ldots+\cdots, \ldots$ & $7.20-14.38$ & 9.53 & 46 \\
\hline & ............... & $\ldots \ldots \ldots$ & 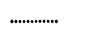 & .......................... & $\ldots \cdots \cdots \cdots \cdots$ & .............. & 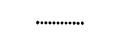 & $\ldots$ & 12.00 & 47 \\
\hline & $\ldots \ldots \ldots \ldots \ldots$ & & .............. & & $\ldots \ldots \ldots \ldots . .$. & .............. & $\ldots \ldots$ & $6.00-10.00$ & .................. & 48 \\
\hline & .............. & & $\ldots . . . . . . . .$. & $6.25 \cdot 8.50$ & 7.21 & 7.00 & $\ldots \ldots \ldots$ & $11.00-28.00$ & 19.30 & 49 \\
\hline & ............... & $\ldots$ & $\ldots$ & $\ldots$ & $\ldots+\cdots \cdots$ & 6.95 & $\ldots \ldots \ldots . . . .$. & $\ldots$ & & 50 \\
\hline & ................ & & $\ldots . . . \cdots$ & $2.50-8.13$ & $5.58+$ & ..................... & 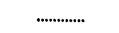 & $6.26-15.90$ & $9.40 †$ & 51 \\
\hline & .................... & & ..................... & ………………..... & 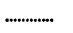 & 7.30 & $\ldots$ & 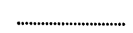 & $\ldots+\cdots \cdots$ & 52 \\
\hline & .............. & & $\ldots . . . . . . . .$. & & 6.65 & 7.20 & 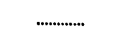 & & $\ldots \ldots \ldots$ & 53 \\
\hline & .............. & & $\ldots . . . . . . .$. & & $\ldots . . . \cdots \cdots$ & 7.00 & & & .................... & 54 \\
\hline &...$\cdots \cdots \cdots$ & & ..................... & & $\ldots \ldots \ldots \ldots \ldots$ & 7.30 & & & $\ldots . . . . . . . . . . . .$. & 55 \\
\hline & 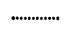 & & ..................... & & 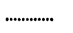 & $\ldots \cdots \cdots \cdots$ & & & $\ldots \ldots . . . . .$. & 56 \\
\hline & 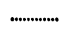 & & 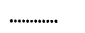 & & & 6.40 & & & $\ldots$ & 57 \\
\hline & $\ldots . . . . . . .$. & & $\ldots \ldots \ldots$ & $\ldots$ & .............. & 6.95 & $\ldots \ldots \ldots$ & & 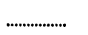 & 58 \\
\hline & 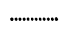 & ...................... & $\ldots \ldots \ldots$ & $\ldots$ & $\ldots \cdots \cdots \cdots$ & 7.30 & $\ldots \ldots \ldots$ & .................... & 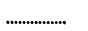 & 59 \\
\hline & 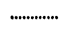 & ....................... & 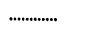 & & .............. & 7.30 & 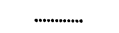 & ............... & 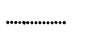 & 60 \\
\hline$\cdots$ & $\ldots \ldots \ldots$ & 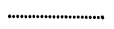 & .............. & ………………...... & ............... & 7.00 & $\ldots \ldots$ & 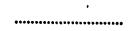 & $\ldots$ & 61 \\
\hline
\end{tabular}


Table 4. Differential White Cells Counts on the

\begin{tabular}{|c|c|c|c|c|c|c|c|c|}
\hline & \multirow{2}{*}{ Reference } & \multirow{2}{*}{$\begin{array}{l}\text { No. } \\
\text { of } \\
\text { dogs }\end{array}$} & \multicolumn{2}{|c|}{$\begin{array}{l}\text { Neutrophiles, } \\
\text { per cent }\end{array}$} & \multicolumn{2}{|c|}{$\begin{array}{c}\text { Small } \\
\text { lymphocytes, } \\
\text { per cent }\end{array}$} & \multicolumn{2}{|c|}{$\begin{array}{c}\text { Large } \\
\text { lymphocytes, } \\
\text { per cent }\end{array}$} \\
\hline & & & Range & Mean & Range & Mean & Range & Mean \\
\hline 1 & This study & 5 & $78.9-87.1$ & 82.6 & $2.2-15.8$ & 9.3 & $1.0 \cdot 4.8$ & 2.5 \\
\hline 2 & Mayerson (1930) & 60 & $56.0-89.0$ & 74.0 & $9.0-34.0$ & 20.0 & $0.0-10.0$ & 4.0 \\
\hline 3 & Pierce \& Plant (1928) & 16 & $69.0-75.0$ & $\ldots . . . \cdots \cdots$ & $19.0-20.0$ & $\ldots . . \cdots \cdots \cdot . .$. & $5.0-7.0$ & ............. \\
\hline 4 & Kohanawa (1928) & 12 & $63.4-84.8$ & 77.4 & 6.6-22.7 & 10.7 & 1.4. 3.9 & 2.5 \\
\hline 5 & Klieneberger (1927) & 6 & $49.0-88.0$ & 77.4 & $21.0-43.6$ & $27.4^{*}$ & $0.5 \cdot 3.4$ & $1.7^{\star}$ \\
\hline 6 & Kuhl (1919) & 10 & ............................. & 57.0 & 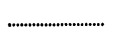 & 25.0 & 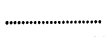 & 8.0 \\
\hline 7 & Burket (1917) & 2 & $43.1-64.0$ & $55.2^{*}$ & 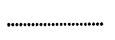 & $\ldots . . \cdots \cdots \cdots \cdot \cdots$ & 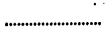 & $\ldots . . \cdots \cdots \cdots \cdots$ \\
\hline 8 & Mann (1916) & 6 & $64.0-79.0$ & 73.0 & $5.0-20.0$ & $11.0^{*}$ & $6.0-13.0$ & $10.0^{*}$ \\
\hline 9 & Musser \& Krumbhaar (1914) & 22 & $40.5-81.0$ & 66.6 & $10.0-51.0$ & 22.1 & $1.0-17.5$ & 6.8 \\
\hline 10 & Nassau (1913-14) & 35 & $63.0-79.0$ & 72.0 & & .............. & (n)................ & ............... \\
\hline 11 & Furno (1913) & 11 & $52.0-84.5$ & $69.2^{*}$ & ............ & ............... & (.......... & .............. \\
\hline 12 & Schittenhelm et al. (1911-12) & 15 & $39.0-82.0$ & 71.8 & .................... & ............... & (.................... & ............... \\
\hline 13 & Goodall (1910) & $\ldots . .$. & $60.0-78.0$ & 63.0 & ............. & .................... & (............... & ............... \\
\hline 14 & Burnett \& Traum (1905) & 7 & $57.9-76.1$ & 68.4 & .............. & ..................... & 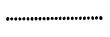 & ............... \\
\hline 15 & Selinoff (1904) & 20 & $69.4-87.2$ & $79.6^{*}$ & 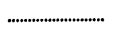 & 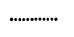 & (.......................... & 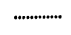 \\
\hline 16 & Reckzeh (1904) & 4 & $70.0-80.0$ & $75.0^{\star}$ & $8.5-16.0$ & $13.9^{\star}$ & $3.0 \cdot 4.0$ & $3.6^{*}$ \\
\hline 17 & Goodall et al. (1903-04) & 1 & 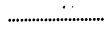 & $60.5 \dagger$ & .......... & ............... & & $\ldots . \cdots \cdots \cdots \cdot \cdots$ \\
\hline 18 & Nicolas \& Dumoulin (1903) & 2 & 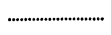 & $72.5^{*}$ & ............. & ............... & & ............... \\
\hline 19 & Busch \& Van Bergen (1902) & 20 & $54.3-87.5$ & 65.7 & & $\ldots \ldots \ldots \ldots$ & & .............. \\
\hline 20 & Biedl \& v Decastello (1901) & ....... & .................................. & 75.0 & & $\ldots . . \cdots \cdots \cdots$ & & 5.8 \\
\hline 21 & Courmont \& Lesieur (1901) & 20 & $52.0-85.0$ & 69.0 & & ................ & & $\cdots \cdots \cdots \cdots$ \\
\hline 22 & Dawson $(1900-01)$ & 12 & $62.4-68.0$ & 64.6 & ............ & ................ & ............. & .............. \\
\hline 23 & Tallqvist \& Willebrand (1899-1900) & 15 & $68.4-83.8$ & $75.1^{*}$ & ................ & ................... & (............. & ............... \\
\hline 24 & Zenoni (1894) & $\ldots . . \cdot$ & ............................. & 62.0 & ............................. & 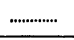 & $\ldots+\cdots \cdots+\cdots \cdots \cdots$ & ............... \\
\hline
\end{tabular}

* Calculated average.

† Calculated from number per cu. mm.

¥ Includes transitional cells.

The mean cell volume per cent noted in the present research, 47.2 per cent, coincides well with the median of the values found in the literature. The wide range of normal values for red cell count reported by various investigators is in agreement with the authors' observation that normal animals may differ markedly in this respect. The mean erythrocyte count noted in the present investigation, 6.83 millions per cubic millimeter, was slightly above the median for the dogs cited in the literature. The mean diameter of red blood cells for the dogs used in this investigation, based on measurements for 30 animals, was 6.98 microns. Burnett (1917), in summarizing the work of six investigators, reported a range of 6.95 to 7.30 microns. Kohanawa (1928) listed a slightly lower figure, 6.80 microns, while Emmons (1927-28) reported a value of 7.20 microns. These differences can probably be explained, at least in part, by the methods used for securing the measurements.

Few workers have included in their reports data on mean corpuscular volume. With the exception of the low value reported by Bodansky and Dressler (1927), based on seven animals, the values included in the 
Blood of Normal Dogs Cited in the Literature

\begin{tabular}{|c|c|c|c|c|c|c|c|}
\hline \multicolumn{2}{|c|}{$\begin{array}{c}\text { Total } \\
\text { lymphocytes, } \\
\text { per cent }\end{array}$} & \multicolumn{2}{|c|}{$\begin{array}{c}\text { Monocytes, } \\
\text { per cent }\end{array}$} & \multicolumn{2}{|c|}{$\begin{array}{c}\text { Eosinophiles, } \\
\text { per cent }\end{array}$} & \multirow{2}{*}{$\begin{array}{l}\text { Basophiles, } \\
\text { per cent }\end{array}$} & \\
\hline Range & Mean & Range & Mean & Range & Mean & & \\
\hline \multirow{2}{*}{$7.0-16.8$} & 11.8 & $0.8-3.8$ & 2.1 & $0.5 \cdot 6.8$ & 2.8 & 0.0 & 1 \\
\hline & 24.0 & $\ldots$ & $\ldots . . . . . . .$. & $0.0-7.0$ & 2.0 & Occasionally & 2 \\
\hline \multirow{2}{*}{$\ldots$} & $\ldots . . . . . .$. & $\ldots$ & $\ldots . . . \cdots \cdots \cdot . .$. & 1.0 .3 .0 & $\ldots \ldots \ldots \ldots . . .$. & $0.0-1.0$ & 3 \\
\hline & 13.2 & 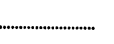 & 6.1 & 1.6- 7.8 & 3.3 & 0.0 & 4 \\
\hline \multirow{2}{*}{ 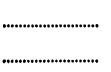 } & $\ldots . . . . . . .$. & ...…………... & $\ldots \ldots \ldots . . . .$. & $3.0-13.0$ & 4.2 & Rare & 5 \\
\hline & 33.0 & 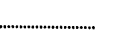 & $\ldots \ldots \ldots \ldots$ & $\ldots$ & 10.0 & 1.0 & 6 \\
\hline \multirow{3}{*}{ 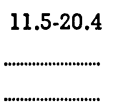 } & $16.9^{*}$ & $0.9-2.4$ & $1.8^{*}$ & $9.3-24.4$ & $18.6^{*}$ & $0.3^{*}$ & 7 \\
\hline & $\therefore . . . \cdots \cdots . .$. & $1.0 \cdot 6.0 \neq$ & $3.5^{*}$ & $0.0-7.0$ & $4.0^{*}$ & $\ldots$ & 8 \\
\hline & $\ldots \cdots \cdots \cdots \cdots$ & $\ldots$ & $\ldots . . . \cdots \cdots$ & $0.0-20.0$ & 5.1 & & 9 \\
\hline \multirow[t]{2}{*}{$13.0-25.5$} & 18.6 & 2.6- $6.5 \ddagger$ & 5.2 & $\ldots$ & $\ldots \ldots \ldots$ & Very seldom & 10 \\
\hline & $\ldots . . \cdots \cdots$ & 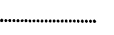 & $\ldots$ & $0.5-2.6$ & $1.5^{*}$ & 4.1 & 11 \\
\hline $12.0-55.0$ & 18.5 & 1.5. $9.5 \ddagger$ & 4.1 & $2.0-11.0$ & 3.7 & & 12 \\
\hline $20.0-35.0$ & 30.0 & $\ldots \ldots \ldots \ldots \ldots \ldots$ & $\ldots . . . . . . .$. & $2.0-14.0$ & 7.0 & & 13 \\
\hline $11.0-28.0$ & 17.6 & $3.1-10.4$ & 6.4 & $3.7-10.5$ & 7.3 & & 14 \\
\hline \multirow{4}{*}{$\begin{array}{r}6.6-16.6 \\
\cdots \cdots \cdots \cdots \\
\cdots\end{array}$} & $12.7^{\star}$ & 2.8- 9.4 & $6.1^{*}$ & $\ldots$ & $\ldots . . \cdots \cdots$ & & 15 \\
\hline & $\ldots \ldots \ldots \ldots$ & $0.0 \cdot 0.5$ & 0.1 & $0.0 \cdot 5.0$ & $2.2^{*}$ & & 16 \\
\hline & $25.0 \dagger$ & 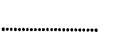 & $\ldots$ & $\ldots$ & $14.5 †$ & & 17 \\
\hline & $10.3^{*}$ & $\ldots . . . . . . . . . . .$. & $17.8^{*}$ & 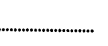 & $3.3^{*}$ & & 18 \\
\hline \multirow[t]{2}{*}{$3.7-35.0$} & 21.0 & $3.7 \cdot 13.2$ & 6.8 & $0.8-15.2$ & 5.3 & & 19 \\
\hline & 15.8 &. & $\ldots \ldots \ldots \ldots$ & 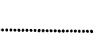 & 3.4 & 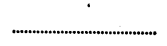 & 20 \\
\hline & $\cdots$ & & $\ldots$ & $\ldots$ & $\ldots \ldots \ldots \ldots$ & & 21 \\
\hline $11.2-31.6$ & 22.2 & $\ldots$ & $\ldots \ldots \ldots$ & $2.6-21.6$ & 8.8 & & 22 \\
\hline $4.2-10.8$ & $6.2^{*}$ & $9.6-17.4$ & $13.5^{*}$ & $0.2-8.1$ & $5.3^{*}$ & & 23 \\
\hline ........................ & $\ldots \ldots \ldots$ & $\ldots$ & $\ldots \ldots \ldots$ & 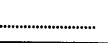 & 10.0 & …………………... & 24 \\
\hline
\end{tabular}

literature fall within the range noted for the present experiments. The lowest mean corpuscular volume observed was 58.2 cubic microns; the highest, 81.2 cubic microns; the mean for all of the dogs, 69.8 cubic microns. There was no apparent difference between the males and females with respect to mean corpuscular volume.

Considerable variation was observed in the leucocyte counts of the different animals. The females' counts ranged from 3.85 to 19.33 thousands per cubic millimeter; the males, from 3.93 to 21.34 thousands. The group mean, 8.91 thousands, agrees well with the mean normal figure, 8.00 thousands, suggested by Burnett. The means reported by Kohanawa (1928), 17.10 thousands; by Musser and Krumbhaar (1914), 15.92 thousands ; by Dawson (1900-01), 19.30 thousands; and by Goodall (1910), 19.50 thousands appear to be unusually high.

The inclusion of data on 18 additional male dogs did not alter significantly the mean values reported earlier for physical or chemical measurements on blood of normal dogs (Leichsenring, Biester, et al., 1932). 
Table 5. Differential White Cell Counts on the Blood of Normal Dogs (5 Males)

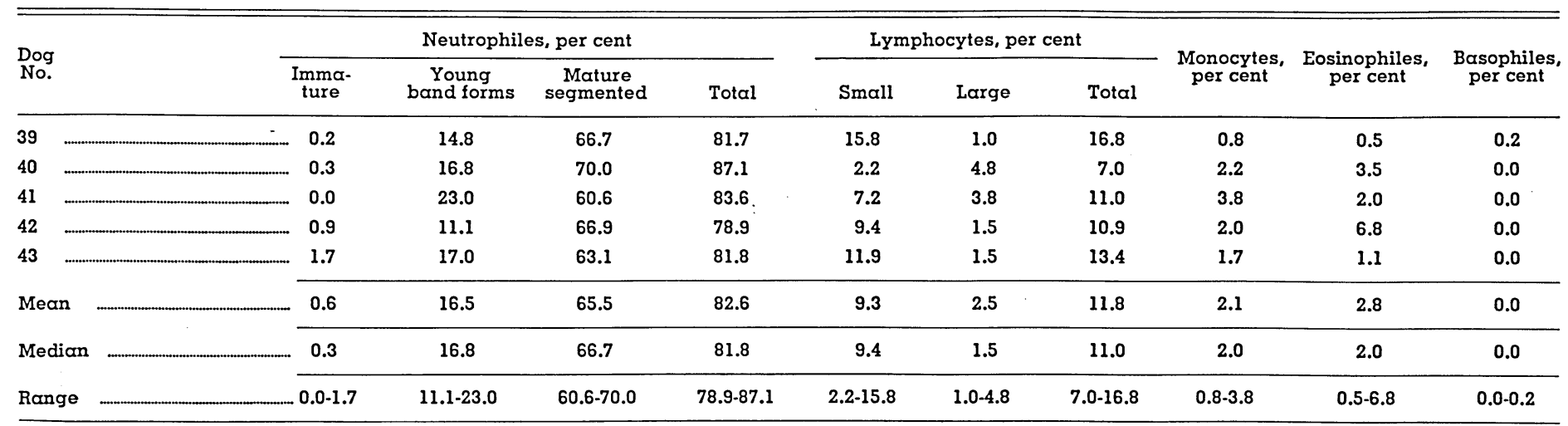


Table 5 shows the results of the differential white cell count for the five male animals on which this determination was made. The dogs involved showed a somewhat higher mean percentage of neutrophiles and a lower percentage of lymphocytes and monocytes than have been reported by other investigators (table 4 ):

\section{CHEMICAL MEASUREMENTS}

Tables 6 and 7 give data on various whole blood and plasma measurements. As with physical measurements, the values reported in most cases represent the mean of two or more duplicate or triplicate determinations. Evidently there was no essential difference, owing to sex, in the levels of the various constituents of whole blood. For both groups, widely ranging values were observed for the oxygen-combining capacity of the blood in volumes per cent. For the females, these values ranged from 13.2 to 22.9 volumes per cent; for the males, from 11.2 to 24.1 volumes per cent. The females' mean, 17.8 volumes per cent, was nearly identical with the males' mean, 17.4 volumes per cent. Total nitrogen in the blood was in a large degree influenced by the hemoglobin level. The means, medians, and ranges for the males and females were quite similar. The nonprotein nitrogen of whole blood also varied considerably, animal to animal, from 0.030 to $0.048 \mathrm{~g}$. per $100 \mathrm{cc}$. for the females, with a mean of 0.036 gram; and from 0.020 to $0.042 \mathrm{~g}$. for the males, with a mean of 0.034 gram. Although few data, covering urea nitrogen, amino acid nitrogen, and creatinine in whole blood were secured for the females, there appeared to be no sex difference in the levels of these particular blood constituents.

Table 6 shows a somewhat lower mean for oxygen-combining capacity for the animals used in the present investigation than values reported by most workers whose data are cited in the literature (table 8), although the mean of 17.5 volumes per cent is almost identical with that reported by Mayerson (1930) for his 60 canine subjects (17.4 volumes per cent). Except for the study by Van Slyke and Meyer (1913-14b), the literature offered no data covering the total nitrogen content of the blood. Data resulting from the present investigation seem to be in fair accord with values found in the literature for the nonprotein nitrogenous constituents considered.

In the consideration of the various plasma constituents, seven male dogs were observed. Table 7 presents the data acquired. Tables 9 and 10 were prepared to show the data reported in the literature covering the same plasma constituents and to permit comparison with the results of this research. It will be noted that there was no marked disagreement between the means obtained in the present investigation and those reported in the literature, and the ranges for the various values were also of the same magnitude. 
Table 6. Chemical Measurements on the Blood of Normal Dogs (13 Females, 33 Males)

\begin{tabular}{|c|c|c|c|c|c|c|c|}
\hline $\begin{array}{l}\text { Dog } \\
\text { No. }\end{array}$ & $\begin{array}{l}\text { Oxygen- } \\
\text { combining } \\
\text { capacity, } \\
\text { in } \\
\text { volumes } \\
\text { per } \\
\text { cent }\end{array}$ & $\begin{array}{c}\text { Total } \\
\text { nitrogen } \\
\text { in } \\
\text { whole } \\
\text { blood, } \\
\text { g. per } \\
100 \text { cc. }\end{array}$ & $\begin{array}{c}\text { Non- } \\
\text { protein } \\
\text { nitrogen } \\
\text { in whole } \\
\text { blood, } \\
\text { g. per } \\
\text { loo cc. }\end{array}$ & $\begin{array}{c}\text { Protein } \\
\text { nitrogen } \\
\text { in whole } \\
\text { blood, } \\
\text { g. per. } \\
100 \text { cc. }\end{array}$ & $\begin{array}{c}\text { Urea } \\
\text { nitrogen } \\
\text { in whole } \\
\text { blood, } \\
\text { mg. per } \\
100 \text { cc. }\end{array}$ & $\begin{array}{c}\text { Amino } \\
\text { acid } \\
\text { nitrogen } \\
\text { in whole } \\
\text { blood, } \\
\text { mg. per } \\
100 \text { cc. }\end{array}$ & $\begin{array}{l}\text { Creatin- } \\
\text { ine in } \\
\text { whole } \\
\text { blood, } \\
\text { mg. per } \\
100 \text { cc. }\end{array}$ \\
\hline \multicolumn{8}{|l|}{ Females } \\
\hline 1 & 21.2 & 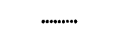 & .............. & $\ldots \ldots \ldots$ & $\ldots \ldots \ldots$ & 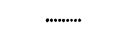 & $\ldots$ \\
\hline 2 & 21.6 & $\ldots \ldots . . . \cdot$ & $\ldots . . . . . . . .$. & $\ldots \ldots$ & 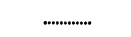 & .......... & $\ldots . . . . .$. \\
\hline 3 & 21.4 & $\ldots \ldots . .$. & .............. & $\ldots \ldots . .$. & $\ldots . . . . . . . . .$. & $\ldots . . . . . .$. & $\ldots . . . . . . .$. \\
\hline 15 & 18.3 & 2.89 & 0.039 & 2.85 & $\ldots$ & $\ldots . . . . .$. & $\ldots . . . . . . .$. \\
\hline 16 & 14.9 & 3.23 & 0.048 & 3.18 & $\ldots \ldots \ldots$ & $\ldots \ldots . . .$. & $\ldots \ldots . . .$. \\
\hline 19 & 22.9 & 3.50 & 0.034 & 3.46 & 15.38 & 4.05 & 1.72 \\
\hline 20 & 13.2 & 2.89 & 0.030 & 2.86 & 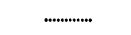 & $\ldots \ldots . .$. & $\ldots \ldots . .$. \\
\hline 21 & 14.1 & 3.18 & 0.030 & 3.15 & 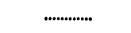 & $\ldots . . . . .$. & $\ldots \ldots \ldots$ \\
\hline 22 & 15.7 & 3.03 & 0.039 & 2.99 & 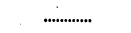 & $\ldots \ldots . .$. & $\ldots \ldots \ldots$ \\
\hline 25 & 15.6 & 3.22 & 0.032 & 3.19 & $\ldots . . . . . . .$. & $\ldots \ldots \ldots$ & $\ldots \ldots \ldots . .$. \\
\hline 26 & 15.2 & 3.06 & 0.041 & 3.02 & $\ldots \ldots \ldots$ & ........... & $\ldots . . . . .$. \\
\hline 29 & 18.9 & 3.16 & 0.030 & 3.13 & 16.50 & 11.58 & 1.54 \\
\hline 32 & 17.8 & 3.27 & 0.039 & 3.23 & 7.41 & .......... & $\ldots \ldots \ldots$ \\
\hline Mean & 17.8 & 3.14 & 0.036 & 3.11 & 13.10 & 7.82 & 1.63 \\
\hline Median & 17.8 & 3.18 & 0.039 & 3.15 & 15.38 & $\ldots \ldots \ldots$ & $\ldots \ldots \ldots$ \\
\hline Range & $13.2-22.9$ & $2.89-3.50$ & $0.030-0.048$ & $2.85-3.46$ & $7.41-16.50$ & $4.05-11.58$ & $1.54-1.72$ \\
\hline
\end{tabular}

\begin{tabular}{|c|c|c|c|c|c|c|c|}
\hline \\
\hline \multicolumn{8}{|l|}{7} \\
\hline 8 & 20.0 & $\ldots+. .$. & $\ldots$ & $\ldots \ldots . . .$. & .............. & $\ldots$ & ........... \\
\hline 9 & 13.1 & 3.00 & 0.036 & 2.96 & $\ldots$ & $\ldots \ldots . . .$. & ........... \\
\hline 10 & 21.4 & 3.48 & 0.035 & 3.45 & $\ldots$ & 3.37 & $\ldots . . . . .$. \\
\hline 12 & 20.2 & $\ldots . . . .$. & .............. & ........... & ............... & ........... & $\ldots . . . . .$. \\
\hline 14 & 21.8 & 3.82 & 0.031 & 3.79 & 4.96 & $\ldots . . . .$. & 1.34 \\
\hline 17 & 18.6 & 3.42 & 0.029 & 3.39 & 9.34 & 4.84 & 1.47 \\
\hline 18 & 17.4 & 3.05 & 0.029 & 3.03 & 10.34 & $\ldots . . . . .$. & 1.65 \\
\hline 27 & 19.4 & 3.25 & 0.036 & 3.21 & 14.71 & 10.44 & 1.43 \\
\hline 28 & 24.1 & 3.52 & 0.020 & 3.50 & 6.26 & 18.68 & 1.36 \\
\hline 30 & 17.4 & 3.14 & 0.042 & 3.09 & 7.87 & $\ldots$ & ........... \\
\hline 31 & 16.4 & 3.36 & 0.042 & 3.32 & 7.88 & .............. & ........... \\
\hline 33 & 13.2 & 2.91 & 0.035 & 2.87 & 14.25 & $\ldots . . . . . . . . .$. & ............ \\
\hline 34 & 17.0 & 3.16 & 0.033 & 3.13 & 12.29 & .............. & .......... \\
\hline 35 & 16.2 & 3.12 & 0.041 & 3.08 & 8.95 & $\ldots$ & ........... \\
\hline 36 & 18.5 & $\ldots$ & $\ldots$ & $\ldots . . . \cdot \cdot$ & $\ldots-\ldots . . . .$. & $\ldots$ & .......... \\
\hline 37 & 18.0 & $\ldots . . . . .$. & $\ldots$ & $\ldots$ & $\ldots$ & ................... & ........... \\
\hline 38 & 18.0 & $\ldots$ & $\ldots$ & $\ldots \ldots$ & $\ldots . . . . . . . .$. & $\ldots . . . \cdots \cdot \ldots$ & ........... \\
\hline 39 & 13.8 & $\ldots . . . . .$. & ............... & ............ & ............... & .................... & ........... \\
\hline 40 & 14.6 & $\ldots . . . . .$. & $\ldots . . . . . . . . .$. & $\ldots . \cdots \cdots$ & $\ldots . . . . . . . . .$. & A.................... & ........... \\
\hline 41 & 15.5 & $\ldots . . . . .$. & .............. & $\ldots . . . . . .$. & $\ldots . . . . . . . . .$. & $\ldots . . . . . . . . .$. & ........... \\
\hline 42 & 11.7 & $\ldots . . . \cdots$ & ............. & $\ldots . . . . .$. & $\ldots+. . . . . .$. & $\ldots$ & .......... \\
\hline 43 & 11.2 & ........... & .............. & $\ldots$ & $\ldots$ & $\ldots$ & ........... \\
\hline 44 & 16.1 & ............ & $\ldots$ & $\ldots$ & $\ldots$ & $\ldots$ & ........... \\
\hline 45 & 18.1 & $\ldots$ & $\ldots$ & $\ldots \ldots$ & $\ldots$ & $\ldots$ & ........... \\
\hline 46 & 19.0 & $\ldots$ & $\ldots . . . . . . . .$. & $\ldots$ & $\ldots . . . . . . . .$. & $\ldots . . . . . . . .$. & ............ \\
\hline 47 & 17.1 & $\ldots . . . . .$. & ............... & ............ & ................ & ................ & ............ \\
\hline 54 & 18.3 & $\ldots . . . . . .$. & ............... & $\ldots . . . \cdots$ & $\ldots$ & $\ldots . . . . . . . . .$. & ........... \\
\hline 55 & 18.5 & $\ldots . . . . .$. & ............. & $\ldots$ & $\ldots . . . . . . . \cdot$ & $\ldots . . . . . . . .$. & $\ldots . . . . . . .$. \\
\hline 56 & 16.7 & $\ldots$ & 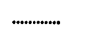 & $\ldots$ & $\ldots . . . . . .$. & $\ldots . . . \cdots \cdot \ldots$ & $\ldots$ \\
\hline 57 & 18.0 & $\ldots$ & $\ldots$ & $\ldots$ & 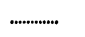 & $\ldots$ & $\ldots . . . . . .$. \\
\hline 58 & 14.9 & $\ldots . . . . .$. & .............. & $\ldots . . \cdots \cdot \cdot$ & $\ldots . . \cdots \cdots \cdots$ & ............... & $\ldots . . . \cdot \ldots$ \\
\hline 59 & 18.4 & $\ldots \ldots$ & $\ldots-\ldots . . . .$. & $\ldots \ldots . .$. & $\ldots$ & 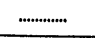 & $\ldots$ \\
\hline Mean & 17.4 & 3.27 & 0.034 & 3.24 & 9.68 & 9.33 & 1.45 \\
\hline Median & 18.0 & 3.25 & 0.035 & 3.21 & 9.34 & 10.44 & 1.43 \\
\hline Range & $.2-24.1$ & $.91-3.82$ & $0.020-0.042$ & $2.87-3.79$ & $4.96-14.71$ & $.37-18.68$ & $.34-1.65$ \\
\hline
\end{tabular}

Mean, all

dogs

17.5

3.21

0.035

3.18

10.47

8.83

1.50 
Table 7. Chemical Measurements on Plasma of Normal Dogs (7 males)

\begin{tabular}{|c|c|c|c|c|c|c|c|c|c|c|c|c|}
\hline $\begin{array}{l}\text { Dog } \\
\text { No. }\end{array}$ & $\begin{array}{c}\text { Total } \\
\text { nitrogen } \\
\text { in } \\
\text { plasma, } \\
\text { g. per } \\
100 \mathrm{cc} .\end{array}$ & $\begin{array}{c}\text { Non- } \\
\text { protein } \\
\text { nitrogen } \\
\text { in plasma, } \\
\text { g. per } \\
100 \mathrm{cc} .\end{array}$ & $\begin{array}{c}\text { Protein } \\
\text { nitrogen } \\
\text { in } \\
\text { plasma, } \\
\text { g.per } \\
\text { lo0 cc. }\end{array}$ & $\begin{array}{c}\text { Fibrin } \\
\text { in } \\
\text { plasma, } \\
\text { g. per } \\
100 \mathrm{cc} .\end{array}$ & $\begin{array}{c}\text { Albumin } \\
\text { in } \\
\text { plasma, } \\
\text { g. per } \\
100 \mathrm{cc} .\end{array}$ & $\begin{array}{c}\text { Globulin } \\
\text { in } \\
\text { plasma, } \\
\text { g.per } \\
100 \mathrm{cc} .\end{array}$ & $\begin{array}{l}\text { Total } \\
\text { phos- } \\
\text { phorus in } \\
\text { plasma, } \\
\text { mg. per } \\
\text { loo cc. }\end{array}$ & $\begin{array}{l}\text { Lipide } \\
\text { phos- } \\
\text { phorus in } \\
\text { plasma, } \\
\text { mg. per } \\
100 \text { cc. }\end{array}$ & $\begin{array}{l}\text { Inorganic } \\
\text { phos- } \\
\text { phorus in } \\
\text { plasma, } \\
\text { mg. per } \\
\text { loo cc. }\end{array}$ & $\begin{array}{l}\text { Total } \\
\text { cơlcium } \\
\text { in } \\
\text { plasma, } \\
\text { mg. per } \\
100 \text { cc. }\end{array}$ & $\begin{array}{l}\text { Diffus- } \\
\text { sible } \\
\text { calcium } \\
\text { in plasma, } \\
\text { mg. per } \\
100 \text { cc. }\end{array}$ & $\begin{array}{c}\text { Nondif- } \\
\text { fusible } \\
\text { calcium } \\
\text { in plasma } \\
\text { mg. per } \\
100 \text { cc. }\end{array}$ \\
\hline \multicolumn{13}{|l|}{ Males } \\
\hline 44 & 1.104 & 0.024 & 1.080 & 0.26 & . 3.91 & 2.26 & 25.9 & 17.1 & 6.01 & 13.46 & 8.52 & 4.98 \\
\hline 45 & 1.075 & 0.031 & 1.044 & 0.24 & 3.84 & 2.01 & 24.7 & 19.9 & 5.02 & 12.92 & 8.71 & 4.28 \\
\hline 46 & 1.269 & 0.032 & .1 .237 & 0.28 & 3.41 & 2.89 & 21.4 & 12.8 & 6.36 & 13.46 & 8.46 & 5.05 \\
\hline 47 & 1.103 & 0.037 & 1.066 & 0.47 & 4.60 & 2.36 & 21.9 & 16.7 & 4.14 & 10.18 & $6.75^{\circ}$ & 4.92 \\
\hline 54 & $\ldots$ & 0.033 & $\ldots$ & 0.30 & 3.88 & 2.02 & $\ldots . . . . .$. & $\ldots$ & $\ldots$ & $\ldots$ & $\ldots \ldots$ & $\ldots-\ldots . .$. \\
\hline 55 & $\ldots$ & 0.029 & $\ldots$ & 0.17 & 3.68 & 2.44 & $\ldots . . . . .$. & $\ldots \ldots$ & $\ldots . . . .$. & $\ldots-\ldots \ldots . . .$. & $\ldots-\ldots .$. & $\ldots . . . . .$. \\
\hline 56 & $\ldots$ & 0.031 & $\ldots+. . . . . .$. & 0.28 & 4.25 & 3.10 & $\ldots . . .$. & $\ldots . . .$. & $\ldots$ & $\ldots$ & $\ldots$ & $\ldots . . . \cdots$ \\
\hline Mecm & 1.138 & 0.031 & 1.107 & 0.29 & 3.94 & 2.44 & 23.5 & 16.6 & 5.38 & 12.50 & 8.11 & 4.81 \\
\hline Median & 1.104 & 0.031 & 1.080 & 0.28 & 3.88 & 2.36 & 24.7 & 17.1 & 6.01 & 13.46 & 8.52 & 4.98 \\
\hline Range & $1.075-1.269$ & $0.024-0.037$ & $1.044-1.237$ & $0.17-0.47$ & $3.41-4.60$ & $2.01-3.10$ & $21.4-25.9$ & 12.8-19.9 & $4.14-6.36$ & $10.18-13.46$ & $6.75-8.71$ & $4.28-5.05$ \\
\hline
\end{tabular}


Table 8. Chemical Measurements on the Blood of Normal Dogs Cited in the Literature

\begin{tabular}{|c|c|c|c|c|c|c|c|c|c|c|c|c|c|c|c|}
\hline & \multirow{2}{*}{$\begin{array}{l}\text { Reference } \\
\text { : }\end{array}$} & \multirow{2}{*}{$\begin{array}{l}\text { No. } \\
\text { of } \\
\text { dogs }\end{array}$} & \multicolumn{2}{|c|}{$\begin{array}{l}\text { Oxygen-combin- } \\
\text { ing capacity, } \\
\text { in volumes } \\
\text { per cent }\end{array}$} & \multicolumn{2}{|c|}{$\begin{array}{l}\text { Total } \\
\text { nitrogen in } \\
\text { whole blood, in } \\
\text { g. per } 100 \mathrm{cc} \text {. }\end{array}$} & \multicolumn{2}{|c|}{$\begin{array}{l}\text { Nonprotein } \\
\text { nitrogen in } \\
\text { whole blood, in } \\
\text { g. per } 100 \mathrm{cc} \text {. }\end{array}$} & \multicolumn{2}{|c|}{$\begin{array}{c}\text { Urea } \\
\text { nitrogen } \\
\text { in whole blood, } \\
\text { mg. per } 100 \mathrm{cc} .\end{array}$} & \multicolumn{2}{|c|}{$\begin{array}{l}\text { Amino acid } \\
\text { nitrogen in } \\
\text { whole blood, } \\
\text { mg. per } 100 \text { cc. }\end{array}$} & \multicolumn{3}{|c|}{$\begin{array}{l}\text { Creatinine } \\
\text { in whole } \\
\text { blood, mg. } \\
\text { per } 100 \mathrm{cc} .\end{array}$} \\
\hline & & & Range & Mean & Range & Mean & Range & Mean & Range & Mean & Range & Mean & Range & Mean & \\
\hline 1 & This study & $6-46$ & $11.2-24.1$ & 17.5 & $2.89-3.82$ & 3.21 & $0.020-0.048$ & 0.035 & $4.96-16.50$ & 10.47 & $3.37-18.68$ & 8.83 & $1.34-1.72$ & 1.50 & 1 \\
\hline 2 & Gibson et al. (1938) & 18 & $16.8-24.7$ & 20.27 & 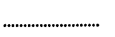 & $\ldots . . . \cdots . . . . .$. & 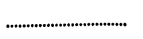 & $\ldots \ldots \ldots \ldots \ldots . . . .$. & 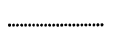 & $\ldots \ldots . . .$. & 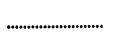 & $\ldots . . . . .$. & 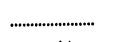 & $\ldots \ldots \ldots$ & 2 \\
\hline 3 & Bruner \& Wakerlin (1937) & 34 & $\ldots \ldots \ldots \ldots \ldots \ldots \ldots$ & $18.2 \dagger$ & 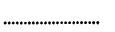 & $\ldots \ldots \ldots . . . . . .$. & ................................. & .................. & 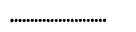 & $\ldots \ldots . . .$. & $\ldots$ & $\ldots . . . . .$. & $\ldots$ & $\ldots . . . . . .$. & 3 \\
\hline 4 & Wintrobe et al. (1935-36) & 54 & $\ldots$ & $19.6 \uparrow$ & 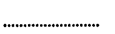 & $\ldots \ldots \ldots . . . .$. & 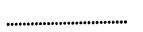 & $\ldots+\ldots \ldots \ldots . . . .$. & 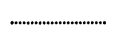 & $\ldots . . . . .$. & $\ldots$ & $\ldots \ldots \ldots . .$. & $\ldots$ & $\ldots \ldots \ldots$ & 4 \\
\hline 5 & Ashley \& Guest (1934) & 43 & 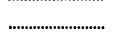 & $21.4 \uparrow$ & 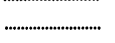 & $\ldots . . . \cdots \cdots$ & $\ldots$ & 0.035 & 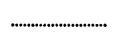 & $\ldots \ldots .$. & .......................... & $\ldots . \cdots \cdots \cdot$ & 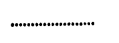 & $\ldots \ldots \ldots .$. & 5 \\
\hline 6 & Cahn \& Houget (1933) & 35 & & .............. & & $\ldots . . . \cdots \cdots \cdot . .$. & ... & ...................... & $3.7-12.6 \S$ & $\ldots \ldots \ldots .$. & $\ldots$ & $\ldots \ldots \ldots .$. & 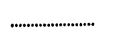 & $\ldots \ldots \cdots$ & 6 \\
\hline 7 & Camero \& Krumbhaar (1933) & 6 & $12.0-24.4$ & $20.0 \ddagger$ & & $\ldots . . . \cdots \cdots . .$. & .............................. & $\ldots \ldots \ldots \ldots \ldots$ & 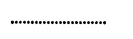 & $\ldots \ldots \ldots$ & 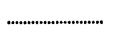 & $\ldots \ldots \ldots .$. & $\ldots+\cdots \cdots \cdots \cdots \cdots \cdots \cdots$ & ........... & 7 \\
\hline 8 & Mayerson (1930) & 60 & 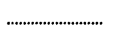 & $17.4 †$ & & ............... & 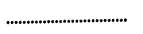 & $\ldots \ldots \ldots \ldots \ldots$ & $\ldots$ & $\ldots \ldots \ldots$ & $\ldots$ & $\ldots . . \cdots \cdots$ & 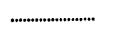 & $\ldots \ldots+\cdots$ & 8 \\
\hline 9 & Powers et al. (1930) & 25 & $13.8-27.2$ & $21.2 \ddagger$ & & $\ldots . . . . . . . .$. & ………………........ & .................. & $\ldots \ldots \ldots \ldots \ldots \ldots \ldots$ & $\ldots \ldots \ldots$ & 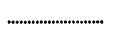 & $\ldots . . . . .$. & 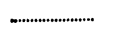 & $\ldots \ldots \ldots$ & 9 \\
\hline 10 & Stucky \& Rose (1929) & 10 & $15.2-23.6$ & $20.2 \ddagger$ & & $\ldots . . . \cdots \cdots \cdots$ & 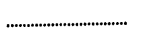 & $\ldots$ & $\ldots$ & $\ldots \ldots . .$. & $\ldots$ & $\ldots . . . .$. & 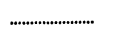 & $\ldots \cdots \cdots$ & 10 \\
\hline 11 & Teel \& Watkins (1929) & 2 & & .............. & & ............... & $0.028-0.042$ & $0.036^{*}$ & $\ldots \ldots \ldots \ldots \ldots$ & $\ldots \ldots \ldots$ & 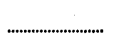 & $\ldots . . . . .$. & $1.2-1.5$ & $1.3^{*}$ & 11 \\
\hline 12 & Pierce \& Plant (1928) & 16 & $16.2-20.7 \mp$ & $\ldots \ldots \ldots . . . . .$. & & $\ldots . . . \cdots \cdots \cdots . . .$. & $\ldots \ldots \ldots \ldots \ldots \ldots \ldots \ldots$ & $\ldots \ldots \ldots \ldots \ldots . . .$. & $\ldots$ & $\ldots . . . . .$. & $\ldots$ & $\ldots . . . . .$. & 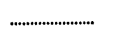 & $\ldots \ldots \ldots$ & 12 \\
\hline 13 & Emmons (1927-28) & $\ldots . .$. & . & $20.8 \$$ & & $\ldots . . . \cdots \cdots . . .$. & & $\ldots \ldots \ldots \ldots \ldots \ldots . .$. & & $\ldots \ldots . .$. & 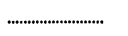 & $\ldots \ldots \ldots .$. & $\ldots \ldots \ldots \ldots \ldots \ldots \ldots$ & $\ldots . . \cdots \cdots$ & 13 \\
\hline 14 & Arnold \& Mendel (1927) & 3 & ……-................ & .............. & & $\ldots \ldots \ldots \ldots$ & $0.035-0.040$ & $0.037^{*}$ & & $\ldots . . . \cdot . \cdot$ & $\ldots$ & $\ldots . . . . .$. & 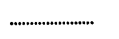 & $\ldots \ldots \ldots .$. & 14 \\
\hline 15 & Klieneberger (1927) & 6 & $21.2-33.9$ & 25.47 & & .............. & ............................. & $\ldots \ldots \ldots \ldots \ldots \ldots$ & 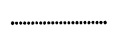 & $\ldots . . . .$. & & $\ldots . \cdots \cdots \cdot$. & $\ldots$ & $\ldots . . \cdots \cdot \cdot$ & 15 \\
\hline 16 & Collip et al. (1925) & 4 & & 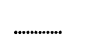 & & .............. & $0.026-0.043$ & $0.038^{*}$ & $10.0-16.0$ & $13.5^{\star}$ & & 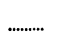 & & $\ldots . . . . .$. & 16 \\
\hline 17 & Haden \& Orr (1925) & 200 & 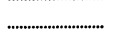 & ............... & & ............... & $0.016-0.078$ & 0.031 & $5.1-42.1$ & 11.7 & $2.0-14.6$ & $6.7 \mathbb{I}$ & $0.5-2.6$ & 1.5 & 17 \\
\hline 18 & Fisher \& Larson (1925) & 5 & (n.................. & $\ldots \ldots \ldots+\cdots$ & 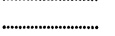 & $\ldots . . . \cdots \cdots . . .$. & $0.038-0.049$ & $0.040^{*}$ & 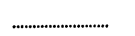 & $\ldots \ldots . . .$. & $\ldots \ldots \ldots \ldots \ldots \ldots \ldots \ldots$ & $\ldots . . . . .$. & $\ldots . . . \cdots \cdots \cdots \cdots$ & $\ldots . . \cdots \cdot \cdot$ & 18 \\
\hline 19 & Seth \& Luck (1925) & 1 & & 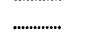 & & $\ldots . . \cdots \cdots \cdots . . .$. & ................................ & 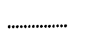 & .............................. & 7.7 & ................................ & 5.9 & .......................... & ........... & 19 \\
\hline 20 & Morgulis \& Edwards (1924) & 6 & 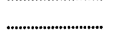 & $\cdots . . . . . . . . . .$. & & $\ldots . . . \cdots \cdots$ & $0.032-0.040$ & $0.035^{*}$ & $11.1-15.1$ & $12.9^{\star}$ & $7.8-9.1$ & $8.7^{\star}$ & $1.5-1.7$ & $1.6^{\star}$ & 20 \\
\hline 21 & Haden \& Orr (1923) & 25 & & $\ldots \ldots \ldots \ldots . .$. & & $\ldots . . . . . . . . .$. & $0.020-0.052$ & 0.030 & $5.1-26.1$ & 11.1 & $4.9-7.7$ & 6.3 & $1.1-2.1$ & 1.5 & 21 \\
\hline 22 & Haden \& Orr (1923) & 6 & $\ldots \ldots \ldots \ldots \ldots \ldots . . . . .$. & $\ldots \ldots \ldots \ldots . . .$. & ........................... & .............. & $0.022-0.039$ & $0.031^{\star}$ & $\begin{array}{ll}7.0 & -23.4\end{array}$ & $13.1^{\star}$ & $5.3-8.1$ & $6.6^{*}$ & $1.2-1.6$ & $1.3^{*}$ & 22 \\
\hline 23 & Scheunert \& v. Pelchrzim (1923) & 2 & 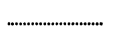 & .................. & ............................. & .................. & ................................ & $\ldots . . . \cdots \cdots \cdots \cdots \cdots$ & $11.1-16.7$ & $14.1^{*}$ & ................................. & $\ldots \ldots \ldots . .$. & $1.2 \cdot 1.5$ & $1.3^{*}$ & 23 \\
\hline 24 & Okada \& Hayashi (1922) & $\ldots . .$. & & 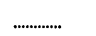 & ............................ & ............... & ……………........... & .................. & ........................... & $\ldots \ldots . . .$. & $6.3-8.8$ & 7.3 & 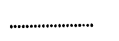 & $\ldots \ldots \ldots .$. & 24 \\
\hline 25 & Whipple \& Robscheit (1921) & 7 & $19.6-25.0$ & $22.7 \ddagger$ & ............................ & $\ldots . . . \cdots \cdots . . .$. & 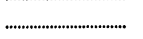 & 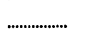 & 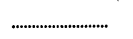 & $\ldots \ldots \ldots$ & 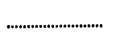 & $\ldots \ldots \ldots .$. & 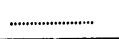 & $\ldots . . \cdots \cdots$ & 25 \\
\hline
\end{tabular}


Table 8-Continued

\begin{tabular}{|c|c|c|c|c|c|c|c|c|c|c|c|c|c|c|c|}
\hline & \multirow[t]{2}{*}{ Reference } & \multirow[t]{2}{*}{$\begin{array}{l}\text { No. } \\
\text { of } \\
\text { dogs }\end{array}$} & \multicolumn{2}{|c|}{$\begin{array}{l}\text { Oxygen-combin- } \\
\text { ing capacity, } \\
\text { in volumes } \\
\text { per cent }\end{array}$} & \multicolumn{2}{|c|}{$\begin{array}{c}\text { Total } \\
\text { nitrogen in } \\
\text { whole blood, in } \\
\text { g. per } 100 \mathrm{cc} \text {. }\end{array}$} & \multicolumn{2}{|c|}{$\begin{array}{l}\text { Nonprotein } \\
\text { nitrogen in } \\
\text { whole blood, in } \\
\text { g. per } 100 \mathrm{cc} \text {. }\end{array}$} & \multicolumn{2}{|c|}{$\begin{array}{c}\text { Urea } \\
\text { nitrogen } \\
\text { in whole blood, } \\
\text { mg. per } 100 \mathrm{cc} \text {. } \\
\end{array}$} & \multicolumn{2}{|c|}{$\begin{array}{c}\text { Amino acid } \\
\text { nitrogen in } \\
\text { whole blood, } \\
\text { mg. per } 100 \text { cc. }\end{array}$} & \multicolumn{3}{|c|}{$\begin{array}{l}\text { Creatinine } \\
\text { in whole } \\
\text { blood, mg. } \\
\text { per } 100 \mathrm{cc} \text {. }\end{array}$} \\
\hline & & & Range & Mean & Range & Mean & Range & Mean & Range & Mean & Range & Mean & Range & Mean & \\
\hline 26 & Austin et al. (1921) & 2 & 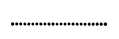 & $\ldots \ldots \ldots \ldots$ & $\ldots \ldots \ldots \ldots \ldots \ldots \ldots \ldots$ & $\ldots \ldots \ldots \ldots$ & $\ldots \ldots \ldots \cdots \cdots \cdots \cdots$ & 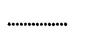 & $9.2-25.6$ & $13.8^{\star}$ & ............... & $\ldots . . . . .$. & $\ldots$ & $\ldots \ldots \ldots$ & 26 \\
\hline 27 & $\begin{array}{l}\text { Whipple, Hooper \& Robscheit } \\
\text { (1920) }\end{array}$ & 12 & $22.1-34.6$ & $27.9 \ddagger$ & & & & & & & & & & & \\
\hline 28 & Whipple, Hooper \& Robscheit & $\$ 4$ & $220.2-620.0$ & 210. & & & & & & $\cdots \cdots \cdots$ & & $\cdots \cdots \cdots$ & & $\cdots \cdots \cdots \cdots$ & 27 \\
\hline & $(1920)$ & 15 & $15.5-30.7$ & $23.9 \$$ & & $\ldots . . . \cdots \cdots \cdot \ldots$ & & & & 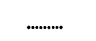 & & & & & 28 \\
\hline 29 & Fritsch (1920) & 10 & 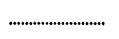 & $21.2 \dagger$ & 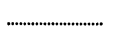 & $\ldots . . \cdots \cdots \cdot . .$. & 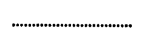 & $\ldots \ldots \ldots . . . . . . .$. & ............................. & $\ldots . . . . .$. & 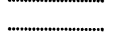 & $\ldots \ldots . . . .$. & & $\ldots \ldots . . .$. & 29 \\
\hline 30 & Kuhl (1919) & 10 & $\ldots+\ldots \ldots \ldots \ldots \ldots$ & $21.2 †$ & $\ldots \ldots \ldots+\cdots \cdots \cdots \cdots \cdots \cdots$ & $\ldots \cdots \cdots \cdots \cdots$ & 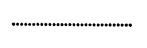 & $\ldots \ldots \ldots \ldots \ldots$ & 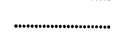 & $\ldots \ldots \ldots$ & 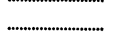 & $\ldots+\cdots$ & & $\cdots \cdots \cdots$ & 30 \\
\hline 31 & Bock (1917) & $\ldots . .$. & 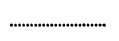 & $\ldots \ldots \ldots \ldots . .$. & & $\ldots . . . . . . .$. & & & $\ldots$ & $\ldots \ldots . .$. & $6.7-8.4$ & 7.5 & & $\ldots . . . . . .$. & 31 \\
\hline 32 & Draper (1917) & 1 & 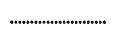 & $\ldots \ldots \ldots . . .$. & $\ldots \ldots \ldots \ldots \ldots \ldots \ldots$ & $\ldots \ldots \ldots \ldots . .$. & 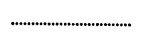 & 0.044 & 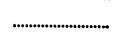 & 18.0 & 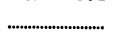 & $\ldots \ldots . . .$. & & 0.1 & 32 \\
\hline 33 & Wilson \& Plass (1917) & 1 & 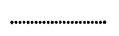 & $\ldots \ldots \ldots . . . .$. & $\ldots+\cdots \cdots \cdots \cdots \cdots$ & $\ldots . \cdots \cdots \cdots$ & 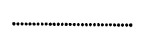 & $\ldots \ldots \ldots \ldots \ldots . . .$. & & $\ldots . . . . .$. & 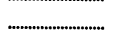 & $\ldots$ & & 1.7 & 33 \\
\hline 34 & Bang $(1915-16)$ & $\ldots . .$. & 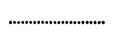 & $\ldots \ldots \ldots \ldots . .$. & $\ldots+\cdots \cdots+\cdots \cdots \cdots+\cdots$ & $\ldots . . \cdots \cdots \cdots . .$. & .............................. & 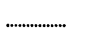 & $17.0-27.0$ & $\ldots . . . . .$. & $8.0-18.0$ & $\ldots \ldots \ldots$ & ........................ & $\ldots \ldots \ldots . . .$. & 34 \\
\hline 35 & György \& Zunz (1915) & 27 & $\ldots+\cdots \cdots \cdots \cdots \cdots$ & .............. & 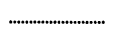 & $\ldots \ldots \ldots \ldots . .$. & ................................ & 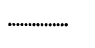 & $\ldots \ldots \ldots \ldots \ldots \cdots \cdots \cdots \cdots$ & $\ldots \ldots . .$. & $4.0-5.8$ & $4.7^{\star}$ & 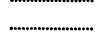 & 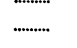 & 35 \\
\hline 36 & Taylor \& Lewis (1915) & 4 & .......................... & $\ldots+\cdots \cdots \cdots$ & $\ldots$ & $\ldots \ldots \ldots \ldots . .$. & $0.018-0.030$ & $0.026^{\star}$ & $6.9-22.6$ & $12.8^{\star}$ & & $\ldots . . . . . . .$. & & & 36 \\
\hline 37 & Marshall \& Davis (1914) & 1 & $\ldots$ & $\ldots \ldots \ldots$ & $\ldots+\cdots \cdots \cdots \cdots \cdots \cdots$ & $\ldots \ldots \ldots \ldots$ & ............................... & $\ldots . . . . . . . . . .$. & $12.6-13.1 \S$ & $12.8^{*}, \S$ & 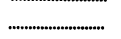 & $\ldots \ldots . . . .$. & .......... & $\ldots \ldots . . .$. & 37 \\
\hline 38 & Musser \& Krumbhaar (1914) & 47 & $17.2-23.3$ & $20.8 \mp$ & 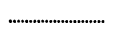 & $\ldots \ldots \ldots \ldots$ & ............................ & $\ldots \ldots \ldots \ldots \ldots \ldots$ & 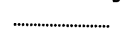 & $\ldots \ldots . . . .$. & ............................ & $\ldots . . . . . . .$. & & $\ldots . . . . .$. & 38 \\
\hline 39 & Shaffer (1914) & $\cdots \cdots$ & ............................. & ............... & .................................. & ............... & 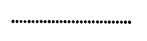 & 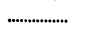 & $\ldots \cdots \cdots \cdots \cdots \cdots \cdots$ & $\ldots \ldots \ldots$ & $\ldots$ & $\ldots \ldots . .$. & $0.8-1.6$ & $1.2^{*}$ & 39 \\
\hline 40 & Van Slyke \& Meyer (1912) & 8 & 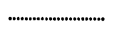 & ................ & ............. & 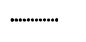 & & 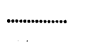 & $\ldots \ldots \ldots \ldots \ldots \ldots \ldots$ & $\ldots \ldots . .$. & $3.1-5.4$ & $4.4^{*}$ & $\ldots \ldots+\ldots \ldots \ldots \ldots$ & 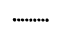 & 40 \\
\hline 41 & Van Slyke \& Meyer (1913-14a) & 3 & ...................... & $. . . \cdots \cdots \cdots . . .$. & & $\ldots$ & & $\ldots \ldots \ldots \ldots \ldots$ & & $\ldots \ldots \ldots \ldots$ & $3.9-5.9$ & $5.1^{\star}$ & & & 41 \\
\hline 42 & Van Slyke \& Meyer (1913-14b) & 5 & 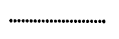 & $\ldots . \cdots \cdots \cdots \cdots$ & $3.32-3.99$ & $3.47^{\star}$ & & $\ldots$ & $5.0-14.0$ & $9.3^{*}$ & $3.9-10.0$ & $6.1^{*}$ & & $\ldots . . . . .$. & 42 \\
\hline 43 & Costantino (1913) & 1 & 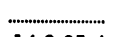 & $\ldots \ldots+\ldots . .$. & 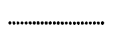 & $\ldots \ldots \ldots \ldots$ & & 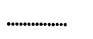 & 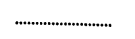 & $\ldots \ldots \ldots$ & $\ldots \ldots \ldots \ldots \ldots \ldots \ldots$ & 5.2 & & $\ldots . . .+. .$. & 43 \\
\hline 44 & Furno (1913) & 24 & $14.3-25.4$ & $19.2 \ddagger$ & $\ldots+\cdots \cdots \cdots \cdots \cdots \cdots \cdots$ & $\ldots . \cdots \cdots \cdots . .$. & ................................... & $\ldots$ & 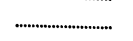 & $\ldots . . . .$. & 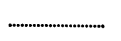 & $\ldots \ldots . .$. & & $\ldots . . . . .$. & 44 \\
\hline 45 & Plesch (1909) & 5 & 19.3-23.8 & $21.3^{*}$ & & $\ldots \ldots \ldots \ldots$ & & $\ldots . . . \cdots \cdots \cdots \cdots . . .$. & $\cdots \cdots$ & $\ldots$ & 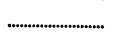 & 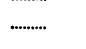 & & $+\cdots$ & 45 \\
\hline 46 & Breuer \& v Seiller (1903) & 3 & $23.6-26.4$ & $25.2 \dagger$ & & $\ldots \ldots \ldots . . .$. & & & & $\ldots \ldots \ldots . .$. & & & & & 46 \\
\hline 47 & Dawson $(1900-01)$ & 12 & $12.5-20.8$ & $15.9 \ddagger$ & & $\ldots . . . \cdots \cdot \ldots$ & ................................. & & $\ldots+\cdots \cdots \cdots$ & $\ldots . . . . .$. & ............................... & $\ldots$ & & $\ldots$ & 47 \\
\hline 48 & Otto (1885) & 17 & $16.2-21.4$ & $18.8+$ & ......................... & $\ldots-\ldots \ldots \ldots . .$. & 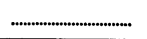 & $\ldots \ldots \ldots . . . . . . .$. & 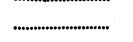 & $\ldots \ldots+\cdots$ & 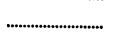 & $\ldots . . .$. & & $\ldots$ & 48 \\
\hline
\end{tabular}


Table 9. Chemical Measurements on Plasma of Normal Dogs Cited in the Literature (Nitrogenous Constituents)

\begin{tabular}{|c|c|c|c|c|c|c|c|c|c|c|c|c|c|}
\hline & \multirow[t]{2}{*}{ Reference } & \multirow{2}{*}{$\begin{array}{l}\text { No. } \\
\text { of } \\
\text { dogs }\end{array}$} & \multicolumn{2}{|c|}{$\begin{array}{l}\text { Nonprotein } \\
\text { nitrogen } \\
\text { in plasma, } \\
\text { g. per } 100 \text { cc. }\end{array}$} & \multicolumn{2}{|c|}{$\begin{array}{c}\text { Protein } \\
\text { nitrogen } \\
\text { in plasma, } \\
\text { g. per } 100 \text { cc. }\end{array}$} & \multicolumn{2}{|c|}{$\begin{array}{l}\text { Fibrin } \\
\text { in } \\
\text { plasma, } \\
\text { g. per } 100 \text { cc. }\end{array}$} & \multicolumn{2}{|c|}{$\begin{array}{l}\text { Albumin } \\
\text { in } \\
\text { plasma, } \\
\text { g. per } 100 \text { cc. }\end{array}$} & \multicolumn{2}{|c|}{$\begin{array}{l}\text { Globulin } \\
\text { in } \\
\text { plasma, } \\
\text { g. per } 100 \text { cc. }\end{array}$} & \\
\hline & & & Range & Mean & Range & Mean & Range & Mean & Range & Mean & Range & Mean & \\
\hline 1 & This study & $4-7$ & $0.024-0.037$ & 0.031 & $1.044-1.237$ & 1.107 & $0.17-0.47$ & 0.29 & $3.41-4.60$ & 3.94 & $2.01-3.10$ & 2.44 & 1 \\
\hline 2 & McNaught et al. (1936) & 12 & 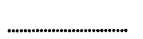 & $\ldots . . . . . . . . . .$. & $0.790-1.240 \mp$ & 0.930 味 & ............................ & $\ldots . . . \cdots \cdots \cdots$ & $3.18-4.28$ & 3.74 & $1.46-3.50$ & 2.05 & 2 \\
\hline 3 & Weech et al. (1935) & 19 & 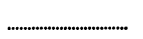 & $\ldots \ldots+\ldots . . .$. & ............................... & $1.018 \mp$ & 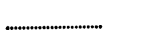 & ................ & $\ldots \ldots \ldots \ldots \ldots \ldots \ldots \ldots$ & 3.38 & 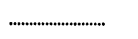 & 2.98 & 3 \\
\hline 4 & Turner \& Gibson (1932) & 4 & $\ldots$ & $\ldots \ldots \ldots \ldots . . .$. & $0.930-1.050 \mp$ & $\ldots . . . . . . . . .$. & .......................... & $\ldots \ldots \ldots \ldots . . . .$. & $\ldots$ & $\ldots \ldots \ldots \ldots . .$. & ............................... & $\ldots \ldots \ldots \ldots . . .$. & 4 \\
\hline 5 & Moore \& Stewart $(1930-31)$ & 1 & .............................. & $\ldots . . . . . . . . .$. & 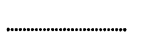 & $0.850 \mp$ & ........................... & $\ldots \ldots \ldots \ldots$ & $\ldots$ & 2.94 & $\ldots$ & 2.40 & 5 \\
\hline 6 & Vars $(1930)$ & 1 & 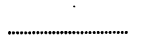 & $\ldots . . . . . . . .$. & ………...................... & $\ldots . . . . . . . . .$. & $0.38-0.42$ & $0.40^{*}$ & $\ldots+\cdots \cdots \cdots \cdots \cdots \cdots \cdots \cdots \cdots \cdots$ & $\ldots \ldots \ldots \ldots . .$. & $\ldots$ & 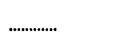 & 6 \\
\hline 7 & Matthew (1927) & 50 & $\ldots$ & $\ldots \ldots \ldots \ldots . .$. & $1.080-1.310$ & $1.180+$ 里 & $0.34-0.70 \mp$ & $0.46 \mp$ & 4.23-5.91‡ & $4.75 \ddagger$ & $1.82-2.97 \ddagger$ & $2.17 \ddagger$ & 7 \\
\hline 8 & Atkinson \& Ets (1922) & 6 & $0.021-0.038$ & 0.028 & ................................ & $\ldots . . . \cdots \cdots \cdots$ & $\ldots \ldots \ldots \ldots$ & $\ldots \ldots \ldots \ldots$ & 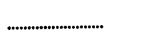 & $\ldots \ldots . . . . .$. & 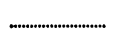 & $\ldots \ldots \ldots . . .$. & 8 \\
\hline 9 & Foster \& Whipple (1921-22) & 13 & …………................... & $\ldots \ldots \ldots \ldots . . .$. & $\ldots$ & $\ldots . . . \cdots \cdots \cdot . .$. & $0.31-0.51$ & 0.39 & $\ldots$ & $\ldots \ldots \ldots \ldots . .$. & 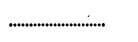 & $\ldots \ldots \ldots \ldots . .$. & 9 \\
\hline 10 & Smith et al. (1920) & 10 & $\ldots$ & $\ldots . . . . . . . . .$. & ................................ & $\ldots . . . \cdots \cdots \cdot \ldots$ & 0.17-0.77¥.\| & $0.34 \mp \cdot \|$ & 3.28-4.61屯.I & $3.78^{*}, \mathfrak{I}$ & $1.33-2.97 \pm \mathbb{I}$ & $1.84^{*} \cdot \pm \mathbb{I}$ & 10 \\
\hline 11 & Whipple (1914) & 11 & $\ldots$ & $\ldots \ldots \ldots . . . . .$. & $\ldots+\ldots \ldots \ldots \ldots \ldots \ldots$ & $\ldots$ & $0.20-0.87$ & 0.47 & $\ldots$ & $\ldots \ldots \ldots$ & ......................... & $\ldots . . . \cdots \cdots$ & 11 \\
\hline 12 & Morawitz (1906) & 7 & …………................... & $\ldots . . . . . . . . .$. & $0.780-0.980 \S$ & $0.890 \uparrow \cdot \S$ & $\ldots$ & $\ldots . . \cdots \cdots \cdots$ & $3.31-3.83$ & $3.55^{\star} \cdot \dagger$ & $1.28-2.71$ & $1.99^{\star}, \ddagger$ & 12 \\
\hline 13 & Lewinski (1903) & $\ldots . .$. & $\ldots$ & $\ldots \ldots \ldots . .$. & $\ldots \ldots \ldots \ldots \ldots \ldots \ldots \ldots$ & $0.960 †$ & 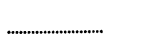 & 0.60 & 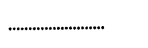 & 3.17 & 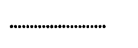 & 2.26 & 13 \\
\hline
\end{tabular}

* Calculated average.

† Protein nitrogen calculated from gms. of protein per 100 cc. plasma.

\# Calculated from percentage.

$\S$ Calculated from percentage protein in plasma.

II Determinations made on serum.

|| Twenty dogs. 
Table 10. Chemical Measurements on Plasma of Normal Dogs Cited in the Literature (Phosphorus and Calcium Fractions)

\begin{tabular}{|c|c|c|c|c|c|c|c|c|c|c|c|c|c|c|c|}
\hline & \multirow[t]{2}{*}{ Reference } & \multirow[t]{2}{*}{$\begin{array}{l}\text { No. } \\
\text { of } \\
\text { dogs }\end{array}$} & \multicolumn{2}{|c|}{$\begin{array}{c}\text { Total } \\
\text { phosphorus } \\
\text { in plasma, } \\
\text { mg. per } 100 \text { cc. }\end{array}$} & \multicolumn{2}{|c|}{$\begin{array}{c}\text { Lipide } \\
\text { phosphorus } \\
\text { in plasma, } \\
\text { mg. per } 100 \text { cc. }\end{array}$} & \multicolumn{2}{|c|}{$\begin{array}{c}\text { Inorganic } \\
\text { phosphorus } \\
\text { in plasma, } \\
\text { mg. per } 100 \text { cc. }\end{array}$} & \multicolumn{2}{|c|}{$\begin{array}{c}\text { Total } \\
\text { calcium } \\
\text { in plasma, } \\
\text { mg. per } 100 \text { cc. }\end{array}$} & \multicolumn{2}{|c|}{$\begin{array}{c}\text { Diffusible } \\
\text { calcium } \\
\text { in plasma, } \\
\text { mg. per } 100 \text { cc. }\end{array}$} & \multicolumn{3}{|c|}{$\begin{array}{l}\text { Nondiffusible } \\
\text { calcium } \\
\text { in plasma, } \\
\text { mg. per } 100 \text { cc. }\end{array}$} \\
\hline & & & Range & Mean & Range & Mean & Range & Mean & Range & Mean & Range & Mean & Range & Mean & \\
\hline 1 & This study & 4 & $21.4-25.9$ & 23.5 & 12.8-19.9 & 16.6 & $4.14-6.36$ & 5.38 & $10.18-13.46$ & 12.50 & $6.75-8.71$ & 8.11 & $4.28-5.05$ & 4.81 & 1 \\
\hline 2 & Ashley and Guest (1934) & $32-39$ & $\ldots$ & 16.2 & ............................ & $\ldots \ldots \ldots \ldots .$. & $\ldots+\cdots \cdots \cdots \cdots \cdots \cdots$ & 3.77 & $\ldots$ & 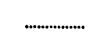 & 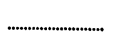 & & & & 2 \\
\hline 3 & Holt (1931-32) & $\ldots . . \cdot$ & ........................... & $\ldots+\cdots \cdots \cdots \cdot$ & 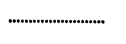 & $\ldots . . \cdots \cdots \cdots$ & .......................... & $\ldots \ldots \ldots \ldots$ & 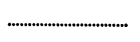 & 11.68 & 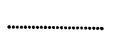 & $\ldots . . . . . . .$. & & & 3 \\
\hline 4 & Gerschman (1931) & 13 & $\ldots$ & ............... & 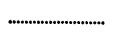 & $\ldots . \cdots \cdots$ & 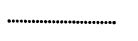 & 3.30 & 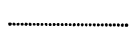 & $\ldots \ldots \ldots \ldots \ldots . . .$. & 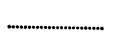 & $\ldots$ & 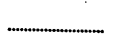 & ........ & 4 \\
\hline 5 & Brull (1930) & 5 & $\ldots$ & $\ldots$ & $\ldots$ & $\ldots$ & 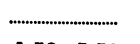 & $\ldots \ldots$ & $10.00-14.60$ & $12.10 \dagger$ & $6.60-9.20$ & 7.707 & $3.00-5.40$ & $4.40 \mp, \ddagger$ & 5 \\
\hline 6 & Briggs et al. (1923-24) & 7 & $\ldots$ & $\cdots \cdots \cdots \cdots \cdots$ & .......................... & $\ldots . . \cdots \cdots \cdot . .$. & $1.50-5.50$ & 3.00 & $9.80-11.60$ & 10.60 & 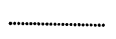 & $\ldots \ldots \ldots . . . .$. & & ........... & 6 \\
\hline 7 & Buell (1923) & 10 & $\ldots . . \cdots \cdots \cdots \cdots \cdots \cdots \cdots \cdots$ & $\ldots \ldots \ldots \ldots . .$. & 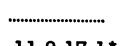 & $\ldots$ & $3.67-10.60^{*}$ & $4.55^{*}$ & ...................... & $\ldots . . . \cdots \cdots \cdots \cdots . . .$. & $\ldots$ & $\ldots . . . . . .$. & & & 7 \\
\hline 8 & Randles and Knudson (1922) & 2 & 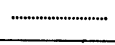 & $\ldots . . . \cdots \cdots \cdot \cdots$ & $11.2-17.1^{\star}$ & $14.3^{*}$ & .............................. & $\ldots . \cdots \cdots \cdots$ & ............................ & $\ldots \ldots \ldots \ldots \ldots$ & ............................ & $\ldots \ldots \ldots \ldots$ & & & 8 \\
\hline
\end{tabular}

* Calculated from mg. of $\mathrm{H}_{3} \mathrm{PO}_{4}$ per 100 cc. plasma.

$\uparrow$ Calculated average.

\# Calculated by difference from total calcium and diffusible calcium. 


\section{SUMMARY}

Normal values for the blood constituents of 50 adult dogs were obtained, and data acquired are reported in this bulletin.

The mean for the total blood volume was $76.4 \mathrm{cc}$. per kilogram of body weight; for cell volume, 36.2 cc. per kilogram of body weight; for cell volume per cent; 47.2 per cent; for red cell count, 6.83 millions per cubic millimeter; for red cell diameter, 6.98 microns; for mean corpuscular volume, 69.8 cubic microns; and for white cell count, 8.91 thousands per cubic millimeter.

The mean for oxygen-combining capacity was 17.5 volumes per cent; for total nitrogen, 3.21 grams; for nonprotein nitrogen, 0.035 gram; for protein nitrogen, 3.18 grams; for urea nitrogen, 10.47 milligrams; for amino acid nitrogen, 8.83 milligrams; and for creatinine, 1.50 milligrams per $100 \mathrm{cc}$. of whole blood.

The mean values obtained in the differential white cell count were 82.6 per cent for total neutrophiles; 11.8 per cent for total lymphocytes; 2.1 per cent for monocytes; 2.8 per cent for eosinophiles; and 0.0 per cent for basophiles.

The mean values for total nitrogen were 1.138 grams; for nonprotein nitrogen, 0.031 gram; for protein nitrogen, 1.107 grams; for fibrinogen, 0.29 gram; for albumin, 3.94 grams; for globulin, 2.44 grams ; for total phosphorus, 23.5 milligrams ; for lipide phosphorus, 16.6 milligrams; for inorganic phosphorus, 5.38 milligrams; for total calcium, 12.50 milligrams; for diffusible calcium, 8.11 milligrams; for nondiffusible calcium, 4.81 milligrams per $100 \mathrm{cc}$. of plasma.

No significant sex difference was observed in any of the physical or chemical measurements reported. 


\section{ERYTHROCYTES AND LEUCOCYTES}

\section{ERYTHROCYTE COUNTS}

A

S PREVIOUSLY OUTLINED, the present investigation consisted of both

a short-term and a long-term study for each of which different groups of dogs were used as experimental subjects.

The animals were maintained on the synthetic diet and fed in amounts calculated to keep them at reasonably constant body weight. Minimum, maximum, and mean weights for each of the animals are given in table 11. Mean weights were used in calculating total blood volume, plasma volume, and cell volume, in cubic centimeters per kilogram.

Table 11. Weights of Experimental Dogs, in Kilograms

\begin{tabular}{|c|c|c|c|c|}
\hline Dog No. & & Minimum & Maximum & Mean \\
\hline \multicolumn{5}{|c|}{ Short-term study } \\
\hline \multicolumn{5}{|l|}{ Males } \\
\hline 44 & ............................ & 22.3 & 23.5 & 22.9 \\
\hline 45 & 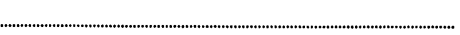 & 17.7 & 19.5 & 18.6 \\
\hline 46 & ........................ & 16.2 & 19.5 & 17.7 \\
\hline 47 & (1) & 16.8 & 21.3 & 18.9 \\
\hline Mean & 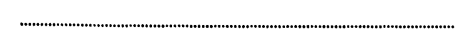 & 18.2 & 21.0 & 19.5 \\
\hline \multicolumn{5}{|c|}{ Long-term study } \\
\hline Females & & & & \\
\hline $20 \quad \ldots$ & ..................... & 17.7 & 19.7 & 18.9 \\
\hline 21 & 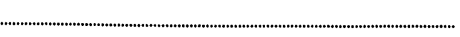 & 11.0 & 12.0 & 11.5 \\
\hline 22 & 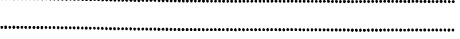 & 11.8 & 13.6 & 12.7 \\
\hline 19 & (1) & 17.7 & 18.6 & 18.1 \\
\hline 32 & (1) & 16.6 & 17.9 & 17.2 \\
\hline Mean & (1) & 15.0 & 16.4 & 15.7 \\
\hline \multicolumn{5}{|l|}{ Males } \\
\hline 17 & (1) & 16.3 & 17.0 & 16.8 \\
\hline 18 & (-1) & 20.4 & 21.1 & 20.7 \\
\hline 30 & a & 16.3 & 17.5 & 16.8 \\
\hline 31 & "1 & 20.3 & 21.3 & 20.7 \\
\hline Mean & 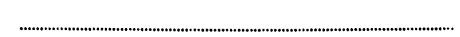 & 18.3 & 19.2 & 18.8 \\
\hline
\end{tabular}

For the 10 experiments comprising the short-term study, the mean red cell count before bleeding was 6.86 millions per cubic millimeter; the mean percentage of the total blood volume removed through bleeding and post-bleeding sampling was 26 per cent; and on the day after hemorrhage the mean red cell count was 5.46 millions per cubic millimeter, a decrease of 1.40 millions or 20 per cent (table 12). Red cell counts dropped steadily, during the period of daily sampling, to a mean 
Table 12. Values for Red Cell Counts, in Millions per Cubic Millimeter

(Short-term study)

\begin{tabular}{|c|c|c|c|c|c|c|}
\hline \multirow{2}{*}{$\begin{array}{l}\text { Dog } \\
\text { No. }\end{array}$} & \multirow{2}{*}{$\begin{array}{l}\text { Last pre- } \\
\text { bleeding }\end{array}$} & \multirow{2}{*}{$\begin{array}{l}\text { Within } \\
30 \text { minutes } \\
\text { of start of } \\
\text { bleeding }\end{array}$} & \multicolumn{4}{|c|}{ Post-bleeding period } \\
\hline & & & 1 day & 2 days & 3 days & 5-7 days \\
\hline Males & & & & & . & \\
\hline \multirow{2}{*}{44} & 7.04 & 5.48 & 5.24 & 5.12 & 4.64 & 4.83 \\
\hline & 6.26 & 5.54 & 5.10 & 4.68 & 4.56 & 4.96 \\
\hline \multirow[t]{2}{*}{45} & 7.84 & 7.06 & 6.48 & 5.94 & 5.93 & 5.42 \\
\hline & 8.04 & 7.27 & 6.56 & 6.12 & 5.61 & 5.56 \\
\hline \multirow[t]{2}{*}{46} & 5.52 & 5.53 & 5.00 & 4.84 & 4.61 & 4.72 \\
\hline & 6.34 & 6.24 & 4.84 & 5.38 & 4.69 & 3.90 \\
\hline \multirow[t]{4}{*}{47} & 6.80 & 6.23 & 5.15 & 5.12 & 5.32 & 5.32 \\
\hline & 6.54 & 5.24 & 4.96 & 5.32 & 5.18 & 4.90 \\
\hline & 7.17 & 6.01 & 5.81 & 5.47 & 5.14 & 4.86 \\
\hline & 7.06 & 6.08 & 5.44 & 5.69 & 4.88 & 4.30 \\
\hline Mean & 6.86 & 6.07 & 5.46 & 5.37 & 5.06 & 4.87 \\
\hline
\end{tabular}

level of 4.87 millions five to seven days after hemorrhage. These decreases are attributable to failure to replace the cells removed in daily sampling and to dilution resulting from increase in plasma.

In the long-term study, it was noted that in the females the mean red cell count had returned to the normal level, 6.89 millions per cubic millimeter, by the seventh week, and was somewhat above normal, 7.03 millions, by the twelfth week (table 13). In the males, the normal mean, 6.87 millions, had been regained in five weeks, and by the twelfth

Table 13. Values for Red Cell Counts, in Millions per Cubic Millimeter (Long-term study)

\begin{tabular}{|c|c|c|c|c|c|c|c|c|c|c|}
\hline \multirow{2}{*}{$\begin{array}{l}\text { Dog } \\
\text { No. }\end{array}$} & \multirow{2}{*}{$\begin{array}{l}\text { Pre- } \\
\text { bleed- } \\
\text { ing } \\
\text { normal }\end{array}$} & \multicolumn{9}{|c|}{ Post-bleeding period } \\
\hline & & 1-2 days & 1 wk. & 2 wks. & 3 wks. & 4 wks. & 5 wks. & 6 wks. & 7 wks. & 12 wks. \\
\hline \multicolumn{11}{|c|}{ Females } \\
\hline 20 & 6.98 & 5.64 & 7.51 & 4.98 & 5.85 & 7.63 & 5.62 & 5.66 & 6.28 & 7.02 \\
\hline 21 & 7.08 & 4.80 & 7.10 & 6.64 & 7.36 & 8.15 & 7.44 & 8.67 & 7.90 & $\ldots \ldots \ldots . . . .$. \\
\hline 22 & 6.18 & 4.54 & 4.89 & 6.75 & 6.01 & 6.76 & 7.54 & 7.02 & 7.89 & $\ldots \ldots \ldots \ldots . . .$. \\
\hline 19 & 7.00 & 5.06 & 4.98 & 5.74 & 5.66 & 6.11 & 6.38 & 5.88 & 6.68 & 8.08 \\
\hline 32 & 7.21 & 4.66 & 4.76 & 5.09 & 4.82 & 5.64 & 5.20 & 5.99 & 5.80 & 5.98 \\
\hline Mean & 6.89 & 4.94 & 5.85 & 5.84 & 5.94 & 6.86 & 6.43 & 6.64 & 6.91 & 7.03 \\
\hline \multicolumn{11}{|l|}{ Males } \\
\hline 17 & 7.89 & 5.73 & 6.15 & 7.10 & 6.97 & 7.88 & 7.44 & 8.04 & 7.44 & 7.46 \\
\hline 18 & 6.99 & 5.24 & 4.54 & 6.34 & 6.20 & 6.48 & 6.48 & 6.58 & 6.45 & 7.68 \\
\hline 30 & 6.14 & 5.35 & 5.31 & 5.99 & 5.93 & 6.50 & 7.08 & 6.62 & $\ldots . . . . . . . .$. & 7.40 \\
\hline 31 & 6.46 & 4.45 & 4.10 & 5.64 & 6.34 & 6.14 & 6.73 & 6.80 & $\ldots$ & 6.39 \\
\hline Mean & 6.87 & 5.19 & 5.02 & 6.27 & 6.36 & 6.75 & 6.93 & 7.01 & 6.94 & 7.23 \\
\hline
\end{tabular}


week the red cell count was five per cent above normal, 7.23 millions, despite weekly sampling. This superior response on the part of the males was apparent throughout the entire investigation.

\section{CELL SIZE}

Cell diameters were not measured during the short-term study. The calculation of mean corpuscular volume resulted in a mean value of 58.4 cubic microns ${ }^{1}$ for the last determination before hemorrhage, but it must be realized that this value does not represent the normal level based on initial measurements made on these experimental animals, since gradual decreases in mean corpuscular volume occurred throughout the study (table 14). For example, the normal value for dog 47 was 76.2 cubic microns; the last value before the first unit hemorrhage was 68.9 cubic microns. Throughout the four experiments on this animal there was a gradual decrease in mean corpuscular volume so that the last pre-bleeding value before the fourth unit hemorrhage on this animal was 45.6 cubic microns. The actual mean normal value for the four animals was 71.6 cubic microns.

Table 14. Values for Mean Corpuscular Volume, in Cubic Microns (Short-term study)

\begin{tabular}{|c|c|c|c|c|c|c|}
\hline \multirow{2}{*}{$\begin{array}{l}\text { Dog } \\
\text { No. }\end{array}$} & \multirow{2}{*}{$\begin{array}{l}\text { Last pre- } \\
\text { bleeding }\end{array}$} & \multirow{2}{*}{$\begin{array}{l}\text { Within } \\
30 \text { minutes } \\
\text { of start of } \\
\text { bleeding }\end{array}$} & \multicolumn{4}{|c|}{ Post-bleeding period } \\
\hline & & & I day & 2 days & 3 days & 5-7 days \\
\hline \multicolumn{7}{|l|}{ Males } \\
\hline \multirow[t]{2}{*}{44} & 54.4 & 60.2 & 59.6 & 55.5 & 56.0 & 57.4 \\
\hline & 56.2 & 62.2 & 61.1 & 65.6 & 58.9 & 60.9 \\
\hline 45 & 57.8 & 60.2 & 62.8 & 57.3 & 63.3 & 60.0 \\
\hline \multirow[t]{2}{*}{46} & 76.3 & 77.8 & 64.2 & 64.0 & 63.1 & 61.9 \\
\hline & 63.0 & 60.5 & 63.4 & 55.6 & 55.6 & 54.1 \\
\hline \multirow[t]{4}{*}{47} & 68.9 & 63.1 & 70.1 & 66.0 & 61.6 & 66.4 \\
\hline & 56.1 & 64.1 & 57.0 & 52.2 & 50.8 & 55.0 \\
\hline & 47.0 & 49.2 & 43.7 & 43.9 & 50.7 & 45.5 \\
\hline & 45.6 & 48.8 & 49.9 & 43.1 & 49.4 & 43.4 \\
\hline Mean & 58.4 & 60.7 & 59.1 & 55.9 & 56.6 & 56.1 \\
\hline
\end{tabular}

Determinations made 30 minutes after the start of hemorrhage showed a slight increase to 60.7 cubic microns as contrasted with the pre-bleeding mean of 58.4 cubic microns. This was followed, on the day after hemorrhage, by a mean of 59.1 cubic microns, with a decrease on the second day to 55.9 cubic microns, at which level the value remained practically unchanged during the balance of the experimental period.

${ }^{1}$ Based on nine experiments; one experiment, on dog No. 45, could not be included because of failure to secure a blood sample within 30 minutes of the start of bleeding. 
Table 15. Values for Red Cell Diameters, in Microns (Long-term study)

\begin{tabular}{|c|c|c|c|c|c|c|c|c|c|}
\hline \multirow{2}{*}{$\begin{array}{l}\text { Dog } \\
\text { No. }\end{array}$} & \multirow{2}{*}{$\begin{array}{c}\text { Pre- } \\
\text { bleeding } \\
\text { normal }\end{array}$} & \multicolumn{8}{|c|}{ Post-bleeding period } \\
\hline & & 1-2 days & $1 \mathrm{wk}$. & 2 wks. & 3 wks. & 4 wks. & 5 wks. & 6 wks. & 12 wks. \\
\hline Females & & & & & & & & & \\
\hline 19 & 6.90 & 6.88 & 6.84 & 6.51 & 6.43 & 6.12 & 6.21 & 6.24 & 5.87 \\
\hline 32 & 6.93 & 6.77 & 6.70 & 6.26 & 6.37 & 6.49 & 6.44 & 6.28 & 6.50 \\
\hline Mean & 6.92 & 6.82 & 6.77 . & 6.38 & 6.40 & 6.30 & 6.32 & 6.26 & 6.18 \\
\hline Males & & & & & & & & & \\
\hline 17 & 6.90 & 6.77 & 6.68 & 6.26 & 6.40 & 6.07 & 6.22 & 6.18 & 5.86 \\
\hline 18 & 6.80 & 6.57 & 6.50 & 6.28 & 6.22 & 6.37 & 6.21 & 6.20 & 5.87 \\
\hline 30 & 7.25 & 6.96 & 6.90 & 6.70 & 6.65 & 6.67 & 6.67 & 6.81 & 6.59 \\
\hline 31 & 7.03 & 7.03 & 6.72 & 6.77 & 6.70 & 6.60 & 6.56 & 6.55 & 6.51 \\
\hline Mean & 6.99 & 6.83 & 6.70 & 6.50 & 6.49 & 6.43 & 6.42 & 6.44 & 6.21 \\
\hline
\end{tabular}

During the long-term study, cell-diameter measurements were made on two of the females and on the four males. The mean pre-bleeding normal cell diameter of the females was found to be 6.92 microns (table 15). Following hemorrhage, cell diameter decreased gradually until at the end of the sixth week the mean for the females was 6.26 microns, a decrease of 10 per cent. No further decrease was noted although the animals were observed for an additional six-week interval.

For the males, the mean pre-bleeding normal cell diameter was found to be 6.99 microns. As with the females, cell diameter decreased following hemorrhage, until it reached a mean of 6.44 microns at the end of six weeks, a decrease of 9 per cent, and 6.21 microns at the end of 12 weeks, a decrease of 11 per cent.

It is evident that whereas the number of red blood cells increased

Table 16. Values for Mean Corpuscular Volume, in Cubic Microns (Long-term study)

\begin{tabular}{|c|c|c|c|c|c|c|c|c|c|}
\hline \multirow{2}{*}{$\begin{array}{l}\text { Dog } \\
\text { No. }\end{array}$} & \multirow{2}{*}{$\begin{array}{c}\text { Pre- } \\
\text { bleeding } \\
\text { normal }\end{array}$} & \multicolumn{8}{|c|}{ Post-bleeding period } \\
\hline & & 1-2 days & 1 wk. & 2 wks. & 3 wks. & 4 wks. & 5 wks. & 6 wks. & 12 wks. \\
\hline \multicolumn{10}{|c|}{ Females } \\
\hline 20 & 59.9 & 57.1 & 46.7 & 53.6 & 52.5 & 43.6 & 53.6 & 52.1 & 46.4 \\
\hline 21 & 63.3 & 66.4 & 55.8 & 54.5 & 57.9 & 52.8 & 52.8 & 46.2 & ................ \\
\hline 22 & 73.5 & 76.9 & 71.0 & 56.6 & 62.1 & 59.2 & 58.5 & 67.4 & ............... \\
\hline 19 & 81.4 & 69.6 & 78.9 & 68.1 & $\ldots \ldots \ldots \ldots$ & 59.9 & 58.3 & 62.4 & 43.4 \\
\hline 32 & 71.1 & 72.5 & 65.3 & 67.5 & 69.7 & 64.2 & 67.1 & 60.3 & 62.7 \\
\hline Mean & 69.8 & 68.5 & 63.5 & 60.1 & 60.5 & 55.9 & 58.1 & 57.7 & 50.9 \\
\hline \multicolumn{10}{|l|}{ Males } \\
\hline 17 & 64.9 & 71.2 & 57.6 & 50.0 & 60.4 & 49.1 & 52.4 & 46.5 & 46.9 \\
\hline 18 & 66.0 & 58.4 & 73.3 & 56.0 & 54.5 & 56.5 & 55.1 & 51.8 & 46.6 \\
\hline 30 & 65.6 & 58.3 & 62.5 & 62.8 & 67.4 & 65.5 & 64.1 & 65.0 & 62.6 \\
\hline 31 & 70.6 & 73.9 & $\ldots$ & 69.0 & 64.5 & 71.8 & 68.8 & 63.5 & 72.1 \\
\hline Mean & 66.8 & 65.4 & 64.5 & 59.4 & 61.7 & 60.7 & 60.1 & 56.7 & 57.0 \\
\hline
\end{tabular}


during the long-term study to a level considerably above normal, cell diameter decreased progressively, the females showing a more rapid decline than the males.

The pre-bleeding normal mean corpuscular volume for the five females used in this long-term study was 69.8 cubic microns (table 16). Following hemorrhage, there was a steady reduction in mean corpuscular volume until it reached a mean level of 57.7 cubic microns at the end of the sixth week, a decrease of 17 per cent. For the three females observed for 12 weeks, mean corpuscular volume at the end of that period was 50.9 cubic microns, contrasted with the mean level of 58.3 cubic microns found for the same animals at the end of the sixth postbleeding week.

For the males, the pre-bleeding normal mean corpuscular volume was 66.8 cubic microns. By the end of the sixth week following hemorrhage, this level had been reduced to 56.7 cubic microns, a decrease of

Table 17. Effect of Repeated Hemorrhage on Values for Mean Corpuscular Volume, in Cubic Microns, and Red Cell Diameter, in Microns

\begin{tabular}{lccccc}
\hline \hline \multirow{2}{*}{$\begin{array}{l}\text { Dog } \\
\text { No. }\end{array}$} & \multicolumn{2}{c}{ Mean corpuscular volume } & & \multicolumn{2}{c}{ Red cell diameters } \\
\cline { 2 - 3 } \cline { 5 - 6 } & Normal & $\begin{array}{c}\text { After repeated } \\
\text { hemorrhages }\end{array}$ & & Normal & $\begin{array}{c}\text { After repeated } \\
\text { hemorrhages }\end{array}$ \\
\hline Males & & & & \\
36 & 76.6 & 49.5 & 7.21 & 5.29 \\
37 & 72.1 & 52.5 & 6.95 & 5.59 \\
38 & 75.0 & 56.4 & 7.00 & 5.28 \\
\cline { 6 - 7 } Mean & 74.6 & 52.8 & 7.05 & 5.39 \\
\hline
\end{tabular}

15 per cent, contrasted with a 9 per cent decrease in cell diameter. During the second six-week portion of the post-bleeding period, no further decrease was noted, the value observed at the end of the twelfth post-bleeding week being 57.0 cubic microns. On a percentage basis, therefore, there was a greater decrease in mean corpuscular volume than in cell diameter. This was further substantiated by the results of the study on the three male animals that had been subjected to four or five bleedings each and then observed for approximately four months after bleeding (table 17). One dog, number 36 , displayed a 35 per cent reduction in mean corpuscular volume, from a normal of 76.6 cubic microns to 49.5 cubic microns, and a 26 per cent reduction in cell diameter, from a normal of 7.21 microns to 5.29 microns. A second. animal, number 37 , showed a 27 per cent reduction in mean corpuscular volume, from a normal of 72.1 cubic microns to 52.5 cubic microns, with a 20 per cent reduction in cell diameter, from a normal of 6.95 microns to 5.59 microns. The third dog, number 38 , showed a 25 per cent reduction in mean corpuscular volume, from a normal of 75.0 cubic microns to 56.4 cubic microns, with a 24 per cent reduction in cell 
diameter, from a normal of 7.00 microns to 5.28 microns. The mean decrease in mean corpuscular volume was 29 per cent, and the mean decrease in cell diameter was 24 per cent.

\section{TOTAL CELL VOLUME}

A mean pre-bleeding total cell volume of $574.7 \mathrm{cc}$. was observed for the four animals used in the short-term study (table 18). A mean cell volume of $149.4 \mathrm{cc}$. was removed through bleeding and post-bleeding sampling, which left a predicted mean cell volume of 425.3 cubic centimeters. The actual mean cell volume, observed within 30 minutes of the start of bleeding, was 447.3 cc., a difference of 22.0 cubic centimeters. Sampling for analysis resulted in the removal of an additional volume of $25.4 \mathrm{cc}$. of cells before blood-volume determinations were made 24 hours after hemorrhage. Deducting this quantity from the actual value for mean cell volume after bleeding, 447.3 cc., would leave a net pre-

Table 18. Actual and Predicted Total Cell Volume, in Cubic Centimeters, 30 Minutes and 24 Hours After Bleeding

(Short-term study)

\begin{tabular}{|c|c|c|c|c|c|c|c|}
\hline \multirow[b]{2}{*}{$\begin{array}{l}\text { Dog } \\
\text { No. }\end{array}$} & \multirow{2}{*}{$\begin{array}{l}\text { Last } \\
\text { pre- } \\
\text { bleed- } \\
\text { ing }\end{array}$} & \multirow{2}{*}{$\begin{array}{l}\text { Cell volume } \\
\text { removed in } \\
\text { bleeding and } \\
\text { sampling }\end{array}$} & \multicolumn{2}{|c|}{$\begin{array}{l}\text { Within } 30 \text { minutes } \\
\text { of start of bleeding }\end{array}$} & \multirow{2}{*}{$\begin{array}{l}\text { Cell } \\
\text { volume } \\
\text { removed in } \\
\text { sampling }\end{array}$} & \multicolumn{2}{|c|}{$\begin{array}{l}24 \text { hours } \\
\text { after bleeding }\end{array}$} \\
\hline & & & $\begin{array}{l}\text { Predicted } \\
\text { cell vol. }\end{array}$ & $\begin{array}{l}\text { Actual } \\
\text { cell vol. }\end{array}$ & & $\begin{array}{l}\text { Predicted } \\
\text { cell vol. }\end{array}$ & $\begin{array}{l}\text { Actual } \\
\text { cell vol. }\end{array}$ \\
\hline \multicolumn{8}{|l|}{ Males } \\
\hline \multirow[t]{2}{*}{44} & 669.2 & 231.5 & 437.7 & 472.9 & 26.9 & 446.0 & 484.1 \\
\hline & 603.7 & 129.0 & 474.7 & 448.6 & 24.2 & 424.4 & 470.5 \\
\hline 45 & 672.3 & 147.1 & 525.2 & 475.2 & 29.0 & 446.2 & 564.2 \\
\hline \multirow[t]{2}{*}{46} & 605.0 & 141.8 & 463.2 & 562.1 & 23.3 & 538.8 & 437.2 \\
\hline & 569.9 & 181.7 & 388.2 & 522.2 & 25.8 & 496.4 & 353.7 \\
\hline \multirow[t]{4}{*}{47} & 649.4 & 136.6 & 512.8 & 495.1 & 24.8 & 470.3 & 452.2 \\
\hline & 537.1 & 139.0 & 398.1 & 414.2 & 23.3 & 390.9 & 338.9 \\
\hline & 452.6 & 120.6 & 332.0 & 311.0 & 26.6 & 284.4 & 279.6 \\
\hline & 413.0 & 117.3 & 295.7 & 324.4 & 24.8 & 299.6 & 358.2 \\
\hline Mean & 574.7 & 149.4 & 425.3 & 447.3 & 25.4 & 421.9 & 415.4 \\
\hline
\end{tabular}

dicted mean cell volume of 421.9 cubic centimeters. Actually, the mean cell volume observed 24 hours after hemorrhage was 415.4 cubic centimeters. Therefore, the observed values approximated the predicted values to a surprising degree.

- In terms of body weight, the pre-bleeding mean cell volume of the animals used for the short-term study was $30.5 \mathrm{cc}$. per kilogram (table 19). Again it should be pointed out that this value does not represent the normal mean cell volume for these animals, since gradual decreases in this factor occurred throughout the study. Within 30 minutes after the start of hemorrhage, the observed mean level was $23.2 \mathrm{cc}$. per kilogram, and on the day following bleeding, a value of $21.8 \mathrm{cc}$. per kilogram 
Table 19. Values for Cell Volume, in Cubic Centimeters per Kilogram (Short-term study)

\begin{tabular}{|c|c|c|c|c|c|c|c|}
\hline \multirow{2}{*}{$\begin{array}{l}\text { Dog } \\
\text { No. }\end{array}$} & & \multirow{2}{*}{$\begin{array}{l}\text { Last pre- } \\
\text { bleeeding }\end{array}$} & \multirow{2}{*}{$\begin{array}{l}\text { Within } \\
30 \text { minutes } \\
\text { of start of } \\
\text { bleeding }\end{array}$} & \multicolumn{4}{|c|}{ Post-bleeding period } \\
\hline & & & & 1 day & 2 days & 3 days & 5-7 days \\
\hline \multicolumn{8}{|l|}{ Males } \\
\hline \multirow[t]{2}{*}{44} & & 29.2 & 20.6 & 21.1 & 18.6 & 16.3 & 16.8 \\
\hline & & 26.4 & 19.6 & 20.5 & 20.1 & 17.1 & 19.4 \\
\hline \multirow[t]{2}{*}{45} & & 38.6 & $\ldots \ldots . .$. & 25.7 & 25.9 & 23.5 & 22.7 \\
\hline & & 36.0 & 25.6 & 30.2 & 22.8 & 23.6 & 21.3 \\
\hline \multirow[t]{2}{*}{46} & & 34.2 & 31.8 & 24.8 & 22.4 & 22.2 & 21.8 \\
\hline & & 32.2 & 29.5 & 20.0 & 20.9 & 17.9 & 15.3 \\
\hline \multirow[t]{4}{*}{47} & & 34.4 & 26.2 & 23.9 & 19.2 & 18.3 & 23.0 \\
\hline & & 28.4 & 21.9 & 17.9 & 18.7 & 17.7 & 18.3 \\
\hline & & 24.0 & 16.5 & 14.8 & 14.5 & 15.3 & 12.7 \\
\hline & . & 21.8 & 17.2 & 19.0 & 15.6 & 16.0 & 15.4 \\
\hline Mean & & 30.5 & 23.2 & 21.8 & 19.9 & 18.8 & 18.7 \\
\hline
\end{tabular}

was obtained. On succeeding days further small decreases followed the removal of cells through sampling for analysis, resulting in a mean value of 18.7 cc. per kilogram five to seven days after bleeding.

In the long-term study, the mean normal cell volume for the five female dogs was $32.1 \mathrm{cc}$. per kilogram (table 20). One week after the removal of approximately one half the total blood volume, the mean cell volume was 20.7 cc. per kilogram. Recovery throughout the postbleeding period was slow, attaining a mean cell volume of $22.4 \mathrm{cc}$. per kilogram at the end of the sixth week. For the three dogs that were observed for 12 weeks, a mean cell volume of $19.8 \mathrm{cc}$. per kilogram was noted at the end of the twelfth week, as compared with a mean of 19.3

Table 20. Values for Cell Volume, in Cubic Centimeters per Kilogram (Long-term study)

\begin{tabular}{|c|c|c|c|c|c|c|c|c|c|}
\hline \multirow{2}{*}{$\begin{array}{l}\text { Dog } \\
\text { No. }\end{array}$} & \multirow{2}{*}{$\begin{array}{c}\text { Pre- } \\
\text { bleeding } \\
\text { normal }\end{array}$} & \multicolumn{8}{|c|}{ Post-bleeding period } \\
\hline & & 1-2 days & 1 wk. & 2 wks. & 3 wks. & 4 wks. & 5 wks. & 6 wks. & 12 wks. \\
\hline \multicolumn{10}{|c|}{ Females } \\
\hline 20 & 27.2 & 19.3 & 19.7 & 14.6 & 20.4 & 19.8 & 17.8 & 18.9 & 21.5 \\
\hline 21 & 29.5 & 22.1 & 25.2 & 24.0 & 23.1 & 26.8 & 23.9 & 25.4 & .............. \\
\hline 22 & 28.1 & 20.8 & 17.6 & 22.3 & 21.9 & 22.8 & 26.9 & 28.7 & 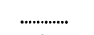 \\
\hline 19 & 41.3 & 22.7 & 22.1 & 22.0 & $\ldots \cdots \cdots \cdots$ & 21.8 & 19.6 & 18.6 & 16.5 \\
\hline 32 & 34.2 & 19.1 & 18.7 & 19.5 & 19.0 & 21.3 & 19.9 & 20.4 & 21.5 \\
\hline Mean & 32.1 & 20.8 & 20.7 & 20.5 & 21.1 & 22.5 & 21.6 & 22.4 & 19.8 \\
\hline \multicolumn{10}{|l|}{ Males } \\
\hline 17 & 36.7 & 24.4 & 22.6 & 22.9 & 29.0 & 23.9 & 22.8 & 24.4 & 20.6 \\
\hline 18 & 26.9 & 16.4 & 16.6 & 17.8 & 19.2 & 22.1 & 19.3 & 18.1 & 20.1 \\
\hline 30 & 29.4 & 22.5 & 24.3 & 29.3 & 31.6 & 32.1 & 38.8 & 33.5 & 34.6 \\
\hline 31 & 30.4 & 23.6 & 24.6 & 27.6 & 28.9 & 31.7 & 34.9 & 30.7 & 32.5 \\
\hline Mean & 30.8 & 21.7 & 22.0 & 24.4 & 27.2 & 27.4 & 28.9 & $26.7^{\circ}$ & 27.0 \\
\hline
\end{tabular}


cc. at the end of the sixth week. It is evident that the volume of cells removed in weekly sampling tended to offset any cell volume regeneration.

For the male animals, the pre-bleeding normal cell volume was 30.8 cc. per kilogram. At the end of the first week following hemorrhage, the volume was $22.0 \mathrm{cc}$. per kilogram. During the post-bleeding period, a gradual increase in cell volume per unit of body weight was noted in these animals. A mean level of $26.7 \mathrm{cc}$. was observed at the end of the sixth week and a mean level of $27.0 \mathrm{cc}$. per kilogram was noted at the end of the twelfth week. Apparently, therefore, the male dogs were capable of a somewhat more satisfactory response than the females. However, since recovery was not complete, even after 12 weeks, it would appear that in the male dogs also weekly sampling played an important part in limiting the degree of recovery.

In adjusting the food level to maintain a fairly constant body. weight, some dogs received more food per kilogram than others. Accordingly, to ascertain whether food intake was a factor in the superior performance of the male animals, the amount of food consumed in the first six weeks of the post-bleeding period was calculated in terms of body weight. For the males, it was found to be $471.9 \mathrm{~g}$. per kilogram; for the females, $539.0 \mathrm{~g}$. (table 21 ). The more satisfactory response of the males, therefore, cannot be attributed to a greater food intake per kilogram of body weight.

Table 21. Amount of Synthetic Ration Consumed, in Grams per Kilogram (Six-Week Period)

(Long-term study)

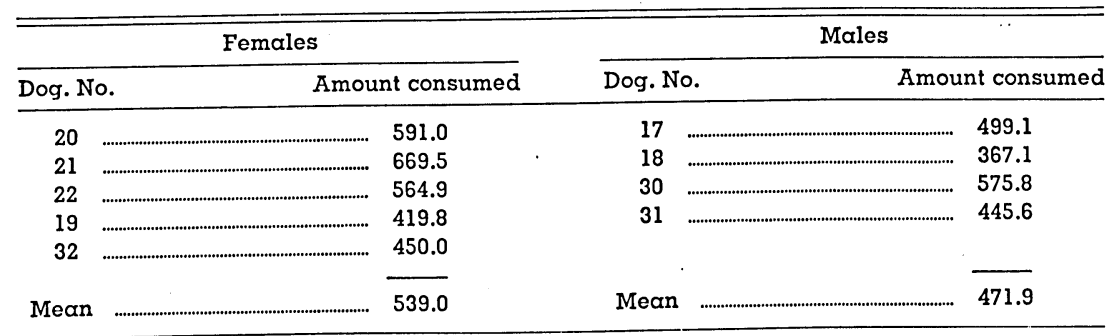

Since the recovery of the male dogs was appreciably better than that of the female dogs during the first six weeks of the post-bleeding period, and since, too, the mean weight of the females was somewhat less than that of the males $(15.7 \mathrm{~kg}$. and $18.8 \mathrm{~kg}$., respectively), it seemed advisable to ascertain whether the slower recovery of the females could be ascribed to the fact that a larger percentage of total cell volume was removed from the females than from the males in the course of bleeding and weekly sampling. Accordingly, the total volume of cells removed during bleeding and weekly sampling in the first six weeks of the postbleeding period was calculated for both males and females and expressed 
Table 22. Percentage of Total Cell Volume Removed Through Bleeding and Weekly Sampling (Six-Week Period)

(Long-term study)

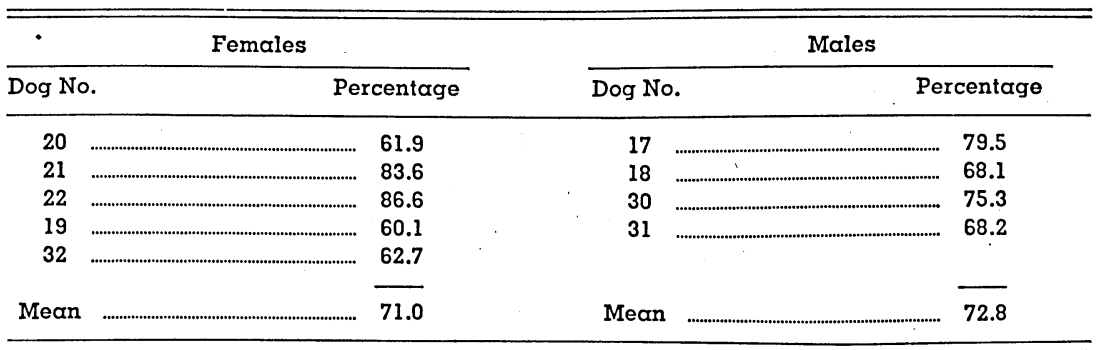

in terms of percentage of original total cell volume. For the males, the mean was found to be 72.8 per cent; for the females, 71.0 per cent (table 22). From these figures, it is evident that the females' retarded recovery cannot be attributed to the loss of a relatively greater quantity of blood cells.

The volume of cells regenerated during the first six weeks of the post-bleeding period, per kilogram of body weight, was also calculated for each dog, resulting in a mean cell volume regeneration of $13.0 \mathrm{cc}$. per kilogram for the females, as against a mean of $18.8 \mathrm{cc}$. per kilogram for the males (table 23).

There was some variation from animal to animal in the percentage of total cell volume removed (table 22). Moreover, wide variations in the volume of cells regenerated were evident within the groups (table 23). Therefore, as a search for an explanation of the observed differences in response, a correlation was run between the percentage of cells removed and cell volume regenerated during the first six weeks of the post-bleeding period. Although the correlation coefficient obtained with this series, +.8128 , was not significant, owing to the limited number of dogs involved, a similar calculation, based on data secured in this investigation plus unpublished data covering other experiments per-

Table 23. Cell Volume Regenerated, in Cubic Centimeters per Kilogram (Six-Week Period)

(Long-term study)

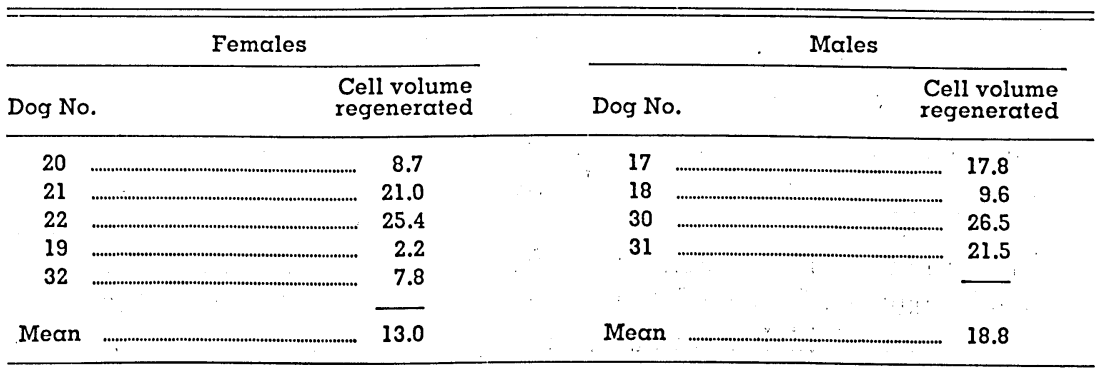


formed in this laboratory on an additional group of dogs, resulted in a significant positive correlation, +.6152 , indicating that one condition that influenced cell volume regeneration was the degree of physiological stress induced by bleeding.

The ranges observed in cell volume regeneration, 2.2 to 25.4 cc. per kilogram for the females, and 9.6 to $26.5 \mathrm{cc}$. per kilogram for the males, suggested that another factor, in addition to physiological stress, was decidedly important in controlling the volume of cells regenerated following hemorrhage. The most obvious explanation was a reserve of blood-building materials in the animal body at the time of hemorrhage. Assuming that such a reserve existed, and in order to discover the extent to which it was a determining influence, the first two or three weeks following the initial bleeding were set up as a "depletion period" during which the reserves in the animal body might be utilized for the regeneration of cells. This interval apparently allowed for depletion of any

Table 24. Cell Volume Regeneration in Cubic Centimeters per Kilogram per Week (Long-term study)

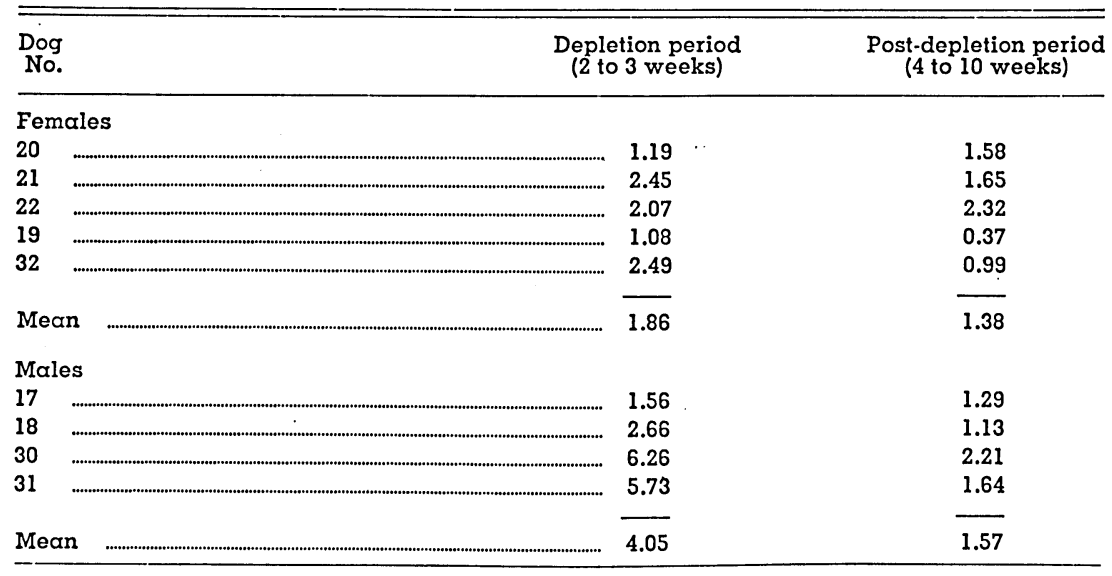

reserves since cell volume regeneration during the remainder of the post-bleeding period continued at a reduced but fairly constant rate. The weekly volume of cells regenerated during the depletion period was calculated for each animal on the basis of body weight, as was the volume of cells regenerated during the "post-depletion period," and these data are presented in table 24.

It was evident during the depletion period that the males were capable of appreciably better cell volume regeneration than the females. Although the marked difference between the means for the males and females (4.05 cc. per kilogram per week for the males as against 1.86 cc. for the females) was attributable in a large measure to the outstanding performance of two of the males, nevertheless three of the male animals produced higher values than any of the females, and the male 
that showed the least regeneration was definitely higher than the lowest females. When Student's " $t$ " test was applied to these data, the difference in the performance of the males and females just fell short of being significant $(\mathrm{P}=<0.086)$. During the post-depletion period, however, the males' mean cell volume regeneration, $1.57 \mathrm{cc}$. per kilogram per week, was only slightly better than that of the females, 1.38 cc. per kilogram per week.

Table 24 also shows individual differences within both groups of animals in their ability to regenerate cells. For the females, cell volume regeneration ranged, during the depletion period, from 1.08 to $2.49 \mathrm{cc}$. per kilogram per week, whereas for the males it ranged from 1.56 to $6.26 \mathrm{cc}$. per kilogram per week. During the post-depletion period, the range for the females was from 0.37 to $2.32 \mathrm{cc}$, and for the males, from 1.13 to $2.21 \mathrm{cc}$. per kilogram per week.

It seemed possible that the amount of food consumed might offer an explanation for these individual differences in the rate of cell volume regeneration during the post-depletion period. It will be recalled that in adjusting the food level to maintain a fairly constant body weight, some animals received more food per kilogram than others. Accordingly, to ascertain to what extent food intake might be a factor in the higher cell volume regeneration level of some animals, the amount of food consumed per kilogram per week was calculated for each of the animals and a correlation run between the amount of food consumed and the volume of cells regenerated per kilogram per week. The correlation coefficient obtained was significant, +.7873 , and demonstrated that cell volume regeneration was at least in part controlled by the amount of food intake. It should be reiterated, however, that this does not explain the difference between the males and females since the females actually consumed more food per kilogram than did the males.

With the thought that the normal cell volume, in cubic centimeters per kilogram, might be one criterion of the blood-building reserves in the animal body, another correlation was run between normal cell volume and the volume of cells regenerated in cubic centimeters per kilogram during the first six weeks of the post-bleeding period. The result was an insignificant correlation coefficient, indicating that no relationship existed between these two factors. It is apparent that the normal cell volume is no indication of the blood-building reserves in the animal body, or at least it is no index of the hemopoietic response which will follow the loss of a considerable amount of blood.

\section{CELL VOLUME PER CENT}

The rapidity with which interstitial fluid is drawn into the blood stream in an effort to restore blood volume following the loss of a significant amount of blood is of vital interest. 
Table 25. Values for Cell Volume Per Cent (Short-term study)

\begin{tabular}{|c|c|c|c|c|c|c|}
\hline \multirow{2}{*}{$\begin{array}{l}\text { Dog } \\
\text { No. }\end{array}$} & \multirow{2}{*}{$\begin{array}{l}\text { Last pre- } \\
\text { bleeding }\end{array}$} & \multirow{2}{*}{$\begin{array}{l}\text { Within } \\
30 \text { minutes } \\
\text { of start of } \\
\text { bleeding }\end{array}$} & \multicolumn{4}{|c|}{ Post-bleeding period } \\
\hline & & & I day & 2 days & 3 days & 5-7 days \\
\hline \multicolumn{7}{|l|}{ Males } \\
\hline \multirow[t]{2}{*}{44} & 38.6 & 33.0 & 31.2 & 28.4 & 26.0 & 26.8 \\
\hline & 35.2 & 34.5 & 31.2 & 30.7 & 26.9 & 28.7 \\
\hline 45 & 46.5 & 43.8 & 41.2 & 35.1 & 35.5 & 48.5 \\
\hline \multirow[t]{2}{*}{46} & 42.1 & 43.0 & 32.1 & 31.0 & 29.1 & 30.7 \\
\hline & 39.9 & 37.8 & 30.7 & 29.9 & 26.1 & 23.0 \\
\hline \multirow[t]{4}{*}{47} & 46.8 & 39.3 & 36.1 & 33.8 & 32.8 & 29.1 \\
\hline & 38.3 & 33.6 & 28.3 & 27.8 & 26.3 & 26.9 \\
\hline & 33.7 & 29.6 & 25.4 & 24.8 & $\cdot 26.0$ & 22.1 \\
\hline & 32.2 & 29.7 & 27.1 & 24.5 & 24.1 & 23.5 \\
\hline Mean & 39.2 & 36.0 & 31.4 & 29.5 & 28.1 & 28.7 \\
\hline
\end{tabular}

Table 26. Values for Cell Volume Per Cent (Long-term study)

\begin{tabular}{|c|c|c|c|c|c|c|c|c|c|}
\hline \multirow{2}{*}{$\begin{array}{l}\text { Dog } \\
\text { No. }\end{array}$} & \multirow{2}{*}{$\begin{array}{c}\text { Pre- } \\
\text { bleeding } \\
\text { normal }\end{array}$} & \multicolumn{8}{|c|}{ Post-bleeding period } \\
\hline & & $1-2$ days & l wk. & 2 wks. & 3 wks. & 4 wks. & 5 wks. & 6 wks. & 12 wks. \\
\hline \multicolumn{10}{|c|}{ Females } \\
\hline 20 & 39.7 & 32.2 & 35.1 & 26.7 & 30.7 & 33.3 & 30.1 & 29.5 & 32.6 \\
\hline 21 & 44.8 & 31.9 & 39.6 & 36.2 & 42.6 & 43.0 & 39.3 & 40.1 & .................. \\
\hline 22 & 45.4 & 34.9 & 34.7 & 38.2 & 37.3 & 40.0 & 44.1 & 47.3 & ............... \\
\hline 19 & 58.8 & 35.2 & 39.3 & 39.1 & $\ldots \ldots \ldots \ldots$ & 36.6 & 37.2 & 36.7 & 35.1 \\
\hline 32 & 49.6 & 33.8 & 31.1 & 33.8 & 33.6 & 36.2 & 34.9 & 36.1 & 37.5 \\
\hline Mean & 47.6 & 33.6 & 35.9 & 34.8 & 36.0 & 37.8 & 37.1 & 37.9 & 35.1 \\
\hline \multicolumn{10}{|l|}{ Males } \\
\hline 17 & 46.8 & 40.8 & 35.4 & 35.5 & 42.1 & 38.7 & 39.0 & 37.4 & 35.0 \\
\hline 18 & 46.1 & 30.6 & 33.3 & 35.5 & 33.8 & 36.6 & 35.7 & 34.1 & 35.8 \\
\hline 30 & 39.0 & 31.2 & 33.2 & 37.6 & 40.0 & 42.6 & 45.4 & 43.0 & 46.3 \\
\hline 31 & 42.6 & 32.9 & 35.5 & 38.9 & 40.9 & 44.1 & 46.3 & 43.2 & 46.1 \\
\hline Mean & 43.6 & 33.9 & 34.4 & 36.9 & 39.2 & 40.5 & 41.6 & 39.4 & 40.8 \\
\hline
\end{tabular}

In the short-term study, it was observed that the mean pre-bleeding cell volume per cent in nine ${ }^{2}$ experiments was 39.2 per cent (table 25 ). Analyses made within 30 minutes of the start of hemorrhage returned a mean cell volume per cent of 36.0 , indicating that within this relatively short period an appreciable amount of dilution had occurred. Complete adjustment followed within the next 24 hours. On the day after bleeding, the observed mean cell volume per cent was 31.4 per cent; on the second day, 29.5 per cent; on the third day, 28.1 per cent. These later decreases resulted from the loss of cells in daily sampling and were un-

2 One experiment, on dog number 45 , was omitted since no value was obtained within 30 minutes of the start of hemorrhage. 
doubtedly also influenced by the alterations noted in mean corpuscular volume.

In the long-term study, the mean pre-bleeding cell volume per cent for the females was 47.6 per cent (table 26). One week after hemorrhage the mean was 35.9 per cent, and at the end of six weeks, 37.9 per cent, showing little recovery. For the three females observed for 12 weeks, the mean at the end of six weeks was 34.1 per cent; at the end of 12 weeks, 35.1 per cent, indicating little improvement in the second sixweek interval.

For the males, the mean pre-bleeding normal cell volume per cent was 43.6 per cent. At the end of the first post-bleeding week the mean was 34.4 per cent; at the end of six weeks, 39.4 per cent; and at the end of 12 weeks, 40.8 per cent. In cell volume per cent, as in cell count and cell volume regeneration, the males responded more satisfactorily than the females, but, just as with the females, they displayed little improvement during the second six-week portion of the post-bleeding period.

\section{INTERRELATIONSHIPS BETWEEN COUNT, SIZE, AND VOLUME OF CELLS}

In the long-term study, the coefficient of correlation obtained between cell count and cell cliameter (-.7745) was significant, indicating a close relationship between cell diameter and the number of cells regenerated in hemorrhagic anemia.

Between cell diameter and mean corpuscular volume another significant coefficient of correlation was obtained $(+.4411)$. Alterations in the other dimensions of the cell, which are not directly proportional to the change in cliameter, probably explain why this correlation was no higher. Although in certain types of anemia there have been observed cells of unusual shapes which would result in an altered relationship between the volume of the erythrocyte and its diameter, cells abnormal in contour were not found, even in severely anemic dogs, in the present study. The correlation coefficient obtained between cell count and cell volume in cubic centimeters per kilogram $(+.3524)$, while significant, indicates that cell count is not an entirely satisfactory measure of total cell volume. No relationship was observed between cell diameter and cell volume in cubic centimeters per kilogram.

\section{OXYGEN-COMBINING CAPACITY}

The oxygen-combining capacity of the blood is usually accepted as the most significant measure of the degree of anemia.

In the short-term study, the mean pre-bleeding oxygen capacity for the 10 experiments was found to be 15.0 volumes per cent (table 27). The decrease observed within 30 minutes after the start of hemorrhage, to 14.4 volumes per cent, was not so great as might have been anticipated 
Table 27. Values for Oxygen-combining Capacity, in Volumes Per Cent (Short-term study)

\begin{tabular}{|c|c|c|c|c|c|c|}
\hline \multirow{2}{*}{$\begin{array}{l}\text { Dog } \\
\text { No. }\end{array}$} & \multirow{2}{*}{$\begin{array}{l}\text { Last pre- } \\
\text { bleeding }\end{array}$} & \multirow{2}{*}{$\begin{array}{l}\text { Within } \\
30 \text { minutes } \\
\text { of start of } \\
\text { bleeding }\end{array}$} & \multicolumn{4}{|c|}{ Post-bleeding period } \\
\hline & & & $1 \mathrm{daY}$ & 2 days & 3 days & 5-7 days \\
\hline $\begin{array}{l}\text { Males } \\
44\end{array}$ & $\begin{array}{l}15.6 \\
14.0\end{array}$ & $\begin{array}{l}13.9 \\
14.8\end{array}$ & $\begin{array}{l}11.9 \\
13.2\end{array}$ & $\begin{array}{l}10.9 \\
12.2\end{array}$ & $\begin{array}{l}10.8 \\
10.7\end{array}$ & $\begin{array}{l}10.7 \\
11.3\end{array}$ \\
\hline 45 & $\begin{array}{l}18.8 \\
16.8\end{array}$ & $\begin{array}{l}18.5 \\
17.7\end{array}$ & $\begin{array}{l}14.7 \\
14.0\end{array}$ & $\begin{array}{l}16.6 \\
12.6\end{array}$ & $\begin{array}{l}14.7 \\
13.6\end{array}$ & $\begin{array}{l}14.6 \\
11.6\end{array}$ \\
\hline 46 & $\begin{array}{l}17.3 \\
15.0\end{array}$ & $\begin{array}{l}16.0 \\
13.6\end{array}$ & $\begin{array}{l}13.4 \\
11.3\end{array}$ & $\begin{array}{l}13.0 \\
13.1\end{array}$ & $\begin{array}{l}12.5 \\
10.6\end{array}$ & $\begin{array}{r}12.3 \\
9.1\end{array}$ \\
\hline 47 & $\begin{array}{l}14.0 \\
13.3 \\
13.0 \\
12.4\end{array}$ & $\begin{array}{l}11.8 \\
13.4 \\
11.5 \\
12.3\end{array}$ & $\begin{array}{r}12.3 \\
11.0 \\
10.2 \\
9.6\end{array}$ & $\begin{array}{l}13.4 \\
11.3 \\
10.0 \\
10.1\end{array}$ & $\begin{array}{r}12.6 \\
11.1 \\
8.0 \\
9.1\end{array}$ & $\begin{array}{r}11.2 \\
6.5 \\
8.1 \\
8.3\end{array}$ \\
\hline Mean & 15.0 & 14.4 & 12.2 & 12.3 & 11.4 & 10.4 \\
\hline
\end{tabular}

from the change in cell volume per cent (39.2 to 36.0 per cent). Since the sample used for determining oxygen capacity was always secured before the determination of cell volume per cent, it is possible that the time elapsing between the securing of these two samples might have accounted for the lack of agreement. Unquestionably this was a period during which there was an exceedingly rapid infiltration of cellular fluid into the blood stream.

On the first day after hemorrhage, the mean oxygen capacity observed was 12.2 volumes per cent. It is interesting to note the close agreement between the decrease in cell volume per cent (19.9 per cent) and the decrease in oxygen capacity (18.7 per cent), although the agreement between these two factors was not quite so close on the second and third days after hemorrhage. For example, by the third day after bleeding, the observed decrease in cell volume per cent was 28.3 per cent as contrasted with a decrease in oxygen capacity of 24.0 per cent. Nevertheless, it would seem that these values are as close as could be expected and are not indicative of alterations in the hemoglobin concentration in the cell.

In the long-term study, the pre-bleeding normal oxygen capacity for the females was 18.0 volumes per cent (table 28). At the end of the first week, following loss of approximately one half of the determined blood volume, the mean oxygen capacity was 12.6 volumes per cent. A further slight decrease occurred during the first six weeks of the post-bleeding period, the oxygen capacity at the end of the sixth week being 11.4 volumes per cent. For the three females observed for 12 weeks, the mean at the end of the twelfth week was 11.8 volumes per cent as compared with 11.7 volumes per cent for these same dogs at the end of the sixth week. Evidently the weekly loss of from 30 to 50 cc. of blood through sampling offset any recovery that was made. 
Table 28. Values for Oxygen-combining Capacity, in Volumes Per Cent (Long-term study)

\begin{tabular}{|c|c|c|c|c|c|c|c|c|c|}
\hline \multirow{2}{*}{$\begin{array}{l}\text { Dog } \\
\text { No. }\end{array}$} & \multirow{2}{*}{$\begin{array}{c}\text { Pre- } \\
\text { bleeding } \\
\text { normal }\end{array}$} & \multicolumn{8}{|c|}{ Post-bleeding period } \\
\hline & & 1-2 days & 1 wk. & 2 wks. & 3 wks. & 4 wks. & 5 wks. & 6 wks. & 12 wks. \\
\hline \multicolumn{10}{|c|}{ Females } \\
\hline 20 & 14.2 & 9.8 & 12.7 & 11.5 & 9.1 & 9.5 & 7.1 & 10.7 & 10.7 \\
\hline 21 & 14.7 & 11.6 & 13.5 & 11.3 & 12.2 & 12.0 & 11.7 & 10.8 & $\ldots \ldots \ldots . . .$. \\
\hline 22 & 18.5 & 10.4 & 9.2 & 9.9 & 8.8 & 10.7 & 11.6 & 11.0 & $\ldots \ldots \ldots \ldots . .$. \\
\hline 19 & 22.8 & 16.0 & 16.4 & 14.5 & 15.0 & 15.2 & 13.8 & 12.2 & 10.8 \\
\hline 32 & 19.7 & 11.8 & 11.1 & 11.0 & 11.1 & 11.9 & 12.3 & 12.3 & 13.8 \\
\hline Mean & 18.0 & 11.9 & 12.6 & 11.6 & 11.2 & 11.9 & 11.3 & 11.4 & 11.8 \\
\hline \multicolumn{10}{|l|}{ Males } \\
\hline 17 & 18.9 & 13.9 & 13.5 & 14.6 & 13.5 & 15.1 & 14.7 & 14.0 & 11.3 \\
\hline $18{ }^{\circ}$ & 17.3 & 12.7 & $\ldots \ldots \ldots \ldots . .$. & 10.4 & 11.0 & 10.5 & 11.6 & 11.4 & 10.5 \\
\hline 30 & 17.3 & 10.5 & 13.0 & 13.2 & 12.9 & 15.6 & 15.7 & 14.8 & 16.9 \\
\hline 31 & 18.7 & 11.9 & 11.4 & 12.6 & 13.4 & 14.0 & 16.9 & 15.9 & 15.2 \\
\hline Mean & 18.0 & 12.2 & 12.6 & 12.7 & 12.7 & 13.8 & 14.7 & 14.0 & 13.5 \\
\hline
\end{tabular}

For the males, the pre-bleeding oxygen capacity was 18.0 volumes per cent; at the end of the first week of the post-bleeding period it was 12.6 volumes per cent. Attention is called to the fact that these values are identical with those reported for the females. The means for oxygen capacity for the males, observed at the end of six weeks and 12 weeks, were 14.0 and 13.5 volumes per cent, respectively. Although these values were somewhat higher than those reported for the females, they were still considerably below the pre-bleeding mean for these animals.

Table 29. Oxygen-combining Capacity-Comparison of Normal Levels with Levels Observed Six Weeks After Hemorrhage

(Long-term study)

\begin{tabular}{|c|c|c|c|}
\hline $\begin{array}{l}\text { Dog } \\
\text { No. }\end{array}$ & $\begin{array}{l}\text { Pre-bleeding } \\
\text { normal in } \\
\text { volumes } \\
\text { per cent } \\
\end{array}$ & $\begin{array}{l}\text { At end of sixth } \\
\text { post-bleeding } \\
\text { week in } \\
\text { volumes per cent }\end{array}$ & $\begin{array}{c}\text { Differences } \\
\text { in volumes } \\
\text { per cent }\end{array}$ \\
\hline \multicolumn{4}{|c|}{ Females } \\
\hline $20 \ldots . . .$. & 14.2 & 10.7 & -3.5 \\
\hline 21 & 14.7 & 10.8 & -3.9 \\
\hline 22 & 18.5 & 11.0 & -7.5 \\
\hline 19 & 22.8 & 12.2 & -10.6 \\
\hline 32 & 19.7 & 12.3 & -7.4 \\
\hline Mean & 18.0 & 11.4 & -6.6 \\
\hline \multicolumn{4}{|l|}{ Males } \\
\hline $17 \ldots$ & 18.9 & 14.0 & -4.9 \\
\hline 18 & 17.3 & 11.4 & -5.9 \\
\hline 30 & 17.3 & 14.7 & -2.6 \\
\hline 31 & 18.7 & 15.9 & -2.8 \\
\hline Mean & ......... $\quad 18.0$ & 14.0 & -4.0 \\
\hline
\end{tabular}


Since there were such marked individual differences in the degree of recovery shown by the animals, table 29 was prepared to show the normal oxygen capacity in volumes per cent, the level at the end of the first six weeks of the post-bleeding period, and the difference between the two levels. The smaller the difference, the more closely did the animal approach his pre-bleeding level and attain complete recovery.

An effort was made to ascertain whether the degree of recovery as measured by these differences was related to the normal oxygen capacity of the blood by running a correlation between the two sets of values. The correlation coefficient obtained, +.7358 , was not significant. Again, based on data secured in this investigation plus unpublished data covering other experiments performed in this laboratory on an additional group of dogs, a significant correlation coefficient, +.5458 , was obtained, indicating that the animals with the highest normal levels were the furthest from their own normal levels at the end of the post-bleeding period.

It is generally assumed that $1.34 \mathrm{cc}$. of oxygen is equivalent to 1.0 g. of hemoglobin. Accordingly, this value together with the volume of cells was used as the basis for calculating hemoglobin regeneration

Table 30. Hemoglobin Regeneration in Grams per Kilogram per Week (Long-term study)

\begin{tabular}{|c|c|c|}
\hline $\begin{array}{l}\text { Dog } \\
\text { No. }\end{array}$ & $\begin{array}{l}\text { Depletion period } \\
. \quad \text { (2 to } 3 \text { weeks) }\end{array}$ & $\begin{array}{l}\text { Post-depletion period } \\
\text { (4 to } 10 \text { weeks) }\end{array}$ \\
\hline \multicolumn{3}{|c|}{ Females } \\
\hline $20 \quad \ldots$. & 0.49 & 0.20 \\
\hline 21 & 0.14 & 0.39 \\
\hline 22 & 0.11 & 0.46 \\
\hline 19 & -0.11 & 0.05 \\
\hline 32 & $\quad 0.38$ & 0.42 \\
\hline Mean & 0.20 & 0.30 \\
\hline \multicolumn{3}{|l|}{ Males } \\
\hline $17 \quad \ldots$ & 0.49 & 0.13 \\
\hline 18 & 0.55 & 0.25 \\
\hline 30 & 1.81 & 0.57 \\
\hline 31 & 1.04 & 0.65 \\
\hline Mean & 0.97 & 0.40 \\
\hline
\end{tabular}

during the post-bleeding period. The first two or three weeks following the initial bleeding were set up as a depletion period, as in the case of cell volume regeneration. Hemoglobin regeneration, per kilogram per week, was then calculated for the depletion period and also for the remainder of the post-bleeding period. The results are given in table 30;" which demonstrates that during the depletion period the males showed a considerably greater capacity for regenerating hemoglobin than they did during the post-depletion period, the mean for the depletion 
period being $0.97 \mathrm{~g}$., and for the post-depletion period, $0.40 \mathrm{~g}$. per kilogram per week. This difference failed to be statistically significant when tested by means of the standard error of the difference ( $\mathrm{P}$ $=0.080$ ). The females, on the other hand, failed to show this greater capacity for regeneration during the depletion period, the mean value observed, $0.20 \mathrm{~g}$., being in fact slightly below the mean noted for the post-depletion period, $0.30 \mathrm{~g}$. per kilogram per week, from which it is apparent that any reserve the females may have had must have been exhausted, perhaps even before bleeding, possibly in the sampling necessary for the establishment of the normal levels.

In the depletion period, the male animals were capable of regenerating hemoglobin more rapidly than the females, as evidenced by Student's " $\mathrm{t}$ " test $(\mathrm{P}=0.035)$. During the post-depletion period, however, although the males continued to display some superiority over the females in the regeneration of hemoglobin, the difference between the two groups was not very large.

As with cell volume regeneration, wide individual differences were apparent in the various animals' capacity for hemoglobin regeneration. For the females the range during the depletion period was from -0.11 to $+0.49 \mathrm{~g}$. per kilogram per week, and for the males, from 0.49 to $1.81 \mathrm{~g}$. per kilogram per week. During the post-depletion period the observed range for the females was from 0.05 to $0.46 \mathrm{~g}$. per kilogram per week, and for the males, from 0.13 to $0.65 \mathrm{~g}$. per kilogram per week.

To test whether food intake influenced the degree of hemoglobin regeneration during the post-depletion period, a correlation was run between food intake in grams per kilogram per week and hemoglobin regeneration in grams per kilogram per week. The correlation coefficient obtained, +.4192 , was not significant, indicating the hemoglobin regeneration was not related to the level of food intake, a result contrary to that obtained. for cell volume regeneration.

\section{LEUCOCYTES}

The immediate effect of hemorrhage, as shown in the short-term study, was to decrease slightly the number of leucocytes per cubic millimeter of blood, from a normal of 7.50 thousands to 7.05 thousands (table 31 ). No doubt this decrease, as with the red blood cells, was due to dilution of the blood with interstitial fluid. The mean white cell counts on succeeding days were somewhat above the pre-bleeding level, being 8.13, 8.47, and 7.89 thousands, on the first, second, and third days, respectively, suggesting a mild leucocytosis as a result of hemorrhage. However, calculation of the standard error of the difference showed that the observed increase was not significant.

In the long-term study, there was also some increase in leucocytes, which again proved to be insignificant. For the females, at the end of the first week, the mean count was 10.30 thousands per cubic millimeter 
Table 31. Values for Leucocyte Counts, in Thousands per Cubic Millimeter (Short-term study)

\begin{tabular}{|c|c|c|c|c|c|c|}
\hline \multirow{2}{*}{$\begin{array}{l}\text { Dog } \\
\text { No. }\end{array}$} & \multirow{2}{*}{$\begin{array}{l}\text { Last pre- } \\
\text { bleeding }\end{array}$} & \multirow{2}{*}{$\begin{array}{l}\text { Within } \\
30 \text { minutes } \\
\text { of start of } \\
\text { bleeding }\end{array}$} & \multicolumn{4}{|c|}{ Post-bleeding period } \\
\hline & & & 1 day & 2 days & 3 days & 5-7 days \\
\hline \multicolumn{7}{|l|}{ Males } \\
\hline \multirow[t]{2}{*}{44} & 7.20 & 5.08 & 6.24 & 6.64 & 6.01 & 6.54 \\
\hline & 8.89 & 5.90 & 6.99 & 7.45 & 6.21 & 6.21 \\
\hline \multirow[t]{2}{*}{45} & 4.75 & 3.65 & 5.45 & 5.02 & 6.89 & 5.52 \\
\hline & 5.58 & 5.02 & 6.08 & 7.34 & 6.86 & 6.55 \\
\hline \multirow[t]{2}{*}{46} & 9.44 & 14.04 & 9.54 & 9.39 & 8.34 & 7.78 \\
\hline & 6.78 & 6.06 & 8.72 & 9.59 & 7.59 & 7.79 \\
\hline \multirow[t]{4}{*}{47} & 6.34 & 7.46 & 8.86 & 7.70 & 8.21 & 7.89 \\
\hline & 8.88 & 8.62 & 10.34 & 12.48 & 10.99 & 10.44 \\
\hline & 10.06 & 7.38 & 11.02 & 10.02 & 9.38 & 17.82 \\
\hline & 7.10 & 7.32 & 8.10 & 9.09 & 8.40 & 8.34 \\
\hline Mean & 7.50 & 7.05 & 8.13 & 8.47 & 7.89 & 8.49 \\
\hline
\end{tabular}

of blood, contrasted with a pre-bleeding mean of 5.85 thousands (table 32). By the end of the sixth week, the mean count was still slightly above normal ( 6.57 thousands per cubic millimeter), but by the end of the twelfth week, the count (based on three dogs) had returned to normal. For the males, the pre-bleeding mean was 7.41 thousands per cubic millimeter. By the end of the sixth and twelfth weeks, the mean counts were 7.71 and 7.56 thousands, respectively. Values slightly above the mean normal count were observed one to two days after bleeding ( 8.59 thousands) and again at the end of the second week

Table 32. Values for Leucocyte Counts, in Thousands per Cubic Millimeter (Long-term study)

\begin{tabular}{|c|c|c|c|c|c|c|c|c|c|}
\hline \multirow[b]{2}{*}{$\begin{array}{l}\text { Dog } \\
\text { No. }\end{array}$} & \multirow{2}{*}{$\begin{array}{c}\text { Pre- } \\
\text { bleeding } \\
\text { normal }\end{array}$} & \multicolumn{8}{|c|}{ Post-bleeding period } \\
\hline & & 1-2 days & 1 wk. & 2 wks. & 3 wks. & 4 wks. & 5 wks. & 6 wks. & 12 wks. \\
\hline \multicolumn{10}{|c|}{ Females } \\
\hline 20 & 9.55 & 5.40 & 8.95 & 7.10 & 6.55 & 7.20 & 9.80 & 5.30 & 6.10 \\
\hline 21 & 3.25 & 7.00 & 13.45 & 7.15 & 4.75 & 5.40 & 5.15 & 10.25 & $\ldots . . . \cdots \cdots \cdot . \cdot$ \\
\hline 22 & 4.15 & 10.20 & 15.60 & 13.45 & 9.25 & 7.30 & 8.80 & 4.50 & $\ldots \ldots \ldots$ \\
\hline 19 & 4.55 & 4.15 & 5.55 & 4.50 & 5.15 & 4.45 & 3.60 & 3.90 & 3.35 \\
\hline 32 & 7.75 & 8.40 & 7.95 & 7.70 & 9.00 & 6.95 & 7.45 & 8.90 & 6.75 \\
\hline Mean & 5.85 & 7.03 & 10.30 & 7.98 & 6.94 & 6.26 & 6.96 & 6.57 & 5.40 \\
\hline \multicolumn{10}{|l|}{ Males } \\
\hline 17 & 5.30 & 9.45 & 9.45 & 9.55 & 10.10 & 5.45 & 7.15 & 12.00 & 9.30 \\
\hline 18 & 5.10 & 7.55 & 6.80 & 7.20 & 8.70 & 5.80 & 6.50 & 4.25 & 5.40 \\
\hline 30 & 8.80 & 8.75 & 5.25 & 17.00 & 8.80 & 5.70 & 7.85 & 6.50 & 6.55 \\
\hline 31 & 10.45 & 8.60 & 8.25 & 8.40 & 7.50 & 8.65 & 8.15 & 8.10 & 9.00 \\
\hline Mean & 7.41 & 8.59 & 7.44 & 10.54 & 8.77 & 6.40 & 7.41 & 7.71 & 7.56 \\
\hline
\end{tabular}


(10.54 thousands) and third week (8.77 thousands), with essentially normal values for the other experimental weeks. The mean for the second week (10.54 thousands) was influenced markedly by the high value observed for dog number 30 , although two of the other males were also somewhat above their normal levels at that time.

It seemed possible that more pronounced changes in the total or differential leucocyte count might be found in the dogs subjected to repeated bleedings (third study). These three animals were bled four or five times each, but no increase in the total leucocyte count was noted as a result of repeated hemorrhage (table 33 ).

Table 33. Values for Leucocyte Counts, in Thousands per Cubic Millimeter (Dogs subjected to repeated bleedings)

\begin{tabular}{|c|c|c|}
\hline Dog No. & Pre-bleeding & Post-bleeding \\
\hline \multicolumn{3}{|l|}{ Males } \\
\hline \multirow{5}{*}{$36 \quad \ldots$} & $\begin{array}{l}\ldots \ldots \ldots . . \\
13.2\end{array}$ & 12.0 \\
\hline & 12.0 & 9.9 \\
\hline & 10.0 & 14.5 \\
\hline & 11.5 & 10.2 \\
\hline & 12.6 & 13.7 \\
\hline \multirow[t]{4}{*}{37} & 10.0 & 11.4 \\
\hline & 11.4 & 9.1 \\
\hline & 9.1 & 9.6 \\
\hline & 7.3 & 8.0 \\
\hline \multirow[t]{5}{*}{38} & 8.7 & 11.4 \\
\hline & 11.4 & 11.3 \\
\hline & 11.3 & 13.7 \\
\hline & 11.5 & 10.2 \\
\hline & 14.3 & 13.6 \\
\hline Mean & 11.0 & $\overline{11.3}$ \\
\hline
\end{tabular}

On the basis of the differential leucocyte count there would appear to be a small increase in the young band-form neutrophiles as a result of hemorrhage and a corresponding decrease in total lymphocytes involving both the small and large forms. The percentage of the other forms of leucocytes did not appear to be noticeably altered (table 34).

\section{DISCUSSION}

Anemia of hemorrhagic origin is found often enough in human subjects to make a study of the complete blood picture a matter of considerable importance.

The purpose of this investigation was to secure a more thorough understanding than has been general up to the present time of conditions that exist in the blood of a subject suffering from hemorrhagic anemia. Accordingly, all factors that seemed to offer a clue to the difficulties 
Table 34. Differential Leucocyte Counts (Dogs subjected to repeated bleedings)

\begin{tabular}{|c|c|c|c|c|c|c|c|c|c|c|c|c|c|c|c|c|}
\hline \multirow{3}{*}{$\begin{array}{l}\text { Dog } \\
\text { No. }\end{array}$} & \multicolumn{6}{|c|}{ Percentage of neutrophiles } & \multicolumn{4}{|c|}{ Percentage of lymphocytes } & \multirow{2}{*}{\multicolumn{2}{|c|}{$\begin{array}{l}\text { Percentage of } \\
\text { monocytes }\end{array}$}} & \multirow{2}{*}{\multicolumn{2}{|c|}{$\begin{array}{l}\text { Percentage of } \\
\text { eosinophiles }\end{array}$}} & \multirow{2}{*}{\multicolumn{2}{|c|}{$\begin{array}{l}\text { Percentage of } \\
\text { basophiles }\end{array}$}} \\
\hline & \multicolumn{2}{|c|}{ Immature } & \multicolumn{2}{|c|}{ Young band forms } & \multicolumn{2}{|c|}{ Mature (segmented) } & \multicolumn{2}{|c|}{ Small } & \multicolumn{2}{|c|}{ Large } & & & & & & \\
\hline & $\begin{array}{l}\text { Before } \\
\text { bleed- } \\
\text { ing }\end{array}$ & $\begin{array}{l}\text { After } \\
\text { bleed- } \\
\text { ing }\end{array}$ & $\begin{array}{l}\text { Before } \\
\text { bleed- } \\
\text { ing }\end{array}$ & $\begin{array}{l}\text { After } \\
\text { bleed- } \\
\text { ing }\end{array}$ & $\begin{array}{l}\text { Before } \\
\text { bleed- } \\
\text { ing }\end{array}$ & $\begin{array}{c}\text { After } \\
\text { bleed- } \\
\text { ing }\end{array}$ & $\begin{array}{l}\text { Before } \\
\text { bleed- } \\
\text { ing }\end{array}$ & $\begin{array}{l}\text { After } \\
\text { bleed- } \\
\text { ing }\end{array}$ & $\begin{array}{l}\text { Before } \\
\text { bleed- } \\
\text { ing }\end{array}$ & $\begin{array}{l}\text { After } \\
\text { bleed. } \\
\text { ing }\end{array}$ & $\begin{array}{c}\text { Before } \\
\text { bleed- } \\
\text { ing }\end{array}$ & $\begin{array}{l}\text { After } \\
\text { bleed- } \\
\text { ing }\end{array}$ & $\begin{array}{l}\text { Before } \\
\text { bleed- } \\
\text { ing }\end{array}$ & $\begin{array}{l}\text { After } \\
\text { bleed- } \\
\text { ing }\end{array}$ & $\begin{array}{l}\text { Before } \\
\text { bleed- } \\
\text { ing }\end{array}$ & $\begin{array}{l}\text { After } \\
\text { bleed- } \\
\text { ing }\end{array}$ \\
\hline \multicolumn{17}{|l|}{ Males } \\
\hline \multirow[t]{3}{*}{36} & 0.5 & 0.0 & 17.5 & 18.5 & 53.5 & 58.5 & 13.5 & 12.5 & 4.0 & 5.0 & 2.5 & 1.5 & 8.5 & 4.0 & 0.0 & 0.0 \\
\hline & 0.0 & 4.5 & 25.5 & 25.0 & 53.0 & 40.0 & 14.5 & 20.5 & 2.5 & 1.0 & 1.5 & 1.5 & 2.5 & 5.0 & 0.5 & 2.5 \\
\hline & 1.0 & 0.5 & 11.0 & 12.5 & 65.5 & 67.0 & 11.5 & 8.5 & 1.0 & 0.5 & 1.5 & 1.0 & 8.5 & 10.0 & 0.0 & 0.0 \\
\hline \multirow[t]{2}{*}{37} & 2.0 & 0.5 & 15.5 & 22.0 & 54.0 & 56.5 & 23.0 & 16.0 & 4.0 & 2.5 & 1.5 & 2.5 & 0.0 & 0.0 & 0.0 & 0.0 \\
\hline & 0.5 & 3.0 & 8.5 & 13.5 & 67.5 & 63.5 & 14.0 & 12.0 & 2.0 & 0.5 & 2.5 & 1.0 & 5.0 & 6.5 & 0.0 & 0.0 \\
\hline \multirow[t]{4}{*}{38} & 0.0 & 0.5 & 8.5 & 13.5 & 65.0 & 68.5 & 16.5 & 11.5 & 5.0 & 3.0 & 5.0 & 2.5 & 0.0 & 0.5 & 0.0 & 0.0 \\
\hline & 0.5 & 1.5 & 13.5 & 17.5 & 68.5 & 68.5 & 11.5 & 7.5 & 3.0 & 1.5 & 2.5 & 1.0 & 0.5 & 2.5 & 0.0 & 0.0 \\
\hline & 0.5 & 0.0 & 14.0 & 20.0 & 69.5 & 58.0 & 8.5 & 15.0 & 0.0 & 0.0 & 1.0 & 0.5 & 6.5 & 6.0 & 0.0 & 0.5 \\
\hline & 0.0 & 0.5 & 9.5 & 7.0 & 65.5 & 72.5 & 21.5 & 7.5 & 1.0 & 0.0 & 0.5 & 0.5 & 2.0 & 12.0 & 0.0 & 0.0 \\
\hline Mean & 0.5 & 1.2 & 13.7 & 16.6 & 62.4 & 61.4 & 14.9 & 12.3 & 2.5 & 1.5 & 2.0 & 1.3 & 3.7 & 5.2 & 0.0 & 0.3 \\
\hline
\end{tabular}


involved in recovery following loss of appreciable amounts of blood have been studied simultaneously and will be discussed in the following paragraphs.

\section{Erythrocyte Counts}

It is generally assumed that the animal body has reserve stores of cells, said to be located largely in the spleen, upon which it can draw in an emergency. From the data obtained through the present research, however, it would appear that either such stores were not available in these experimental animals, or, although this seems highly improbable, the losses of blood to which they were subjected were not sufficiently drastic to release these stores. At the same time, it was demonstrated that animals do have varying quantities of cell-building materials which are used during the early stages of recovery for blood regeneration.

It is eviclent from the results of this investigation that red cell counts, oxygen-combining capacity determinations, and cell volume per cent readings alone will not indicate the severity of the anemia that follows acute loss of blood. Nevertheless, these factors must be considered in conjunction with total blood volume determinations in order to obtain a true picture of a subject's condition. This is demonstrated by results obtained in the short-term study. Had the total blood volume been restored to normal entirely through dilution with interstitial fluid, the decrease in red cell count 24 hours after hemorrhage would have been 26 per cent. However, only about two thirds of the loss in blood volume was restored, and the reduction in red cell count was only 20 per cent. Assuming that the restoration of blood volume was wholly in the plasma fraction, this degree of dilution would have resulted in a decrease in red cell count to 5.56 millions, whereas the observed value was 5.46 millions. With these two values in such close agreement, it is apparent that the recovery in total blood volume must have been due entirely to an increase in plasma and not to a replacement of any portion of the lost cells.

In the long-term study, red cell counts returned to normal five to seven weeks after hemorrhage. In the males the normal level had been regained in five weeks; in the females, not until the seventh week. These data are of greater significance in conjunction with those on cell size and total cell volume. Therefore, further discussion will be reserved for inclusion with those factors.

\section{Cell Size}

In recent years cell size has been receiving increasing emphasis as a diagnostic measure, although as early as 1924-25 Haden stated that the extent of the decrease in cell size in chronic hemorrhagic anemia was probably the best measure of the drain on the bone marrow and 
was accordingly of definite progniostic value. Witts (1931) pointed out that in human subjects chronic microcytic anemia is essentially a disease of the female sex and of the reproductive period and its occurrence in men is an indication of blood loss through accidents, gastric hemorrhage, and the like. Wright (1933), Barer, Fowler, and Baldridge (1934-35), and Fowler and Barer (1937) postulated that certain cases of idiopathic microcytic or hypochromic anemia might be associated with relatively large menstrual losses, while Wintrobe (1934) suggested a classification of anemias on the basis of the size and hemoglobin content of the erythrocytes.

In the short-term study, the slight increase in mean corpuscular volume noted within 30 minutes of the start of bleeding may have been due to hyperemia resulting from a lowered colloid osmotic pressure of the plasma brought about by the observed decrease in both plasma protein and lipide phosphorus, or it may have been the result of alterations in the hydrogen-ion concentration of the plasma. Bennett (1926) demonstrated that within 30 minutes after hemorrhage there was a decrease in hydrogen-ion concentration, with an increase to above normal on the day following hemorrhage, and a return to normal a few days later. It has also been shown by other workers (Price-Jones, 1922; Smirk, 1928; Falik and Bielinski, 1931) that alterations in $\mathrm{pH}$ influence the size of cells, which increase in size with increased acidity and decrease with increased alkalinity. The decrease in mean corpuscular volume, noted in the present study on the second day following hemorrhage, would suggest an increased alkalinity of the blood, as reported by Bennett. On the other hand, this decrease in mean corpuscular volume could have resulted from recovery of the colloid osmotic pressure, since, as shown in the sections dealing with these fractions, plasma proteins and lipide phosphorus had in a large measure returned to normal 24 hours after bleeding.

Haden (1930), in reporting his work with human subjects, observed that changes in mean corpuscular volume were greater than changes in cell diameter. The data in the long-term study and on the three animals that were bled four to five times bear out Haden's observation, which is confirmed further by the fact that the correlation coefficient obtained shows no high degree of relationship between these two values during the post-bleeding period.

The extent to which body reserves of blood-building materials are depleted by hemorrhage influences the degree to which the cells are decreased in size, as is shown by the fact that in the study in which the animals were bled four or five times much greater decreases, both in cell diameter and in mean corpuscular volume, were observed than in the animals that were bled only twice, as in the long-term study. This is further borne out by the highly significant correlation coefficient secured between red cell count and cell diameter for the six animals used in the long-term study for which diameter data were available. 
The more rapid the cell production, the smaller the cells produced.

Microcytosis was found in all of the experimental animals, indicating that in chronic blood loss dogs respond in this respect similarly to the way human subjects react (Price-Jones, 1922; Murphy and Fitzhugh, 1930; Haden, 1932; Wintrobe, 1929-30) rather than like rabbits, in which an increased cell diameter was observed (Ponder and Millar, 1928).

In the present investigation, the female animals displayed posthemorrhagic decreases in cell size earlier than did the males, but except for this greater susceptibility on the part of the female animals: there was no essential difference in the character of the response.

\section{Total Cell Volume}

The factors limiting recovery following acute blood loss may be one or both of two kinds. There may be a reduced capacity for restoring or building cells, or there may be a reduced capacity for building hemoglobin. The lack of ability to build cells will be reflected also in the inability to restore the hemoglobin to the normal level. On the other hand, an animal's organism may be capable of restoring cells which in the absence of ability to restore hemoglobin will not be completely saturated with this pigment. In the present investigation an attempt was made to study not only the animals' capacity for building cells, but also their capacity for building hemoglobin.

In determining the volume of cells which an animal can produce following hemorrhage, due consideration should be given to the possible existence of a cell reserve which could be drawn into the blood stream. As pointed out in the section on cell counts, there was no evidence in the data acquired in the short-term study of a cell reserve in the animal body. This was confirmed by the results of observations made on total cell volume in that the mean total cell volume, measured both 30 minutes after the start of bleeding and 24 hours later, showed remarkable agreement with the predicted values based on the difference between the prebleeding normal mean total cell volume and the volume of cells removed in bleeding and sampling. The amount removed in daily sampling more than offset the amount the animals produced; hence there was a gradual decrease in total cell volume throughout the sampling period.

It is possible that inability to substantiate the existence of a reserve of preformed cells may have resulted from errors inherent in the dye method used for determining total cell volume. It has been reported by Smith, Arnold, and Whipple (1921) that the dye method produces values for total cell volume which are somewhat higher than the circulating cell volume in the animal body. This may tend to conceal the fact that reserve stores of cells actually exist. It would, however, necessarily mean that the reserve was essentially of the same magnitude as the error inherent in the dye method. 
Although cell counts were restored to normal during the long-term study, owing to decrease in cell size, cell volume remained definitely below normal throughout the post-bleeding period. Cell count, therefore, apparently is not a dependable measure of the total volume of cells in a subject recovering from loss of blood.

When the post-bleeding period was divided into a "depletion" period representing the first two or three weeks following bleeding, and a "post-depletion" period representing the balance of the post-bleeding interval, evidence was found of the existence of reserves of cell-building materials in the fact that the animals were capable of much greater cell volume regeneration during the first two or three weeks following hemorrhage than they were during the remainder of the post-bleeding period. The fact that the males possessed appreciably greater reserves of cellbuilding materials than the females was shown by their more rapid cell volume regeneration during the depletion period. During the depletion period, the rate of regeneration for the males was $21 / 2$ times as rapid as during the post-depletion period, whereas for the females the rate of regeneration during the depletion period exceeded only by one third the regeneration noted for the post-depletion period. Apparently the reserve had been essentially exhausted, however, at the end of the depletion period because, although they continued to do slightly better than the females during the remainder of the period, the difference was small.

Wide individual differences in cell volume regeneration were apparent, there being animals within each group that apparently had high capacity, as well as some with low capacity, for cell volume regeneration. There is evidence that this was due to some condition inherent in the animals' physiological makeup, since there was a tendency for animals displaying a high rate of regeneration during the early stages to continue on a somewhat higher plane than the others during the postdepletion period.

Obviously these differences are not attributable to the size of the animals since all values were calculated on the basis of body weight. However, one contributing factor in cell volume regeneration during the post-depletion period was apparently the amount of the synthetic diet consumed, which observation was substantiated by the positive correlation coefficient obtained between food intake and cell volume regeneration. That it was not the only determinant was evident from the fact that the females actually consumed more food than the males on the basis of body weight although their cell volume regeneration, also on the basis of body weight, was distinctly lower.

The pre-bleeding mean normal cell volume was not a criterion of the animals' ability to replace lost blood cells. This conclusion was supported by the fact that those animals with a high normal cell volume per kilogram did not show a more rapid recovery than did the animals with a lower cell volume per kilogram. 
Based on data secured in this investigation, plus unpublished data covering other experiments performed in this laboratory on an additional group of dogs, the factors that were observed to influence cell volume regeneration were the degree of physiological stress induced by bleeding, the reserve of cell-building materials in the animal body, and food intake.

\section{Cell Volume Per Cent}

The data secured in the present investigation covering cell volume per cent confirm the observations of Adolph, Gerbasi, and Lepore (1933), who found that dilution of the plasma with fluid drawn from extravascular spaces occurred rapidly following blood loss. They reported the process was essentially complete within 22 minutes after hemorrhage. In the present short-term study, the dilution process had not been completed within 30 minutes of the start of bleeding, although appreciable dilution had occurred within that time, followed by complete adjustment within the succeeding 24 hours.

Robertson and Bock (1919) suggested, from their experience with wounded soldiers, that the rate of recovery following blood loss seemed to be dependent upon the quantity of reserve fluids in the tissues, since if patients were given large quantities of fluid by alimentary tract the blood volume increased quickly.

In the present long-term study it was observed that cell volume per cent was influenced by the number of cells produced and the size of the individual cells, from which it followed that the animals which regenerated the greatest number of cells, and at the same time the largest cells, showed the greatest degree of recovery in cell volume per cent. The males showed superiority over the females in this respect.

\section{Oxygen-combining Capacity}

The current investigation demonstrated the fact that the total blood volume does not return to normal after loss of significant amounts of blood. Therefore, reduction in the total oxygen-combining capacity of the blood may be more serious than the oxygen capacity, in terms of volumes per cent, would indicate.

In the absence of blood volume data, either cell count or oxygen capacity will serve, during the early stages of recovery, as an equally good measure of an animal's progress toward normality. After a period of time, during which the body builds cells that are smaller than normal, cell counts may be high and, taken by themselves, create an erroneous conception of the extent of improvement. Since total cell volume may show no increase, on the contrary even a decrease, the oxygen-combining capacity of the blood, per unit of volume, is the better measure of progress.

The data secured in the short-term study showed the decreases in cell volume per cent and in oxygen-combining capacity in volumes per 
cent to be of essentially the same magnitude, indicating that at least during the early stages of recovery the saturation of cells with hemoglobin was not altered following bleeding. The difficulties inherent in restoring hemoglobin to a normal level following loss of blood were evidenced by the fact that in the animals used in this study the level of oxygen capacity continued to decrease as a result of daily sampling.

In the long-term study also it was apparent that the weekly sampling, in which five per cent of the total blood volume was removed, largely offset any recovery that was made. The females actually lost ground in that their oxygen capacity was less at the end of the sixth post-bleeding week than it was at the end of the first week following hemorrhage. Although the males made somewhat better progress toward recovery than the females, their levels of oxygen capacity at the end of the sixth and at the end of the twelfth post-bleeding week were very little above the value observed at the end of the first post-bleeding week.

It was also. evident from the results of this research, plus unpublished data covering other experiments performed in this laboratory on an additional group of dogs, that the animals which had the highest pre-bleeding normal oxygen capacity were the furthest from their own normal levels at the end of the sixth post-bleeding week. The normal oxygen capacity of the blood is, therefore, plainly no indication of an animal's reserve of hemoglobin-building materials.

Recovery of the oxygen capacity of the blood is possibly related to the severity of the anemia produced. There is doubtless a critical level above which the oxygen capacity of the blood must be maintained even at the expense of other body tissues. Animals whose normal oxygen capacity is low will fall much more readily below this critical level; hence with such animals there is greater physiological need for recovery than with animals whose normal oxygen capacity is high. In other words, the loss of half the total blood volume in dogs with a high normal oxygen capacity will not serve as a stimulus to erythropoiesis in the same degree as in animals whose normal oxygen capacity is low.

Most workers in this field use the acid-hematin method rather than the more time-consuming oxygen-capacity method for observing the changing blood picture. Therefore, for the purpose of comparing the results secured in the present investigation with those reported by other workers, recovery in the oxygen-combining capacity of the blood was calculated in terms of grams of hemoglobin regenerated.

During the depletion period it was apparent that the males did possess some store of hemoglobin-building materials since they were capable of producing more than twice the amount of hemoglobin per kilogram per week during this period than they were during the remainder of the post-bleeding interval. The females, on the other hand, showed no such reserve, their rate of regeneration during the depletion period being actually slightly less than during the remainder of the postbleeding interval. 
The results cited above are contrary to the findings of Smith and Otis (1937) and Hubbell and Rose (1937), who in reporting studies on nutritional anemia in rats stated that female rats possessed greater reserves of hemoglobin-building materials than did the males.

It must be recognized that the earlier histories of the dogs used in this investigation were unknown; therefore, it is quite possible that the females had experienced one or more pregnancies which might have depleted or exhausted the reserves of hemoglobin-building materials.

The data obtained in the current research are in accord with results reported on observations made on male and female blood donors in that female donors are much less satisfactory than males. They develop anemia more quickly and also experience greater difficulty in recovery (Jones, Widing, and Nelson, 1931; Martin and Myers, 1934-35).

Some hemoglobin regeneration was observed on the synthetic diet, but no evidence was secured to indicate that the rate of regeneration was correlated with the level of food intake. Although the females actually consumed larger quantities of food per kilogram of body weight, their rate of hemoglobin regeneration was lower than that of the males.

Whipple and his co-workers conducted a long and comprehensive series of experiments on dogs to ascertain the effect of various foods on hemoglobin regeneration. Using a salmon-and-bread mixture as their basal diet, they observed a hemoglobin regeneration of 1.0 to 2.0 g. per two-week period. When this basal diet was supplemented with inorganic iron salts, Whipple and Robscheit-Robbins (1930) observed a maximum effect from the addition of $40.0 \mathrm{mg}$. of iron daily. When they used ferric citrate as the iron salt, they noted an increase in hemoglobin production amounting to $25.0 \mathrm{~g}$. per week.

In the present investigation, the mean daily iron intake, in the form of ferric citrate, was $21.6 \mathrm{mg}$. for the females and $24.6 \mathrm{mg}$. for the males. The mean hemoglobin production for the females per week was $4.7 \mathrm{~g}$., and for the males, 7.5 grams. Since the iron intake of the females was only 54 per cent, and of the males only 62 per cent of the amount of iron which Whipple and Robscheit-Robbins stated was the optimal level for hemoglobin production in dogs, it would be anticipated that the hemoglobin production for the animals used in the present studies would be proportionately less. On this basis the hemoglobin production expected of the females would have been $13.5 \mathrm{~g}$., and of the males, $15.4 \mathrm{~g}$. per week. Actually, the hemoglobin production of the females was only one third of this predicted amount, and of the males, only one half. These results would suggest that the limiting factor in the hemoglobin production in these animals was not the iron intake, but rather some factor other than iron needed for hemoglobin production which was supplied in more adequate amounts by the Whipple and Robscheit-Robbins' bread-and-salmon diet than by the synthetic diet used in the present investigation. 
McKay (1928) observed slow hemoglobin regeneration on a synthetic diet similar to that used in the present research, while Mayerson and Laurens (1930-31) found Cowgill's synthetic diet inadequate for maintaining a constant hemoglobin level in their anemic animals. None of these latter investigators supplemented their salt mixtures with copper as was done in the current investigation, which may have been an additional limiting factor in their experiments.

\section{Leucocytes}

In the present research there appeared to be a mild leucocytosis following hemorrhage similar to that reported by Keith (1919) and Jolly (1923). The latter suggested that the increase in leucocytes might be a temporary phenomenon, appearing shortly after hemorrhage, but not necessarily of uniform occurrence. The increase observed in this study, however, was not statistically significant when subjected to Student's " $t$ " test.

In the short-term study, possibly the determination made within 30 minutes of the start of bleeding did not allow sufficient time for increases to occur since the change which was actually observed at that time was a decrease in leucocytes. This was interpreted as resulting from the rapid dilution of the plasma with interstitial fluid. The slight increases in the mean values observed on the days following hemorrhage resulted in a large measure from increases noted for the one animal, number 47, on which four experiments were performed.

In the long-term study the females displayed more consistent evidence of a mild leucocytosis during the post-hemorrhagic period than did the males, but even among the females such evidence was not always present. Again, the observed increases were not found to be statistically significant.

Mayerson and Laurens (1930-31) reported that in only two of their experimental animals did they observe marked or consistent changes in either total white or differential counts. In them they noted a decrease in lymphocytes and a corresponding increase in neutrophiles.

In the third study, in which the animals were bled repeatedly, there was a very small increase in total neutrophiles, owing largely to a small but significant increase in the young band forms $(\mathrm{P}=<0.023)$. A corresponding decrease was observed in the mean values for total lymphocytes owing to decreases in both small and large forms.

\section{SUMMARY}

\section{Erythrocyte Counts}

In the short-term study, a 20 per cent decrease in erythrocyte counts was observed 24 hours after removing 26 per cent of the total blood volume. A continued decrease resulted thereafter from daily sampling. 
Data on cell counts produced no evidence of a reserve of preformed cells which could be drawn into the circulation in an emergency. In the longterm study, after removing approximately half of the total blood volume, the males' red cell counts had returned to normal in five weeks and the females', in seven weeks, after which counts for both males and females were distinctly above normal despite weekly sampling.

\section{Cell Size}

In the short-term study, mean corpuscular volume determinations, 30 minutes after the start of bleeding, produced a mean which was slightly above the pre-bleeding value, followed by decreases during the post-bleeding period to a level slightly below the pre-bleeding mean, five to seven days after hemorrhage. In the long-term study, cell diameter decreased throughout the first six weeks of the post-bleeding period, the females showing a more rapid decrease than the males. On a percentage basis, the decrease was 10 per cent for the females and 9 per cent for the males. Further small decreases were noted during the second six-week portion of the post-bleeding period. During the first six weeks of the post-bleeding period mean corpuscular volume decreased 17 per cent for the females, 15 per cent for the males. Further decreases during the balance of the post-bleeding period were observed for the females but not for the males. In a third study, on dogs bled four to five times and then observed for approximately four months, cell diameter decreased 24 per cent and mean corpuscular volume, 29 per cent.

\section{Total Cell Volume}

Based on total cell volume, also, no evidence was secured in the shortterm study to substantiate the existence of a reserve of preformed cells. Cell volume per kilogram decreased throughout the post-bleeding period as a result of daily sampling and reduction in cell size. In the long-term study, the females' cell volume per kilogram showed very little recovery cluring the first six weeks of the post-bleeding period following the initial decrease after the hemorrhages. For the males, recovery was somewhat more rapid. Neither males nor females made much progress during the remainder of the post-bleeding period.

The animals were fed a synthetic ration in amounts needed to maintain relatively constant body weight. Despite the fact that the mean food intake for the females was higher than for the males, the males showed more satisfactory progress during the recovery period than did the females.

The percentage of total blood volume removed from the males was slightly higher than that removed from the females, showing that the males were handled as drastically as the females in the matter of bleeding and sampling. 
The mean volume of cells regenerated during the first six weeks of the post-bleeding period was appreciably greater for the males than for the females, wide variations from animal to animal being evident in both groups. A significant positive correlation coefficient, +.6152 , between the percentage of cells removed and cell volume regenerated, indicates that physiological stress induced by bleeding influences cell volume regeneration.

Although the males appeared to possess greater stores of cell-building materials than did the females as shown by the fact that, based on body weight, they regenerated during the first two to three weeks following hemorrhage more than twice the volume of cells the females produced, the difference did not prove to be statistically significant. The exhaustion of these reserves was evidenced by the reduced capacity of both males and females to regenerate cell volume during the remainder of the postbleeding period, the males again showing slightly higher values than the females. Differences in the capacities of individual animals were apparent even after the exhaustion of cell reserves.

A significant positive correlation coefficient, +.7873 , between the volume of cells regenerated per kilogram of body weight and food intake per kilogram proved that during the later stages of recuperation -cell volume regeneration was in part controlled by food intake.

An insignificant correlation coefficient showed that no relationship existed between normal cell volume and the volume of cells regenerated in cubic centimeters per kilogram.

\section{Cell Volume Per Cent}

In the short-term study, the rapidity with which interstitial fluid was drawn into the blood stream following hemorrhage was demonstrated by an appreciable drop in cell volume per cent within 30 minutes after the start of hemorrhage. Complete adjustment followed within 24 hours. Further small decreases occurred throughout the remainder of the experiment owing to daily sampling and reduction in cell size. In the long-term study, the slow increase in cell volume per cent reflected the retarded recovery in total cell volume, the males making slightly better progress during the first six weeks of the post-bleeding period than the females. Neither males nor females showed much improvement during the second six-week portion of the post-bleeding period.

\section{Interrelationships between Red Cell Count, Size, and Volume of Cells}

In the long-term study, a significant correlation coefficient was obtained between cell count and cell diameter, -.7745, indicating a close relationship between cell diameter and the number of cells regenerated in hemorrhagic anemia, and thus verifying the earlier findings of Leichsenring and Hönig (1931). 
The significant correlation coefficient, +.4411 , between cell diameter and mean corpuscular volume showed no high degree of relationship between these two factors.

The correlation coefficient between cell count and cell volume in cubic centimeters per kilogram, +.3524 , while significant, indicated that cell count is not an entirely satisfactory measure of total cell volume.

\section{Oxygen-combining Capacity}

In the short-term study, the decrease observed 24 hours after hemorrhage was in close agreement with the drop in cell volume per cent. Although values noted on succeeding days were not in quite such close agreement, the data suggest that the concentration of hemoglobin in the cell is unaltered in the early stages of recuperation. In the long-term study, whereas the males at the end of the sixth post-bleeding week had made some progress toward recovery, the females actually showed a loss. Neither males nor females made any further progress during the second six-week interval. A significant correlation coefficient, +.5458 , between the normal oxygen capacity of the blood and the level at the end of the sixth post-bleeding week demonstrated the fact that the animals with the highest normal oxygen capacity were the furthest from their own normal levels at the end of this interval.

Recovery in oxygen capacity was calculated in terms of grams of hemoglobin regenerated. During the first two to three weeks of the post-bleeding period the males regenerated more than twice as much hemoglobin per kilogram per week as during the remainder of the postbleeding period. The females, on the other hand, did not demonstrate a reserve of hemoglobin-building materials. The males showed superiority over the females during the entire post-bleeding period. Hemoglobin regeneration was not related to the level of food intake as shown by the insignificant correlation coefficient obtained between these two factors.

\section{Leucocytes}

In the short-term study, the apparent leucocytosis noted in the postbleeding period was shown to be statistically insignificant. In the longterm study, both females and males showed some evidence of leucocytosis, maximum counts being observed at the end of the first and second post-bleeding weeks, respectively, with decreases toward normal levels throughout the remainder of the post-bleeding period. Again the observed increase was not significant.

In the third study, the animals showed no increase in the total leucocytes after repeated hemorrhage. The differential leucocyte count showed a small but significant increase in the percentage of the young band-form neutrophiles $(\mathrm{P}=<0.023)$, accompanied by a decrease in the total lymphocytes involving both small and large forms. 


\section{BLOOD PLASMA}

\section{PLASMA VOLUME}

I $^{\mathrm{N}}$ THE Discussion of cell volume per cent, from data secured in the short-term study, it was pointed out that there was a rapid withdrawal of interstitial fluid from the tissues to restore plasma volume to normal. Within 30 minutes of the start of hemorrhage, in which 20 per cent of the total blood volume had been removed, the mean plasma volume was observed to be 773.5 cc., compared with a pre-bleeding mean of $889.0 \mathrm{cc}$., a decrease of only 13 per cent. When these volumes were calculated on the basis of the mean body weight of the animals, the level 30 minutes after the start of bleeding was $39.7 \mathrm{cc}$. per kilogram, as against a mean pre-bleeding value of $45.0 \mathrm{cc}$. per kilogram (table 35). Equilibrium had been established 24 hours later at a level slightly above the pre-bleeding level (46.0 cc. per kilogram), and thereafter increases in plasma volume per kilogram tended to balance the losses in cell volume owing to daily sampling. However, at no time did the increase in plasma volume compensate completely for the loss in cells, with the result that the total blood volume remained distinctly below normal throughout the postbleeding period (table 50).

Table 35. Values for Plasma Volume, in Cubic Centimeters per Kilogram (Short-term study)

\begin{tabular}{|c|c|c|c|c|c|c|}
\hline \multirow{2}{*}{$\begin{array}{l}\text { Dog } \\
\text { No. }\end{array}$} & \multirow{2}{*}{$\begin{array}{l}\text { Last pre- } \\
\text { bleeding }\end{array}$} & \multirow{2}{*}{$\begin{array}{l}\text { Within } \\
30 \text { minutes } \\
\text { of start of } \\
\text { bleeding }\end{array}$} & \multicolumn{4}{|c|}{ Post-bleeding period } \\
\hline & & & $I \mathrm{~d} \alpha \mathrm{y}$ & 2 days & 3 days & 5.7 days \\
\hline \multicolumn{7}{|l|}{ Males } \\
\hline \multirow[t]{2}{*}{44} & 46.6 & 41.9 & 46.7 & 47.4 & 46.4 & 46.0 \\
\hline & 48.3 & 37.3 & 45.4 & 45.4 & 46.6 & 48.3 \\
\hline \multirow[t]{2}{*}{45} & 39.8 & $\ldots \ldots \ldots \ldots$ & 44.0 & 44.5 & 45.8 & 44.6 \\
\hline & 41.5 & 33.0 & 43.3 & 42.2 & $42.9^{\circ}$ & 44.5 \\
\hline \multirow[t]{2}{*}{46} & 47.1 & 42.2 & 52.4 & 50.1 & 54.2 & 49.2 \\
\hline & 48.6 & 39.4 & 45.3 & 48.9 & 50.8 & 51.3 \\
\hline \multirow[t]{4}{*}{47} & 39.1 & 40.5 & 42.4 & 37.6 & 37.5 & 44.6 \\
\hline & 45.9 & 43.3 & 45.4 & 48.5 & 49.7 & 49.8 \\
\hline & 47.1 & 39.2 & 43.4 & 46.0 & 43.4 & 44.9 \\
\hline & 46.0 & 40.6 & 51.2 & 47.0 & 50.5 & 50.2 \\
\hline Mean & 45.0 & 39.7 & 46.0 & 45.8 & 46.8 & 47.3 \\
\hline
\end{tabular}

For the female dogs used in the long-term study, the mean normal plasma volume was $35.3 \mathrm{cc}$. per kilogram (table 36). One week after the removal of approximately one half the established total blood volume, plasma volume had increased to $38.3 \mathrm{cc}$., with thereafter a gradual de- 
crease until at the end of six weeks the mean plasma volume was found to be $36.6 \mathrm{cc}$. per kilogram. For the three females observed for 12 weeks, the mean at the end of the twelfth week was 36.7 cc., contrasted with a mean of $37.8 \mathrm{cc}$. at the end of the sixth week.

Table 36. Values for Plasma Volume, in Cubic Centimeters per Kilogram (Long-term study)

\begin{tabular}{|c|c|c|c|c|c|c|c|c|c|}
\hline \multirow{2}{*}{$\begin{array}{c}\text { Dog } \\
\text { No. }\end{array}$} & \multirow{2}{*}{$\begin{array}{c}\text { Pre- } \\
\text { bleeding } \\
\text { normal }\end{array}$} & \multicolumn{8}{|c|}{ Post-bleeding period } \\
\hline & & $1-5$ days & 1 wk. & 2 wks. & 3 wks. & 4 wks. & 5 wks. & 6 wks. & $12 \mathrm{wks}$. \\
\hline Females & & & & & & & & & - \\
\hline 20 & 41.4 & 40.6 & 44.5 & 41.4 & 44.2 & 39.7 & 42.0 & 45.1 & 44.4 \\
\hline 21 & 37.7 & 47.3 & 38.6 & 42.3 & 32.2 & 35.4 & 37.0 & 37.9 & $\ldots$ \\
\hline 22 & 33.8 & 38.7 & 33.1 & 36.0 & 36.8 & 34.3 & 34.1 & 32.0 & $\ldots \ldots . . . .$. \\
\hline 19 & 28.9 & 41.7 & 34.0 & 34.2 & $\ldots$ & 37.8 & 33.0 & 32.2 & 30.6 \\
\hline 32 & 34.8 & 37.5 & 41.4 & 38.1 & 38.1 & 37.6 & 37.2 & 36.0 & 35.0 \\
\hline Mean & 35.3 & 41.2 & 38.3 & 38.4 & 37.8 & 37.0 & 36.7 & 36.6 & 36.7 \\
\hline \multicolumn{10}{|l|}{ Males } \\
\hline 17 & 37.2 & 34.8 & 41.3 & 41.7 & 40.0 & 37.8 & 35.7 & 40.9 & 46.0 \\
\hline 18 & 31.5 & 38.1 & 33.3 & 30.6 & 37.6 & 38.2 & 34.8 & 35.1 & 36.0 \\
\hline 30 & 45.9 & 49.6 & 49.0 & 48.7 & 47.2 & 43.2 & 46.7 & 46.4 & 40.2 \\
\hline 31 & 41.0 & 41.6 & 44.7 & 43.0 & 41.7 & 40.2 & 40.4 & 40.4 & 38.1 \\
\hline Mean & 38.9 & 41.0 & 42.1 & 41.0 & 41.6 & 39.8 & 39.4 & 40.7 & 40.0 \\
\hline
\end{tabular}

The mean normal plasma volume for the males in this group was 38.9 cc. per kilogram, a somewhat higher value than that observed for the females. The plasma volume had reached a mean level of $42.1 \mathrm{cc}$. by the end of the first post-bleeding week, $40.7 \mathrm{cc}$. by the end of the sixth week, with a further slight decrease to $40.0 \mathrm{cc}$. by the end of the twelfth week. Evidently the animal body is capable of adjusting to an appreciably smaller total blood volume following hemorrhage.

\section{BLOOD PLASMA CONSTITUENTS}

Protein Nitrogcn.--In the short-term study, the plasma protein nitrogen, measured in grams per 100 cubic centimeters, did not return to normal during the post-bleeding period (table 37). The mean prebleeding value was $1.07 \mathrm{~g}$. per $100 \mathrm{cc}$. of plasma. Within 30 minutes of the start of hemorrhage the mean was $1.00 \mathrm{~g}$., and throughout the remainder of the post-bleeding period the values observed were slightly below this level.

Multiplying the grams of protein nitrogen per cubic centimeter by the total plasma volume in cubic centimeters, to secure the total plasma protein nitrogen in the total circulation, a value was obtained which showed a return to normal 24 hours after hemorrhage (table 38 ). The pre-bleeding mean for the total protein nitrogen was $9.02 \mathrm{~g}$., compared with the first post-bleeding value, secured 24 hours after hemorrhage, 
Table 37. Values for Protein Nitrogen in Plasma, in Grams per 100 Cubic Centimeters (Short-term study)

\begin{tabular}{|c|c|c|c|c|c|c|}
\hline \multirow{2}{*}{$\begin{array}{l}\text { Dog } \\
\text { No. }\end{array}$} & \multirow{2}{*}{$\begin{array}{l}\text { Last pre- } \\
\text { bleeding }\end{array}$} & \multirow{2}{*}{$\begin{array}{l}\text { Within } \\
30 \text { minutes } \\
\text { of start of } \\
\text { bleeding }\end{array}$} & \multicolumn{4}{|c|}{ Post-bleeding period } \\
\hline & & & 1 day & 2 days & 3 days & 5-7 days \\
\hline \multicolumn{7}{|l|}{ Males } \\
\hline \multirow[t]{2}{*}{44} & 1.040 & 0.986 & 1.049 & 1.001 & 0.978 & 1.003 \\
\hline & 1.085 & 1.060 & 1.025 & 1.026 & 0.979 & 0.981 \\
\hline \multirow[t]{2}{*}{45} & 1.089 & $\ldots \ldots \ldots \ldots \ldots \ldots$ & 1.050 & 0.922 & 0.865 & 0.959 \\
\hline & 1.042 & 1.034 & ......................... & 0.945 & 0.959 & 0.959 \\
\hline \multirow[t]{2}{*}{46} & 1.140 & 1.104 & 1.064 & 1.072 & 1.038 & 1.007 \\
\hline & 1.047 & 1.005 & 1.005 & 0.982 & 1.032 & 0.971 \\
\hline \multirow[t]{4}{*}{47} & 1.062 & 0.819 & 0.941 & 0.944 & 0.924 & 0.874 \\
\hline & 1.066 & 1.019 & 0.953 & 0.971 & 1.042 & 1.024 \\
\hline & 1.072 & 0.974 & 0.922 & 1.026 & 1.015 & 1.018 \\
\hline & 1.046 & $\ldots$ & 0.978 & 1.015 & 1.001 & 1.000 \\
\hline Mean & 1.069 & 1.000 & 0.996 & 0.990 & 0.983 & 0.980 \\
\hline
\end{tabular}

Table 38. Comparison of the Mean Total Amounts of Each of the Plasma Constituents in the Circulation Before and After Hemorrhage*

(Short-term study)

\begin{tabular}{|c|c|c|c|c|c|c|c|c|c|}
\hline & \multirow[b]{2}{*}{ - } & \multicolumn{2}{|c|}{ Nitrogen } & \multicolumn{3}{|c|}{ Calcium } & \multicolumn{3}{|c|}{ Phosphorus } \\
\hline & & $\begin{array}{l}\text { Pro- } \\
\text { tein } \\
\text { in } \\
\text { grams }\end{array}$ & $\begin{array}{l}\text { Non- } \\
\text { pro- } \\
\text { tein in } \\
\text { grams }\end{array}$ & $\begin{array}{c}\text { Dif- } \\
\text { fus- } \\
\text { ible } \\
\text { in } \mathrm{mg} .\end{array}$ & $\begin{array}{l}\text { Non- } \\
\text { diffus- } \\
\text { ible } \\
\text { in } \mathrm{mg} .\end{array}$ & $\begin{array}{l}\text { Total } \\
\text { in } \mathrm{mg} .\end{array}$ & $\begin{array}{l}\text { Lipide } \\
\text { in } \mathrm{mg} \text {. }\end{array}$ & $\begin{array}{l}\text { Inor- } \\
\text { ganic } \\
\text { in } \mathrm{mg} .\end{array}$ & $\begin{array}{l}\text { Total } \\
\text { in } \mathrm{mg} .\end{array}$ \\
\hline \multirow{4}{*}{$\begin{array}{l}\text { Pre-bleeding values } \\
\text {. }\end{array}$} & 1 & 8.60 & 0.228 & 62.8 & 44.9 & 107.7 & 135.4 & 41.4 & 184.2 \\
\hline & 2 & 8.86 & 0.211 & 65.4 & 37.6 & 96.0 & 137.0 & 44.8 & 194.2 \\
\hline & 3 & 9.31 & 0.229 & 66.0 & 35.6 & 101.6 & 150.8 & 47.4 & 212.2 \\
\hline & 4 & 9.31 & 0.225 & 70.4 & 42.1 & 112.5 & 155.3 & 46.2 & 209.7 \\
\hline \multicolumn{2}{|l|}{ Mean } & 9.02 & 0.223 & 66.2 & 40.4 & 104.4 & 144.6 & 45.0 & 200.1 \\
\hline Within 30 minutes of & & 7.96 & 0.197 & 57.6 & 36.1 & 93.7 & 122.0 & 38.7 & 182.7 \\
\hline \multirow[t]{5}{*}{ Post-bleeding values } & 1 & 9.06 & 0.221 & 67.9 & 47.4 & 115.3 & 155.4 & 45.6 & 196.4 \\
\hline & 2 & 9.03 & 0.209 & 67.8 & 45.4 & 113.2 & 155.6 & 46.9 & 211.0 \\
\hline & 3 & 9.20 & 0.226 & 67.0 & 40.4 & 107.4 & 152.7 & 48.4 & 209.2 \\
\hline & 4 & 9.18 & 0.224 & 71.5 & 40.9 & 112.4 & 152.0 & 46.3 & 210.1 \\
\hline & 5 & 9.52 & 0.229 & 71.0 & 46.5 & 117.5 & 152.4 & 50.2 & 217.2 \\
\hline Mean & - & 9.20 & 0.222 & 69.0 & 44.1 & 113.2 & 153.6 & 47.5 & 208.8 \\
\hline
\end{tabular}

* Each value represents the mean result of the 10 experiments, and was obtained by multiplying the amount per cubic centimeter by the plasma volume in cubic centimeters. The values obtained on samples secured within 30 minutes of the institution of bleeding were not included in the post-bleeding mean. 
of $9.06 \mathrm{~g}$., and a mean of $9.20 \mathrm{~g}$. for the post-bleeding period. Considered solely on the basis of the amount of protein nitrogen per unit of volume, this recovery is not apparent because of the increase in plasma volume.

Nonprotein Nitrogen.-As with the protein nitrogen, the nonprotein nitrogen level was slightly below the pre-bleeding normal during the post-bleeding period (table 39). The mean pre-bleeding value obtained was $27.0 \mathrm{mg}$. per $100 \mathrm{cc}$. plasma. A similar mean value was observed again within 30 minutes of the start of hemorrhage, $26.7 \mathrm{mg}$.,

Table 39. Values for Nonprotein Nitrogen in Plasma, in Milligrams per 100 Cubic Centimeters

(Short-term study)

\begin{tabular}{|c|c|c|c|c|c|c|}
\hline \multirow{2}{*}{$\begin{array}{l}\text { Dog } \\
\text { No. }\end{array}$} & \multirow{2}{*}{$\begin{array}{l}\text { Last pre- } \\
\text { bleeding }\end{array}$} & \multirow{2}{*}{$\begin{array}{l}\text { Within } \\
30 \text { minutes } \\
\text { of start of } \\
\text { bleeding }\end{array}$} & \multicolumn{4}{|c|}{ Post-bleeding period } \\
\hline & & & 1 day & 2 days & 3 days & 5-7 days \\
\hline \multicolumn{7}{|l|}{ Males } \\
\hline \multirow[t]{2}{*}{44} & 20.7 & 21.6 & 20.4 & 18.9 & 18.7 & 18.6 \\
\hline & 19.3 & 20.3 & 18.1 & 18.0 & 17.2 & 18.3 \\
\hline \multirow[t]{2}{*}{45} & 31.8 & 32.1 & 31.4 & 26.0 & 28.6 & 30.9 \\
\hline & 22.5 & 20.5 & 24.2 & 22.6 & 22.3 & 22.8 \\
\hline \multirow[t]{2}{*}{46} & 32.8 & 28.4 & 23.7 & 24.0 & 26.7 & 25.0 \\
\hline & 25.5 & 26.6 & 22.0 & 25.1 & 27.7 & 24.1 \\
\hline \multirow[t]{4}{*}{47} & 33.9 & 31.6 & 33.3 & 29.0 & 26.6 & 24.4 \\
\hline & 34.6 & 32.9 & 29.1 & 27.5 & 27.9 & 29.5 \\
\hline & 26.8 & 28.6 & 27.3 & 24.4 & 27.4 & 27.9 \\
\hline & 22.1 & 24.5 & 24.5 & 20.8 & 22.8 & 20.7 \\
\hline Mean & 27.0 & 26.7 & 25.4 & 23.6 & 24.6 & 24.2 \\
\hline
\end{tabular}

with a decrease to $25.4 \mathrm{mg}$. 24 hours later, to a still lower value, 23.6 mg., followed by slight variations throughout the balance of the postbleeding period. When, as in the case of the protein nitrogen, the total amount of nonprotein nitrogen in the circulation was calculated, a surprising agreement was noted between pre-bleeding and post-bleeding means, the value for the former being $0.223 \mathrm{~g}$., for the latter, $0.222 \mathrm{~g}$., in the total plasma (table 38).

In the long-term study, determinations on total nitrogen and nonprotein nitrogen were made on whole blood. The resulting values will, therefore, be discussed in the section to follow.

Phosphorus and Calcium Fractions.-Although lipide phosphorus per unit volume of plasma decreased within 30 minutes of the start of bleeding, and then returned almost to normal within 24 hours, this level was not maintained throughout the post-bleeding period. The mean pre- 
Table 40. Values for Lipide Phosphorus in Plasma, in Milligrams per 100 Cubic Centimeters

(Short-term study)

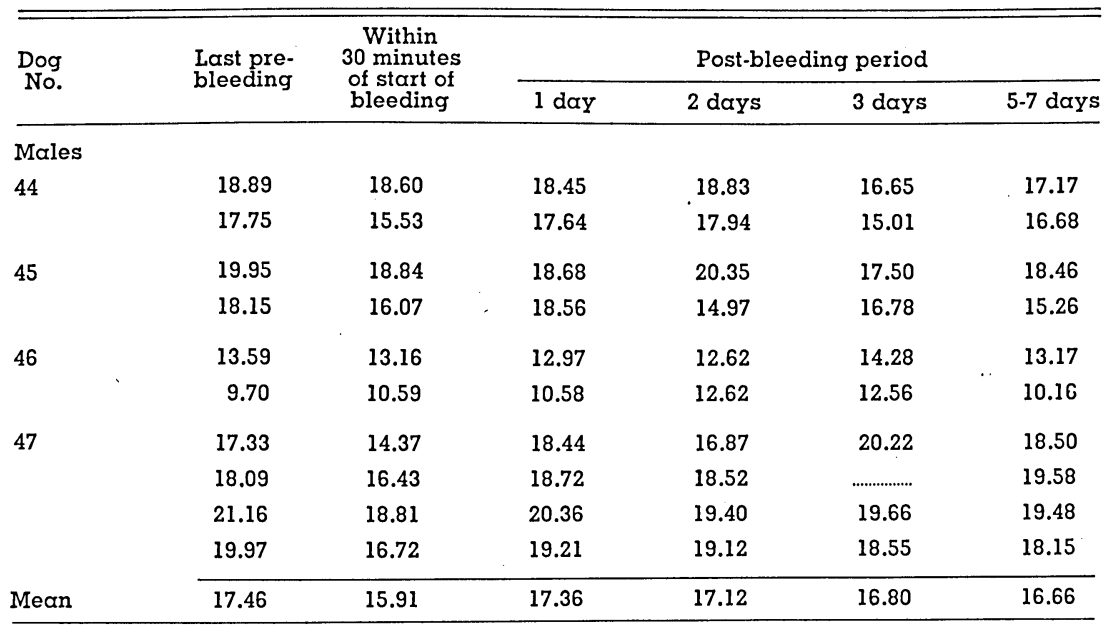

bleeding level was $17.5 \mathrm{mg}$. per $100 \mathrm{cc}$., which decreased to $15.9 \mathrm{mg}$., and recovered to $17.4 \mathrm{mg}$. 24 hours after hemorrhage (table 40). On the second and third days, the values noted were 17.1 and $16.8 \mathrm{mg}$., respectively.

The level for inorganic phosphorus showed, within 30 minutes of the start of bleeding, the effect of dilution similar to that observed in the other factors studied (table 41). During the balance of the week, the

Table 41. Values for Inorganic Phosphorus in Plasma, in Milligrams per 100 Cubic Centimeters

(Short-term study)

\begin{tabular}{|c|c|c|c|c|c|c|}
\hline \multirow{2}{*}{$\begin{array}{l}\text { Dog } \\
\text { No. }\end{array}$} & \multirow{2}{*}{$\begin{array}{l}\text { Last pre- } \\
\text { bleeding }\end{array}$} & \multirow{2}{*}{$\begin{array}{l}\text { Within } \\
30 \text { minutes } \\
\text { of start of } \\
\text { bleeding }\end{array}$} & \multicolumn{4}{|c|}{ Post-bleeding period } \\
\hline & & & $1 \mathrm{day}$ & 2 days & 3 days & $5-7$ days \\
\hline \multicolumn{7}{|l|}{ Males } \\
\hline \multirow[t]{2}{*}{44} & 6.49 & 6.01 & 5.79 & 6.53 & 5.67 & 5.96 \\
\hline & 5.76 & 5.36 & 6.13 & 5.93 & 5.43 & 5.62 \\
\hline \multirow[t]{2}{*}{45} & 6.93 & 5.13 & 5.55 & 5.22 & 5.49 & 5.17 \\
\hline & 5.84 & 5.05 & 5.27 & 4.65 & 5.46 & 4.95 \\
\hline \multirow[t]{2}{*}{46} & 5.93 & 4.71 & 4.46 & 4.99 & 5.08 & 4.96 \\
\hline & 4.51 & 5.10 & 4.49 & 4.62 & 4.86 & 4.21 \\
\hline \multirow[t]{4}{*}{47} & 4.04 & 3.72 & 4.25 & 4.15 & 3.64 & 4.34 \\
\hline & 4.72 & 4.49 & $4.10^{\circ}$ & 4.80 & $\ldots+. . . . . .$. & 5.54 \\
\hline & 5.06 & 4.20 & 4.98 & 4.96 & 4.88 & 5.20 \\
\hline & 5.79 & 5.00 & 4.77 & 4.82 & 4.90 & 4.12 \\
\hline Mean & 5.51 & 4.88 & 4.98 & 5.07 & 5.04 & 5.01 \\
\hline
\end{tabular}


values were slightly below the value obtained for the pre-bleeding sample. The mean pre-bleeding value for the 10 experiments was $5.51 \mathrm{mg}$. per $100 \mathrm{cc}$., which decreased to $4.88 \mathrm{mg}$. within 30 minutes of the start of bleeding, and remained approximately unchanged for the balance of the post-bleeding period.

Total phosphorus data for these animals are given in table 42. It will be noted that the means are in all cases slightly higher than the

Table 42. Values for Total Phosphorus in Plasma, in Milligrams per 100 Cubic Centimeters

(Short-term study)

\begin{tabular}{|c|c|c|c|c|c|c|}
\hline \multirow{2}{*}{$\begin{array}{l}\text { Dog } \\
\text { No. }\end{array}$} & \multirow{2}{*}{$\begin{array}{l}\text { Last pre- } \\
\text { bleeding }\end{array}$} & \multirow{2}{*}{$\begin{array}{l}\text { Within } \\
30 \text { minutes } \\
\text { of start of } \\
\text { bleeding }\end{array}$} & \multicolumn{4}{|c|}{ Post-bleeding period } \\
\hline & & & 1 day & 2 days & 3 days & 5-7 days \\
\hline \multicolumn{7}{|l|}{ Males } \\
\hline \multirow[t]{2}{*}{44} & 26.85 & 24.74 & 23.89 & 25.00 & 23.78 & 23.84 \\
\hline & 23.54 & 22.49 & 24.91 & 23.77 & 22.49 & 22.36 \\
\hline \multirow[t]{2}{*}{45} & 28.08 & 24.31 & 24.86 & 27.38 & 24.66 & 24.62 \\
\hline & 23.37 & 21.05 & 23.61 & 18.99 & 20.59 & 21.24 \\
\hline \multirow[t]{2}{*}{46} & 20.76 & 19.00 & 20.34 & 20.17 & 20.35 & 19.86 \\
\hline & 17.00 & 16.69 & 18.14 & 17.45 & 19.38 & 16.59 \\
\hline \multirow[t]{4}{*}{47} & 20.46 & 17.67 & 21.35 & 20.62 & 26.40 & 22.02 \\
\hline & 24.42 & 21.49 & 22.76 & 25.14 & 23.70 & 26.12 \\
\hline & 26.23 & 23.50 & 26.12 & 25.66 & 24.80 & 27.34 \\
\hline & 26.36 & 22.89 & 24.60 & 23.40 & 23.45 & 23.14 \\
\hline Mean & 23.71 & 21.38 & 23.06 & 22.76 & 22.96 & 22.71 \\
\hline
\end{tabular}

sum of the means for the lipide and inorganic phosphorus fractions, indicating the presence in the blood, in relatively constant amounts, of other phosphorus-containing compounds.

The means for diffusible and nondiffusible calcium, observed within 30 minutes of the start of bleeding, also show the effects of dilution. For diffusible calcium, the mean pre-bleeding value was $7.71 \mathrm{mg}$. (table 43), which decreased within this 30-minute interval to 7.33 milligrams. During the rest of the week values noted were slightly below the pre-bleeding level, namely, 7.44, 7.40, 7.10, and $7.60 \mathrm{mg}$., respectively.

The mean pre-bleeding value for nondiffusible calcium was $5.00 \mathrm{mg}$. per $100 \mathrm{cc}$. of plasma (table 44), which decreased within 30 minutes after the start of bleeding to 4.88 milligrams; 24 hours thereafter a mean of $5.42 \mathrm{mg}$. was obtained, followed on the second day by a mean value of 4.99 milligrams. Although the extent to which these values were influenced by the loss of one sample on each of the first three post-bleeding days cannot be determined, there appears to be a clear tendency for the level of the nondiffusible calcium to decrease after the first post-bleeding day to a value below the pre-bleeding mean. 
Table 43. Values for Diffusible Calcium in Plasma, in Milligrams per 100 Cubic Centimeters

(Short-term study)

\begin{tabular}{|c|c|c|c|c|c|c|}
\hline \multirow{2}{*}{$\begin{array}{l}\text { Dog } \\
\text { No. }\end{array}$} & \multirow{2}{*}{$\begin{array}{l}\text { Last pre- } \\
\text { bleeding }\end{array}$} & \multirow{2}{*}{$\begin{array}{l}\text { Within } \\
30 \text { minutes } \\
\text { of start of } \\
\text { bleeding }\end{array}$} & \multicolumn{4}{|c|}{ Post-bleeding period } \\
\hline & & & $1 \mathrm{day}$ & 2 days & 3 days & 5-7 days \\
\hline \multicolumn{7}{|l|}{ Males } \\
\hline \multirow[t]{2}{*}{44} & 8.82 & 8.16 & 6.82 & 7.66 & 8.26 & 8.57 \\
\hline & 6.29 & 6.03 & 6.27 & 6.11 & 5.98 & 6.29 \\
\hline \multirow[t]{2}{*}{45} & 8.70 & 7.57 & 8.54 & 7.66 & 7.68 & 7.53 \\
\hline & 7.69 & 7.50 & 9.29 & 8.14 & 8.31 & 8.37 \\
\hline \multirow[t]{2}{*}{46} & 7.87 & 7.82 & 7.80 & 7.93 & 8.19 & 8.45 \\
\hline & 8.45 & 8.58 & 6.66 & 6.82 & 6.25 & 6.69 \\
\hline \multirow[t]{4}{*}{47} & 5.92 & 6.31 & 6.53 & 6.78 & 5.87 & 6.55 \\
\hline & 9.47 & 7.72 & 7.64 & 8.04 & 7.30 & 8.29 \\
\hline & 7.80 & 7.83 & 7.98 & 7.96 & 7.39 & 7.76 \\
\hline & 6.08 & 5.78 & 6.87 & 6.95 & 5.74 & 7.49 \\
\hline Mean & 7.71 & 7.33 & 7.44 & 7.40 & 7.10 & 7.60 \\
\hline
\end{tabular}

Again, as in the case of the protein nitrogen and nonprotein nitrogen, if the total amount of these various fractions in the circulating plasma were calculated by multiplying the amount of each, per unit of volume, by the total volume of the plasma, a complete recovery of the total amount in the circulation would be observed (table 38). In fact, for all fractions, slightly higher means were apparent in the post-bleeding period when compared with the pre-bleeding values. The pre-bleeding and post-bleeding totals for the various factors in the circulating plasma

Table 44. Values for Nondiffusible Calcium in Plasma, in Milligrams per 100 Cubic Centimeters

(Short-term study)

\begin{tabular}{|c|c|c|c|c|c|c|}
\hline \multirow{2}{*}{$\begin{array}{l}\text { Dog } \\
\text { No. }\end{array}$} & \multirow{2}{*}{$\begin{array}{l}\text { Last pre- } \\
\text { bleeding }\end{array}$} & \multirow{2}{*}{$\begin{array}{l}\text { Within } \\
30 \text { minutes } \\
\text { of start of } \\
\text { bleeding }\end{array}$} & \multicolumn{4}{|c|}{ Post-bleeding period } \\
\hline & & & 1 day & 2 days & 3 days & 5-7 days \\
\hline \multicolumn{7}{|l|}{ Males } \\
\hline \multirow[t]{2}{*}{44} & 5.44 & 5.93 & 6.83 & $\ldots \ldots \ldots \ldots$ & 3.86 & 4.73 \\
\hline & 4.80 & 5.42 & 4.90 & 6.37 & 5.47 & 3.80 \\
\hline \multirow[t]{2}{*}{45} & 6.71 & 6.49 & 4.05 & 6.59 & 4.95 & 4.77 \\
\hline & 4.97 & 3.56 & .............. & 3.19 & 3.21 & 4.58 \\
\hline \multirow{2}{*}{46} & 4.37 & 3.49 & 4.68 & 4.58 & 4.28 & 4.21 \\
\hline & 3.54 & 4.47 & 4.09 & 3.92 & 3.81 & 4.48 \\
\hline \multirow[t]{4}{*}{47} & 5.39 & 5.00 & 6.66 & 4.95 & 4.02 & 4.26 \\
\hline & 4.76 & 5.81 & 6.74 & 5.16 & $\ldots$ & 4.53 \\
\hline & 4.49 & 4.01 & 5.76 & 4.33 & 5.20 & 4.44 \\
\hline & 5.55 & 4.57 & 5.06 & 5.84 & 4.11 & 4.62 \\
\hline Mean & 5.00 & 4.88 & 5.42 & 4.99 & 4.32 & 4.44 \\
\hline
\end{tabular}


follow: lipide phosphorus, 144.6 and 153.6 milligrams; inorganic phosphorus, 45.0 and 47.5 milligrams; total phosphorus, 200.1 and 208.8 milligrams; diffusible calcium, 66.2 and 69.0 milligrams; nondiffusible calcium, 40.4 and 44.1 milligrams; and total calcium, 104.4 and 113.2 milligrams, respectively.

Significant positive correlations were obtained between the levels of plasma protein and inorganic phosphorus before hemorrhage, +.3248 , and after hemorrhage, +.3487 .

After hemorrhage, significant positive correlations were observed between lipide phosphorus and nonprotein nitrogen, +.2575 ; between lipide phosphorus and inorganic phosphorus, +.4371 ; between lipide phosphorus and diffusible calcium, +.3077 ; and between inorganic phosphorus and diffusible calcium, +.3897 , although no such relationships were apparent in the pre-bleeding levels.

A significant negative correlation was obtained between pre-bleeding values for diffusible and nondiffusible calcium, -.3787 , but not between the post-bleeding values.

Plasma Protcin Fractions: Albumin.-In the fourth study, working with three male dogs, the effect of repeated bleeding on the level of the plasma proteins was investigated. It was observed that the level of albumin in the plasma was restored rapidly. The mean normal level was $3.98 \mathrm{~g}$. per $100 \mathrm{cc}$. of plasma (table 45), but 48 hours after the loss of one fourth to one half of the total blood volume (in one or two bleedings) the mean level was found to be 4.05 grams. Although each animal was bled two or three times more, removing at each bleeding one fourth of the total blood volume, at no time was the mean albumin level found to be below normal. As a matter of fact, a mean distinctly higher than normal, $4.70 \mathrm{~g}$., was obtained following the third hemorrhage.

Globulin.-The globulin level, on the other hand, was apparently restored with difficulty (table 46). The mean normal value was found to be $2.46 \mathrm{~g}$. per $100 \mathrm{cc}$. of plasma. Forty-eight hours after the first hemorrhage the mean value was $1.89 \mathrm{~g}$., and after succeeding hemorrhages, mean values of 2.17 and $2.00 \mathrm{~g}$. were noted. Two of the animals were subjected to a fourth bleeding. In these dogs, whose normal levels produced a mean of $2.14 \mathrm{~g}$., the globulin level increased after each hemorrhage as shown by the means-1.84, 2.04, 2.24, and 2.50 grams. The results cited indicate that the animal organism evidently can adapt itself to losses of globulin by increasingly rapid regeneration and finally attain a value actually higher than normal.

Albumin-Globulin Ratio.-Alterations in the albumin-globulin ratio, corresponding with these changing levels, ranged from a mean prebleeding normal of 1.69 to a mean of 2.53 following the third hemorrhage (table 47). 
Table 45. Albumin in Plasma, in Grams per 100 Cubic Centimeters

\begin{tabular}{|c|c|c|c|c|c|c|c|c|c|}
\hline \multirow{2}{*}{$\begin{array}{l}\text { Dog } \\
\text { No. }\end{array}$} & \multirow{2}{*}{$\begin{array}{l}\text { Pre- } \\
\text { bleed- } \\
\text { ing } \\
\text { normal }\end{array}$} & \multicolumn{2}{|c|}{$\begin{array}{c}\text { After first } \\
\text { hemorrhage }\end{array}$} & \multicolumn{2}{|c|}{$\begin{array}{l}\text { After second } \\
\text { hemorrhage }\end{array}$} & \multicolumn{2}{|c|}{$\begin{array}{c}\text { After third } \\
\text { hemorrhage }\end{array}$} & \multicolumn{2}{|c|}{$\begin{array}{l}\text { After fourth } \\
\text { hemorrhage }\end{array}$} \\
\hline & & No. days & Āmount & No. days & Āmount & No. days & Amount & No. days & Amount \\
\hline \multicolumn{10}{|l|}{ Males } \\
\hline \multirow[t]{4}{*}{55} & 3.78 & 2 & 3.97 & 2 & 2.98 & 2 & 4.66 & 3 & 4.08 \\
\hline & 3.59 & $\ldots . .$. & $\ldots \ldots \ldots$ & 7 & 3.70 & 10 & 4.42 & 10 & 4.32 \\
\hline & .............. & $\ldots . .$. & $\ldots \ldots \ldots \ldots$ & 14 & 4.19 & 17 & 4.12 & $\ldots . .$. & $\ldots \ldots . . . . . .$. \\
\hline & $\ldots \cdots \cdots \cdots \cdot$ & $\ldots . .$. & $\ldots+\cdots \cdot \cdots \cdot \cdots$ & $\cdots \cdots$ & $\ldots \ldots \ldots$ & 24 & 4.47 & $\ldots . \cdots$ & 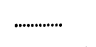 \\
\hline Mean & 3.69 & $\ldots . .$. & 3.97 & $\ldots . .$. & 3.62 & $\ldots \ldots \cdot$ & 4.42 & $\ldots . .$. & 4.20 \\
\hline \multirow[t]{3}{*}{54} & 4.01 & 2 & 3.89 & 2 & 3.60 & 3 & 4.62 & 2 & 4.05 \\
\hline & ................... & $\ldots . .$. & $\ldots \ldots \ldots \ldots \ldots$ & 9 & 4.14 & 10 & 4.41 & 8 & 4.42 \\
\hline & $\ldots \ldots \ldots \ldots$ & $\ldots \cdots \cdot$ & $\ldots+\cdots \cdots \cdot \cdot \cdot$ & 16 & 4.23 & $\ldots . .$. & $\ldots . \cdots \cdots \cdots$ & $\ldots . .$. & $\ldots+\cdots \cdot \cdots \cdot \cdots$ \\
\hline Mean & 4.01 & $\cdots . .$. & 3.89 & $\ldots . .$. & 3.99 & $\ldots \ldots$ & 4.52 & $\cdots \cdots$ & 4.24 \\
\hline \multirow[t]{3}{*}{56} & 3.98 & 2 & 4.28 & 2 & 4.09 & 2 & 5.03 & $\ldots . .$. & $\ldots \ldots \ldots . . .$. \\
\hline & 4.52 & $\ldots \ldots$ & .............. & 9 & 4.56 & 7 & 5.26 & $\ldots . .$. & $\ldots \ldots \ldots \ldots$ \\
\hline & 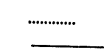 & $\ldots . .$. & 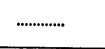 & 14 & 4.59 & $\ldots \ldots$ & 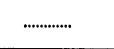 & $\ldots . .$. & .............. \\
\hline \multirow{3}{*}{$\begin{array}{l}\text { Mean } \\
\text { Mean for } \\
3 \text { dogs }\end{array}$} & 4.25 & $\ldots . \cdots$ & 4.28 & $\ldots . .$. & 4.41 & $\ldots . . . \cdot$ & 5.15 & $\ldots . . \cdot$ & .............. \\
\hline & & & & & & & & . & \\
\hline & 3.98 & $\ldots . .$. & 4.05 & $\ldots . .$. & .4 .01 & $\ldots . .$. & 4.70 & $\ldots . .$. & 4.22 \\
\hline
\end{tabular}

Table 46. Globulin in Plasma, in Grams per 100 Cubic Centimeters

\begin{tabular}{|c|c|c|c|c|c|c|c|c|c|}
\hline \multirow{2}{*}{$\begin{array}{l}\text { Dog } \\
\text { No. }\end{array}$} & \multirow{2}{*}{$\begin{array}{l}\text { Pre- } \\
\text { bleed- } \\
\text { ing } \\
\text { normal }\end{array}$} & \multicolumn{2}{|c|}{$\begin{array}{c}\text { After first } \\
\text { hemorrhage }\end{array}$} & \multicolumn{2}{|c|}{$\begin{array}{l}\text { After second } \\
\text { hemorrhage }\end{array}$} & \multicolumn{2}{|c|}{$\begin{array}{c}\text { After third } \\
\text { hemorrhage }\end{array}$} & \multicolumn{2}{|c|}{$\begin{array}{l}\text { After fourth } \\
\text { hemorrhage }\end{array}$} \\
\hline & & No. days & Amount & No. days & Amount & No. days & Amount & No. days & Amount \\
\hline \multicolumn{10}{|l|}{ Males } \\
\hline \multirow[t]{4}{*}{55} & 2.66 & 2 & 1.48 & 2 & 2.03 & 2 & 2.22 & 3 & 2.11 \\
\hline & 2.22 & $\ldots . .$. & ............... & 7 & 1.60 & 10 & 1.95 & 10 & 2.51 \\
\hline & $\ldots \ldots \cdots \cdots \cdots \cdot \cdots$ & $\ldots . .$. & 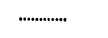 & 14 & 2.04 & 17 & 2.41 & $\ldots . .$. & $\ldots . . . \cdots \cdot \ldots$ \\
\hline & 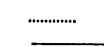 & $\ldots . .$. & .............. & $\ldots . .$. & $\ldots$ & 24 & 2.42 & $\ldots \ldots$. & ...................... \\
\hline Mean & 2.44 & $\ldots . .$. & 1.48 & $\ldots . .$. & 1.89 & $\ldots . .$. & 2.25 & $\ldots . .$. & 2.31 \\
\hline \multirow[t]{3}{*}{54} & 1.84 & 2 & 2.21 & 2 & 2.50 & 3 & 2.02 & 2 & 2.72 \\
\hline & $\ldots . . . \cdots \cdots \cdots$ & $\ldots . .$. & $\ldots$ & 9 & 2.08 & 10 & 2.44 & 8 & 2.69 \\
\hline & $\ldots+\cdots \cdots$ & $\ldots . . \cdot$ & $\ldots+\cdots \cdot \cdots \cdot \cdots$ & 16 & 1.97 & $\ldots . \cdot$. & $\ldots+\cdots \cdots$ & $\ldots . .$. & $\ldots$ \\
\hline Mean & 1.84 & $\ldots . . . \cdot$ & 2.21 & $\ldots . .$. & 2.18 & ....... & 2.23 & $\ldots . .$. & 2.70 \\
\hline \multirow[t]{3}{*}{56} & 2.83 & 2 & 1.98 & 2 & 2.29 & 2 & 1.17 & $\ldots . .$. & $\ldots . . . \cdots+\cdots$ \\
\hline & 3.36 & $\ldots \ldots$ & $\ldots+\ldots \ldots . . .$. & 9 & 2.80 & 7 & 1.85 & $\ldots . .$. & $\ldots . . . . . . . .$. \\
\hline & 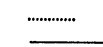 & $\ldots . \cdots$ & ...................... & 14 & 2.22 & $\ldots \cdots$ & $\ldots . . \cdots \cdots \cdots$ & $\ldots . \cdot \cdot$ & $\ldots$ \\
\hline Mean & 3.09 & $\ldots . .$. & 1.98 & $\ldots . .$. & 2.44 & $\ldots . .$. & 1.51 & $\ldots . .$. & .............. \\
\hline \multicolumn{10}{|l|}{ Mean for } \\
\hline 3 dogs & 2.46 & $\ldots . .$. & 1.89 & $\ldots . . . \cdot$ & 2.17 & $\ldots . . .$. & 2.00 & $\ldots . .$. & 2.51 \\
\hline
\end{tabular}


Table 47. Albumin-Globulin Ratio in Plasma

\begin{tabular}{|c|c|c|c|c|c|c|c|c|c|}
\hline \multirow{2}{*}{$\begin{array}{l}\text { Dog } \\
\text { No. }\end{array}$} & \multirow{2}{*}{$\begin{array}{l}\text { Pre- } \\
\text { bleed- } \\
\text { ing } \\
\text { normal }\end{array}$} & \multicolumn{2}{|c|}{$\begin{array}{c}\text { After first } \\
\text { hemorrhage }\end{array}$} & \multicolumn{2}{|c|}{$\begin{array}{l}\text { After second } \\
\text { hemorrhage }\end{array}$} & \multicolumn{2}{|c|}{$\begin{array}{c}\text { After third } \\
\text { hemorrhage }\end{array}$} & \multicolumn{2}{|c|}{$\begin{array}{l}\text { After fourth } \\
\text { hemorrhage }\end{array}$} \\
\hline & & No. days & Amount & No. days & Amount & No. days & Amount & No. days $P$ & Amount \\
\hline \multicolumn{10}{|l|}{ Males } \\
\hline \multirow[t]{4}{*}{55} & 1.42 & 2 & 2.68 & 2 & 1.47 & 2 & 2.10 & 3 & 1.93 \\
\hline & 1.62 & $\ldots . .$. & .............. & 7 & 2.31 & 10 & 2.27 & 10 & 1.72 \\
\hline & $\ldots$ & ....... & 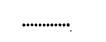 & 14 & 2.05 & 17 & 1.71 & $\ldots . .$. & $\ldots$ \\
\hline & $\ldots$ & $\ldots . .$. & $\ldots$ & $\ldots . .$. & $\ldots \ldots$ & 24 & 1.85 & $\ldots . .$. & $\ldots \ldots$ \\
\hline Mean & 1.52 & $\ldots . .$. & 2.68 & $\ldots . .$. & 1.94 & $\ldots . .$. & 1.98 & $\ldots . .$. & 1.83 \\
\hline \multirow[t]{3}{*}{54} & 2.18 & 2 & 1.76 & 2 & 1.44 & 3 & 2.29 & 2 & 1.49 \\
\hline & $\ldots+. . . . . .$. & $\ldots . .$. & $\ldots . . . \cdots \cdots$ & 9 & 1.99 & 10 & 1.81 & 8 & 1.64 \\
\hline & $\ldots$ & $\ldots . .$. & ............... & 16 & 2.15 & $\ldots . . \cdot$ & $\ldots+\cdots \cdot \cdots$ & $\ldots . .$. & $\ldots$ \\
\hline Mean & 2.18 & $\ldots . .$. & 1.76 & $\ldots . .$. & 1.86 & $\ldots . .$. & 2.05 & $\ldots . .$. & 1.57 \\
\hline \multirow[t]{3}{*}{56} & 1.41 & 2 & 2.16 & 2 & 1.79 & 2 & 4.30 & $\ldots . .$. & $\ldots \ldots . . . .$. \\
\hline & 1.34 & $\ldots \ldots$ & 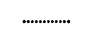 & 9 & 1.63 & 7 & 2.84 & $\ldots . .$. & $\ldots \ldots \ldots . .$. \\
\hline & .............. & $\ldots . \cdot$ & 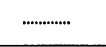 & 14 & 2.07 & $\ldots . .$. & 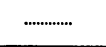 & $\ldots . .$. & $\ldots \ldots \ldots$ \\
\hline Mean & 1.37 & $\ldots . .$. & 2.16 & $\ldots . .$. & 1.83 & $\ldots . .$. & 3.57 & $\cdots \cdot$. & $\ldots \ldots \ldots$ \\
\hline \multicolumn{10}{|l|}{ Mean for } \\
\hline 3 dogs & 1.69 & $\ldots . .$. & 2.20 & $\ldots . .$. & 1.88 & $\ldots . .$. & 2.53 & $\ldots . .$. & 1.70 \\
\hline
\end{tabular}

Table 48. Osmotic Pressure of Plasma Proteins, in Millimeters of Mercury (Calculated on Basis of Govaerts' Factors)

\begin{tabular}{|c|c|c|c|c|c|c|c|c|c|}
\hline \multirow{2}{*}{$\begin{array}{l}\text { Dog } \\
\text { No. }\end{array}$} & \multirow{2}{*}{$\begin{array}{l}\text { Pre- } \\
\text { bleed- } \\
\text { ing } \\
\text { normal }\end{array}$} & \multicolumn{2}{|c|}{$\begin{array}{c}\text { After first } \\
\text { hemorrhage }\end{array}$} & \multicolumn{2}{|c|}{$\begin{array}{l}\text { After second } \\
\text { hemorrhage }\end{array}$} & \multicolumn{2}{|c|}{$\begin{array}{c}\text { After third } \\
\text { hemorrhage }\end{array}$} & \multicolumn{2}{|c|}{$\begin{array}{l}\text { After fourth } \\
\text { hemorrhage }\end{array}$} \\
\hline & & No. days & Amount & No. days & Amount & No. days & $\overline{\text { Amount }}$ & No. days & Amount \\
\hline \multicolumn{10}{|l|}{ Males } \\
\hline \multirow[t]{4}{*}{55} & 24.4 & 2 & 23.8 & 2 & 19.2 & 2 & 28.6 & 3 & 25.3 \\
\hline & 22.8 & $\ldots . .$. & $\ldots . . . . . . .$. & 7 & 22.5 & 10 & 27.0 & 10 & 27.2 \\
\hline & $\ldots . . . . . . . . .$. & $\ldots . .$. & $\ldots . . . . . . .$. & 14 & 25.8 & 17 & 25.9 & $\ldots . .$. & $\ldots$ \\
\hline & $\ldots . . . \cdots \cdot . .$. & $\ldots . .$. & ............... & $\ldots . .$. & $\ldots-\cdots \cdot \cdots \cdot \cdot \cdot$ & 24 & 27.9 & $\ldots . .$. & $\ldots$ \\
\hline Mean & 23.6 & $\ldots . . .$. & 23.8 & $\ldots . . .$. & 22.5 & $\ldots . . .$. & 27.4 & $\ldots . . .$. & 26.2 \\
\hline \multirow[t]{3}{*}{54} & 24.7 & 2 & 24.4 & 2 & 23.2 & 3 & 28.1 & 2 & 26.0 \\
\hline & $\ldots . . . . . . .$. & $\ldots . .$. & $\ldots \ldots \ldots . . . .$. & 9 & 25.6 & 10 & 27.6 & 8 & 28.0 \\
\hline & $\ldots . . . \cdot \cdots \cdot \cdot$ & $\ldots . .$. & 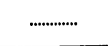 & 16 & 26.5 & ...... & $\ldots$ & $\ldots . .$. & $\ldots . . \cdots \cdots \cdots$ \\
\hline Mean & 24.7 & $\ldots . .$. & 24.4 & $\ldots . . .$. & 25.1 & $\ldots . .$. & 27.8 & $\ldots . .$. & 27.0 \\
\hline \multirow[t]{3}{*}{56} & 25.8 & 2 & 26.2 & 2 & 25.6 & 2 & 29.2 & ....... & ................... \\
\hline & 29.5 & $\ldots . .$. & $\ldots . . . \cdots \cdots \cdot . .$. & 9 & 28.9 & 7 & 31.4 & ....... & $\ldots \ldots$ \\
\hline & ............... & $\ldots . .$. & $\ldots$ & 14 & 28.2 & $\ldots . .$. & $\ldots . . \cdots \cdots \cdot \cdots$ & $\ldots . .$. & $\ldots$ \\
\hline Mean & 27.6 & $\ldots . .$. & 26.2 & $\ldots . . .$. & 27.6 & $\ldots . .$. & 30.3 & $\ldots . .$. & $\ldots$ \\
\hline \multicolumn{10}{|c|}{ Mean for } \\
\hline 3 dogs & 25.3 & $\ldots . .$. & 24.8 & $\ldots . .$. & 25.1 & $\ldots . .$. & 28.5 & $\ldots . .$. & 26.6 \\
\hline
\end{tabular}


Since the plasma proteins contribute to the maintenance of the osmotic pressure of the blood, it was interesting to note the extent to which changes in the protein levels influenced the change in osmotic pressure. Govaerts (1925, 1926) had suggested factors to be used in determining the osmotic pressure contributed by the plasma proteins. Based on these factors, the calculated mean normal osmotic pressure contributed by the albumin and globulin was 25.3 millimeters of mercury (table 48).

Following the first hemorrhage, the mean was slightly below normal, 24.8 millimeters, but after the second hemorrhage it increased slightly

Table 49. Fibrinogen in Plasma, in Grams per 100 Cubic Centimeters

\begin{tabular}{|c|c|c|c|c|c|c|c|c|c|}
\hline \multirow{2}{*}{$\begin{array}{l}\text { Dog } \\
\text { No. }\end{array}$} & \multirow{2}{*}{$\begin{array}{l}\text { Pre- } \\
\text { bleed- } \\
\text { ing } \\
\text { normal. }\end{array}$} & \multicolumn{2}{|c|}{$\begin{array}{c}\text { After first } \\
\text { hemorrhage }\end{array}$} & \multicolumn{2}{|c|}{$\begin{array}{l}\text { After second } \\
\text { hemorrhage }\end{array}$} & \multicolumn{2}{|c|}{$\begin{array}{c}\text { After third } \\
\text { hemorrhage }\end{array}$} & \multicolumn{2}{|c|}{$\begin{array}{l}\text { After fourth } \\
\text { hemorrhage }\end{array}$} \\
\hline & & No. days & Amount & No. days & Amount & No. days & Amount & No. days & Āmount \\
\hline \multicolumn{10}{|l|}{ Males } \\
\hline \multirow[t]{4}{*}{55} & 0.11 & 2 & 0.17 & 2 & 0.24 & 2 & 0.28 & 3 & 0.28 \\
\hline & 0.23 & $\ldots . .$. & $\ldots$ & 7 & 0.26 & 10 & 0.35 & 10 & 0.23 \\
\hline & $\ldots \ldots \ldots \ldots$ & $\ldots . .$. & $\ldots-\ldots \ldots . . .$. & 14 & 0.25 & 17 & 0.38 & $\ldots . .$. & $\ldots \ldots \ldots \ldots . .$. \\
\hline & $\ldots \ldots \ldots \ldots$ & $\ldots . .$. & $\ldots . . . \cdots \cdot \cdots$ & $\ldots . .$. & $\ldots$ & 24 & 0.28 & $\ldots . \cdot$. & ...................... \\
\hline Mean & 0.17 & $\ldots . .$. & 0.17 & $\ldots . . .$. & 0.25 & $\ldots . .$. & 0.32 & $\ldots . .$. & 0.26 \\
\hline \multirow[t]{3}{*}{54} & 0.33 & 2 & 0.34 & 2 & 0.27 & 3 & 0.30 & 2 & 0.33 \\
\hline & $\ldots \ldots \ldots$ & $\ldots . .$. & 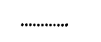 & 9 & 0.35 & 10 & 0.33 & 8 & 0.30 \\
\hline & 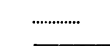 & $\ldots . .$. & $\ldots$ & 16 & 0.33 & $\ldots . .$. & $\ldots \ldots \ldots$ & $\ldots . .$. & 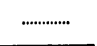 \\
\hline Mean & 0.33 & $\ldots . .$. & 0.34 & $\ldots . .$. & 0.32 & $\ldots . . .$. & 0.32 & $\ldots . .$. & 0.32 \\
\hline \multirow[t]{3}{*}{56} & 0.25 & 2 & 0.20 & 2 & 0.24 & 2 & 0.23 & $\ldots . .$. & $\ldots \ldots \ldots$ \\
\hline & 0.31 & $\ldots . .$. & $\ldots$ & 9 & 0.23 & 7 & 0.23 & $\ldots . \cdots$ & $\ldots \ldots \ldots$ \\
\hline & $\ldots \ldots \cdots \cdots \cdots$ & $\ldots . .$. & .................. & 14 & 0.20 & $\ldots . .$. & ............... & $\ldots . .$. & 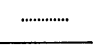 \\
\hline Mean & 0.28 & $\ldots . .$. & 0.20 & $\ldots . .$. & 0.22 & $\ldots . .$. & 0.23 & $\ldots \ldots$ & 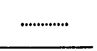 \\
\hline \multicolumn{10}{|l|}{ Mean for } \\
\hline 3 dogs & 0.26 & $\ldots \ldots$ & 0.24 & $\ldots . .$. & 0.26 & $\ldots . .$. & 0.29 & $\ldots . .$. & 0.29 \\
\hline
\end{tabular}

to a level of 25.1 millimeters, the latter value representing a mean of three determinations on each of the three animals. Calculation of the mean for the first determination on each of the three dogs gave a value of 22.7 millimeters. Similar calculations for the second and third determinations produced means of 25.7 and 26.8 millimeters, respectively, showing a gradual rise in osmotic pressure during the period following the second hemorrhage.

Following the third hemorrhage, the osmotic pressure contributed by the plasma proteins was decidedly above normal, 28.5 millimeters of mercury. This value also represented a mean of two to four cleterminations on each of the three animals. The mean of the first determinations was 28.6 millimeters of mercury; of the second, 28.7. It is evident, therefore, that there was no apparent increase in osmotic pressure between the first and second determinations after the third hemorrhage. 
However, an increase could hardly have been anticipated in view of the high values observed in the first determinations. In the two dogs subjected to the fourth bleeding, a value noticeably above normal, 26.6 millimeters, but lower than the value observed after the third hemorrhage, was noted. This value was based on two determinations for each animal.

Fibrinogen.-It is evident from values obtained that fibrinogen lost through hemorrhage is replaced rapidly. It may be noted from table 49 that the normal fibrinogen level was $0.26 \mathrm{~g}$. per $100 \mathrm{cc}$. of plasma. The means observed following the first, second, third, and fourth hemorrhages were respectively $0.24,0.26,0.29$, and $0.29 \mathrm{~g}$. per $100 \mathrm{cc}$. of plasma.

\section{DISCUSSION}

\section{Plasma Volume}

Robertson and Bock's (1919) observations on wounded soldiers showed that the blood volume was restored relatively slowly after hemorrhage. This condition, they suggested, might be due to an insufficient quantity of reserve fluids in the tissue since, if the patients were given large quantities of fluid by alimentary tract, rapid increases in blood volume resulted and were maintained. This need for fluids was substantiated by the fact that the animals used in the present investigation evidenced thirst immediately after hemorrhage.

Ludwig (1931) postulated the existence of "plasma depots" similar to the "cell depots" which have been reported as existing in the body. He suggested the liver as the primary source of this reserve plasma. That a certain amount of reserve plasma exists in the body is apparent from the alterations noted in cell volume per cent within 30 minutes of the start of hemorrhage (during the short-term study) and before the animals had been permitted to ingest any water. Restoration of plasma volume was usually complete, and in most cases actually above the normal level, within 24 hours after bleeding.

The animals subjected to two bleedings, in the long-term study, in most instances showed plasma volumes appreciably above the normal level before the end of the first post-bleeding week.

\section{Blood Plasma Constituents}

The rapid recovery in the osmotic pressure of the blood, specifically the recovery in plasma proteins and the various calcium and phosphorus fractions, may partially account for the recovery in plasma volume. One factor that may contribute to the increase in the osmotic pressure of the blood is the nonprotein nitrogen. A number of investigators have reported increases in the nonprotein nitrogen level following hemorrhage 
(Taylor and Lewis, 1915a ; Kerr, Hurwitz, and Whipple, 1918-19; Buell, 1919; Schlutz, Swanson, and Ziegler, 1928). In general, the changes noted would appear to be transitory, lasting from only a few hours to two or three days.

In the short-term study, the nonprotein nitrogen level per unit of volume was slightly below normal during the post-bleeding period. However, the total amount in the circulating plasma after hemorrhage was in remarkable agreement with the observed pre-bleeding total. It is possible that the total amount in the circulation is of greater significance than the level in the blood. This is also true of the various calcium and phosphorus fractions (table 38).

Opinions differ as to the effect of hemorrhage on blood phosphorus. Malan (1928) found that repeated hemorrhage in sheep resulted in a lowering of total and lipide phosphorus, while the inorganic fraction showed no consistent variation. Contrary to these findings, Fishberg (1929) noted a rise in inorganic phosphorus following repeated bleeding of rabbits. Nitzescu and Runceanu (1927) reported that inorganic phosphorus rose slowly in dogs, and only in the last stage of a fatal hemorrhage. Youngburg and Youngburg (1936), using rats as experimental animals, observed "no change or, possibly, a decrease in the phosphorus compounds" which they studied-inorganic, organic acid soluble, and lipide phosphorus.

In the short-term study, values slightly below normal were observed for both lipide and inorganic phosphorus throughout the post-bleeding period. On the basis of the total amount in the circulating plasma, however, (table 38) the post-bleeding means exceeded slightly the prebleeding means, again suggesting, as in the case of protein and nonprotein nitrogen, that the total amount in the circulation is more significant than the level.

There was also a divergence of opinion regarding the effect of hemorrhage on the calcium level. Kauftheil and Kisch (1927b), working with rabbits rendered anemic through bleeding, found that blood calcium varied only slightly with the degree of anemia. On the other hand, Fishberg (1929), reporting a similar experiment on rabbits, noted a fall in serum calcium in severe hemorrhagic anemia. Sekitoo (1929-30) stated that repeated bleeding caused a slight hypocalcemia which, however, disappeared soon after the bleeding stopped, the calcium level returning quickly to normal. Kapsinow and Underhill (1929) noted a similar fall in blood calcium, owing to bleeding; Charles (1931-32) reported a rise ; and Culhane (1930) and Mirvish and Bosman (1927-28) found no effect on the calcium level. Nitzescu and Runceanu (1927) observed in dogs and rabbits a marked rise in calcium immediately before death resulting from bleeding. Kauftheil and Kisch (1927a) reported that human blood calcium is almost always below normal in anemia. 
In the short-term study, values that were slightly below normal were observed following hemorrhage for the diffusible calcium whereas nondiffusible calcium, 24 hours after bleeding, showed a mean level slightly above normal, values slightly below the normal being observed thereafter. As in the case of the phosphorus fractions, the total amount of the calcium fractions in the circulating plasma displayed, during the postbleeding period, mean values that were somewhat above the pre-bleeding means, again suggesting the greater importance of the total quantity in the circulation than of the level.

Although some investigators (Peters and Eiserson, 1929, and Greenwald, 1931, working with human subjects, and Darrow and Cary, 1934, working with dogs) have reported relationships between protein and calcium of the plasma, no significant correlations were obtained in the present investigation, which was in accord with the report of Palmer, Gortner, and Rude (1930) covering their work with cattle.

There have been a number of excellent studies on the regeneration of plasma proteins following depletion. In some instances, the studies were based on hemorrhages in which a measured amount of the total blood volume was removed. In others, the plasmapheresis technique was employed. In this latter type of experiment, plasma protein regeneration was followed over a considerable period of time, using a variety of test foods. On the basis of these studies, potency ratios for various proteins were established (Pommerenke, Slavin, Kariher, and Whipple, 1935 ; Holman, Mahoney, and Whipple, 1934; McNaught, Scott, Woods, and Whipple, 1936; Madden, Winslow, Howland, and Whipple, 1937 ; Melnick, Cowgill, and Burack, 1936; and Melnick and Cowgill, 1937).

In the current research, in which plasma protein regeneration was observed in animals subjected to repeated bleedings, recovery of the albumin fraction occurred with great rapidity whereas globulin recovery was accomplished with difficulty. This observation agreed with those reported by Morawitz (1906) and Cuvelier and Patoir (1932), but not with those of Kerr, Hurwitz, and Whipple (1918-19) and Schlutz, Swanson, and Ziegler (1928), who reported a more rapid recovery of the globulin fraction following hemorrhage. Since Pommerenke, Slavin, Kariher, and Whipple have reported that plant proteins apparently favor the production of globulin, while animal proteins favor the regeneration of albumin, it is possible that the differences reported were dependent upon the experimental diet employed. In the present investigation, casein was used as the sole protein in the diet, which should and did favor the regeneration of albumin.

Whipple, Smith, and Belt (1920) postulated that the liver was involved in the regeneration of new plasma protein but suggested at the same time that other organs might be able in an emergency to function also in this capacity. Based on in vitro experiments, Bellis and Scott 
(1935) reported that a considerable part of the plasma protein was derived from proteins adsorbed on the red corpuscles, released after hemorrhage through dilution of the plasma. According to these investigators, the increase in protein following dilution was due chiefly to albumin, although euglobulin (fibrinogen in oxalated blood) and pseudoglobulin I also were increased to some extent.

Apparently there was agreement among workers concerning the celerity with which the level of fibrinogen is restored in the plasma. Whipple, Smith, and Belt found that replacement of this protein occurred rapidly, the normal level being regained within 24 hours after hemorrhage. This confirmed the earlier work of Goodpasture (1914), who after having reduced the fibrinogen in the blood to a level where its presence could not be detected noted appreciable increases in this protein fraction within 15 minutes, followed within another 15 minutes by sufficient clotting to permit inversion of the tube. In the present investigation, in the animals that were bled three to four times, it was also observed that fibrinogen regeneration occurred very quickly. In general, the post-bleeding values were quite similar to the pre-bleeding normal levels.

\section{SUMMARY \\ Plasma Volume}

In the short-term study, within 30 minutes of the start of hemorrhage, in which 20 per cent of the total blood volume was removed, plasma volume had recovered to within 13 per cent of the pre-bleeding mean. Twenty-four hours later it had recovered to a level slightly above the pre-bleeding mean. On succeeding days further slight increases occurred to compensate for losses in cell volume owing to sampling. In the long-term study, for the females plasma volume per kilogram was appreciably above the pre-bleeding mean one week after hemorrhage, with gradual decreases thereafter to a level approaching the pre-bleeding normal six weeks after hemorrhage. For the males, plasma volume per kilogram was also considerably above the pre-bleeding mean one week after hemorrhage. Thereafter gradual decreases resulted in a mean slightly above the pre-bleeding level six weeks after hemorrhage. Little alteration was noted for either males or females throughout the remainder of the post-bleeding period.

\section{Blood Plasma Constituents}

In the short-term study, both the protein nitrogen and nonprotein nitrogen levels were somewhat below the pre-bleeding means throughout the post-bleeding period. The mean of the total protein nitrogen in the circulating plasma after hemorrhage was slightly greater than the pre- 
bleeding mean, while the mean of the total amount of nonprotein nitrogen after hemorrhage was identical with the pre-bleeding value.

The lipide and inorganic phosphorus levels were somewhat below the pre-bleeding means throughout the post-bleeding period, which condition was reflected in the total phosphorus level. For both phosphorus fractions, the mean total amount in the circulating plasma after hemorrhage was slightly greater than the pre-bleeding mean.

The level of diffusible calcium was slightly below the pre-bleeding mean throughout the post-bleeding period. For nondiffusible calcium, the mean level 24 hours after bleeding was somewhat higher than the pre-bleeding mean, but values below the pre-bleeding mean were observed throughout the remainder of the post-bleeding period. The mean total amount of the calcium fractions in the circulating plasma after hemorrhage was slightly greater than the pre-bleeding mean.

Significant positive correlations were obtained between the levels of plasma protein and inorganic phosphorus before hemorrhage, +.3248 , and after hemorrhage, +.3487.

Significant positive correlations were found, after hemorrhage, between lipide phosphorus and nonprotein nitrogen levels, +.2575 ; between lipide phosphorus and inorganic phosphorus, +.4371 ; between lipide phosphorus and diffusible calcium, +.3077 ; and between inorganic phosphorus and diffusible calcium, +.3897 . No such relationships were apparent in pre-bleeding levels.

A significant negative correlation was obtained between pre-bleeding levels for diffusible and nondiffusible calcium, - .3787 , but not between post-bleeding values.

In the fourth study, in which the dogs were bled three or four times, the mean albumin level returned promptly to the pre-bleeding normal following each unit hemorrhage, attaining a level somewhat above normal after the third and fourth hemorrhages. The globulin level failed to return to the pre-bleeding normal after the first three unit hemorrhages, although apparently there was increasing ability to regenerate globulin as shown by increased means following each unit hemorrhage. Alterations in the levels of albumin and globulin were reflected in the albumin-globulin ratio. Calculation of the osmotic pressure contributed by the albumin and globulin showed a prompt return to the pre-bleeding normal, with values above normal after the third and fourth hemorrhages.

The fibrinogen level recovered promptly following each unit hemorrhage, the means obtained after the third and fourth hemorrhages being slightly above the pre-bleeding mean. 


\section{WHOLE BLOOD \\ TOTAL BLOOD VOLUME}

A S POINTED OUT in section IV on blood cells, the failure of the total blood volume to return to normal following hemorrhage leads to an erroneous conception of the degree of anemia induced by bleeding when red cell count, oxygen-combining capacity, or cell volume per cent is used as the criterion for diagnosing the condition of an anemic patient.

The mean pre-bleeding total volume for the animals used in the shortterm study was $75.5 \mathrm{cc}$. per kilogram (table 50). Within 30 minutes after the start of bleeding the total blood volume had decreased 17 per cent, to a mean of $62.9 \mathrm{cc}$. per kilogram. The rapid restoration of plasma explains why this decrease did not more nearly approximate the percentage of the blood removed. On the day following hemorrhage, the mean was $67.8 \mathrm{cc}$. per kilogram, followed by slightly lower values throughout the remainder of the post-bleeding period.

Table 50. Values for Total Blood Volume, in Cubic Centimeters per Kilogram (Short-term study)

\begin{tabular}{|c|c|c|c|c|c|c|}
\hline \multirow{2}{*}{$\begin{array}{c}\text { Dog } \\
\text { No. }\end{array}$} & \multirow{2}{*}{$\begin{array}{l}\text { Last pre- } \\
\text { bleeding }\end{array}$} & \multirow{2}{*}{$\begin{array}{l}\text { Within } \\
30 \text { minutes } \\
\text { of start of } \\
\text { bleeding }\end{array}$} & \multicolumn{4}{|c|}{ Post-bleeding period } \\
\hline & & & 1 day & 2 days & 3 days & 5.7 days \\
\hline \multicolumn{7}{|l|}{ Males } \\
\hline \multirow[t]{2}{*}{44} & 75.8 & 62.5 & 67.8 & 66.1 & 62.7 & 62.8 \\
\hline & 74.6 & 56.8 & 65.9 & 65.5 & 63.7 & 67.7 \\
\hline \multirow[t]{2}{*}{45} & 78.4 & $\ldots+\ldots \ldots . . . .$. & 69.7 & 70.4 & 69.2 & 67.3 \\
\hline & 77.5 & 58.5 & 73.5 & 65.0 & 66.5 & 65.7 \\
\hline \multirow[t]{2}{*}{46} & 81.3 & 74.0 & 77.1 & 72.6 & 76.4 & 71.0 \\
\hline & 80.8 & 68.9 & 65.3 & 69.7 & 68.6 & 66.7 \\
\hline \multirow[t]{4}{*}{47} & 73.4 & 66.7 & 66.4 & 56.8 & 55.9 & 67.5 \\
\hline & 74.3 & 65.2 & 63.4 & 67.2 & 67.4 & 68.1 \\
\hline & 71.1 & 55.7 & 58.2 & 60.5 & 58.7 & 57.6 \\
\hline & 67.8 & 57.8 & 70.1 & 62.6 & 66.5 & 65.6 \\
\hline Mean & 75.5 & 62.9 & 67.8 & 65.6 & 65.6 & 66.0 \\
\hline
\end{tabular}

In the long-term study the pre-bleeding mean for the females was $67.6 \mathrm{cc}$. per kilogram (table 51). One week following hemorrhage, the mean value was 59.0 cc., where it remained practically constant throughout the first six weeks of the post-bleeding period. It will be recalled that these animals made practically no progress in restoring total cell volume (table 20), which condition was reflected in the failure of the total blood volume to recover throughout this six-week interval. The mean value noted for the females observed for 12 weeks, 56.8 cc., was 
almost identical with that observed for these animals at the end of the sixth week, $56.9 \mathrm{cc}$. per kilogram. Following the initial adjustment to blood loss by increase in plasma, further adjustment was evidently dependent upon the ability or inability of the animal to restore cell volume in that the animals that were most capable of cell volume regeneration

Table 51. Values for Total Blood Volume, in Cubic Centimeters per Kilogram (Long-term study)

\begin{tabular}{|c|c|c|c|c|c|c|c|c|c|}
\hline \multirow{2}{*}{$\begin{array}{l}\text { Dog } \\
\text { No. }\end{array}$} & \multirow{2}{*}{$\begin{array}{c}\text { Pre- } \\
\text { bleeding } \\
\text { normal }\end{array}$} & \multicolumn{8}{|c|}{ Post-bleeding period } \\
\hline & & 1-5 days & $1 \mathrm{wk}$. & 2 wks. & 3 wks. & 4 wks. & 5 wks. & 6 wks. & 12 wks. \\
\hline \multicolumn{10}{|c|}{ Females } \\
\hline 20 & 68.6 & 59.8 & 64.2 & 56.1 & 64.6 & 59.5 & 59.8 & 64.0 & 65.9 \\
\hline 21 & 68.5 & 69.4 & 63.8 & 66.3 & 55.4 & 62.2 & 60.9 & 63.4 & ............... \\
\hline 22 & 61.8 & 59.4 & 50.7 & 58.3 & 54.6 & 57.1 & 61.0 & 60.7 & $\ldots \ldots . . . . . .$. \\
\hline 19 & 70.2 & 64.4 & 56.1 & 56.3 & $\ldots \ldots \ldots . . .$. & 59.7 & 52.6 & 50.8 & 47.1 \\
\hline 32 & 69.0 & 56.6 & 60.1 & 57.6 & 57.1 & 59.0 & 57.1 & 56.4 & 57.5 \\
\hline Mean & 67.6 & 61.9 & 59.0 & 58.9 & 57.9 & 59.5 & 58.3 & 59.1 & 56.8 \\
\hline \multicolumn{10}{|l|}{ Males } \\
\hline 17 . & 69.8 & 59.1 & 64.0 & 64.6 & 69.1 & 61.7 & 58.5 & 65.4 & 70.6 \\
\hline 18 & 58.4 & 54.9 & 50.0 & 48.5 & 56.8 & 60.3 & 54.2 & 53.2 & 56.1 \\
\hline 19 & 75.4 & 72.0 & 73.3 & 78.1 & 78.8 & 75.4 & 85.6 & 80.0 & 74.8 \\
\hline 31 & 71.4 & 62.0 & 69.3 & 70.6 & 70.6 & 72.0 & 75.3 & 71.1 & 70.6 \\
\hline Mean & 68.8 & 62.0 & 64.1 & 65.4 & 68.8 & 67.4 & 68.4 & 67.4 & 68.0 \\
\hline
\end{tabular}

also most nearly approached their normal total blood volume. This is further evidenced by the data secured on the males in this study in that these animals displayed a greater capacity for cell volume regeneration than did the females, and therefore also a greater recovery in total blood volume. The mean pre-bleeding value for the males was $68.8 \mathrm{cc}$. per kilogram. One week after hemorrhage, a value of $64.1 \mathrm{cc}$. was observed. Total blood volume recovered to the normal level by the third week and continued at practically the same level throughout the rest of the postbleeding period.

\section{CONSTITUENTS OF WHOLE BLOOD}

Protcin Nitrogen.-The data presented in this section were secured in the long-term study. The pre-bleeding mean value for protein nitrogen of whole blood for the five females used in this study was $3.08 \mathrm{~g}$. per $100 \mathrm{cc}$. (table 52). One week after hemorrhage a value of $2.56 \mathrm{~g}$. was obtained, with a further reduction to $2.44 \mathrm{~g}$. at the end of the sixth postbleeding week, representing 79 per cent of the normal. For the two females observed for 12 weeks, the protein nitrogen level was found to be $2.44 \mathrm{~g}$., contrasted with a value of $2.19 \mathrm{~g}$. observed for these animals 
at the end of the sixth week, indicating a very slight increase during the second six-week portion of the post-bleeding period. Since approximately two thirds of the nitrogen of the blood is found in the red blood cells, the failure of the experimental animals to recover their cell volume

Table 52. Values for Total Protein Nitrogen in Blood, in Grams per 100 Cubic Centimeters

(Long-term study)

\begin{tabular}{|c|c|c|c|c|c|c|c|c|c|}
\hline \multirow{2}{*}{$\begin{array}{l}\text { Dog } \\
\text { No. }\end{array}$} & \multirow{2}{*}{$\begin{array}{c}\text { Pre- } \\
\text { bleeding } \\
\text { normal }\end{array}$} & \multicolumn{8}{|c|}{ Post-bleeding period } \\
\hline & & 1-2 days & 1 wk. & 2 wks. & 3 wks. & 4 wks. & 5 wks. & 6 wks. & 12 wks. \\
\hline \multicolumn{10}{|c|}{ Females } \\
\hline 20 & 2.86 & 2.20 & 2.53 & 2.24 & 2.01 & 2.36 & 2.24 & 2.08 & 2.61 \\
\hline 21 & 3.14 & 2.48 & 2.76 & 2.46 & 2.66 & 2.74 & 2.60 & 2.59 & $\ldots . . . \cdots \cdots \cdot$ \\
\hline 22 & 2.81 & 2.27 & 2.30 & 2.44 & 2.52 & 2.56 & 2.69 & 2.67 & 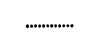 \\
\hline 19 & 3.44 & 2.62 & 2.77 & 2.64 & 2.47 & 2.67 & 2.65 & 2.30 & 2.28 \\
\hline 32 & 3.14 & 2.47 & 2.44 & 2.32 & 2.41 & 2.44 & 2.52 & 2.57 & $\ldots \ldots \ldots \ldots . .$. \\
\hline Mean & 3.08 & 2.41 & 2.56 & 2.42 & 2.41 & 2.55 & 2.54 & 2.44 & 2.44 \\
\hline \multicolumn{10}{|l|}{ Males } \\
\hline 17 & 3.33 & 2.52 & 2.44 & 2.84 & 2.66 & 2.95 & 2.74 & 2.76 & 2.72 \\
\hline 18 & 3.39 & 2.41 & 2.28 & 2.24 & 2.34 & 2.30 & 2.40 & 2.26 & 2.34 \\
\hline 30 & 2.94 & 2.36 & 2.12 & 2.22 & 2.82 & 2.94 & 3.05 & 2.79 & $\ldots \ldots \ldots \ldots . .$. \\
\hline 31 & 3.32 & 2.58 & 2.05 & 2.71 & 2.80 & 2.89 & 2.95 & 2.96 & 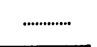 \\
\hline Mean & 3.24 & 2.47 & 2.22 & 2.50 & 2.66 & 2.77 & 2.78 & 2.69 & 2.53 \\
\hline
\end{tabular}

after hemorrhage readily explains the continued low total protein nitrogen in the blood.

The male dogs, it will be recalled, made considerably better progress than the females in recovering their normal cell volume, which tendency was again evidenced by their greater recovery in total protein nitrogen. The pre-bleeding normal level of total protein nitrogen was $3.24 \mathrm{~g}$. per 100 cubic centimeters. At the end of the first week after hemorrhage a value of $2.22 \mathrm{~g}$. per $100 \mathrm{cc}$. was obtained; at the end of the sixth week, $2.69 \mathrm{~g}$., the latter value representing 83 per cent of the pre-bleeding normal. By the end of the twelfth week, the total protein nitrogen level, based on two dogs, was $2.53 \mathrm{~g}$. per 100 cc., showing no change from the mean noted for these two animals at the end of the sixth week, 2.51 per 100 cubic centimeters.

Nonprotein Nitrogen.-The mean pre-bleeding nonprotein nitrogen level for both male and female animals was $32.4 \mathrm{mg}$. per $100 \mathrm{cc}$. of blood (table 53). At the end of the first post-bleeding week, the mean for the females was 36.6 milligrams; for the males, 33.2 milligrams; with values of $34.3 \mathrm{mg}$. and $31.0 \mathrm{mg}$. for the females and male, respectively, at the end of the sixth post-bleeding week. For the two females observed for 12 weeks, the level noted at the end of the period was $30.0 \mathrm{mg}$., contrasted with $31.8 \mathrm{mg}$. for these same animals at the end of the sixth 
week. For the males, the value (based on two animals) observed at the end of the twelfth week was $26.4 \mathrm{mg}$., which value was in close agreement with the mean for these same animals at the end of the sixth week, 27.0 milligrams.

Table 53. Values for Nonprotein Nitrogen in Blood, in Milligrams per 100 Cubic Centimeters

(Long-term study)

\begin{tabular}{|c|c|c|c|c|c|c|c|c|c|}
\hline \multirow{2}{*}{$\begin{array}{l}\text { Dog } \\
\text { No. }\end{array}$} & \multirow{2}{*}{$\begin{array}{c}\text { Pre- } \\
\text { bleeding } \\
\text { normal }\end{array}$} & \multicolumn{8}{|c|}{ Post-bleeding period } \\
\hline & & 1-2 days & 1 wk. & 2 wks. & 3 wks. & 4 wks. & 5 wks. & 6 wks. & 12 wks. \\
\hline \multicolumn{10}{|c|}{ Females } \\
\hline 20 & 28.0 & 35.1 & 32.5 & 34.5 & 28.3 & 31.1 & 27.9 & 28.5 & 31.5 \\
\hline 21 & 25.5 & 34.7 & 32.5 & 27.7 & 37.1 & 29.7 & 26.0 & 32.1 & $\ldots \ldots \ldots \ldots$ \\
\hline 22 & 38.9 & 53.3 & 51.5 & 39.5 & 34.5 & 37.9 & 46.9 & 41.5 & $\ldots$ \\
\hline 19 & 37.3 & 32.6 & 35.6 & 33.0 & 31.2 & 38.0 & 31.0 & 35.2 & 28.6 \\
\hline 32 & 32.4 & 35.8 & 30.9 & 32.7 & 32.0 & 34.4 & 30.0 & 34.4 & $\ldots \ldots \ldots \ldots$ \\
\hline Mean & 32.4 & 38.1 & 36.6 & 33.5 & 32.6 & 34.2 & 32.4 & 34.3 & 30.0 \\
\hline \multicolumn{10}{|l|}{ Males } \\
\hline 17 & 30.1 & 26.7 & 24.2 & 26.8 & $27.6^{\circ}$ & 26.3 & 27.2 & 25.8 & 29.1 \\
\hline 18 & 29.9 & 28.0 & 30.2 & 29.1 & 28.9 & 27.1 & 28.8 & 28.2 & 23.8 \\
\hline 30 & 35.1 & 32.5 & 39.1 & 38.3 & 35.8 & 34.2 & 32.8 & 32.0 & $\ldots \ldots \ldots \ldots$ \\
\hline 31 & 34.6 & 35.6 & 39.1 & 38.3 & 37.4 & 35.0 & 35.6 & 37.8 & $\ldots \ldots \ldots \ldots$ \\
\hline Mean & 32.4 & 30.7 & 33.2 & 33.1 & 32.4 & 30.6 & 31.1 & 31.0 & 26.4 \\
\hline
\end{tabular}

Urea Nitrogen.- Two of the females and four of the males were used for urea nitrogen determinations on whole blood. For the females, the pre-bleeding normal level was found to be $12.7 \mathrm{mg}$. per $100 \mathrm{cc}$., for the males, $11.0 \mathrm{mg}$. (table 54). Following hemorrhage, the level was distinctly above normal for both sexes, reaching a mean of $17.4 \mathrm{mg}$. for the females, $17.7 \mathrm{mg}$. for the males, at the end of the first post-bleeding week. Both females showed high post-bleeding urea nitrogen values, whereas the high post-bleeding mean for the males was the result of marked increases in three of the dogs, with comparatively little change in the fourth animal. In four of the five animals showing a post-bleeding rise in urea nitrogen, there was a decline toward the normal level throughout the post-bleeding period. The fifth animal, however, continued to show very high urea nitrogen levels. By the end of the twelfth post-bleeding week the urea nitrogen levels of the three animals that were observed for this length of time (one female and two males) had returned to normal.

Amino Acid Nitrogen and Creatinine.-Data on amino acid nitrogen (table 55) and creatinine (table 56), in milligrams per $100 \mathrm{cc}$. of blood, were obtained on one female and two males. Although the post-bleeding level of amino acid nitrogen appeared to be distinctly higher than 
Table 54. Values for Urea Nitrogen in Blood, in Milligrams per 100 Cubic Centimeters (Long-term study)

\begin{tabular}{|c|c|c|c|c|c|c|c|c|c|}
\hline \multirow{2}{*}{$\begin{array}{l}\text { Dog } \\
\text { No. }\end{array}$} & \multirow{2}{*}{$\begin{array}{c}\text { Pre- } \\
\text { bleeding } \\
\text { normal }\end{array}$} & \multicolumn{8}{|c|}{ Post-bleeding period } \\
\hline & & 1-2 days & l wk. & 2 wks. & 3 wks. & 4 wks. & 5 wks. & 6 wks. & 12 wks. \\
\hline \multicolumn{10}{|c|}{ Females } \\
\hline 19 & 18.02 & 18.61 & 25.07 & 20.39 & 14.83 & 18.80 & 16.94 & 18.80 & 13.21 \\
\hline 32 & 7.40 & 17.89 & 9.75 & 19.61 & 22.46 & 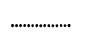 & 16.86 & 11.27 & .................. \\
\hline Mean & 12.71 & 18.25 & 17.41 & 20.00 & 18.64 & 18.80 & 16.90 & 15.03 & 13.21 \\
\hline \multicolumn{10}{|l|}{ Males } \\
\hline 17 & 13.74 & 14.23 & 11.00 & 13.71 & 14.72 & 9.11 & 11.52 & 10.86 & 11.47 \\
\hline 18 & 13.79 & 15.36 & 17.17 & 17.35 & 14.83 & 12.82 & 14.21 & 12.77 & 6.89 \\
\hline 30 & 8.88 & 6.82 & 17.78 & 25.53 & 21.33 & 21.55 & 21.88 & $\ldots \ldots \ldots \ldots \ldots$ & 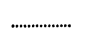 \\
\hline 31 & 7.68 & 20.51 & 25.02 & 16.55 & 30.65 & 23.99 & 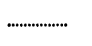 & 31.91 & 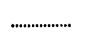 \\
\hline Mean & 11.02 & 14.23 & 17.74 & 18.28 & 20.38 & 16.87 & 15.87 & 18.51 & 9.18 \\
\hline
\end{tabular}

Table 55. Values for Amino Acid Nitrogen in Blood, in Milligrams per 100 Cubic Centimeters

(Long-term study)

\begin{tabular}{|c|c|c|c|c|c|c|c|c|c|}
\hline \multirow{2}{*}{$\begin{array}{l}\text { Dog } \\
\text { No. }\end{array}$} & \multirow{2}{*}{$\begin{array}{c}\text { Pre- } \\
\text { bleeding } \\
\text { normal }\end{array}$} & \multicolumn{8}{|c|}{ Post-bleeding period } \\
\hline & & 1-2 days & $1 \mathrm{wk}$. & 2 wks. & 3 wks. & 4 wks. & 5 wks. & 6 wks. & 12 wks. \\
\hline \multicolumn{10}{|c|}{ Female } \\
\hline 19 & 4.05 & 16.73 & 17.27 & 18.10 & 21.12 & 16.25 & 17.11 & 7.08 & 16.65 \\
\hline \multicolumn{10}{|l|}{ Males } \\
\hline 17 & 4.84 & 7.34 & 14.62 & 15.92 & 12.27 & 7.52 & 9.73 & 12.97 & 8.19 \\
\hline 18 & 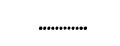 & $\ldots$ & 3.61 & 8.74 & 10.56 & 12.86 & 13.11 & 20.98 & 10.42 \\
\hline Mean & 4.84 & 7.34 & 9.11 & 12.33 & 11.41 & 10.19 & 11.42 & 16.97 & 9.30 \\
\hline
\end{tabular}

Table 56. Values for Creatinine in Blood, in Milligrams per 100 Cubic Centimeters (Long-term study)

\begin{tabular}{|c|c|c|c|c|c|c|c|c|c|}
\hline \multirow{2}{*}{$\begin{array}{l}\text { Dog } \\
\text { No. }\end{array}$} & \multirow{2}{*}{$\begin{array}{c}\text { Pre- } \\
\text { bleeding } \\
\text { normal }\end{array}$} & \multicolumn{8}{|c|}{ Post-bleeding period } \\
\hline & & 1-2 days & 1 wk. & 2 wks. & 3 wks. & 4 wks. & 5 wks. & 6 wks. & 12 wks. \\
\hline \multicolumn{10}{|c|}{ Female } \\
\hline 19 & 1.72 & 1.65 & 1.63 & 1.66 & 1.58 & 1.55 & 1.56 & 1.38 & 1.52 \\
\hline \multicolumn{10}{|l|}{ Males } \\
\hline 17 & 1.44 & 1.57 & 1.61 & 1.58 & 1.58 & 1.55 & 1.58 & 1.56 & 1.52 \\
\hline 18 & 1.81 & 1.65 & 1.74 & 1.63 & 1.70 & 1.70 & 1.66 & 1.70 & 1.70 \\
\hline Mean & 1.62 & 1.61 & 1.67 & 1.60 & 1.64 & 1.62 & 1.62 & 1.63 & 1.61 \\
\hline
\end{tabular}


the normal level, such sharp fluctuations occurred at times during the post-bleeding period that because of the limited number of normal values secured it cannot be said positively that such a condition prevailed.

The level for creatinine remained surprisingly constant in the two male animals throughout the post-bleeding period, whereas a slight decrease was noted for the female.

\section{DISCUSSION}

\section{Total Blood Volume}

In the present research, it was evident that following hemorrhage the animals could adapt themselves to a total blood volume which was appreciably less than normal. Although some restoration of total blood volume occurred, owing to the increase in plasma, complete restoration was limited by recovery in cell volume. In the short-term study, the total blood volume 30 minutes after the start of bleeding was found to be 17 per cent below the pre-bleeding mean, followed 24 hours later by recovery to a level which was only 10 per cent below, and on the third day by a value which was only 13 per cent below the pre-bleeding mean. In the long-term study, the female animals displayed total blood volumes 13 per cent below normal even six weeks after bleeding. The total blood volume of the males, however, remained below normal only two weeks after bleeding. Thereafter their total blood volume remained normal, owing to their more satisfactory recovery in cell volume.

The observations in the present study confirmed the results reported by Robertson and Bock (1919) from their work with wounded soldiers, in whom they observed marked reductions in total blood volume following hemorrhage. They noted increases in blood volume coincident with the rapid increase in the percentage of reticulated cells, or with the addition of new cells to the circulation through transfusion.

\section{Constituents of Whole Blood}

Little information was available in the literature concerning variations after hemorrhage in the protein nitrogen of the blood. Hawk and Gies (1904), working with dogs, and Buell (1919), experimenting on pigs, reported a decrease following bleeding in both the total and protein nitrogen.

In the long-term study, recovery in protein nitrogen of the whole blood was retarded by the slow recovery in the volume of red blood cells. As pointed out in the section on plasma constituents, recovery in plasma protein nitrogen, particularly that of the albumin and fibrinogen, was prompt and complete within a few days after hemorrhage.

Increases in the nomprotein nitrogen level of the blood following 
hemorrhage have been reported by a number of workers, which results are in accord with the findings in the present investigation, particularly as concerns the females, who showed increases following bleeding, with gradual return to normal during the post-bleeding period.

Increases in blood urea nitrogen would appear to account for a large portion of the increases noted in nonprotein nitrogen following hemorrhage, as shown by the work of Taylor and Lewis (1915), who as the result of observations on one dog reported a marked increase in urea nitrogen following repeated hemorrhage. Buell (1919) noted slight increases after bleeding in urea nitrogen of the blood of pigs, the increases occurring at intervals which varied in duration. An increase in blood urea was also reported by Dubois and Polonovski (1924) as existing after bleeding in both rabbits and dogs. On the other hand, BrocqRousseu, Roussel, and Gallot (1928), analyzing the blood of 36 horses at the beginning and at the end of a massive hemorrhage, found urea nitrogen of the blood to be practically unchanged. Dumas and Pagès (1930) obtained similar results on their three subjects.

In human subjects, Borst (1936) and Wood (1936) found a rather definite rise in blood urea following severe gastric hemorrhage. The former attributed the condition to an increased urea formation owing to decomposition and resorption of exuded blood, while the latter believed it might be due to a decreased kidney function as a result of oxygen-want following great loss of hemoglobin. He found that when the hemoglobin level was brought up rather rapidly to a fairly normal level by use of a continuous massive transfusion of citrated blood, the blood urea level promptly fell to within normal limits.

In the present investigation, increases in urea nitrogen of the blood were noted following hemorrhage in five of the six animals on which the determination was made. No relationship was apparent between the oxygen-combining capacity of the animals and the level of urea nitrogen. Therefore, observations on these animals were not in accord with Wood's suggestion that increases in urea nitrogen follow kidney failure due to lowered hemoglobin content of the blood.

A number of research workers have reported on the effects of hemorrhage upon the amino acid content of the blood. György and Zunz (1915) stated that although the amino acid content of the blood of dogs remained remarkably constant under normal conditions copious bleeding resulted in a slight increase, although when Ringer's solution was injected simultaneously the amino acid content tended to decrease. This latter observation is probably due to the effect of dilution of the blood with Ringer's solution. Taylor and Lewis (1915a), in a similar experiment, observed a rise in the amino acid nitrogen of the blood of one dog, which they attributed to the release of stored amino acids, or amino acids derived from the hydrolysis of tissue or serum proteins. Tochowicz (1936-37) observed normal levels of amino acids in both the whole blood and plasma in patients with secondary anemia. 
In the current study, there seemed to be a rise in the amino acid nitrogen of the blood following bleeding. The limited number of observations made, however, (on three dogs) does not warrant too definite conclusions on this point.

No data were found in the literature covering the effect of hemorrhage on the creatinine level. In this research, there appeared to be a decrease in the creatinine content of the blood of the one female on which the determination was made, whereas in the two male animals no alteration was noted.

\section{SUMMARY \\ Total Blood Volume •}

In the short-term study, a 17 per cent decrease in blood volume was observed 30 minutes after the start of bleeding, followed 24 hours later by recovery to a level which was only 10 per cent below the pre-bleeding mean, with slightly lower values throughout the balance of the postbleeding period. In the long-term study, the females showed a 13 per cent decrease as a result of hemorrhage at the end of the first postbleeding week, and no recovery during the balance of the post-bleeding period. The males showed a 7 per cent decrease at the end of the first post-bleeding week, followed by recovery to the pre-bleeding level early in the post-bleeding period.

\section{Constituents of Whole Blood}

In the long-term study, for the females the protein nitrogen remained distinctly below the pre-bleeding mean during the post-bleeding period and reflected their failure to restore cell volume. Similarly for the males, although some recovery was noted, the protein nitrogen level remained distinctly below the pre-bleeding mean throughout the post-bleeding period. For both males and females the mean nonprotein nitrogen level fluctuated throughout the post-bleeding period, the females showing slightly higher means.

In the urea nitrogen levels, fluctuations were observed for both males and females during the post-bleeding period, the values in general being above the pre-bleeding means.

There were sharp fluctuations in the amino acid nitrogen level throughout the post-bleeding period, with means higher than pre-bleeding values.

The mean pre-bleeding creatinine level was maintained throughout the post-bleeding period by the two males, whereas the one female showed a slight decrease. 


\section{GENERAL DISCUSSION}

$\mathrm{H}^{\mathrm{s}}$ EMORRIAGIC and hypochromic anemias occur often enough and are of sufficient human significance to be included in the classifications of anemias proposed by authorities and presented by Downey (1938) in a recent comprehensive resumé of the field. Wintrobe and Beebe (1933) listed 15 different names that had been used and that were apparently considered as synonymous terms applicable to the condition described by them as idiopathic hypochromic anemia. The use of the term "idiopathic" indicates uncertainty as to the causes of this condition. "Hypochromic" is associated with a deficiency of the pigment, hemoglobin; "microcytic," a term proposed by other workers (Witts, 1931 ; Vanderhoof and Davis, 1932; and Wintrobe, 1934), is applied to undersized erythrocytes.

Scattered statements in the literature support the theory that hemorrhages in different parts of the body may cause hypochromic anemia. It occurs much more frequently in women, especially between the ages of 20 and 50 years, than in men (Witts, 1931). Fullerton (1936) claimed that the loss of iron during the reproductive period of women was of great importance and that cumulative losses due to menstruation, pregnancy, and lactation resulted in a gradually falling hemoglobin level until the menopause, after which a marked rise was noted. Strauss and Castle (1933) regarded the development of blood in the fetus as equivalent to a blood loss in the mother and stated that repeated pregnancies have resulted in marked hypochromia. Others cited periodic menstrual losses as possible causes (Witts, 1931; Wintrobe and Beebe, 1933; Wright, 1933 ; Barer, Fowler, and Baldridge, 1934-35 ; and Fowler and Barer, 1937). On the other hand, Haden and Singleton (1933) did not regard excessive menstrual hemorrliages alone as responsible for anemia since in their series of cases menstrual disorders were usually relieved when the blood picture returned to normal. Vanderhoof and Davis (1932) did not consider periodic blood losses as important for the five women whom they studied.

The similarity between the blood picture observed following acute or recurrent blood losses and that noted in hypochromic anemia has been emphasized (Heath, 1933; Castle and Minot, 1936; and Fowler and Barer, 1937). Witts (1931) stated that the occurrence of this type of anemia in men was indicative of blood loss, the source of which should be located.

The blood pictures cited for hypochromic or microcytic anemias are paralleled by those reported for hemorrhagic anemia as based on the results of this investigation. Hemoglobin, whether measured in terms 
of the level, or of the color index, or of mean corpuscular hemoglobin concentration, has always been found to be low in hypochromic anemia (Wintrobe and Beebe, 1933; Haden and Singleton, 1933; Wintrobe, 1934; Sturgis, 1938; McLean, 1938; Fowler and Barer, 1939). In the experimental animals used in the current studies hemoglobin failed to return to normal at any time during the course of the research. Red cell counts have been reported as reduced in number (Wintrobe, 1934; Castle and Minot, 1936), normal or relatively high (Wintrobe and Beebe, 1933; Haden and Singleton, 1933), or increased when paralleled by microcytosis (Sturgis, 1938). In the experimental animals red cell counts were low in the early stages of recovery, but increased rapidly in number, reaching ultimately a level considerably above normal. Most investigators who have studied cell size have reported alterations ranging from slight to marked decreases (Wintrobe, 1929-30; Price-Jones, 1932; Wintrobe and Beebe, 1933; Wintrobe, 1934; Castle and Minot, 1936; Sturgis, 1938; Bethell, Isaacs, Goldhamer, and Sturgis, 1938; and Fowler and Barer, 1939). In the present research, decreases in cell size were noted invariably, the extent of decrease varying with the blood losses to. which the animals were subjected.

Thus similarities noted in the blood pictures of the experimental animals in which hemorrhagic anemia had been induced, and of human subjects afflicted with idiopathic hypochromic anemia, substantiate the hypothesis that the latter type is frequently the result of hemorrhage. These studies do not, of course, preclude the existence of other causes of hypochromic anemia.

To secure a comprehensive picture of blood changes occurring in hemorrhagic anemia, the dogs used in these experiments were maintained under controlled conditions and subjected to bleedings of such magnitude as to simulate hemorrhages occurring in human subjects such as those involved in accidents, operations, childbirth, menorrhagia, and the like.

It must be appreciated that these same controlled conditions made it possible to secure a more accurate picture of alterations in the blood caused by hemorrhage than would have been possible with human subjects, since the animals selected were normal healthy dogs, maintained on a balanced diet and subjected to bleedings regulated in size and frequency, under circumstances that were not complicated by other pathological or physiological influences.

Observation showed that at progressive stages in recovery certain combinations of blood measures were more satisfactory criteria than others, the combination varying from time to time. Therefore, it is essential that the changing significance of these measures be kept in mind in assessing the status of individuals who have experienced blood losses and in following the progress of recovery in anemic persons.

Hemorrhage results in the loss of both plasma and cells. In this investigation, immediately after acute bleeding, the hematocrit, the red cell count, and the levels of constituents such as hemoglobin showed 
little change and gave no indication of the extent of the hemorrhage. Shortly thereafter dilution with interstitial fluid occurred bringing about a rapid restoration of plasma volume, with the result that hematocrits, red cell counts, and hemoglobin decreased simultaneously. The replacement of erythrocytes was a slow process which in these experiments was not complete even after many weeks. Marked shifts in these measurements are therefore characteristic of the adjustments taking place soon after a severe hemorrhage and are consequently significant in establishing or verifying the occurrence, the recency, or the extent of hemorrhage.

Certain measures are of infinitely greater significance than others as a means of establishing the status of a person following blood loss. Thus judgment of an individual's condition, based on the hemoglobin level, red cell count, or red cell hematocrit, in the very early stages of recovery would give an erroneous conception and create a false sense of security. It is therefore evident that to secure an adequate basis for assessment it is necessary to secure total plasma volume and total cell volume data to be used in conjunction with other measures. In the later stages of recovery also, red cell counts are of limited usefulness, since an increase in count may be associated with a decrease in cell size, resulting in a total cell volume still markedly below the normal level. For example, a high count, associated with small size, suggests repeated or long-continued blood losses; a low red cell count, with cells of normal size, is indicative of a recent severe hemorrhage. It may be concluded, therefore, that several carefully selected combinations of blood measurements will more adequately evaluate the condition of a person after bleeding has taken place than any single criterion.

The current research, which covered a considerable period of time, demonstrated that certain factors returned to normal rapidly, whereas others (hemoglobin, red cell volume, and red cell size) returned to normal slowly, in some instances not at all, and therefore needed special consideration. The latter correspond to those factors which have been shown clinically to be subnormal in human subjects suffering from the types of anemia under discussion. This investigation has established the relative merits of different measures of recovery.

The synthetic diet used in the present experiments permitted limited recovery in cell volume and hemoglobin. It was evident that the diet, although quite adequate for maintaining animals in a normal condition over long periods of time, was of limited usefulness in restoring the blood following bleeding. Although the iron content was fairly generous, it is possible that a higher iron intake would have resulted in more rapid regeneration. Other undetermined factors may have played a role also in limiting recovery.

Hypochromic anemia caused by blood losses constitutes a real nutritional problem. It is obvious that the characteristics of hemorrlagic anemia observed in these studies closely resemble those which have been 
reported by other observers as characteristic of hypochromic microcytic anemia in humans. The advantages of conducting studies like those reported in this bulletin are self-evident. The normal blood picture of dogs has been portrayed, thus affording a basis for selecting suitable animals for the experiments and simultaneously establishing goals of achievement for corrective nutritional programs. Procedures employing hemorrhages have been evolved and described, by the use of which hypochromic microcytic anemias of varying degrees of severity may be produced. Blood pictures characteristic of different phases of the posthemorrhagic period have been set forth and the factors which return to normal with difficulty have been located.

The techniques described, the normals established, and the location of the factors which improve slowly, make it possible to produce in animals an hypochromic microcytic anemia associated with blood losses, and to evaluate dietary regimes designed to restore the blood picture to normal.

\section{SUMMARY}

1. This bulletin presents a comprehensive description of changes occurring in the blood pictures of 19 male and female dogs maintained on what is believed to be an adequate synthetic diet for adult dogs and subjected to massive bleedings, after which they were observed for periods varying in length.

2. Detailed summaries of the findings are presented at the end of each section-normal blood constituents, page 32; erythrocytes and leucocytes, pages 60 to 63 ; blood plasma, pages 78 to 79 ; and whole blood, page 87 .

3. Within 30 minutes after hemorrhage, the most outstanding blood changes noted are the decrease in total blood volume and the drop in cell volume per cent, paralleling an immediate increase in plasma volume and slightly enlarged erythrocytes. At this time, dilution with interstitial fluid is not sufficient to modify very much the levels of the several blood constituents.

4. By the end of the first week, a typical blood picture of hemorrhagic anemia of short duration is obtained. The erythrocyte volume is distinctly below normal, the plasma volume exceeds somewhat the prebleeding level, while the total blood volume is still below normal. Serum, albumin, and fibrinogen levels are restored to normal, whereas total nitrogen in the blood, hemoglobin, serum globulin, and nonprotein nitrogen are below the pre-bleeding means. Hypochromia is observed. The levels of the plasma calcium and phosphorus fractions are below 
normal, but the totals for both exceed the amounts originally present in the plasma. No significant leucocytosis is noted. Red cell counts are below normal and the size of the cells is decreased.

5. In the animals observed 6 to 12 weeks, a blood picture typical of hemorrhagic anemia of long standing is found. Both total blood volume and plasma volume approach pre-bleeding levels, but the total erythrocyte volume is still below normal. Relatively little recovery is noted in hemoglobin and total protein in the blood. The erythrocyte counts may be normal or above normal, but the individual cell volume or diameter is decreased, a relationship reflected in the significant negative correlation between these variables. Thus both hypochromia and microcytosis are present. In many cases, the progress observed in the males is greater than that noted in the females.

6. No single measurement adequately assesses the status of an individual after the loss of significant amounts of blood. The most serious blood changes involve the modified erythrocyte picture-total cell volume, cell volume per cent, erythrocyte count in relation to individual cell volume and diameter, and hemoglobin content of the cells.

7. The rate of erythrocyte regeneration depends upon the degree of physiological stress caused by hemorrhage, upon the reserves of cellbuilding materials in the animal body, and to a limited extent, upon the amount of food consumed.

8. Statistically significant relationships were observed between some of the blood constituents before bleeding, in others after bleeding.

9. Animals given a diet that will maintain a normal blood picture under ordinary conditions develop an anemia characterized by hypochromia and microcytosis when subjected to severe or recurrent hemorrhages. 


\section{LITERATURE CITED}

Adolph, E. F., Gerbasi, M. J., and Lepore, M. J. 1933. The rate of entrance of fluid into the blood in hemorrhage. Am. J. Physiol. 104:502-517.

Arnold, H. R., Carrier, E. B., Smith, H. P., and Whipple, G. H. 1921. Blood volume studies. $\mathrm{V}$. The carbon monoxide method-its accuracy and limitations. Am. J. Physiol. 56:313-327.

Arnold, R. M., and Mendel, L. B. 1927. Interrelationships between the chemical composition of the blood and the lymph of the dog. J. Biol. Chem. 72: 189-211.

Ashley, A., and Guest, G. M. 1934. The distribution of blood phosphorus after suppression of renal function. J. Clin. Investigation 13:219-235.

Atrinson, H. V., and Ers, H. N. 1922. Chemical changes of the blood under the influence of drugs. J. Biol. Chem. 52:5-10.

Austin, J. H., Stillman, E., and Van Slyke, D. D. 1921. Factors governing the excretion rate of urea. J. Biol. Chem. 46:91-112.

BANG, Ivar. 1915-16. Untersuchungen über Reststickstoff des Blutes. I. Mitteilung. Biochem. Z. 72:104-118.

Barer, A. P., and Fowler, W. M. 1936. The blood loss during normal menstruation. Am. J. Obstet. Gynecol. 31:979-986.

Barer, A. P., Fowler, W. M., and Baldridge, C. W. 1934-35. Blood loss during normal menstruation. Proc. Soc. Exptl. Biol. Med. 32:1458-1459.

Bentrie, F. 1928. A micro-method for the colorimetric determination of urea in blood. Biochem. J. 22:711-712.

Bellis, C. J., and ScotT, F. H. 1935. The alteration of protein distribution, in vitro, between corpuscles and plasma, caused by isosmotic and hyperosmotic solutions. J. Biol. Chem. 111:17-42.

Bennett, Mary A. 1926. Some changes in the acid-base equilibrium of the blood caused by hemorrhage. J. Biol. Chem. 69:675-692.

Bethell, F. H., IsaAcs, R., Goldhamer, S. M., and Sturgis, C. C. 1938. BloodA review of the recent literature. Arch. Internal Med. 61:923-1011.

Biedl, A., and von Decastello, A. 1901. Ueber Aenderungen des Blutbildes nach Unterbrechung des Lymphzuflusses. Arch. ges. Physiol. (Pflügers) 86: 259-290.

Bloor, W. R. 1918. Methods for the determination of phosphoric acid in small amounts of blood. J. Biol. Chem. 36:33-48.

Bock, J. C. 1917. The amino-acid nitrogen content of the blood of various species. J. Biol. Chem. 29:191-198.

Bodansky, M., and Dressler, O. G. 1927. The distribution of water and cholesterol in the blood in experimental anemia. Quart. J. Exptl. Physiol. 17:157-160.

Borst, J. G. G. 1936. Keukenzoutretentie en Azotaemie na Groote Maagbloedingen I. [Sodium chloride retention and uremia after extensive stomach bleeding, I.] Nederlandsch Tijdschr. Geneeskunde 80:2310-2319. Cited by Chem. Abstracts 30:5642.

Breuer, R., and von Seiller, R. F. 1903. Ueber den Einfluss der Kastration auf den Blutbefund weiblicher Tiere. Arch. exptl. Path. Pharmakol. 50:169-198.

Briggs, A. P. 1924. Some applications of the colorimetric phosphate method. J. Biol. Chem. 59:255-264.

Briggs, A. P., Koecing, Irene, Doisy, E. A., and Weber, C. J. 1923-24. Some changes in the composition of blood due to the injection of insulin. J. Biol. Chem. 58:721-730. 
Broce-Rousseu, Roussel, G., and Gallot. 1928. Dosage de l'urée dans les prises différentes d'une même saignée. Comp. rend. soc. biol. 99:1203-1204.

Brodin, P., Richet, C., and Saint-Grrons, Fr. 1919-20. De la quantité de sang (masse de sang) mesurée par le nombre des hématies. J. physiol. path. gén. 18:8-26.

Brodin, P., Saint-Girons, Fr., and Richet, C. 1918. Densité, hématies, leucocytes, et quantité de sang chez 47 chiens a l'etat normal. Compt. rend. soc. biol. $81: 681-684$.

Brull, L. 1930. Contribution a l'étude de l'état physico-chemique des constituants minéraux et du glucose plasmatiques. Arch. intern. physiol. 32:138-236.

Bruner, H. D., and Wakerlin, G. E. 1937. The blood picture of the normal dog. Proc. Soc. Exptl. Biol. Med. 36:667-670.

Buddington, W. T., and TAylor, G. W. 1938. The loss of blood in certain standard operations for malignant disease. New Engl. J. Med. 218:285-287.

Buell, Mary V. 1919. Studies of blood regeneration. II. Effect of hemorrhage on nitrogen metabolism. J. Biol. Chem. 40:63-77. $56: 97-107$.

Burket, W. C. 1917. Changes in the peripheral blood consequent upon the diversion of the splenic blood into the general circulation. J. Exptl. Med. 26:849-865.

Burnetr, S. H. 1917. Clinical pathology of the blood of domesticated animals. The Macmillan Co., New York.

Burnett, S. H., and Traum, J. 1905. The clinical examination of the blood of the dog. Proc. Am. Vet. Med. Assoc., 42nd Meeting, pp. 349-362.

Busch, F. C., and Van Bergen, C. 1902. Dog's blood. Differential counts of leucocytes. J. Med. Research 8:408-414.

Cahn, T., and Houget, J. 1933. La composition moyenne du muscle, du foie et du sang du chien. Compt. rend. soc. biol. 112:1319-1321.

Camero, A. R., and Krumbinar, E. B. 1933. The effect of experimental massive hemorrhages on the size of the red blood cell in dogs. Am. J. Physiol. 103:407-416.

Castle, W. B., and Minot, G. R. 1936. Pathological physiology and clinical description of the anemias. Oxford University Press, New York.

Charles, E. 1931-32. Comparative studies of sex differences in calcium and magnesium content of serum. Quart. J. Exptl. Physiol. 21:81-91.

Coller, F. A., and Maddock, W. G. 1932. Dehydration attendant on surgical operations. J. Am. Med. Assoc. 99:875-880.

Collip, J. B., Clark, E. P., and Scott, J. W. 1925. The effect of a parathyroid hormone on normal animals. J. Biol. Chem. 63:439-460.

Costantino, A. 1913. II. Der durch Formol titrierbare Aminosäuerstickstoff in den Blutkörperchen und im Serum des Blutes von hungernden und ernährten Tieren. Biochem. Z. 55:402-410.

Courmont, J., and Lesieur, Ch. 1901. La polynucléose de la rage. J. physiol. path. gén. 3:599-610.

Culitane, K. 1930. Does cabbage fed to rabbits increase serum calcium? A reply to Kapsinow and Underhill. J. Biol. Chem. 86:113-116.

Cuvelier, R., and Patorr, G. 1932. La régénération des albumines du sérum sanguin après la saignée chez le chien. Compt. rend. soc. biol. 111:16-18.

Darrow, D. C., and CAry, M. K. 1934. The effect of nutritional hypoproteinemia on the electrolyte pattern and calcium concentration of serum. J. Biol. Chem. 105:327-334. 
DAwson, P. M. 1900-01. Effects of venous haemorrhage and intravenous infusion in dogs. Am. J. Physiol. 4:1-24.

Downey, Hal. 1938. Handbook of hematology. Vol. 3. Paul B. Hoeber, New York.

Draper, J. W. 1917. Intestinal obstruction, complete and incomplete. J. Am. Med. Assoc. 69:1768-1771.

Dubois, C., and.Polonovski, M. 1924. Influence de la saignée sur la concentration de l'urée sanguine. Compt. rend. soc. biol. 90:217-218.

Dumas, A., and Pagìs, G. 1930. Sur les variations de l'urée et des chlorures sanguins au cours de la saignée. Comp. rend. soc. biol. 103:1030-1031.

Ellenderger, W. 1887. Handbuch der Histologie und Physiologie der Haussäugethiere. Histologie, pp. 127-142.

Emmons, W. F. 1927-28. Interrelation of number, volume, diameter, and area of mammalian erythrocytes. J. Physiol. 64:215-228.

FALiK, E., and Bielinski, Z. 1931. Recherches sur le diamètre des globules rouges. Variations pendant l'inanition. Compt. rend. soc. biol. 108:161-164.

Fishiberg, E. H. 1929. The relations of the serum proteins and lipids to the osmotic pressure. J. Biol. Chem. 81:205-214.

Fisher, N. F., and Larson, E. 1925. Effects produced by extracts of parathyroid glands on normal and parathyroidectomized dogs. Am. J. Physiol. 75: 93-106.

Fisher, R. A. 1928. Statistical methods for research workers. Oliver and Boyd, London.

Folin, O. 1922. A system of blood analysis. IV. A revision of the method for determining uric acid. J. Biol. Chem. 54:153-170.

Folin, O., and Denis, W. 1916. Nitrogen determinations by direct nesslerization. I. Total nitrogen in urine. II. Non-protein- nitrogen in blood. J. Biol. Chem. 26:473-489; 491-496.

Folin, O., and Svedberg, A. 1930. Micro methods for the determination of nonprotein nitrogen, urea, uric acid, and sugar in unlaked blood. J. Biol. Chem. 88:85-96.

Folin, O., and WU, H. 1919. A system of blood analysis. J. Biol. Chem. 38: $81-110$.

Formad, H. F. 1888. Comparative studies of mammalian blood; with special reference to the microscopical diagnosis of blood stains in criminal cases. J. Comp. Med. Surg., New. York and Philadelphia, 9:254-312.

Foster, D, P, and Whipple, G. H. 1921-22. Blood fibrin studies. II. Normal fibrin values and the influence of diet. Am. J. Physiol. 58:379-392.

Fowler, W. M., and BArer, A. P. 1937. The etiology and treatment of idiopathic hypochromic anemia. Am. J. Med. Sci. 194:625-635.

. 1939. The treatment of iron deficiency anemias. J. Am. Med. Assoc. 112:110-112.

Franke, E., and Benedict, S. R. 1920-21. A method for the determination of blood volume. J. Lab. Clin. Med. 6:618-624.

Fritscri, G. 1920. Das Blut der Haustiere mit neueren Methoden untersucht. II. Untersuchung des Kaninchen-, Hühner-, und Taubenblutes. Arch. ges. Physiol. (Pflügers) 181:78-105.

Fullerton, H. W. 1936. Anemia in poor class women. Brit. Med. J. 2:523-528.

Furno, A. 1913. Ricerche sperimentali ematologiche ed anatomo-patologiche intorno all 'emolisi da siero negli animali normali e splenectomizzati. Sperimentale $67: 639-752$.

Gasser, H. S., Erlanger, J., and Meek, W. J. 1919. Studies in secondary traumatic shock. IV. The blood volume changes and the effect of gum acacia on their development. Am. J. Physiol. 50:31-53. 
Gatcir, W. D., and Litrle, W. D. 1924. Amount of blood lost during some of the more common operations. J. Am. Med. Assoc. 83:1075-1076.

Gerschman, Rebecca. 1931. Calcium et phosphore du plasma sanguin des chiens hypophysoprives. Compt. rend. soc. biol. 108:494-495.

Gibson, J. G., 2nd, Keeley, J. L., and Pijonn, M. 1938. The blood volume of normal dogs. Am. J. Physiol. 121:800-806.

Goodsll, A. 1910. The numbers, proportions, and characters of the red and white blood corpuscles in certain animals. J. Path. Bact. 14:195-199.

Goodall, A, Gulland, G. L., and Paton, D. N. 1903-04. Digestion leucocytosis in normal and in spleenless dogs. J. Physiol. 30:1-9.

Goodpasture, E. W. 1914. Fibrinogen. II. The association of liver and intestine in rapid regeneration of fibrinogen. Am. J. Physiol. 33:70-85.

GovaERTs, P. 1925. Influence du rapport albumines-globulines sur la "pression osmotique des protéines du sérum. Compt. rend. soc. biol. 93:441-443.

1926. Quotient albumines-globulines et pression osmotique des protéines du sérum. Compt. rend. soc. biol. 95:724-726.

Gran, H. C. 1924. Cell volume and electrical conductivity of blood. J. Biol. Chem. 59:33-40.

Grénant, N., and QuinQUad, E. 1882. Mémoires présentés. Physiologic-mesure du volume de sang contenu dans l'organisme d'un Mammifère vivant, mémoire de Mm. Gréhant et E. Quinquad, présenté par M. Marcy. (Extrait par les auteurs.) (Renvoi au concours de médicine et chirurgie.) Compt. rend. acad. sci. 94:1450-1453.

Green wald, I. 1915. The estimation of non-protein nitrogen in blood. J. Biol. Chem. 21:61-68.

1918. The estimation of non-protein nitrogen in blood. J. Biol. Chem. 34:97-101.

1931. The relation of the concentration of calcium to that of protein and inorganic phosphate in the serum. J. Biol. Chem. 93:551-561.

Gyoergy, P., and Zunz, E. 1915. A contribution to the study of the amino-acid content of the blood. J. Biol. Chem. 21:511-537.

HADEN, R. L. 1924-25. The volume and hemoglobin content of the erythrocytes in health and disease. Folia Haematol. 31:113-135.

1930. The technique of determination of the relative mass, the individual cell volume, and the volume index of the erythrocytes of man. J. Lab. Clin. Med. 15:736-746.

1932. Clinical significance of volume and hemoglobin content of the red blood cell. Arch. Internal Med. 49:1032-1057.

Haden, R L., and OrR, T. G. 1923. Chemical changes in the blood of the dog after intestinal obstruction. J. Exptl. Med. 37:365-375.

1923. Chemical changes in the blood of the dog after obstruction of esophagus and of the cardiac end of the stomach. J. Exptl. Med. 38: 477-485.

1925. Chemical findings in the blood of the normal dog. J. Biol. Chem. 65:479-481.

Haden, R. L., and Singleton, R. M. 1933. Disturbances of menstruation due to simple achlorhydric anemia. Am. J. Obstet. Gynecol. 26:330-335.

HArris, D. T. 1920. The value of the vital-red method as a clinical means for the estimation of the volume of the blood. Brit. J. Exptl. Path. 1:142-158.

HAwk, P. B., and Gies, W. J. 1904. The influence of external hemorrhage on chemical changes in the organism, with particular reference to proteid catabolism. Am. J. Physiol. 11:171-236. 
Hayem, G. 1889. Du sang et de ses altérations anatomiques. G. Masson, Paris. Heati, C. W. 1933. The volume and hemoglobin content of the red blood corpuscles in the light of recent knowledge of anemia. New Engl. J. Med. 209: 173-180.

Holman, R. L., Mahoney, E. B., and Whipple, G. H. 1934. Blood plasma protein regeneration controlled by diet. I. Liver and casein as potent diet factors. J. Exptl. Med. 59:251-267.

Holt, G. W. 1931-32. Heparin in blood calcium analyses. Proc. Soc. Exptl. Biol. Med. 29:315-316.

Hooper, C. W., Robscheit, F. S., and Whipple, G. H. 1920. Blood regeneration following simple anemia. III. Influence of bread and milk, crackermeal, rice, and potato, casein, and gliadin in varying amounts and combinations. V. Influence of Blaud's pills and hemoglobin. Am. J. Physiol. 53:205-235; 263-282.

Hooper, C. W., Smitit, H. P., Belt, A. E., and Whipple, G. H. 1920. Blood volume studies. I. Experimental control of a dye blood volume method. Am. J. Physiol. 51:205-220.

Howe, P. E. 1921a. The use of sodium sulfate as the globulin precipitant in the determination of proteins in blood. J. Biol. Chem. 49:93-107.

1921b. The determination of proteins in blood- a micro-method. J. Biol. Chem. 49:109-113.

Hubbell, H. J., and Rose, M. S. 1937. The influence of sex on utilization of iron. J. Nutrition 13(Supplement):8-9.

Jolly, J. 1923. Traité technique d'hématologie; morphologie, histogenèse, histophysiologie, histopathologie. A. Maloine et fils, Paris.

Jones, H. W., Widing, H., and Nelson, L. 1931. Blood donors. A study of the effect in donors of repeated blood loss. J. Am. Med. Assoc. 96:1297-1300.

Kapsinow, R., and Underinill, F. P. 1929. Does cabbage fed to rabbits increase serum calcium? J. Biol. Chem. 82:377-384.

KARR, W. G. 1920. Some effects of water-soluble vitamine upon nutrition. J. Biol. Chem. 44:255-276.

KuUftineil, L., and Kisch, F. 1927a. Der Kalium- und Calciumgehalt des Gesamtblutes bei Blutkrankeiten. Klin. Wochschr. 6:1328-1329.

1927b. Experimentelle Studien über das Verhalten des Kalium- und Calciumspiegels im Gesamtblut anämisch gemachter Tiere. Klin. Wochenschr. 6:1797-1798.

K $\Lambda$ Y, H. D., and Вугом, F. B. 1927. Blood-phosphorus in health and disease. I. The distribution of phosphorus in human blood in health. Brit. J. Exptl. Path. 8:240-253.

KeItir, N. M. 1919. Reports of the special investigation committee on surgical shock and allied conditions. IX. Blood volume changes in wound shock and primary haemorrhage. Med. Research Council, Special Rep. Series No. 27.

Keiti, N. M., Rowntree, L. G., and Geragitit, J. T. 1915. A method for the determination of plasma and blood volume. Arch. Internal Med. 16: 547-576.

Kerr, W. J., Hurwitz, S. H., and Whipple, G. H. 1918-19. Regeneration of blood serum proteins. I. Influence of fasting upon curve of protein regeneration following plasma depletion. II. Influence of diet upon curve of protein regeneration following plasma depletion. Am. J. Physiol. 47: $356-369$; 370-378.

KING, E. J. 1932. The colorimetric determination of phosphorus. Biochem. J. 26: 292-297. 
Klieneberger, C. 1927. Die Blutmorphologie der Laboratoriumstiere; neue morphologische und anatomische Untersuchungen und Anhangsuntersuchungen normaler Physiologie. 2. Aufl. von Prof. Dr. Carl Klieneberger, Leipzig.

Koch, F. C., and McMeekin, T. L. 1924. A new direct nesslerization microKjeldahl method and a modification of the Nessler-Folin reagent for ammonia. J. Am. Chem. Soc. 46:2066-2069.

Kohanawa, C. 1928. Beiträge zur vergleischenden Morphologie des Blutes der gesunden Haussäugetiere. Folia Haematol. 36:174-247.

KuHL, P. 1919. Das Blut der Haustiere mit neueren Methoden untersucht. I. Untersuchung des Pferde-, Rinder-, und Hundeblutes. Arch. ges. Physiol. (Pflügers) 176:263-264.

LAmson, P. D. 1915. The role of the liver in acute polycythaemia: A mechanism for the regulation of the red corpuscle content of the blood. J. Pharmacol. $7: 169-224$.

LeE, F. W., and Whipple, G. H. 1921. Blood volume studies. VI. Plasma volume as determined by hemoglobin injection. Am. J. Physiol. 56:328-335.

Leichisenring, Jane M., Biester, Alice, ct al. 1932. Blood regeneration studies. II. Observations on the blood of normal dogs with special reference to the measurement of volume, erythrocytes, leucocytes, and nitrogenous constituents. Am. J. Physiol. 99:391-397.

Leichisenring, Jane M., and Hoenig, Hortense, H. 1931. Blood regeneration studies. I. Changes in the volume, number, and size of the erythrocytes in hemorrhagic anemia. Am. J. Physiol. 98:636-643.

LENZE, P. 1909. Ein Beitrag zur Form und Formveränderung der Erythrocyten und ihr Verhältnis zu den Blutplättchen bei unseren Haustieren. Arnswalde. (Quoted by Scarborough.)

Leverton, R. M., and Roberts, L. J. 1937. The iron metabolism of normal young women during consecutive menstrual cycles. J. Nutrition 13:65-95.

Lewinski, J. 1903. Beobachtungen über den Gehalt des Blutplasmas an Serum albumin, Serum globulin, und Fibrinogen. Arch. ges. Physiol. (Pflügers) 100:611-633.

LudwiG, H. 1931. Zur Funktion der "Blutdepots." (Ein Versuch zum Nachweis von "Plasmadepots.") Z. ges. expt1. Med. 80:36-52.

McKAy, C. M. 1928. The influence of protein, blood, liver, fat, iron, and potassium in the diet upon the rate of blood regeneration after hemorrhage in the rat and dog. Am. J. Physiol. 84:16-35.

McKenty, F. D. 1937. Measurement of blood loss in nose and throat operations. Can. Med. Assoc. J. 36:611-613.

McLEAN, J. A. 1938. Hematocrit determinations in normal and abnormal blood. Med. J. Australia 2:770-773.

McNaught, J. B., Scott, V. C., Woods, F. M., and Whipple, G. H. 1936. Blood plasma protein regeneration controlled by diet. Effects of plant proteins compared with animal proteins. The influence of fasting and infection. J. Exptl. Med. 63:277-301.

Madden, S. C., Winslow, P. M., Howland, J. W., and Whipple, G. H. 1937. Blood plasma protein regeneration as influenced by infection, digestive disturbances, thyroid, and food protein. A deficiency state related to protein depletion. J. Exptl. Med. 65:431-454.

Magnan, A. 1911. Recherches sur les dimensions des globules sanguins chez les oiseaux. Compt. rend. soc. biol. 71:495-496.

Malan, A. I. 1928. Studies in mineral metabolism. IX. The phosphorus partition of blood in anaemia of cattle and sheep. J. Agr. Sci. 18:401-405. 
Manassein, V. 1872. Ueber die Dimension der rothen Blutkörperchen unter verschiedenen Einflüssen. Inaug.-Diss. Tübingen. (Quoted by Scarborough.)

ManN, F. C. 1915. Shock and haemorrhage: an experimental study. Surg., Gynecol., Obstet. 21:430-439.

1916. Some bodily changes during anesthesia. An experimental study. J. Am. Med. Assoc. 67:172-175.

Marsitall, E. K., JR., and Davis, D. M. 1914. Urea: Its distribution in and elimination from the body. J. Biol. Chem. 18:53-80.

Martin, J. W., and Myers, J. T. 1934-35. The effects of blood transfusions on donors. J. Lab. Clin. Med. 20:593-597.

Matriew, C. W. 1927. The plasma proteins of normal dogs. J. Biol. Chem. 74: $557-560$.

Mayerson, H. S. 1930. The blood cytology of dogs. Anat. Record 47:239-250.

Mayerson, H. S., and Laurens, H. 1930-31. Blood studies in hemorrhagic anemia. J. Nutrition 3:453-463.

MeEK, W. J., and Gasser, H. S. 1918-19. Blood volume. A method for its determination with data for dogs, cats, and rabbits. Am. J. Physiol. 47:302-317.

Melnick, D., and Cowgill, G. R. 1937. The influence of prolonged, intensive plasmapheresis upon the ability of the organism to regenerate serum protein. J. Exptl. Med. 66:493-508.

Melnick, D., Cowgill, G. R., and Burack, Ethel. 1936. The influence of diet upon the regeneration of serum protein. II. Potency ratios of serum protein, lactalbumin, and casein, and the effect of tissue protein catabolism on the formation of serum protein. J. Exptl. Med. 64:897-920.

Mirvish, L., and Bosman, L. P. 1927-28. The influence of the internal secretions of the ovary on the calcium blood level and on calcium metabolism. Quart. J. Exptl. Physiol. 18:11-27.

Moore, N. S., and Stewart, H. J. 1930-31. The variations of the specific gravity of the plasma of the blood and the means available for altering it. J. Clin. Investigation 9:423-442.

Morawitz, P. 1906. Beobachtungen über den Wiederersatz der Bluteiweisskörper. Beitr. Chem. Physiol. Path. 7:153-164.

Morgulis, S., and Edwards, A. C. 1924. Chemical changes in the blood during fasting and subsequent refeeding. Am. J. Physiol. 68:477-498.

Murpiry, W. P. 1933. The diagnosis and treatment of secondary anemia. New Engl. J. Med. 209:331-333.

Murpiy, W. P., and Fitzhugh, G. 1930. Red blood cell size in anemia; its value in differential diagnosis. Arch. Internal Med. 46:440-457.

Musser, J. H., JR., and KrumbiaAr, E. B. 1913. The relation of the spleen to blood destruction and regeneration and to hemolytic jaundice. VI. The blood picture at various periods after splenectomy. J. Exptl. Med. 18: 487-493. 576-580.

Nassau, E. 1913-14. Das Blutbild beim Hunde mit Eckscher Fistel. Arch. exptl. Path. Pharmakol. 75:123-142.

Nelson, L. 1909. Ueber eine Methode der Bestimmung der Gesamtblutmenge beim Tier nebst Bemerkungen über die Veränderungen der letzteren bei Hunger und Mast. Arch. exptl. Path. Pharmakol. 60:340-344.

Nicol $\Lambda$ S, J., and Dumoulin, F. 1903. Influence de la splénectomie sur les leucocytes du sang chez le chien. J. physiol. path., gén. 5:1073-1080.

Nitzescu, I., and Runcesnu, I. 1927. L'influence de l'hémorrhagie sur le calcium et le phosphore minéral du sang. Compt. rend. soc. biol. 97:11091111. 
Okada, S., and Hayashi, T. 1922. Studies on the amino-acid nitrogen content of the blood. J. Biol. Chem. 51:121-133.

Oтто, J. G. 1885. Untersuchungen über die Blutkörperchenzahl und den Hämoglobingehalt des Blutes. (Zweite Abhandlung.) Arch. ges. Physiol. (Pflügers) 36:36-56.

Palmer, L. S., Gortner, R. A., and Rude, R. 1930. The biometry of calcium and inorganic phosphorus in the blood plasma of dairy cattle. Application of results to bone mineralization. J. Dairy Sci. 13:360-367.

Parnas, J. K., and Wagner, R. 1921. Ueber die Ausführung von Bestimmungen kleiner Stickstoffmengen nach Kjeldahl. Biochem. Z. 125:253-256.

Pastore, J. B. 1937. Postpartum hemorrhage. Am. J. Surg. 35:417-429.

Peters, J. P., and Eiserson, L. 1929. The influence of protein and inorganic phosphorus on serum calcium. J. Biol. Chem. 84:155-166.

Peters, J. P., and Van Slyke, D. D. 1931. Quantitative clinical chemistry. Vol. I. Interpretations. The Williams and Wilkins Co., Baltimore.

Pierce, I. H., and Plant, O. H. 1928. Studies in chronic morphine poisoning in dogs. II. Changes in blood cells and hemoglobin during addiction and withdrawal. J. Pharmacol. 33:359-370.

Pilciier, F., JR., and Sireard, C. 1937. Measurements on the loss of blood during transurethral prostatic resection and other surgical procedures determined by spectrophotometric and photelometric methods. Proc. Staff Meetings Mayo Clinic 12:209-213.

Plescir, J. 1909. Hämodynamische Studien. I. Die Blutmenge. Z. exptl. Path. Therap. 6:380-389.

Pommerenke, W. T., Slavin, H. B., Kariher, D. H., and Wiimple, G. H. 1935. Blood plasma protein regeneration controlled by diet. Systematic standardization of food proteins for potency in protein regeneration; fasting and iron feeding. Dog plasma protein given by vein utilized in body metabolism of dog; horse plasma and dog hemoglobin not similarly utilized. J. Exptl. Med. 61:261-282; 283-297.

Ponder, E., and Millar, W. G. 1928. The measurement of the diameters of erythrocytes. III. Changes in the diameter in anaemia resulting from haemorrhage. Quart. J. Exptl. Physiol. 19:145-160.

Powers, J. H., Bowie, M. A., and Howard, I. M. 1930. Some observations on the blood of normal dogs, with special reference to the total volume. Am. J. Physiol. 92:665-671.

Pregl, F. 1924. Quantitative organic micro-analysis, translated by Ernest Fyleman. P. Blakiston's Sons and Co., Philadelphia.

Price-Jones, C. 1922. The diameters of red cells in pernicious anemia and in anemia following hemorrhage. J. Path. Bact. 25:487-504.

. 1932. The red cells in microcytic anemia (Witts). J. Path. Bact. $35: 759-767$.

Randles, F. S., and Knudson, A. 1922. The estimation of lipoid phosphoric acid ("lecithin") in blood by application of the Bell and Doisy method for phosphorus. J. Biol. Chem. 53:53-59.

Reckzer, P. 1904. Ueber die durch das Alter der Organismen bedingten Verschiedenheiten der experimentelle erzeugten Blutgiftanämieen. Z. klin. Med. $54: 165-196$.

Robertson, O. H., and Bock, A. V. 1919. Blood volume in wounded soldiers. I. Blood volume and related blood changes after hemorrhage. II. The use of forced fluids by the alimentary tract in the restoration of blood volume after hemorrhage. J. Exptl. Med. 29:139-153; 155-171. 
RoE, J. H., and KaHn, B. S. 1929. The colorimetric determination of blood calcium. J. Biol. Chem. 81:1-8.

Rowntree, L. G., Broivn, G. E., and Roth, G. M. 1929. The volume of blood and plasma in health and disease. W. B. Saunders Co., Philadelphia.

Scarborougir, R. A. 1930-31. The blood picture of normal laboratory animals. Yale J. Biol. Med. 3:359-373; 4:328.

Schauman, O., and Rosenquist, E. 1898. Ueber die Natur der Blutveränderungen im Höhenklima. Z. klin. Med. 35:126-170.

Sciieunert, A., and von Pelcinzim, H. 1923. Ueber den Gehalt des Blutes verschiedener Tierarten an Zucker, Rest-N, Harnstoff-N, Kreatininkörpern und Harnsäure nach den Folinschen Methoden. Biochem. Z. 139:17-29.

Schittenielm, A., Weichardt, W., and Grisshammer, W. 1911-12. Eiweissumsatz und Ueberempfindlichkeit I Mitteilung. Ueber den Einfluss parenteral verabreichter Proteinsubstanzen verschiedenster Herkunft auf das Blutbild. Z. exptl. Path. Therap. 10:412-447.

Schlutz, F. W., Sivanson, W. W., and Ziegler, Mildred. 1928. Experimental study of plasma protein regeneration. J. Biol. Chem. 78: Sci. Proc., pp. vii-viii.

Scirmidt, C. 1848. Die Diagnostik verdächtiger Flecke in Criminalfällen. Leipzig. (Quoted by Scarborough.)

Scinmidт, H. 1878. Ueber die Möglichkeit der Unterscheidung menschlichen und thierischen Blutes in trockenen Flecken in gerichtlich medizinischer Beziehung. Diss. Erlangen. (Quoted by Scarborough.)

Sexiroo, T. 1929-30. Ueber den Einfluss der Gallensäure auf den Calcium-Stoffwechsel. J. Biochem. (Japan) 11:391-406.

Selinoff, A. G. 1904. Sur les globules blancs pendant l'écoulement au-dehors de la lymphe de la portion cervicale du canal thoracique. Arch. sci. biol. St. Petersburg 10:273-292.

Setir, T. N., and Luck, J. M. 1925. The relation between the metabolism and the specific dynamic action of amino-acids. Biochem. J. 19:366-376.

Sirafrer, P. A. 1914. Observations on creatine and creatinine. J. Biol. Chem. 18: 525-540.

Sherrington, C. S. 1894. Note on some changes in the blood of the general circulation consequent upon certain inflammations of acute and local character. Proc. Roy. Soc. (London) 55:161-207.

Sherrington, C. S., and Copeman, S. M. 1893. Variations experimentally produced in the specific gravity of the blood. J. Physiol. 14:52-96.

Smirk, F. H. 1928. I. The accurate measurement of the proportion of corpuscles and serum in blood. II. The volume of red blood corpuscles in venous and oxygenated blood and after exposure to various saturations of carbon dioxide. Brit. J. Exptl. Path. 9:81-89.

Smitir, H. P., Arnold, H. R., and Wiripple, G. H. 1921. Blood volume studies. VII. Comparative values of Welcker, carbon monoxide, and dye methods for blood volume determinations. Accurate estimation of absolute blood volume. Am. J. Physiol. 56:336-360.

Smiti, H. P., Belt, A. E., and Whipple, G. H. 1920. I. Rapid blood plasma protein depletion and the curve of regeneration. Am. J. Physiol. 52:54-71.

Smitr, M. C., and Otrs, L. 1937. Sex variations in the utilization of iron by anemic rats. Science $85: 125-126$.

Smith, R. C., and Sternberger, H. R. 1932. Diffusible and non-diffusible blood serum calcium following intravenous injection of calcium salts. J. Biol. Chem. 96:245-257. 
Strauss, M. B., and Castle, W. B. 1933. Studies of anemias in pregnancy. III. Am. J. Med. Sci. 185:539.

Stucky, C. J., and Rose, W. B. 1929. Studies in the physiology of vitamins. VII. Hemoglobin, solids, sugar, and chloride changes in the blood of vitamin B deficient dogs. Am. J. Physiol. 89:1-17.

Sturgis, C. C. 1938. Diseases associated with characteristic changes in the red blood cells in microcytic and macrocytic anemias. J. Iowa State Med. Soc. 28:373-379.

Sussdorf, M. 1890. Ellenberger's Handbuch der vergleichenden Physiologie der Haussäugethiere. (Quoted by Scarborough.)

Tallovist, T. W:, and von Willebrand, E. A. 1899-1900. Zur Morphologie der weissen Blutkörperchen des Hundes und des Kaninchens. Skand. Arch. Physiol. 10:37-52.

Taylor, A. E., and Lewis, H. B. 1915a. A study of the protein metabolism under conditions of repeated hemorrhage. J. Biol. Chem. 22:71-75.

. 1915b." On the predominance of the liver in the formation of urea. J. Biol. Chem. $22: 77-80$.

TeEL, H. M., and Watkins, O. 1929. The effect of extracts containing the growth principle of the anterior hypophysis upon the blood chemistry of dogs. Am. J. Physiol. 89:662-685.

Tochowicz, Leon. 1936-37. Recherches sur la fluctuation du taux des acids aminés chez les sujets atteints d'anémie pernicieuse de Biermer et chez les sujets atteints d'anémie posthémorragique. Folia Haematol. 56:249-268.

Turner, Mary E., and Gibson, R. B. 1932. A study of the protein-lipid combinations in blood and body fluids. I. Normal human and dog plasma and horse serum. J. Clin. Investigation 11:735-746.

VAnderhoff, D., and Davis, D. 1932. Anemia of the microcytic type in middleaged women. Am. J. Med. Sci. 184:29-32.

Van Slyke, D. D. 1913-14. The gasometric determination of aliphatic amino nitrogen in minute quantities. J. Biol. Chem. 16:121-124.

1917. Studies of acidosis. II. A method for the determination of carbon dioxide and carbonates in solution. J. Biol. Chem. 30:347-368.

Van Slyke, D. D., and Meyer, G. M. 1912. The amino-acid nitrogen of the blood. Preliminary experiments on protein assimilation. J. Biol. Chem. 12:399410.

1913-14a,b. The fate of protein digestion products in the body. III. The absorption of amino-acids from the blood by the tissues. IV. The locus of chemical transformation of absorbed amino-acids. J. Biol. Chem. $16: 197-212 ; 213-229$.

Van Slyke, D. D., and Neill, J. M. 1924. The determination of gases in blood and other solutions by vacuum extraction and manometric measurement. I. J. Biol. Chem. 61:523-573.

VARS, H. M. 1930. Blood fibrin studies: The concentration of fibrin yielded by canine plasma in relation to dietary factors. Am. J. Physiol. 93:554-567.

Vierordt, K. 1854. (Quoted by Scarborough.)

Weech, A. A., Goetrsch, E., and Reeves, E. B. 1935. Nutritional edema in the dog. I. Development of hypoproteinemia on a diet deficient in protein. J. Expt1. .Med. 61:299-317.

Weech, A. A., Wollstein, M., and Goetrsch, E. 1937. Nutritional edema in the dog. V. Development of deficits in erythrocytes and hemoglobin on a diet deficient in protein. J. Clin. Investigation 16:719-728. 
WeLCKer, H. 1863-64. Grösse, Zahl, Volum, Oberfläche, und Farbe der Blutkörperchen bei Meneschen und bei Thieren. Z. rat. Med., ser. 3, 19-20:257-307.

Wells, J. J., and Sutton, J. E., JR. 1915-16. Blood counts in the frog, the turtle, and twelve different species of mammals. Am. J. Physiol. 39:31-36.

Whipple, G. H. 1914. Fibrinogen. I. An investigation concerning its origin and destruction in the body. Am. J. Physiol. 33:50-69.

Winipple, G. H., Hooper, C. W., and Robscheit, F. S. 1920. Blood regeneration following simple anemia. I. Mixed diet reaction. II. Fasting compared with sugar feeding; analysis of "sparing action of carbohydrates." Am. J. Physiol. 53:151-166; 167-205.

Whipple, G. H., and Robscheit, F. S. 1921. Iron and arsenic as influencing blood regeneration following simple anemia. VI. Negative influence of familiar drugs on the curve of hemoglobin regeneration following hemorrhage. Arch. Internal Med. 27:591-603.

Whipple, G. H., and Robscheit-Robbins, F. S. 1930. Blood regeneration in severe anemia. XVI. Optimum iron therapy and salt effect. Am. J. Physiol. 92: 362-377.

Whipple, G. H., Robscheit, F. S., and Hooper, C. W. 1920. Blood regeneration following simple anemia. IV. Influence of meat, liver, and various extractives, alone or combined with standard diets. Am. J. Physiol. 53: 236-262.

Whipple, G. H., Smith, H. P., and Belt, A. E. 1920. II. Shock as a manifestation of tissue injury following rapid plasma protein depletion. The stabilizing value of plasma proteins. Am. J. Physiol. 52:72-100.

Wilson, D. W., and Plass, E. D. 1917. Creatine and creatinine in whole blood and plasma. J. Biol. Chem. 29:413-423.

Wintrobe, M. M. 1929-30. Classification of the anemias on the basis of differences in the size and hemoglobin content of the red corpuscles. Proc. Soc. Exptl. Biol. Med. 27:1071-1073.

1931. The direct calculation of the volume and hemoglobin content of the erythrocyte. A comparison with color index, volume index, and saturation index determinations. Am. J. Clin. Path. 1:147-165.

1934. Anemia classification and treatment on the basis of differences in the average volume and hemoglobin content of the red corpuscles. Arch. Internal Med. 54:256-280.

Wintrobe, M. M., and Beebe, R. T. 1933. Idiopathic hypochromic anemia. Medicine $12: 187-243$.

Wintrode, M. M., Scirumacker, H. B., Jr., and Schmidt, W. J. 1935-36. Values for number, size, and hemoglobin content of erythrocytes in normal dogs, rabbits, and rats. Am. J. Physiol. 114:502-507.

Witrs, L. J. 1931. Chronic microcytic anemia. Brit. Med. J. 2:883-888.

Woon, I. J. 1936. Treatment of haemorrhage. Brit. Med. J. 2:115-121.

WrIGHT, C. B. 1933. Chronic microcytic anemia, its relationship to menstrual disturbance and achlorhydria. Northwest Medicine 32:1-7.

WU, H. 1922. A new colorimetric method for the determination of plasma proteins. J. Biol. Chem. 51:33-39.

Youngburg, G. E., and Youngburg, M. V. 1936. Phosphorus metabolism. VI. Changes and relationships in blood phosphorus of rats subjected to blood regeneration by repeated bleedings. J. Lab. Clin. Med. 21:798-808.

Zenoni, C. 1894. Ueber die Entstehung der verschiedenen Leukocytenformen des Blutes. Beitr. path. Anat. 16:537-551. 


\section{APPENDIX}

Table I. Physical and Chemical Measurements on

\begin{tabular}{|c|c|c|c|c|c|c|c|c|}
\hline & & $\begin{array}{c}\text { Pre-or } \\
\text { post- } \\
\text { hemor- } \\
\text { rhagic } \\
\text { time } \\
\text { interval }\end{array}$ & $\begin{array}{c}\text { Blood } \\
\text { re- } \\
\text { moved, } \\
\text { in } \\
\text { cc. }\end{array}$ & $\begin{array}{c}\text { Total } \\
\text { blood } \\
\text { volume, } \\
\text { in cc. } \\
\text { per kg. }\end{array}$ & $\begin{array}{c}\text { Cell } \\
\text { volume, } \\
\text { in cc. } \\
\text { per kg. }\end{array}$ & $\begin{array}{l}\text { Cell } \\
\text { vol- } \\
\text { ume } \\
\text { per } \\
\text { cent }\end{array}$ & $\begin{array}{c}\text { Red } \\
\text { cell } \\
\text { count, } \\
\text { in } \\
\text { millions }\end{array}$ & $\begin{array}{l}\text { Mean } \\
\text { corpus- } \\
\text { cular } \\
\text { volume, } \\
\text { incubic } \\
\text { microns }\end{array}$ \\
\hline 1 & Pre-hemorrhage & 7 days & 75 & 74.2 & 34.1 & 45.9 & 7.33 & 62.6 \\
\hline 2 & & 6 days & 74 & 77.5 & 33.0 & 42.6 & 7.01 . & 60.8 \\
\hline 3 & & 4 days & 83 & 68.1 & 28.5 & 41.9 & 7.16 & 58.6 \\
\hline 4 & & 2 days & 69 & 67.5 & 26.8 & 39.7 & 7.04 & 56.4 \\
\hline 5 & & 1 day & 28 & 75.8 & 29.2 & 38.6 & $\ldots \ldots \ldots \ldots$ & .............. \\
\hline 6 & . & same day & 10 & $\ldots \ldots \ldots$ & $\ldots \ldots \ldots$ & $\ldots \ldots \ldots . . .$. & $\ldots$ & ............... \\
\hline 7 & First Hemorrhage & & 301 & .............. & ............... & 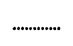 & ............... & ................... \\
\hline 8 & Post-hemorrhage & $30 \mathrm{~min}$. & 55 & 62.5 & 20.6 & 33.0 & 5.48 & 60.2 \\
\hline 9 & & 1 day & 84 & 67.8 & 21.1 & 31.2 & 5.24 & 59.6 \\
\hline 10 & & 2 days & 73 & 66.1 & 18.6 & 28.4 & 5.12 & 55.5 \\
\hline 11 & & 3 days & 70 & 62.7 & 16.3 & 26.0 & 4.64 & 56.0 \\
\hline 12 & & 5 days & 71 & 62.8 & 16.8 & 26.8 & 4.83 & 55.5 \\
\hline 13 & & 6 days & 72 & 62.1 & 13.9 & 22.4 & 3.90 & 57.4 \\
\hline 14 & & 14 days & 70 & 68.0 & 21.3 & 31.4 & 4.78 & 65.6 \\
\hline 15 & Pre-hemorrhage & 13 days & 76 & $\ldots \ldots \ldots \ldots$ & $\ldots \ldots \ldots \ldots . . .$. & $\ldots \ldots \ldots . . . .$. & 6.74 & $\ldots \ldots \ldots \ldots . .$. \\
\hline 16 & & 12 days & 75 & 70.1 & 27.0 & 38.6 & 6.74 & 57.3 \\
\hline 17 & & 11 days & 71 & 74.2 & 27.4 & 36.9 & 6.54 & 56.5 \\
\hline 18 & & 10 days & 72 & 74.6 & 26.4 & 35.2 & 6.26 & 56.2 \\
\hline 19 & & same day & 12 & 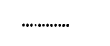 & $\ldots \ldots \ldots \ldots . .$. & 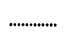 & $\ldots+\cdots \cdots \cdots \cdot \cdots$ & $\ldots+\cdots \cdots \cdots \cdot \cdots$ \\
\hline 20 & Second Hemorrhage & & 313 & 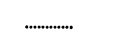 & $\ldots \ldots \ldots . . .$. & $\ldots \ldots \ldots$ & 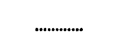 & $\ldots$ \\
\hline 21 & Post-hemorrhage & $30 \mathrm{~min}$. & 63 & 56.8 & 19.6 & 34.5 & 5.54 & 62.2 \\
\hline 22 & & 1 day & 75 & 65.9 & 20.5 & 31.2 & $5.10^{\circ}$ & 61.1 \\
\hline 23 & & 2 days & 72 & 65.5 & 20.1 & 30.7 & 4.68 & 65.6 \\
\hline 24 & & 3'days & 73 & 63.7 & 17.1 & 26.9 & 4.56 & 58.9 \\
\hline 25 & & 4 days & 74 & 67.7 & 19.4 & 28.7 & 4.96 & 57.8 \\
\hline 26 & & 6 days & 74 & 65.5 & 16.6 & 25.3 & 4.16 & 60.9 \\
\hline 27 & & 23 days & 77 & 64.1 & 20.9 & 32.7 & 5.42 & 60.3 \\
\hline 28 & & 24 days & 78 & 70.3 & 22.3 & 31.8 & 5.08 & 62.7 \\
\hline
\end{tabular}


Dog No. $44 \delta َ$ (Mean Weight, 22.9 Kilograms)

\begin{tabular}{|c|c|c|c|c|c|c|c|c|c|}
\hline $\begin{array}{l}\text { Oxygen- } \\
\text { combining } \\
\text { capacity, } \\
\text { in } \\
\text { volumes } \\
\text { per cent }\end{array}$ & $\begin{array}{l}\text { White } \\
\text { cell } \\
\text { count, } \\
\text { in } \\
\text { thou- } \\
\text { sands }\end{array}$ & $\begin{array}{c}\text { Non: } \\
\text { protein } \\
\text { nitrogen } \\
\text { in plasma, } \\
\text { g.per } \\
100 \text { cc. }\end{array}$ & $\begin{array}{c}\text { Protein } \\
\text { nitrogen } \\
\text { in plasma, } \\
\text { g. per } \\
100 \mathrm{cc} .\end{array}$ & $\begin{array}{l}\text { Total } \\
\text { phos- } \\
\text { phorus in } \\
\text { plasma, } \\
\text { mg. per } \\
100 \text { cc. }\end{array}$ & $\begin{array}{l}\text { Lipide } \\
\text { phos- } \\
\text { phorus in } \\
\text { plasma, } \\
\text { mg. per } \\
100 \text { cc. }\end{array}$ & $\begin{array}{l}\text { Inorganic } \\
\text { phos- } \\
\text { phorus in } \\
\text { plasma, } \\
\text { mg. per } \\
\text { loo cc. }\end{array}$ & $\begin{array}{l}\text { Diffusible } \\
\text { calcium } \\
\text { in } \\
\text { plasma, } \\
\text { mg. per } \\
100 \text { cc. }\end{array}$ & \multicolumn{2}{|c|}{$\begin{array}{l}\text { Non- } \\
\text { diffusible } \\
\text { calcium } \\
\text { in plasma, } \\
\text { mg. per } \\
100 \text { cc. }\end{array}$} \\
\hline 16.5 & 7.41 & 0.026 & 1.111 & 26.0 & 17.2 & 5.75 & 8.17 & 5.34 & 1 \\
\hline 15.8 & 6.09 & 0.023 & 1.048 & 25.8 & 17.1 & 6.27 & 8.07 & 5.65 & 2 \\
\hline 16.6 & 8.38 & 0.022 & 1.053 & 26.1 & 17.3 & 5.83 & 8.05 & 4.94 & 3 \\
\hline 15.6 & 7.20 & 0.020 & 1.062 & 26.4 & 19.5 & 5.54 & 8.37 & 4.62 & 4 \\
\hline$\ldots . . . . . . . .$. & $\ldots$ & 0.021 & 1.040 & $\ldots \ldots \ldots \ldots$ & $\ldots$ & $\ldots+\ldots . .$. & $\ldots . . . . . .$. & $\ldots$ & 5 \\
\hline$\ldots$ & ............. & $\ldots$ & $\ldots$ & 26.8 & 18.9 & 6.49 & 8.82 & 5.44 & 6 \\
\hline ................ & 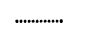 & ........................ & $\ldots$ & ..................... & .............. & ................ & ....................... & ....................... & 7 \\
\hline 13.9 & 5.08 & 0.022 & 0.986 & 24.7 & 18.6 & 6.01 & 8.16 & 5.93 & 8 \\
\hline 12.0 & 6.24 & 0.020 & 1.049 & 23.9 & 18.4 & 5.79 & 6.82 & 6.83 & 9 \\
\hline 10.9 & 6.64 & 0.019 & 1.001 & 25.0 & 18.8 & 6.53 & 7.66 & $\ldots . . . . . . .$. & 10 \\
\hline 10.8 & 6.01 & 0.019 & 0.978 & 23.8 & 16.6 & 5.67 & 8.26 & 3.86 & 11 \\
\hline 10.7 & 6.54 & 0.019 & 1.003 & 23.8 & 17.2 & 5.96 & 8.57 & 4.73 & 12 \\
\hline 9.8 & 5.99 & 0.020 & 0.959 & 24.2 & 19.0 & 5.90 & 7.83 & 6.12 & 13 \\
\hline 12.3 & 10.16 & 0.023 & 1.099 & 25.2 & 15.9 & 7.02 & 8.02 & 4.23 & 14 \\
\hline 15.2 & 8.04 & 0.021 & 1.094 & 26.9 & 18.2 & 6.99 & 7.64 & 4.36 & 15 \\
\hline 14.6 & 12.46 & 0.020 & 1.119 & 22.6 & 16.5 & 5.26 & 8.48 & 4.89 & 16 \\
\hline 15.5 & 9.52 & 0.021 & 1.119 & 24.6 & 17.5 & 5.32 & 7.38 & 4.24 & 17 \\
\hline 14.0 & 8.89 & 0.019 & 1.085 & 24.6 & 17.8 & 5.29 & 7.31 & 4.20 & 18 \\
\hline$\ldots$ & ;...................... & ..................... & ..................... & 23.5 & 17.8 & 5.76 & 6.29 & 4.80 & 19 \\
\hline 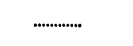 & ...................... & $\ldots$ & ...................... & $\ldots$ & ............... & $\ldots \ldots \ldots \ldots . .$. & .................... & $\ldots \ldots \ldots \cdots$ & 20 \\
\hline 14.8 & 5.90 & 0.020 & 1.060 & 22.5 & 15.5 & 5.36 & 6.03 & 5.42 & 21 \\
\hline 13.2 & 6.99 & 0.018 & 1.025 & 24.9 & 17.6 & 6.13 & 6.27 & 4.90 & 22 \\
\hline 12.2 & 7.45 & 0.018 & 1.026 & 23.8 & 17.9 & 5.93 & 6.11 & 6.37 & 23 \\
\hline 10.7 & 6.21 & 0.017 & 0.979 & 22.5 & 15.0 & 5.43 & 5.98 & 5.47 & 24 \\
\hline 11.3 & 6.21 & 0.018 & 0.981 & 22.4 & 16.7 & 5.62 & 6.29 & 3.80 & 25 \\
\hline 9.8 & 5.55 & 0.018 & 0.993 & 22.3 & 16.6 & 5.36 & 6.19 & 4.52 & 26 \\
\hline 12.4 & 6.42 & 0.022 & 1.111 & 24.2 & 17.9 & 6.53 & 6.89 & 6.17 & 27 \\
\hline 11.9 & 8.29 & 0.023 & 1.116 & 22.9 & 18.7 & 5.45 & 6.28 & 6.32 & 28 \\
\hline
\end{tabular}


Table II. Physical and Chemical Measurements on

\begin{tabular}{|c|c|c|c|c|c|c|c|c|}
\hline & & $\begin{array}{c}\text { Pre-or } \\
\text { post- } \\
\text { hemor- } \\
\text { rhagic } \\
\text { time } \\
\text { interval }\end{array}$ & $\begin{array}{c}\text { Blood } \\
\text { re- } \\
\text { moved, } \\
\text { in } \\
\text { cc. }\end{array}$ & $\begin{array}{c}\text { Total } \\
\text { blood } \\
\text { volume, } \\
\text { in cc. } \\
\text { per kg. }\end{array}$ & $\begin{array}{l}\text { Cell } \\
\text { volume, } \\
\text { in cc. } \\
\text { per kg. }\end{array}$ & $\begin{array}{l}\text { Cell } \\
\text { vol- } \\
\text { ume } \\
\text { per } \\
\text { cent }\end{array}$ & $\begin{array}{c}\text { Red } \\
\text { cell } \\
\text { count, } \\
\text { in } \\
\text { millions }\end{array}$ & $\begin{array}{l}\text { Mean } \\
\text { corpus- } \\
\text { cular } \\
\text { volume, } \\
\text { in cubic } \\
\text { microns }\end{array}$ \\
\hline 1 & Pre-hemorrhage & 35 days & 63 & 83.2 & 44.4 & 53.4 & 7.11 & 75.2 \\
\hline 2 & & 33 days & 65 & 79.0 & 40.2 & 50.9 & 7.85 & 64.8 \\
\hline 3 & & 30 days & 65 & 80.1 & 41.1 & 51.4 & 7.06 & 72.9 \\
\hline 4 & & 28 days & 65 & $\ldots \ldots \ldots . . . .$. & ............... & $\ldots \ldots \ldots . . . .$. & 7.94 & $\ldots \ldots \ldots . . . .$. \\
\hline 5 & & 27 days & 63 & 72.4 & 37.2 & 51.4 & 7.00 & 73.5 \\
\hline 6 & & 9 days & 68 & 81.9 & 43.8 & 53.5 & 7.62 & 70.2 \\
\hline 7 & & 6 days & 72 & 77.6 & 39.9 & 51.8 & 7.98 & 64.9 \\
\hline 8 & & 5 days & 73 & 84.4 & 44.9 & 53.3 & 7.86 & 67.8 \\
\hline 9 & & 2 days & 73 & 78.4 & 38.6 & 49.3 & 7.84 & 62.9 \\
\hline 10 & & same day & 12 & $\ldots+\cdots \cdots \cdots$ & 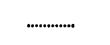 & $\ldots+\cdots \cdots \cdots \cdot \cdots$ & .............. & .................. \\
\hline 11 & First Hemorrhage & & 273 & $\ldots$ & ............... & $\ldots \ldots \ldots \ldots$ & $\ldots$ & ............... \\
\hline 12 & Post-hemorrhage & $30 \mathrm{~min}$. & 53 & $\ldots \ldots \ldots . . . .$. & $\ldots \ldots . . . . . .$. & $\ldots \ldots \ldots \ldots$ & 7.06 & $\ldots \ldots \ldots \ldots . .$. \\
\hline 13 & & $1 \mathrm{~d} \alpha y$ & 63 & 69.7 & 25.7 & 36.9 & 6.48 & 56.9 \\
\hline 14 & & 2 days & 68 & 70.4 & 25.9 & 36.8 & 5.94 & 61.9 \\
\hline 15 & & 3 days & 67 & 69.2 & 23.5 & 33.9 & 5.93 & 57.2 \\
\hline 16 & & 5 days & 66 & 67.3 & 22.7 & 33.8 & 5.42 & 62.3 \\
\hline 17 & & 6 days & 68 & 66.8 & 23.1 & 34.6 & 5.86 & 59.0 \\
\hline 18 & & 8 days & 68 & 68.2 & 21.9 & 32.1 & 5.17 & 62.1 \\
\hline 19 & Pre-hemorrhage & 7 days & 72 & 73.5 & 37.4 & 51.0 & 8.34 & 61.2 \\
\hline 20 & & 6 days & 74 & 74.7 & 36.5 & 48.9 & 8.20 & 59.7 \\
\hline 21 & & 5 days & 75 & .77 .7 & 37.7 & 48.5 & 8.15 & 59.5 \\
\hline 22 & & 4 days & 75 & 77.5 & 36.0 & 46.5 & 8.04 . & 57.8 \\
\hline 23 & & same day & 12 & $\ldots \ldots \ldots+\cdots$ & $\ldots+\cdots \cdots \cdots$ & $\ldots+\cdots \cdots \cdot$ & 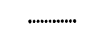 & .................. \\
\hline 24 & Second Hemorrhage & & 250 & $\ldots \ldots \ldots \ldots . .$. & $\ldots \ldots \ldots$ & $\ldots \ldots \ldots \ldots$ & $\ldots \ldots \ldots$ & $\ldots$ \\
\hline 25 & Post-hemorrhage & $30 \mathrm{~min}$. & 63 & 58.5 & 25.5 & 43.8 & 7.27 & 60.2 \\
\hline 26 & & 1 day & 70 & 73.5 & 30.2 & 41.2 & 6.56 & 62.8 \\
\hline 27 & & 2 days & 73 & 65.0 & 22.8 & 35.1 & 6.12 & 57.3 \\
\hline 28 & & 3 days & 67 & 66.5 & 23.6 & 35.5 & 5.61 & 63.3 \\
\hline 29 & & 5 days & 70 & 65.7 & 21.3 & 32.4 & 5.56 & 58.3 \\
\hline 30 & & 7 days & 69 & 67.8 & 22.0 & 32.5 & 5.42 & 60.0 \\
\hline 31 & & 33 days & 72 & 61.7 & 27.8 & 45.1 & 7.19 & 62.7 \\
\hline 32 & & 42 days & 80 & 66.3 & 32.5 & 49.1 & 7.68 & 63.9 \\
\hline
\end{tabular}


Dog No. 45 ơ (Mean Weight, 18.6 Kilograms)

\begin{tabular}{|c|c|c|c|c|c|c|c|c|c|}
\hline $\begin{array}{c}\text { Oxygen- } \\
\text { combining } \\
\text { capacity, } \\
\text { in } \\
\text { volumes } \\
\text { per cent }\end{array}$ & $\begin{array}{l}\text { White } \\
\text { cell } \\
\text { count, } \\
\text { in } \\
\text { thou- } \\
\text { sands }\end{array}$ & $\begin{array}{c}\text { Non- } \\
\text { protein } \\
\text { nitrogen } \\
\text { in plasma, } \\
\text { g. per } \\
100 \text { cc. }\end{array}$ & $\begin{array}{c}\text { Protein } \\
\text { nitrogen } \\
\text { in plasma, } \\
\text { g. per } \\
100 \text { cc. }\end{array}$ & $\begin{array}{l}\text { Total } \\
\text { phos- } \\
\text { phorus in } \\
\text { plasma, } \\
\text { mg. per } \\
\text { loo cc. }\end{array}$ & $\begin{array}{l}\text { Lipide } \\
\text { phos- } \\
\text { phorus in } \\
\text { plasma, } \\
\text { mg. per } \\
100 \text { cc. }\end{array}$ & $\begin{array}{l}\text { Inorganic } \\
\text { phos- } \\
\text { phorus in } \\
\text { plasma, } \\
\text { ma. per } \\
100 \text { cc. }\end{array}$ & $\begin{array}{c}\text { Diffusible } \\
\text { calcium } \\
\text { in } \\
\text { plasma, } \\
\text { mg. per } \\
\text { lo0 cc. }\end{array}$ & $\begin{array}{c}\text { Non- } \\
\text { diffusibl } \\
\text { calcium } \\
\text { in plasm } \\
\text { mg. per } \\
100 \text { cc. }\end{array}$ & \\
\hline 17.5 & 4.08 & 0.032 & 0.907 & 23.0 & 20.9 & 4.36 & $\ldots$ & $\ldots$ & 1 \\
\hline 17.2 & 5.39 & 0.030 & 1.003 & 25.4 & 20.9 & 5.11 & 8.97 & 3.80 & 2 \\
\hline 16.5 & $\begin{array}{l}6.09 \\
5.45\end{array}$ & $\begin{array}{c}0.031 \\
.0 .1\end{array}$ & 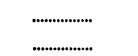 & $\begin{array}{r}24.9 \\
\ldots \ldots \ldots . . .\end{array}$ & 18.1 & 5.02 & 6.64 & 6.71 & $\begin{array}{l}3 \\
4\end{array}$ \\
\hline 18.4 & 4.99 & 0.033 & 1.054 & 23.3 & 20.3 & 5.29 & 7.91 & $\ldots$ & 5 \\
\hline 16.5 & 6.00 & 0.031 & 1.157 & 26.6 & 19.3 & 4.85 & 8.95 & 6.19 & 6 \\
\hline 22.2 & 4.85 & 0.027 & 1.100 & 25.3 & 19.6 & 5.47 & 8.99 & ……….... & 7 \\
\hline 19.5 & 4.50 & 0.031 & 1.144 & 27.6 & 18.9 & 6.36 & 9.57 & 4.90 & 8 \\
\hline 18.8 & 4.75 & 0.032 & 1.089 & 28.5 & 19.8 & 6.75 & 8.98 & 5.85 & 9 \\
\hline .............. & $\ldots . . . . . . . .$. & 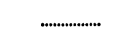 & 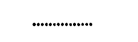 & 28.1 & 20.0 & 6.93 & 8.70 & 6.71 & 10 \\
\hline ............ & 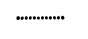 & .................. & 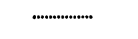 & 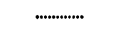 & 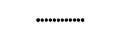 & 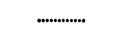 & $\ldots . . . \cdots \cdot \cdots$ & 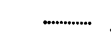 & 11 \\
\hline 18.5 & 3.65 & 0.032 & $\ldots$ & 24.3 & 18.8 & 5.13 & 7.57 & 6.49 & 12 \\
\hline 14.7 & 5.45 & 0.031 & 1.050 & 24.9 & 18.7 & 5.55 & 8.54 & 4.05 & 13 \\
\hline 16.6 & 5.02 & 0.026 & 0.922 & 27.4 & 20.4 & 5.22 & 7.66 & 6.59 & 14 \\
\hline 14.7 & 6.89 & 0.028 & 0.865 & 24.7 & 17.5 & 5.49 & 7.68 & 4.95 & 15 \\
\hline 14.6 & 5.52 & 0.031 & 0.959 & 24.6 & 18.5 & 5.17 & 7.53 & 4.77 & 16 \\
\hline 15.2 & 6.68 & 0.029 & 1.035 & 26.2 & 18.6 & 5.98 & 8.02 & 4.91 & 17 \\
\hline 12.4 & 5.58 & 0.030 & 1.021 & 25.2 & 17.7 & 5.24 & 7.49 & 4.30 & 18 \\
\hline 18.2 & 5.96 & 0.030 & 1.068 & 23.2 & 16.8 & 5.30 & $\ldots$ & ............. & 19 \\
\hline 18.0 & 9.11 & 0.023 & 1.044 & 25.0 & 19.5 & 6.11 & 7.98 & 4.65 & 20 \\
\hline 18.4 & 6.89 & 0.022 & 1.048 & 25.9 & 19.0 & 4.95 & 6.88 & 4.38 & 21 \\
\hline 16.8 & 5.58 & 0.022 & 1.042 & 24.2 & 19.0 & 5.19 & 7.22 & 4.20 & 22 \\
\hline$\ldots$ & ................... & $\ldots$ & $\ldots$ & 23.4 & 18.2 & 5.84 & 7.69 & 4.97 & 23 \\
\hline ............... & $\ldots \ldots+\cdots \cdots \cdots \cdot \cdots$ & ................... & ................... & ............... & .............. & 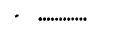 & ............... & $\therefore . . . \cdots \cdots \cdot .$. & 24 \\
\hline 17.7 & 5.02 & 0.020 & 1.034 & 21.0 & 16.1 & 5.05 & 7.50 & 3.56 & 25 \\
\hline 14.0 & 6.08 & 0.024 & ................... & 23.6 & 18.6 & 5.27 & 9.29 & ...................... & 26 \\
\hline 12.6 & 7.34 & 0.022 & 0.945 & 19.0 & 15.0 & 4.65 & 8.14 & 3.19 & 27 \\
\hline 13.6 & 6.86 & 0.022 & 0.959 & 20.6 & 16.8 & 5.46 & 8.31 & 3.21 & 28 \\
\hline 11.6 & 6.55 & 0.023 & 0.959 & 21.2 & 15.3 & 4.95 & 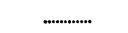 & .............. & 29 \\
\hline 10.9 & 7.16 & 0.025 & 0.958 & 23.1 & 16.0 & 5.28 & 8.37 & 4.58 & 30 \\
\hline 16.7 & 11.41 & 0.025 & 1.080 & 23.7 & 18.3 & 4.87 & 6.96 & 6.93 & 31 \\
\hline 17.9 & 6.48 & 0.030 & 1.069 & 23.5 & 17.2 & 6.03 & 6.48 & 6.93 & 32 \\
\hline
\end{tabular}


Table III. Physical and Chemical Measurements on

\begin{tabular}{|c|c|c|c|c|c|c|c|c|}
\hline & . & $\begin{array}{l}\text { Pre- or } \\
\text { post- } \\
\text { hemor- } \\
\text { rhagic } \\
\text { time } \\
\text { interval }\end{array}$ & $\begin{array}{c}\text { Blood } \\
\text { re- } \\
\text { moved, } \\
\text { in } \\
\text { cc. }\end{array}$ & $\begin{array}{c}\text { Total } \\
\text { blood } \\
\text { volume, } \\
\text { in cc. } \\
\text { per kg. }\end{array}$ & $\begin{array}{l}\text { Cell } \\
\text { volume, } \\
\text { in cc. } \\
\text { per kg. }\end{array}$ & $\begin{array}{l}\text { Cell } \\
\text { vol- } \\
\text { ume } \\
\text { per } \\
\text { cent }\end{array}$ & $\begin{array}{c}\text { Red } \\
\text { cell } \\
\text { count, } \\
\text { in } \\
\text { millions }\end{array}$ & $\begin{array}{l}\text { Mean } \\
\text { corpus- } \\
\text { cular } \\
\text { volume, } \\
\text { in cubic } \\
\text { microns }\end{array}$ \\
\hline 1 & Pre-hemorrhage & 15 days & 72 & 76.2 & 36.6 & 48.1 & 6.64 & 72.4 \\
\hline 2 & & 8 days & 76 & 80.8 & 39.7 & 49.2 & 6.59 & 74.7 \\
\hline 3 & & 4 days & 72 & 84.2 & 39.0 & 46.3 & 6.14 & 75.4 \\
\hline 4 & & 3 days & 74 & 81.3 & 34.2 & 42.1 & 5.52 & 76.3 \\
\hline 5 & & same day & 14 & $\ldots+\cdots \cdots$ & $\ldots$ & $\ldots . . . \cdots \cdots$ & $\ldots . . . \cdots \cdots \cdot . .$. & $\ldots . . . . . . . .$. \\
\hline 6 & First Hemorrhage & & 278 & $\ldots \ldots \ldots \ldots$ & $\ldots . . . . . . .$. & $\ldots \ldots \ldots$ & $\ldots \ldots \ldots$ & $\ldots \ldots \ldots$ \\
\hline 7 & Post-hemorrhage & $30 \mathrm{~min}$. & 60 & 74.0 & 31.8 & 43.0 & 5.53 & 77.8 \\
\hline 8 & & 1 day & 70 & 77.1 & 24.8 & 32.1 & 5.00 & 64.2 \\
\hline 9 & & 2 days & 77 & 72.6 & 22.4 & 31.0 & 4.84 & 64.0 \\
\hline 10 & & 3 days & 76 & 76.4 & 22.2 & 29.1 & 4.61 & 63.1 \\
\hline 11 & & 6 days & 69 & 71.0 & 21.8 & 30.7 & 4.72 & 65.0 \\
\hline 12 & . & 7 days & 70 & 71.4 & 19.8 & 27.7 & 4.48 & 61.9 \\
\hline 13 & & 13 days & 70 & 71.2 & 24.2 & 34.1 & 5.62 & 60.7 \\
\hline 14 & Pre-hemorrhage & 17 days & 74 & 76.2 & 33.3 & 43.7 & 6.76 & 64.6 \\
\hline 15 & & 16 days & 73 & 77.3 & 33.8 & 43.8 & 7.18 & 61.0 \\
\hline 16 & & 7 days & 72 & 79.9 & 33.1 & 41.4 & 6.74 & 61.4 \\
\hline 17 & & 6 days & 100 & 80.8 & 32.2 & 39.9 & 6.34 & 63.0 \\
\hline 18 & & same day & 25 & $\ldots . . . . . . . .$. & $\ldots . . . . . . . . . .$. & $\ldots+\cdots \cdots \cdots$ & .............. & $\ldots . . . \cdots \cdots \cdot . .$. \\
\hline 19 & Second Hemorrhage & & 386 & $\ldots . . . . . . . .$. & 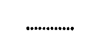 & 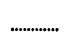 & $\ldots+\ldots . . .$. & $\ldots$ \\
\hline 20 & Post-hemorrhage & $30 \mathrm{~min}$. & 64 & 68.9 & 29.5 & 37.8 & 6.24 & 60.5 \\
\hline 21 & & 1 day & 80 & 65.3 & 20.0 & 30.7 & 4.84 & 63.4 \\
\hline 22 & & 2 days & 75 & 69.7 & 20.8 & 29.9 & 5.38 & 55.6 \\
\hline 23 & & 3 days. & 72 & 68.6 & 17.9 & 26.1 & 4.69 & 55.6 \\
\hline 24 & & 4 days & 74 & 66.7 & 15.3 & 23.0 & 3.90 & 59.0 \\
\hline 25 & & 5 days & 74 & 65.7 & 16.6 & 25.2 & 4.66 & 54.1 \\
\hline 26 & & 29 days & 84 & 72.8 & 24.4 & 33.6 & 5.78 & 58.2 \\
\hline 27 & & 30 days & 76 & 74.3 & 23.0 & 31.0 & 5.90 & 52.6 \\
\hline
\end{tabular}


Dog No. 46 o (Mean Weight, 17.7 Kilograms)

\begin{tabular}{|c|c|c|c|c|c|c|c|c|c|}
\hline $\begin{array}{l}\text { Oxygen- } \\
\text { combining } \\
\text { capacity, } \\
\text { in } \\
\text { volumes } \\
\text { per cent }\end{array}$ & $\begin{array}{l}\text { White } \\
\text { cell } \\
\text { count, } \\
\text { in } \\
\text { thou- } \\
\text { sands }\end{array}$ & $\begin{array}{c}\text { Non- } \\
\text { protein } \\
\text { nitrogen } \\
\text { in plasma, } \\
\text { g. per } \\
100 \text { cc. }\end{array}$ & $\begin{array}{c}\text { Protein } \\
\text { nitrogen } \\
\text { in plasma, } \\
\text { g. per } \\
100 \text { cc. }\end{array}$ & $\begin{array}{l}\text { Total } \\
\text { phos- } \\
\text { phorus in } \\
\text { plasma, } \\
\text { mg. per } \\
\text { lo0 cc. }\end{array}$ & $\begin{array}{l}\text { Lipide } \\
\text { phos- } \\
\text { phorus in } \\
\text { plasma, } \\
\text { mg. per } \\
100 \text { cc. }\end{array}$ & $\begin{array}{l}\text { Inorganic } \\
\text { phos- } \\
\text { phorus in } \\
\text { plasma, } \\
\text { mg. per } \\
\text { loo cc. }\end{array}$ & $\begin{array}{l}\text { Diffusible } \\
\text { calcium } \\
\text { in } \\
\text { plasma, } \\
\text { mg. per } \\
100 \text { cc. }\end{array}$ & $\begin{array}{l}\text { Non- } \\
\text { diffusible } \\
\text { calcium } \\
\text { in plasma } \\
\text { mg. per } \\
100 \text { cc. }\end{array}$ & \\
\hline 19.7 & 11.35 & 0.032 & 1.268 & 22.1 & 12.8 & 6.65 & 8.54 & 5.78 & 1 \\
\hline 19.5 & 7.89 & 0.032 & 1.254 & 21.4 & 12.9 & 6.91 & 7.71 & 5.19 & 2 \\
\hline 17.8 & 9.71 & 0.031 & 1.188 & - 20.6 & 12.9 & 5.53 & 7.90 & $\ldots$ & 3 \\
\hline 17.3 & 9.44 & 0.033 & 1.140 & 21.2 & 11.6 & 5.37 & 7.75 & 5.40 & 4 \\
\hline$\ldots . . \cdots \cdots \cdot \ldots$ & $\ldots . . \cdots \cdots \cdots$ & $\ldots \ldots \ldots \ldots . .$. & $\ldots$ & 20.8 & 13.6 & 5.93 & 7.87 & 4.37 & 5 \\
\hline ............... & 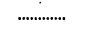 & ...................... & $\ldots \ldots \ldots \ldots \ldots$ & ............... & $\ldots \ldots$ & $\ldots$ & $\ldots$ & $\ldots \ldots \ldots \ldots \ldots$ & 6 \\
\hline 16.0 & 14.04 & 0.028 & 1.104 & 19.0 & 13.2 & 4.71 & 7.82 & 3.49 & 7 \\
\hline 13.4 & 9.54 & 0.024 & 1.064 & 20.3 & 13.0 & 4.46 & 7.80 & 4.68 & 8 \\
\hline 13.0 & 9.39 & 0.024 & 1.072 & 20.2 & 12.6 & 4.99 & 7.93 & 4.58 & 9 \\
\hline 12.5 & 8.34 & 0.027 & 1.038 & 20.4 & 14.3 & 5.08 & 8.19 & 4.28 & 10 \\
\hline 12.3 & 7.78 & 0.025 & 1.007 & 19.9 & 13.2 & 4.96 & 8.45 & 4.21 & 11 \\
\hline 11.5 & 7.71 & 0.028 & 1.025 & 19.5 & 12.5 & 5.00 & 8.35 & 4.49 & 12 \\
\hline 13.4 & 6.98 & 0.027 & 1.027 & 18.8 & 11.9 & 5.24 & 8.71 & 4.62 & 13 \\
\hline 15.4 & 8.14 & 0.029 & 1.015 & 18.4 & 12.6 & 5.15 & 7.05 & 4.34 & 14 \\
\hline 19.1 & 9.31 & 0.027 & 1.117 & 19.1 & 13.1 & 5.24 & ............... & .................. & 15 \\
\hline 15.0 & 7.78 & 0.026 & 1.075 & 18.4 & 11.9 & 5.85 & 7.17 & 4.18 & 16 \\
\hline 15.0 & 6.78 & 0.026 & 1.047 & 18.6 & 12.1 & 5.67 & 7.30 & 3.95 & 17 \\
\hline .................. & $\ldots$ & $\ldots \ldots \ldots \ldots$ & ....................... & 17.0 & 9.7 & 4.51 & 8.45 & 3.54 & 18 \\
\hline$\ldots$ & .............. & 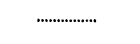 & $\ldots$ & $\ldots \ldots$ & $\ldots \ldots$ & 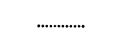 & $\ldots \ldots \ldots$ & $\ldots \ldots \ldots . .$. & 19 \\
\hline 13.6 & 6.06 & 0.027 & 1.005 & 16.7 & 10.6 & 5.10 & 8.58 & 4.47 & 20 \\
\hline 11.3 & 8.72 & 0.022 & 1.005 & 18.1 & 10.6 & 4.49 & 6.66 & 4.09 & 21 \\
\hline 13.1 & 9.59 & 0.025 & 0.982 & 17.4 & 12.6 & 4.62 & 6.82 & 3.92 & 22 \\
\hline 10.6 & 7.59 & 0.028 & 1.032 & 19.4 & 12.6 & 4.86 & 6.25 & 3.81 & 23 \\
\hline 9.1 & 7.79 & 0.024 & 0.970 & 16.6 & 10.2 & 4.21 & 6.69 & 4.48 & 24 \\
\hline 10.9 & 7.05 & 0.024 & 1.027 & 18.4 & 13.3 & 4.68 & 7.06 & 4.57 & 25 \\
\hline 12.1 & 8.49 & 0.023 & 1.112 & 16.4 & 9.4 & 5.55 & 5.86 & 5.50 & 26 \\
\hline 10.2 & 8.14 & 0.026 & 1.111 & 17.4 & ................. & 5.34 & 6.29 & 6.08 & 27 \\
\hline
\end{tabular}


Table IV. Physical and Chemical Measurements on

\begin{tabular}{|c|c|c|c|c|c|c|c|c|}
\hline & & $\begin{array}{l}\text { Pre-or } \\
\text { post } \\
\text { hemor- } \\
\text { rhagic } \\
\text { time } \\
\text { interval }\end{array}$ & $\begin{array}{c}\text { Blood } \\
\text { re- } \\
\text { moved, } \\
\text { in } \\
\text { cc. }\end{array}$ & $\begin{array}{c}\text { Total } \\
\text { blood } \\
\text { volume, } \\
\text { in cc. } \\
\text { per kg. }\end{array}$ & $\begin{array}{l}\text { Cell } \\
\text { volume, } \\
\text { in cc. } \\
\text { per kg. }\end{array}$ & $\begin{array}{l}\text { Cell } \\
\text { vol- } \\
\text { ume } \\
\text { per } \\
\text { cent }\end{array}$ & $\begin{array}{l}\text { Red } \\
\text { cell } \\
\text { count, } \\
\text { in } \\
\text { millions }\end{array}$ & $\begin{array}{l}\text { Mean } \\
\text { corpus- } \\
\text { cular } \\
\text { volume, } \\
\text { in cubic } \\
\text { microns }\end{array}$ \\
\hline 1 & Pre-hemorrhage & 12 days & 68 & 83.0 & 43.3 & 52.3 & 6.86 & 76.2 \\
\hline 2 & & 10 days & 68 & 84.9 & 44.8 & 52.7 & 6.79 & 77.6 \\
\hline 3 & & 6 days & 70 & 77.3 & 38.0 & 49.1 & 6.70 & 73.3 \\
\hline 4 & & 5 days & 63 & 77.4 & 36.5 & 47.2 & 6.60 & 71.5 \\
\hline 5 & & 3 days & 68 & 73.4 & 34.4 & 46.8 & 6.80 & 68.9 \\
\hline 6 & First Hemorrhage & & 248 & .............. & $\ldots$ & ............... & ............... & $\ldots$ \\
\hline 7 & Post-hemorrhage & $30 \mathrm{~min}$. & 68 & 66.7 & 26.2 & 39.3 & 6.23 & 63.1 \\
\hline 8 & & 1 day & 69 & 66.4 & 23.9 & 36.1 & 5.15 & 70.1 \\
\hline 9 & & 2 days & 66 & 56.8 & 19.2 & 33.8 & 5.12 & 66.0 \\
\hline 10 & & 3 days & 66 & 55.9 & 18.3 & 32.8 & 5.32 & 61.6 \\
\hline 11 & & 4 days & 69 & ..................... & ................... & 29.1 & 5.32 & 54.6 \\
\hline 12 & & 7 days & 63 & 67.5 & 23.0 & 34.0 & 5.12 & 66.4 \\
\hline 13 & & 9 days & 65 & 66.9 & 16.7 & 32.9 & 5.62 & 58.5 \\
\hline 14 & Pre-hemorrhage & 21 days & 69 & 77.7 & 34.9 & 44.9 & 7.06 & 63.6 \\
\hline 15 & & 19 days & 69 & 78.5 & 35.1 , & 44.7 & 7.29 & 61.3 \\
\hline 16 & & 17 days & 65 & 70.8 & 28.9 & 40.8 & 7.06 & 57.8 \\
\hline 17 & & 14 days & 68 & 76.1 & 30.6 & 40.2 & 6.99 & 57.5 \\
\hline 18 & & 12 days & 64 & $\ldots \ldots \ldots \ldots$ & .................... & $\ldots . . \cdots \cdots \cdots$ & 6.60 & $\ldots \ldots \ldots . .$. \\
\hline 19 & & 11 days & 64 & 68.6 & 27.1 & 39.6 & 6.44 & 61.5 \\
\hline 20 & & 7 days & 24 & $\ldots . . . . . . .$. & $\ldots . . . . . . .$. & $\ldots \ldots \ldots \ldots . . .$. & $\ldots \ldots \ldots \ldots$ & $\ldots \ldots \ldots$ \\
\hline 21 & & 3 days & 63 & 69.4 & 25.5 & 36.7 & 6.54 & 56.1 \\
\hline 22 & & 2 days & 45 & 74.3 & 28.4 & 38.3 & $\ldots \ldots \ldots \ldots$ & $\ldots . . . . . . . .$. \\
\hline 23 & & same day & 10 & ................... & $\ldots \ldots \ldots$ & .................... & 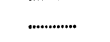 & ............... \\
\hline 24 & Second Hemorrhage & & 306 & $\ldots . . \cdots \cdots \cdots$ & $\ldots+\cdots \cdots \cdots$ & $\ldots+\cdots \cdots$ & $\ldots . . \cdots \cdots \cdots$ & ................... \\
\hline 25 & Post-hemorrhage & $30 \mathrm{~min}$. & 55 & 65.2 & 21.9 & 33.6 & 5.24 & 64.1 \\
\hline 26 & & 1 day & 69 & 63.4 & 17.9 & 28.3 & 4.96 & 57.0 \\
\hline 27 & & 2 days & 66 & 67.2 & 18.7 & 27.8 & 5.32 & 52.2 \\
\hline 28 & & 4 days & 62 & 67.4 & 17.7 & 26.3 & 5.18 & 50.8 \\
\hline 29 & & 5 days & 10 & $\ldots \ldots \ldots . . . . .$. & $\ldots . . . \cdots$ & $\ldots . . . \cdots \cdots . . .$. & $\ldots . . . . . . . . .$. & $\ldots$ \\
\hline 30 & & 7 days & 64 & 68.1 & 18.3 & 26.9 & 4.90 & 55.0 \\
\hline 31 & & 11 days & 70 & 74.4 & 23.9 & 26.9 & 4.96 & 54.2 \\
\hline 32 & Pre-hemorrhage & 5 days & 81 & 72.0 & 28.4 & 39.5 & 8.10 & 48.7 \\
\hline 33 & & 4 days & 69 & 70.4 & 25.2 & 35.9 & 8.60 & 41.7 \\
\hline 34 & & 3 days & 63 & 69.8 & 24.6 & 35.3 & 8.23 & 42.9 \\
\hline 35 & & 2 days & 73 & 71.1 & 24.0 & 33.7 & 7.17 & 47.0 \\
\hline 36 & & same day & 12 & $\ldots \ldots \ldots \ldots$ & $\ldots \ldots \ldots$ & $\ldots \ldots \ldots$ & $\ldots \ldots \ldots$ & $\ldots \ldots \ldots . .$. \\
\hline 37 & Third Hemorrhage & & 303 & $\ldots \ldots \ldots \ldots$ & $\ldots \ldots \ldots \ldots$ & 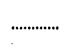 & $\ldots \ldots \ldots \ldots$ & $\ldots \ldots \cdots \cdots$ \\
\hline 38 & Post-hemorrhage & $30 \mathrm{~min}$. & 60 & 55.7 & 16.5 & 29.6 & 6.01 & 49.2 \\
\hline 39 & & 1 day & 65 & 58.2 & 14.8 & 25.4 & 5.81 & 43.7 \\
\hline 40 & & 2 days & 64 & 60.5 & 14.5 & 24.0 & 5.47 & 43.9 \\
\hline 41 & & 3 days & 52 & 58.7 & 15.3 & 26.0 & 5.14 & 50.7 \\
\hline 42 & & 5 days & 62 & 57.6 . & 12.7 & 22.1 & 4.86 & 45.5 \\
\hline 43 & Pre-hemorrhage & 6 days & 75 & 69.6 & 28.2 & 40.6 & 7.76 & 52.4 \\
\hline 44 & & 5 days & 74 & 69.9 & 27.1 & 38.8 & 7.61 & 51.0 \\
\hline 45 & & 4 days & 74 & 69.9 & 25.1 & 35.9 & 7.44 & 48.2 \\
\hline 46 & & 3 days & 74 & 67.8 & 21.8 & 32.2 & 7.06 & 45.6 \\
\hline 47 & & same day & 13 & ............... & $\ldots \ldots \ldots \ldots$ & ............... & $\ldots \ldots \ldots \ldots$ & $\ldots . . \cdots \cdots \cdots \cdot \cdot$ \\
\hline 48 & Fourth Hemorrhage & & 241 & $\ldots+\cdots \cdots \cdots \cdot \cdots$ & $\ldots \ldots \ldots \ldots$ & $\ldots \ldots \ldots$ & $\ldots \ldots \ldots \ldots . \cdots$ & 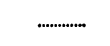 \\
\hline 49 & Post-hemorrhage & $30 \mathrm{~min}$. & 60 & 57.8 & 17.2 & 29.7 & 6.08 & 48.8 \\
\hline 50 & & 1 day & 88 & 70.1 & 19.0 & 27.1 & 5.44 & 49.8 \\
\hline 51 & & 2 days & 74 & 62.6 & 15.6 & 24.5 & 5.69 & 43.1 \\
\hline 52 & & 3 days & 72 & 66.5 & 16.0 & 24.1 & 4.88 & 49.4 \\
\hline 53 & & 4 days & 83 & 65.6 & 15.4 & 23.5 & 4.30 & 54.6 \\
\hline 54 & & 5 days & 72 & 66.5 & 14.0 & 21.1 & 4.86 & 43.4 \\
\hline
\end{tabular}


Dog No. $47 \delta$ (Mean Weight, 18.9 Kilograms)

\begin{tabular}{|c|c|c|c|c|c|c|c|c|c|}
\hline $\begin{array}{l}\text { Oxygen- } \\
\text { combining } \\
\text { capacity, } \\
\text { in } \\
\text { volumes } \\
\text { per cent }\end{array}$ & $\begin{array}{l}\text { White } \\
\text { cell } \\
\text { count, } \\
\text { in } \\
\text { thou- } \\
\text { sands }\end{array}$ & $\begin{array}{c}\text { Non- } \\
\text { protein } \\
\text { nitrogen } \\
\text { in plasma, } \\
\text { g. per } \\
100 \text { cc. }\end{array}$ & $\begin{array}{l}\text { Protein } \\
\text { nitrogen } \\
\text { in plasma, } \\
\text { g. per } \\
100 \text { cc. }\end{array}$ & $\begin{array}{l}\text { Total } \\
\text { phos- } \\
\text { phorus in } \\
\text { plasma, } \\
\text { mg. per } \\
\text { loo cc. }\end{array}$ & $\begin{array}{l}\text { Lipide } \\
\text { phos- } \\
\text { phorus in } \\
\text { plasma, } \\
\text { mg. per } \\
\text { loo cc. }\end{array}$ & $\begin{array}{l}\text { Inorganic } \\
\text { phos- } \\
\text { phorus in } \\
\text { plasma, } \\
\text { mg. per } \\
\text { loo cc. }\end{array}$ & $\begin{array}{l}\text { Diffusible } \\
\text { calcium } \\
\text { in } \\
\text { plasma, } \\
\text { mg. per } \\
100 \text { cc. }\end{array}$ & $\begin{array}{l}\text { Non- } \\
\text { diffusible } \\
\text { calcium } \\
\text { in plasma } \\
\text { mg. per } \\
\text { 100 cc. }\end{array}$ & \\
\hline 17.3 & 7.61 & 0.037 & 1.114 & 22.2 & 16.3 & 4.41 & $\ldots . . . . . . . . .$. & $\ldots \ldots \ldots . . . . .$. & 1 \\
\hline 19.1 & 5.98 & 0.038 & 1.039 & 22.4 & 16.8 & 3.91 & 6.69 & 6.14 & 2 \\
\hline 15.0 & 9.01 & 0.035 & 1.046 & 21.0 & 17.0 & 4.11 & 6.67 & 4.20 & 3 \\
\hline 14.6 & 7.04 & 0.036 & 0.895 & 21.7 & 17.2 & 4.41 & ............... & ................... & 4 \\
\hline 14.0 & 6.34 & 0.034 & 1.062 & 20.5 & 17.3 & 4.04 & 5.93 & 5.39 & 5 \\
\hline ................ & $\ldots \ldots$ & 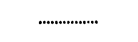 & ....................... & $\ldots$ & $\ldots$ & $\ldots \ldots \ldots \ldots . . .$. & ............... & 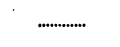 & 6 \\
\hline 11.8 & 7.46 & 0.032 & 0.819 & 17.7 & 14.4 & 3.72 & 6.31 & 5.00 & 7 \\
\hline 12.3 & 8.86 & 0.033 & 0.941 & 21.4 & 18.4 & 4.25 & 6.53 & 6.66 & 8 \\
\hline 13.4 & 7.70 & 0.029 & 0.944 & 20.6 & 16.9 & 4.15 & 6.78 & 4.95 & 9 \\
\hline 12.6 & 8.21 & 0.026 & 0.924 & 26.4 & 20.2 & 3.64 & 5.87 & 4.02 & 10 \\
\hline 11.2 & 7.89 & 0.024 & 0.874 & 22.0 & 18.5 & 4.34 & 6.55 & 4.26 & 11 \\
\hline 14.0 & 9.05 & 0.030 & 0.975 & 22.9 & 17.2 & 4.61 & 6.23 & 4.86 & 12 \\
\hline \multirow[t]{2}{*}{13.6} & 9.00 & 0.031 & 0.962 & 23.7 & 17.1 & 4.74 & 6.43 & 3.03 & 13 \\
\hline & 8.36 & 0.034 & 1.041 & 21.5 & 18.2 & 4.37 & 7.26 & 3.54 & $\overline{14}$ \\
\hline 15.8 & 8.85 & 0.032 & 0.938 & 23.2 & 18.5 & 3.83 & .............. & .............. & 15 \\
\hline 15.2 & 9.18 & 0.032 & 1.030 & 25.8 & 17.6 & 4.64 & 8.48 & 3.49 & 16 \\
\hline \multirow[t]{2}{*}{15.2} & 8.66 & 0.033 & 0.946 & 19.9 & 17.9 & 3.86 & 6.29 & 6.47 & 17 \\
\hline & 11.15 & $\ldots$ & $\ldots$ & $\ldots$ & $\ldots \ldots \ldots \ldots$ & $\ldots$ & $\ldots$ & $\ldots$ & 18 \\
\hline \multirow[t]{2}{*}{12.4} & 9.43 & 0.033 & 0.992 & 24.3 & 18.8 & 4.70 & 7.44 & 3.00 & 19 \\
\hline & & & & 28.6 & 20.6 & 4.38 & 7.56 & 4.20 & 20 \\
\hline \multirow[t]{2}{*}{13.3} & 8.88 & 0.034 & 1.066 & 24.9 & 18.4 & 5.31 & 8.99 & 3.50 & 21 \\
\hline & ............... & $\ldots$ & $\ldots$ & 25.3 & 20.1 & 4.79 & 10.67 & 4.12 & 22 \\
\hline 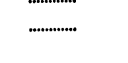 & ............... & ....................... & $\ldots$ & 24.4 & 18.1 & 4.72 & 9.47 & 4.76 & 23 \\
\hline & & & $\ldots$ & $\ldots$ & .................... & $\ldots \ldots \ldots$ & $\ldots$ & .............. & 24 \\
\hline 13.4 & 8.62 & 0.033 & 1.019 & 21.5 & 16.4 & 4.49 & 7.72 & 5.81 & 25 \\
\hline 11.0 & 10.34 & 0.029 & 0.953 & 22.8 & 18.7 & 4.10 & 7.64 & 6.74 & 26 \\
\hline 11.3 & 12.48 & 0.028 & 0.971 & 25.1 & 18.5 & 4.80 & 8.04 & 5.16 & 27 \\
\hline \multirow[t]{2}{*}{11.1} & 10.99 & 0.028 & 1.042 & 23.7 & $\ldots . . . . . . .$. & ............... & 7.30 & ............... & 28 \\
\hline & $\ldots$ & & & 26.1 & 19.6 & 5.54 & 8.29 & 4.53 & 29 \\
\hline 6.5 & 10.44 & 0.030 & 1.024 & 25.1 & 19.2 & 4.79 & 8.99 & 4.32 & 30 \\
\hline 9.3 & 12.01 & 0.026 & 1.041 & 26.6 & 17.4 & 5.48 & $7: 89$ & 4.64 & 31 \\
\hline 10.1 & 6.20 & 0.028 & 1.108 & 25.9 & 22.0 & 4.58 & 7.55 & 6.37 & 32 \\
\hline 14.6 & 12.94 & 0.027 & 1.112 & 25.9 & 19.0 & 5.47 & 8.46 & 4.22 & 33 \\
\hline 13.6 & 8.91 & 0.029 & 1.055 & 29.6 & 19.2 & 6.82 & 7.66 & 3.80 & 34 \\
\hline \multirow{2}{*}{13.0} & 10.06 & 0.027 & 1.072 & 25.8 & 21.3 & 5.01 & 7.37 & 4.27 & 35 \\
\hline & $\ldots$ & $\ldots$ & ....................... & 26.2 & 21.2 & 5.06 & 7.80 & 4.49 & 36 \\
\hline ............... & $\ldots$ & $\ldots$ & $\ldots$ & ...................... & $\ldots+\cdots \cdots$ & $\ldots+\cdots \cdots$ & $\ldots+\cdots \cdots$ & $\ldots+\cdots \cdots \cdots$ & 37 \\
\hline 11.5 & 7.38 & 0.029 & 0.974 & 23.5 & 18.8 & 4.20 & 7.83 & 4.01 & 38 \\
\hline 10.2 & 11.02 & 0.027 & 0.922 & 26.1 & 20.4 & 4.98 & 7.98 & 5.76 & 39 \\
\hline 10.0 & 10.02 & 0.024 & 1.026 & 25.7 & 19.4 & 4.96 & 7.96 & 4.33 & 40 \\
\hline 8.0 & 9.38 & 0.027 & 1.015 & 24.8 & 19.7 & 4.88 & 7.39 & 5.20 & 41 \\
\hline 8.1 & 17.82 & 0.028 & 1.018 & 27.3 & 19.5 & 5.20 & 7.76 & 4.44 & 42 \\
\hline 13.5 & 6.68 & 0.025 & 1.088 & 23.5 & 18.6 & 5.29 & 7.28 & 5.40 & 43 \\
\hline 13.1 & 7.86 & 0.024 & 1.108 & 27.4 & 16.5 & 5.14 & 7.50 & 4.30 & 44 \\
\hline 13.2 & 7.56 & 0.024 & 1.103 & 26.7 & 22.3 & 4.53 & 6.30 & 3.56 & 45 \\
\hline 12.4 & 7.10 & 0.022 & 1.046 & 25.3 & 21.5 & 4.49 & 5.76 & 6.82 & 46 \\
\hline .............. & ............... & ..................... & $\ldots$ & 26.4 & 20.0 & 5.79 & 6.08 & 5.55 & 47 \\
\hline$\ldots$ & .................... & ......................... & ......................... & 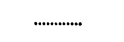 & $\ldots$ & $\ldots \cdots \cdots$ & $\ldots$ & $\ldots \cdots \cdots$ & 48 \\
\hline 12.3 & 7.32 & 0.024 & $\ldots$ & 22.9 & 16.7 & 5.00 & 5.78 & 4.57 & 49 \\
\hline 9.6 & 8.10 & 0.024 & 0.978 & 24.6 & 19.2 & 4.77 & 6.87 & 5.06 & 50 \\
\hline 10.1 & 9.09 & 0.021 & 1.015 & 23.4 & 19.1 & 4.82 & 6.95 & 5.84 & 51 \\
\hline 9.1 & 8.40 & 0.023 & 1.001 & 23.4 & 18.6 & 4.90 & 5.74 & 4.11 & 52 \\
\hline 8.3 & 8.24 & 0.021 & 1.000 & 23.1 & 18.2 & 4.12 & 7.49 & 4.62 & 53 \\
\hline 8.2 & 9.26 & 0.020 & 0.961 & 22.1 & 14.6 & 4.50 & 5.92 & 5.21 & 54 \\
\hline
\end{tabular}


Table V. Physical and Chemical Measurements on Dog No. 20 ㅇ (Mean Weight, 19.3 Kilograms)

\begin{tabular}{|c|c|c|c|c|c|c|c|c|c|c|c|c|c|}
\hline & & $\begin{array}{l}\text { Pre- or } \\
\text { post- } \\
\text { hemor- } \\
\text { rhagic } \\
\text { time } \\
\text { interval }\end{array}$ & $\begin{array}{c}\text { Blood } \\
\text { re- } \\
\text { moved, } \\
\text { in } \\
\text { cc. }\end{array}$ & $\begin{array}{c}\text { Total } \\
\text { blood } \\
\text { volume, } \\
\text { in cc. } \\
\text { per kg. }\end{array}$ & $\begin{array}{c}\text { Cell } \\
\text { volume, } \\
\text { in cc. } \\
\text { per kg. }\end{array}$ & $\begin{array}{l}\text { Cell } \\
\text { vol- } \\
\text { ume } \\
\text { per } \\
\text { cent }\end{array}$ & $\begin{array}{c}\text { Red } \\
\text { cell } \\
\text { count, } \\
\text { in } \\
\text { millions }\end{array}$ & $\begin{array}{l}\text { Mean } \\
\text { corpus- } \\
\text { cular } \\
\text { volume, } \\
\text { in cubic } \\
\text { microns }\end{array}$ & $\begin{array}{l}\text { Oxygen- } \\
\text { combining } \\
\text { capacity, } \\
\text { in } \\
\text { volumes } \\
\text { per cent }\end{array}$ & $\begin{array}{l}\text { White } \\
\text { cell } \\
\text { count, } \\
\text { in } \\
\text { thou- } \\
\text { sands }\end{array}$ & $\begin{array}{c}\text { Nonprotein } \\
\text { nitrogen } \\
\text { in whole } \\
\text { blood, } \\
\text { g. per } \\
100 \mathrm{cc} .\end{array}$ & $\begin{array}{c}\text { Protein } \\
\text { nitrogen } \\
\text { in whole } \\
\text { blood, } \\
\text { g. per } \\
100 \mathrm{cc} .\end{array}$ & \\
\hline 1 & Pre-hemorrhage & 56 days & 22 & 62.2 & 23.6 & 38.0 & 5.65 & 67.3 & $\ldots \ldots \ldots \ldots . .$. & 3.90 & $\ldots \ldots \ldots \ldots \ldots$ & ..................... & 1 \\
\hline 2 & & 14 days & 31 & 65.8 & 30.4 & 46.2 & $\ldots \ldots \ldots \ldots . . .$. & $\ldots \ldots \ldots \ldots . . .$. & $\ldots \ldots \ldots \ldots$ & $\ldots . . . \cdots$ & 0.030 & 2.896 & 2 \\
\hline 3 & & 7 days & 48 & 70.1 & 31.7 & 45.2 & $\ldots \ldots \ldots$ & $\ldots \ldots \ldots \ldots . .$. & 12.4 & $\ldots \ldots \ldots \ldots . . .$. & 0.030 & 2.961 & 3 \\
\hline 4 & & 3 days & 40 & 68.6 & 27.2 & 39.7 & 6.98 & 56.9 & 14.2 & 9.55 & 0.028 & 2.863 & 4 \\
\hline 5 & & same day & 45 & 59.5 & 22.8 & 38.3 & 6.08 & 63.0 & 12.8 & 9.70 & 0.030 & 2.424 & 5 \\
\hline 6 & First Hemorrhage & & 263 & $\ldots \ldots \ldots \ldots . .$. & $\ldots \ldots \ldots \ldots . .$. & $\ldots \ldots \ldots \ldots . .$. & $\ldots \ldots \ldots \ldots . .$. & $\ldots . . . \cdots \cdots . .$. & $\ldots . . . \ldots \ldots . . .$. & $\ldots \ldots+\cdots . . .$. & $\ldots+\ldots+\ldots . . . . .$. & ................. & 6 \\
\hline 7 & & same day & 45 & 71.2 & 22.8 & 32.1 & 5.97 & 53.8 & 13.8 & 7.05 & 0.029 & 2.295 & 7 \\
\hline 8 & Second Hemorrhage & & 228 & $\ldots \ldots \ldots \ldots$ & $\ldots \ldots \ldots \ldots$ & $\ldots \ldots \ldots \ldots . . .$. & $\ldots$ & $\ldots . . \cdots \cdots \cdots$ & $\ldots . . . \cdots \cdots \cdots . .$. & $\ldots \ldots \ldots \ldots$ & $\ldots \ldots \ldots \ldots \ldots$ & $\ldots \ldots \ldots \ldots \ldots$ & 8 \\
\hline 9 & Post-hemorrhage & 2 days & 34 & 59.8 & 19.3 & 32.2 & 5.64 & 57.1 & 9.8 & 5.40 & 0.034 & 2.200 & 9 \\
\hline 10 & & 1 week & 39 & 64.2 & 19.7 & 35.1 & 7.51 & 46.7 & 12.7 & 8.95 & 0.032 & 2.528 & 10 \\
\hline 11 & & 2 weeks & 73 & 56.1 & 14.6 & 26.7 & 4.98 & 53.6 & 11.5 & 7.10 & 0.035 & 2.240 & 11 \\
\hline 12 & & 3 weeks & 40 & 64.6 & 20.4 & 30.7 & 5.85 & 52.5 & 9.1 & 6.55 & 0.028 & 2.011 & 12 \\
\hline 13 & & 4 weeks & 42 & 59.5 & 19.8 & 33.3 & 7.63 & 43.6 & 9.5 & 7.20 & 0.031 & 2.359 & 13 \\
\hline 14 & & 5 weeks & 44 & 59.8 & 17.8 & 30.1 & 5.62 & 53.6 & 7.1 & 9.80 & 0.028 & 2.242 & 14 \\
\hline 15 & & 6 weeks & 44 & 64.0 & 18.9 & 29.5 & 5.66 & 52.1 & 10.7 & 5.30 & 0.029 & 2.080 & 15 \\
\hline 16 & & 7 weeks & 38 & 60.7 & 20.2 & 33.3 & 6.28 & 53.0 & 8.5 & 5.35 & 0.034 & 2.346 & 16 \\
\hline 17 & & 8 weeks & 44 & 69.3 & 26.8 & 38.6 & 6.12 & 63.1 & 8.5 & 5.00 & 0.029 & 2.110 & 17 \\
\hline 18 & & 9 weeks & 49 & 61.0 & 21.7 & 35.5 & 6.41 & 55.4 & 9.4 & 6.95 & 0.033 & 2.352 & 18 \\
\hline 19 & - & 10 weeks & 48 & 63.8 & 21.7 & 34.1 & 8.12 & 42.0 & 10.6 & 6.25 & 0.037 & 2.368 & 19 \\
\hline 20 & & 11 weeks & 47 & 64.8 & 19.3 & 29.7 & 7.00 & 42.4 & 8.0 & 9.05 & 0.028 & 2.582 & 20 \\
\hline 21 & & 12 weeks & 46 & 65.9 & 21.5 & 32.6 & 7.02 & 46.4 & 10.7 & 6.10 & 0.032 & 2.613 & 21 \\
\hline
\end{tabular}


Table VI. Physical and Chemical Measurements on Dog No. 21 \% (Mean Weight, 11.7 Kilograms)

\begin{tabular}{|c|c|c|c|c|c|c|c|c|c|c|c|c|c|}
\hline$\because$ & & $\begin{array}{l}\text { Pre-or } \\
\text { post- } \\
\text { hemor- } \\
\text { rhagic } \\
\text { time } \\
\text { interval }\end{array}$ & $\begin{array}{l}\text { Blood } \\
\text { re- } \\
\text { moved, } \\
\text { in } \\
\text { cc. }\end{array}$ & $\begin{array}{l}\text { Total } \\
\text { blood } \\
\text { volume, } \\
\text { in cc. } \\
\text { per kg. }\end{array}$ & $\begin{array}{c}\text { Cell } \\
\text { volume, } \\
\text { in cc. } \\
\text { per kg. }\end{array}$ & $\begin{array}{l}\text { Cell } \\
\text { vol- } \\
\text { ume } \\
\text { per } \\
\text { cent }\end{array}$ & $\begin{array}{c}\text { Red } \\
\text { cell } \\
\text { count, } \\
\text { in } \\
\text { millions }\end{array}$ & $\begin{array}{l}\text { Mean } \\
\text { corpus- } \\
\text { cular } \\
\text { volume, } \\
\text { in cubic } \\
\text { microns }\end{array}$ & $\begin{array}{c}\text { Oxygen- } \\
\text { combining } \\
\text { capacity, } \\
\text { in } \\
\text { volumes } \\
\text { per cent }\end{array}$ & $\begin{array}{l}\text { White } \\
\text { cell } \\
\text { count, } \\
\text { in } \\
\text { thou- } \\
\text { sands }\end{array}$ & $\begin{array}{l}\text { Nonprotein } \\
\text { nitrogen } \\
\text { in whole } \\
\text { blood, } \\
\text { g. per } \\
100 \text { cc. }\end{array}$ & $\begin{array}{c}\text { Protein } \\
\text { nitrogen } \\
\text { in whole } \\
\text { blood, } \\
\text { g.per } \\
100 \text { cc. }\end{array}$ & \\
\hline 1 & Pre-hemorrhage & 39 days & 30 & 78.9 & 37.2 & 47.1 & $\ldots \ldots \ldots$ & $\ldots . . . . .$. & $\ldots . . . . . . . .$. & ……...... & 0.027 & 3.106 & 1 \\
\hline 2 & & 30 days & 42 & 70.7 & 30.7 & 43.4 & $\ldots \ldots \ldots \ldots . .$. & $\ldots \ldots \ldots \ldots$ & 12.9 & $\ldots \ldots \ldots$ & 0.028 & 3,098 & 2 \\
\hline 3 & & 28 days & 42 & 63.6 & 26.3 & 41.3 & 6.28 & 65.8 & 13.1 & 6.50 & 0.029 & 3.047 & 3 \\
\hline 4 & & 11 days & 41 & 68.5 & 30.9 & 44.8 & 7.08 & 63.3 & 14.7 & 3.25 & 0.026 & 3.140 & 4 \\
\hline 5 & $\because$ & same day & 41 & 56.4 & 27.0 & 48.0 & 7.73 & 62.1 & 14.7 & 18.35 & 0.038 & 3.067 & 5 \\
\hline 6 & First Hemorrhage & & 154 & $\ldots \ldots \ldots \ldots$ & $\ldots \ldots \ldots \ldots \ldots$ & $\ldots \ldots \ldots \ldots \ldots$ & $\ldots \ldots \ldots \ldots . .$. & $\ldots \ldots \ldots \ldots$ & $\ldots . . \cdots \cdots \cdots \cdot$ & $\ldots \ldots \ldots \ldots . .$. & $\ldots \ldots \ldots \ldots \ldots$ & $\ldots \ldots \ldots \ldots \ldots$ & 6 \\
\hline 7 & & same day. & 43 & 58.6 & 22.3 & 38.0 & 6.35 & 59.8 & 12.8 & 17.15 & 0.030 & 2.738 & 7 \\
\hline 8 & Second Hemorrhage & & 152 & $\ldots \cdots \cdots \cdots \cdots$ & $\ldots \ldots \ldots \ldots . .$. & $\ldots \ldots \ldots \ldots$ & $\ldots . . \cdots \cdots \cdots$ & $\ldots . . \cdots \cdots \cdots$ & $\ldots \ldots \ldots \ldots . .$. & $\ldots \ldots \ldots \ldots . .$. & $\ldots \ldots \ldots \ldots \ldots$ & $\ldots \ldots \ldots \ldots \ldots$ & 8 \\
\hline 9 & Post-hemorrhage & 2 days & 41 & 69.4 & 22.1 & 31.9 & 4.80 & 66.4 & 11.6 & 7.00 & 0.035 & 2.480 & 9 \\
\hline 10 & & 1 week & 45 & 63.8 & 25.2 & 39.6 & 7.10 & 55.8 & 13.5 & 13.45 & 0.033 & 2.763 & 10 \\
\hline 11 & & 2 weeks & 45 & 66.3 & 24.0 & 36.2 & 6.64 & 54.5 & 11.3 & 7.15 & 0.028 & 2.457 & 11 \\
\hline 12 & $\cdot$ & 3 weeks & 42 & 55.4 & 23.1 & 42.6 & 7.36 & 57.9 & 12.2 & 4.75 & 0.037 & 2.658 & 12 \\
\hline 13 & & 4 weeks & 44 & 62.2 & 26.8 & 43.0 & 8.15 & 52.8 & 12.0 & 5.40 & 0.030 & 2.741 & 13 \\
\hline 14 & & 5 weeks & 44 & 60.9 & 23.9 & 39.3 & 7.44 & 52.8 & 11.7 & 5.15 & 0.026 & 2.604 & 14 \\
\hline 15 & & 6 weeks & 44 & 63.4 & 25.4 & 40.1 & 8.67 & 46.2 & 10.8 & 10.25 & 0.032 & 2.588 & 15 \\
\hline 16 & & 7 weeks & 47 & 66.6 & 24.8 & 37.5 & 7.90 & 47.5 & 11.2 & 5.60 & 0.025 & 2.445 & 16 \\
\hline 17 & & 8 weeks & 46 & 63.9 & 24.4 & 38.2 & $\ldots \ldots \ldots$ & $\ldots$ & 12.4 & 9.80 & 0.033 & 2.798 & 17 \\
\hline 18 & & 9 weeks & 42 & 56.7 & 22.4 & 39.5 & 8.23 & 48.0 & $\ldots . . . \cdots \cdots$ & $\ldots \ldots \ldots \ldots . .$. & $\ldots . . . . . . .$. & $\ldots . . . \cdots \cdots \cdots$ & 18 \\
\hline
\end{tabular}


Table VII. Physical and Chemical Measurements on Dog No. 22 ㅇ (Mean Weight, 12.9 Kilograms)

\begin{tabular}{|c|c|c|c|c|c|c|c|c|c|c|c|c|c|}
\hline & i & $\begin{array}{l}\text { Pre-or } \\
\text { post- } \\
\text { hemor- } \\
\text { rhagic } \\
\text { time } \\
\text { interval }\end{array}$ & $\begin{array}{c}\text { Blood } \\
\text { re- } \\
\text { moved, } \\
\text { in } \\
\text { cc. }\end{array}$ & $\begin{array}{c}\text { Total } \\
\text { blood } \\
\text { volume, } \\
\text { in cc. } \\
\text { per kg. }\end{array}$ & $\begin{array}{c}\text { Cell } \\
\text { volume, } \\
\text { in cc. } \\
\text { per kg. }\end{array}$ & $\begin{array}{l}\text { Cell } \\
\text { vol- } \\
\text { ume } \\
\text { per } \\
\text { cent }\end{array}$ & $\begin{array}{l}\text { Red } \\
\text { cell } \\
\text { count, } \\
\text { in } \\
\text { millions }\end{array}$ & $\begin{array}{l}\text { Mean } \\
\text { corpus- } \\
\text { cular } \\
\text { volume, } \\
\text { in cubic } \\
\text { microns }\end{array}$ & $\begin{array}{l}\text { Oxygen- } \\
\text { combining } \\
\text { capacity. } \\
\text { in } \\
\text { volumes } \\
\text { per cent }\end{array}$ & $\begin{array}{l}\text { White } \\
\text { cell } \\
\text { count, } \\
\text { in } \\
\text { thou- } \\
\text { sands }\end{array}$ & $\begin{array}{c}\text { Nonprotein } \\
\text { nitrogen } \\
\text { in whole } \\
\text { blood, } \\
\text { g. per } \\
100 \mathrm{cc} .\end{array}$ & $\begin{array}{c}\text { Protein } \\
\text { nitrogen } \\
\text { in whole } \\
\text { blood, } \\
\text { g. per } \\
100 \mathrm{cc} .\end{array}$ & \\
\hline 1 & Pre-hemorrhage & 12 days & 41 & 61.8 & 28.1 & 45.4 & 6.18 & 73.5 & 18.5 & 4.15 & 0.039 & 2.812 & 1 \\
\hline 2 & & same day & 41 & 51.5 & 23.1 & 44.9 & 7.35 & 61.1 & 16.2 & 10.45 & 0.035 & 2.924 & 2 \\
\hline 3 & First Hemorrhage & & 159 & $\ldots . . . \cdots \cdots \cdot . .$. & $\ldots . . \cdots \cdots \cdots \cdot \cdot \cdot$ & $\ldots . . . \cdots \cdots \cdot . \cdot$ & $\ldots . . . \cdots \cdots \cdot . .$. & $\ldots . . \cdots \cdots \cdots . .$. & $\because \cdots \cdots \cdots$ & .............. & .................. & ................. & 3 \\
\hline 4 & & same day & 43 & 55.0 & 18.8 & 34.2 & 5.54 & 61.7 & 12.7 & 9.40 & 0.035 & 2.420 & 4 \\
\hline 5 & Second Hemorrhage & & 163 & $\ldots \ldots \ldots \ldots$ & .............. & ............... & .............. & $\ldots . . \cdots \cdots \cdots .$. & $\ldots . . . \cdots \cdots \cdot \cdot \cdot$ & $\ldots . . . \cdots \cdots \cdot \cdots$ & $\ldots . \cdots \cdots \cdots \cdots \cdots \cdots \cdot \cdots$ & $\ldots \ldots \ldots \ldots \ldots . \cdots$ & 5 \\
\hline 6 & Post-hemorrhage & 3 days & 42 & 59.4 & 20.8 & 34.9 & 4.54 & 76.9 & 10.4 & 10.20 & 0.053 & 2.272 & 6 \\
\hline 7 & & 1 week & 38 & 50.7 & 17.6 & 34.7 & 4.89 & 71.0 & 9.2 & 15.60 & 0.052 & 2.297 & 7 \\
\hline 8 & & 2 weeks & 47 & 58.3 & 22.3 & 38.2 & 6.75 & 56.6 & 9.9 & 13.45 & 0.040 & 2.445 & 8 \\
\hline 9 & & 3 weeks & 47 & 58.6 & 21.9 & 37.3 & 6.01 & 62.1 & 8.8 & 9.25 & 0.035 & 2.525 & 9 \\
\hline 10 & & 4 weeks & 45 & 57.1 & 22.8 & 40.0 & 6.76 & 59.2 & 10.7 & 7.30 & 0.038 & 2.562 & 10 \\
\hline 11 & & 5 weeks & 46 & 61.0 & 26.9 & 44.1 & 7.54 & 58.5 & 11.6 & 8.80 & 0.047 & 2.694 & 11 \\
\hline 12 & & 6 weeks & 45 & 60.7 & 28.7 & 47.3 & 7.02 & 67.4 & 11.0 & 4.50 & 0.042 & 2.668 & 12 \\
\hline 13 & & 7 weeks & 45 & 59.3 & 29.6 & 50.0 & 7.89 & 63.4 & 12.8 & 6.05 & 0.049 & 2.859 & 13 \\
\hline 14 & & 8 weeks & 46 & 60.3 & 26.0 & 43.2 & 7.27 & 59.4 & 11.9 & 6.60 & 0.047 & 2.724 & 14 \\
\hline 15 & & 9 weeks & 47 & 61.9 & 28.2 & 45.6 & 7.78 & 58.6 & 14.6 & 8.45 & 0.040 & 2.811 & 15 \\
\hline
\end{tabular}


Table VIII. Physical and Chemical Measurements on Dog No. 19 (Mean Weight, 18.0 Kilograms)

\begin{tabular}{|c|c|c|c|c|c|c|c|c|c|c|c|c|c|c|c|c|c|}
\hline & & $\begin{array}{c}\text { Pre- or } \\
\text { post- } \\
\text { hemor- } \\
\text { rhagic } \\
\text { time } \\
\text { inter- } \\
\text { val }\end{array}$ & $\begin{array}{c}\text { Blood } \\
\text { re- } \\
\text { moved, } \\
\text { in } \\
\text { cc. }\end{array}$ & $\begin{array}{c}\text { Total } \\
\text { blood } \\
\text { vol- } \\
\text { ume, } \\
\text { in cc. } \\
\text { per } \\
\mathrm{kg} .\end{array}$ & $\begin{array}{c}\text { Cell } \\
\text { vol- } \\
\text { ume, } \\
\text { in } \\
\text { cc. } \\
\text { per } \\
\text { kg. }\end{array}$ & $\begin{array}{l}\text { Cell } \\
\text { vol- } \\
\text { ume } \\
\text { per } \\
\text { cent }\end{array}$ & $\begin{array}{c}\text { Red } \\
\text { cell } \\
\text { count, } \\
\text { in } \\
\text { mil- } \\
\text { lions }\end{array}$ & $\begin{array}{c}\text { Red } \\
\text { cell } \\
\text { diam- } \\
\text { eter, } \\
\text { in } \\
\text { mi- } \\
\text { crons }\end{array}$ & $\begin{array}{l}\text { Mean } \\
\text { corpus- } \\
\text { cular } \\
\text { vol- } \\
\text { ume, in } \\
\text { cubic } \\
\text { microns }\end{array}$ & $\begin{array}{l}\text { Oxygen- } \\
\text { combin- } \\
\text { ing ca- } \\
\text { pacity. } \\
\text { in } \\
\text { volumes } \\
\text { per cent }\end{array}$ & $\begin{array}{l}\text { White } \\
\text { cell } \\
\text { count, } \\
\text { in } \\
\text { thou- } \\
\text { sands }\end{array}$ & $\begin{array}{c}\text { Non- } \\
\text { protein } \\
\text { nitrogen } \\
\text { in whole } \\
\text { blood, } \\
\text { g. per } \\
100 \text { cc. }\end{array}$ & $\begin{array}{c}\text { Protein } \\
\text { nitro- } \\
\text { gen in } \\
\text { whole } \\
\text { blood, } \\
\text { g. per } \\
100 \text { cc. }\end{array}$ & $\begin{array}{l}\text { Urea } \\
\text { nitro- } \\
\text { gen in } \\
\text { whole } \\
\text { blood, } \\
\text { mg. per } \\
\text { lo0 cc. }\end{array}$ & $\begin{array}{c}\text { Amino } \\
\text { acid } \\
\text { nitrogen } \\
\text { in whole } \\
\text { blood, } \\
\text { mg. per } \\
\text { 100 cc. }\end{array}$ & $\begin{array}{c}\text { Creatin- } \\
\text { ine } \\
\text { in } \\
\text { whole } \\
\text { blood, } \\
\text { mg. per } \\
100 \text { cc. }\end{array}$ & \\
\hline 1 & Pre-hemorrhage & 30 days & 50 & 67.7 & 39.6 & 58.4 & 7.54 & 6.80 & 77.4 & 24.5 & 3.60 & 0.031 & 3.479 & 12.73 & $\ldots \ldots \ldots \ldots . .$. & 1.72 & 1 \\
\hline 2 & & 27 days & 51 & $\ldots \ldots \ldots \ldots$ & $\ldots \cdots \cdots$ & 59.8 & 7.00 & 6.90 & 85.4 & 22.8 & 4.55 & 0.037 & 3.445 & 18.02 & $\ldots . . . . . .$. & 1.72 & 2 \\
\hline 3 & & 3 days & 20 & 70.2 & 41.3 & 58.8 & $\ldots . . . . . . .$. & $\ldots-\ldots . . . .$. & 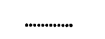 & &........- & $\ldots+\ldots . . . . . . . .$. & .................... & $\ldots . . . . .$. & & & 3 \\
\hline 4 & & same day & 49 & .............. & ............... & $\ldots \ldots \ldots \ldots . .$. & $\ldots \ldots \ldots \ldots . .$. & $\ldots . \cdots \cdots \cdots$ & $\ldots . . . . . . . .$. & 21.6 & $\ldots . . . \cdots \cdots \cdots . .$. & 0.036 & 3.284 & 19.30 & 4.05 & 1.65 & 4 \\
\hline 5 & First Hemorrhage & & 238 & $\ldots \ldots \ldots \ldots . .$. & $\ldots \ldots \ldots$ & $\ldots \ldots \ldots$ & $\ldots \ldots \ldots \ldots$ & $\ldots \ldots \cdots \cdots$ & $\ldots \cdots \cdots \cdots$ & $\ldots+\ldots . \cdots+\cdots$ & $\ldots \ldots \ldots \ldots \ldots$ & ................... & $\ldots+\cdots \cdots \cdots \cdots$ & & & & 5 \\
\hline 6 & & same day & 50 & .............. & $\ldots \ldots \ldots \ldots$ & $\ldots \ldots \ldots \ldots$ & .............. & $\ldots . . . . . . .$. & $\ldots \ldots \ldots . . . .$. & 17.8 & $\ldots . . . . . . .$. & 0.037 & 2.833 & 22.48 & 15.32 & 1.66 & 6 \\
\hline 7 & Second Hemorrhag & & 247 & $\ldots \ldots \ldots \ldots \ldots$ & $\ldots \cdots \cdots \cdots$ & $\ldots \ldots \ldots \ldots \ldots$ & 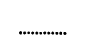 & $\ldots \ldots \ldots . . . .$. & & & $\ldots$ & & & $\ldots$ & & $\ldots \ldots \ldots$ & 7 \\
\hline 8 & Post-hemorrhage & 2 days & 49 & $\ldots \ldots \ldots \ldots$ & $\therefore$ & $\ldots \ldots \ldots$ & 5.06 & 6.88 & .............. & 16.0 & 4.15 & 0.033 & 2.617 & 18.61 & 16.73 & 1.65 & 8 \\
\hline 9 & & 4 days & 50 & 64.4 & 22.7 & 35.2 & 5.10 & 6.91 & 69.0 & 14.3 & 5.85 & 0.028 & 2.492 & 16.37 & 15.60 & 1.57 & 9 \\
\hline 10 & & 1 week & 52 & 56.1 & 22.1 & 39.3 & 4.98 & 6.84 & 78.9 & 16.4 & 5.55 & 0.036 & 2.774 & 25.07 & 17.27 & 1.63 & 10 \\
\hline 11 & & 2 weeks & 49 & 56.3 & 22.0 & 39.1 & 5.74 & 6.51 & 68.1 & 14.5 & 4.50 & 0.033 & 2.637 & 20.39 & 18.10 & 1.66 & 11 \\
\hline 12 & & 3 weeks & 51 & $\ldots+\ldots$ & $\ldots \cdots \cdots \cdots$ & $\ldots$ & 5.66 & 6.43 & $\ldots \ldots \ldots \ldots . .$. & 15.0 & 5.15 & 0.031 & 2.469 & 14.83 & 21.12 & 1.58 & 12 \\
\hline 13 & & 4 weeks & 52 & 59.7 & 21.8 & 36.6 & 6.11 & 6.12 & 59.9 & 15.2 & 4.45 & 0.038 & 2.672 & 18.80 & 16.25 & 1.55 & 13 \\
\hline 14 & & 5 weeks & 51 & 52.6 & 19.6 & 37.2 & 6.38 & 6.21 & 58.3 & 13.8 & 3.60 & 0.031 & 2.649 & 16.94 & 17.11 & 1.56 & 14 \\
\hline 15 & & 6 weeks & 52 & 50.8 & 18.6 & 36.7 & 5.88 & 6.24 & 62.4 & 12.2 & 3.90 & 0.035 & 2.305 & 18.80 & 7.08 & 1.38 & 15 \\
\hline 16 & & 7 weeks & 52 & 53.2 & 18.6 & 34.9 & 6.68 & 6.27 & 52.2 & 13.0 & 3.90 & 0.031 & 2.499 & 14.99 & 16.40 & 1.50 & 16 \\
\hline 17 & & 8 weeks & 53 & 52.5 & 18.9 & 36.1 & 7.38 & 6.10 & 48.9 & 11.8 & 2.60 & 0.024 & 2.186 & 10.75 & 6.50 & 1.49 & 17 \\
\hline 18 & & 9 weeks & 51 & 51.3 & 19.3 & 37.6 & 7.48 & 6.13 & 50.3 & 13.7 & 2.60 & 0.034 & 2.406 & 13.55 & 14.12 & 1.49 & 18 \\
\hline 19 & & 10 weeks & 50 & 48.9 & 17.9 & 36.7 & 7.62 & 5.95 & 48.2 & 12.2 & 3.50 & 0.025 & 2.345 & 3.46 & 9.98 & 1.52 & 19 \\
\hline 20 & & 11 weeks & 50 & 48.1 & 16.6 & 34.5 & 8.32 & 5.94 & 41.5 & 10.6 & 3.05 & 0.030 & 2,340 & 17.05 & 8.90 & 1.51 & 20 \\
\hline 21 & & 12 weeks & 51 & 47.1 & 16.5 & 35.1 & 8.08 & 5.87 & 43.4 & 10.8 & 3.35 & 0.029 & 2.276 & 13.21 & 16.65 & 1.52 & 21 \\
\hline
\end{tabular}


Table IX. Physical and Chemical Measurements on Dog No. 32 ㅇ (Mean Weight, 17.6 Kilograms)

\begin{tabular}{|c|c|c|c|c|c|c|c|c|c|c|c|c|c|c|c|}
\hline $\begin{array}{l}\because \\
\therefore \\
\therefore\end{array}$ & & $\begin{array}{c}\text { Pre- or } \\
\text { post- } \\
\text { hemor- } \\
\text { rhagic } \\
\text { time } \\
\text { inter- } \\
\text { val }\end{array}$ & $\begin{array}{c}\text { Blood } \\
\text { re- } \\
\text { moved, } \\
\text { in } \\
\text { cc. }\end{array}$ & $\begin{array}{c}\text { Total } \\
\text { blood } \\
\text { vol- } \\
\text { ume, } \\
\text { in cc. } \\
\text { per } \\
\mathrm{kg} .\end{array}$ & $\begin{array}{l}\text { Cell } \\
\text { vol- } \\
\text { ume, } \\
\text { in } \\
\text { cc. } \\
\text { per } \\
\text { kg. }\end{array}$ & $\begin{array}{l}\text { Cell } \\
\text { vol- } \\
\text { ume } \\
\text { per } \\
\text { cent }\end{array}$ & $\begin{array}{c}\text { Red } \\
\text { cell } \\
\text { count, } \\
\text { in } \\
\text { mil- } \\
\text { lions }\end{array}$ & $\begin{array}{l}\text { Red } \\
\text { cell } \\
\text { diam- } \\
\text { eter, } \\
\text { in } \\
\text { mi- } \\
\text { crons }\end{array}$ & $\begin{array}{l}\text { Mean } \\
\text { corpus- } \\
\text { cular } \\
\text { vol- } \\
\text { ume, in } \\
\text { cubic } \\
\text { microns }\end{array}$ & $\begin{array}{l}\text { Oxygen- } \\
\text { combin- } \\
\text { ing ca- } \\
\text { pacity, } \\
\text { in } \\
\text { volumes } \\
\text { per cent }\end{array}$ & $\begin{array}{c}\text { White } \\
\text { cell } \\
\text { count, } \\
\text { in } \\
\text { thou- } \\
\text { sands }\end{array}$ & $\begin{array}{c}\text { Non- } \\
\text { protein } \\
\text { nitrogen } \\
\text { in whole } \\
\text { blood, } \\
\text { g. per } \\
100 \mathrm{cc} .\end{array}$ & $\begin{array}{c}\text { Protein } \\
\text { nitro- } \\
\text { gen in } \\
\text { whole } \\
\text { blood, } \\
\text { g.per } \\
100 \text { cc. }\end{array}$ & $\begin{array}{l}\text { Urea } \\
\text { nitro- } \\
\text { gen in } \\
\text { whole } \\
\text { blood, } \\
\text { mg. per } \\
\text { loo cc. }\end{array}$ & \\
\hline 1 & Pre-hemorrhage & 23 days & 43 & $\ldots \ldots \ldots \ldots . . . .$. & $\ldots \ldots \ldots \ldots . . .$. & $\ldots \ldots \ldots \ldots$ & 6.74 & 7.03 & $\ldots \ldots \ldots \ldots . . .$. & 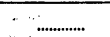 & 9.05 & 0.035 & 3.420 . & $\ldots$ & 1 \\
\hline 2 & & 18 days & 49 & 68.8 & 32.9 & 47.9 & 6.63 & 7.13 & 72.3 & 15.4 & 9.45 & 0.042 & 2.974 & $\therefore \ldots . . .$. & 2 \\
\hline 3 & & 16 days & 49 & ............... & .............: & 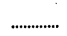 & 6.81 & 7.40 & ....................... & 19.7 & 8.35 & 0.046 & 3.437 & 7.98 & 3 \\
\hline 4 & & 14 days & 48 & 61.6 & 28.6 & 46.9 & 6.50 & 7.25 & 72.2 & 21.0 & 7.95 & 0.042 & 3.171 & 6.84 & 4 \\
\hline 5 & & 7 days & 58 & 69.0 & 34.2 & 49.6 & 7.21 & 6.93 & 68.8 & 19.7 & 7.75 & 0.032 & 3.135 & 7.40 & 5 \\
\hline 6 & & same day & 58 & $\ldots . . . . . . . .$. & $\ldots \ldots \ldots . . . .$. & $\ldots . . . . . . . .$. & .................. & $\ldots . . . . . . .$. & $\ldots \ldots \ldots$ & 14.1 & $\ldots . . . . . . . .$. & 0.037 & 2.995 & 14.25 & 6 \\
\hline 7 & First Hemorrhage & & 183 & $\ldots \ldots \ldots \ldots . . .$. & $\ldots \ldots \ldots \ldots . .$. & $\ldots \ldots \ldots \ldots . .$. & $\ldots \ldots \ldots \ldots$ & $\ldots \ldots \ldots \ldots \ldots$ & $\ldots \ldots \ldots \ldots$ & $\ldots . . . \cdots \cdots . .$. & $\ldots \ldots \ldots \ldots . .$. & $\ldots \ldots \ldots \ldots \ldots$ & $\ldots$ & $\ldots \ldots \ldots$ & 7 \\
\hline 8 & . & same day & 42 & 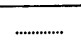 & ............. & $\ldots \ldots \ldots \ldots$ & $\ldots \ldots \ldots \ldots$ & $\ldots . . . . . . . .$. & .............. & 14.8 & $\ldots \ldots \ldots \ldots$ & 0.038 & 2.899 & 22.43 & 8 \\
\hline 9 & Second Hemorrhage & & 200 & $\ldots \ldots \ldots \ldots . .$. & $\ldots . . \cdots \cdots \cdots$ & $\ldots \ldots \ldots \ldots \ldots$ & ............... & $\ldots \ldots \ldots \ldots . .$. & $\ldots . . \cdots \cdots \cdots . . .$. & $\ldots . . \cdots \cdots \cdots . .$. & $\ldots . . \cdots \cdots \cdots \cdots$ & ................... & ................. & $\ldots . . \cdots \cdots \cdots \cdots$ & 9 \\
\hline 10 & Post-hemorrhage & 1 day & 49 & 56.6 & 19.1 & 33.8 & 4.66 & 6.77 & 72.5 & 11.8 & 8.40 & 0.036 & 2.470 & 17.89 & 10 \\
\hline 11 & & 3 days & 66 & 58.4 & 19.4 & 33.3 & 4.80 & 6.69 & 69.3 & 11.1 & 11.80 & 0.033 & 2.573 & 17.76 & 11 \\
\hline 12 & & 5 days & 50 & 57.6 & 19.2 & 33.8 & 4.73 & 6.86 & 71.4 & 11.3 & 10.15 & 0.032 & 2.472 & 15.35 & 12 \\
\hline 13 & & I week & 55 & 60.1 & 18.7 & 31.1 & 4.76 & 6.70 & 65.3 & 11.1 & 7.95 & 0.031 & 2.445 & 9.75 & 13 \\
\hline 14 & & 2 weeks & 47 & 57.6 & 19.5 & 33.8 & 5.09 & 6.26 & 67.5 & 11.0 & 7.70 & 0.033 & 2.323 & 19.61 & 14 \\
\hline 15 & $\therefore \cdot$ & 3 weeks & 50 & 57.1 & 19.0 & 33.6 & 4.82 & 6.37 & 69.7 & 11.1 & 9.00 & 0.032 & 2.411 & 22.46 & 15 \\
\hline 16 & & 4 weeks & 62 & 59.0 & 21.3 & 36.2 & 5.64 & 6.49 & 64.2 & 11.9 & 6.95 & 0.034 & 2.444 & $\ldots \ldots \ldots$ & 16 \\
\hline 17 & & 5 weeks & 51 & 57.1 & 19.9 & 34.9 & 5.20 & 6.44 & 67.1 & 12.3 & 7.45 & 0.030 & 2.520 & 16.86 & 17 \\
\hline 18 & & 6 weeks & 51 & 56.4 & 20.4 & 36.1 & 5.99 & 6.28 & 60.3 & 12.3 & 8.90 & 0.034 & 2.567 & 11.27 & 18 \\
\hline 19 & & 7 weeks & 41 & 58.3 & 20.3 & 34.9 & 5.80 & 6.53 & 60.2 & 13.1 & 11.10 & $\ldots . . . . . . . . . . . .$. & ..................: & $\ldots$ & 19 \\
\hline 20 & & 9 weeks & 35 & 60.0 & 21.7 & 36.3 & 5.78 & 6.54 & 62.8 & 13.4 & 7.10 & $\ldots . . . . . . . . . . . .$. & 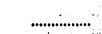 & $\ldots \ldots \ldots . . . . . . .$. & 20 \\
\hline 21 & & 11 weeks & 54 & 58.9 & 22.4 & 38.1 & 6.19 & 6.45 & 61.6 & 13.9 & 6.75 & 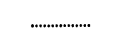 & ................. & .................' & 21 \\
\hline 22 & & 12 weeks & 38 & 57.5 & 21.5 & 37.5 & 5.98 & 6.50 & 62.7 & 13.8 & 6.75 & $\ldots . . . . . . . . . . . .$. & $\ldots . . . . . . . . . . .$. & $\ldots \ldots . . . . . . .$. & 22 \\
\hline
\end{tabular}


Table X. Physical and Chemical Measurements on Dog No. $17 \delta^{\pi}$ (Mean Weight, 16.8 Kilograms)

\begin{tabular}{|c|c|c|c|c|c|c|c|c|c|c|c|c|c|c|c|c|c|}
\hline & & $\begin{array}{c}\text { Pre- or } \\
\text { post- } \\
\text { hemor- } \\
\text { rhagic } \\
\text { time } \\
\text { inter- } \\
\text { val }\end{array}$ & $\begin{array}{c}\text { Blood } \\
\text { re- } \\
\text { moved, } \\
\text { in } \\
\text { cc. }\end{array}$ & $\begin{array}{c}\text { Total } \\
\text { blood } \\
\text { vol- } \\
\text { ume, } \\
\text { in cc. } \\
\text { per } \\
\text { kg. }\end{array}$ & $\begin{array}{l}\text { Cell } \\
\text { vol- } \\
\text { ume, } \\
\text { in } \\
\text { cc. } \\
\text { per } \\
\text { kg. }\end{array}$ & $\begin{array}{l}\text { Cell } \\
\text { vol- } \\
\text { ume } \\
\text { per } \\
\text { cent }\end{array}$ & $\begin{array}{c}\text { Red } \\
\text { cell } \\
\text { count, } \\
\text { in } \\
\text { mil- } \\
\text { lions }\end{array}$ & $\begin{array}{l}\text { Red } \\
\text { cell } \\
\text { diam- } \\
\text { eter, } \\
\text { in } \\
\text { mi- } \\
\text { crons }\end{array}$ & $\begin{array}{l}\text { Mean } \\
\text { corpus- } \\
\text { cular } \\
\text { vol- } \\
\text { ume, in } \\
\text { cubic } \\
\text { microns }\end{array}$ & $\begin{array}{l}\text { Oxygen- } \\
\text { combin- } \\
\text { ing ca- } \\
\text { pacity. } \\
\text { in } \\
\text { volumes } \\
\text { per cent }\end{array}$ & $\begin{array}{l}\text { White } \\
\text { cell } \\
\text { count, } \\
\text { in } \\
\text { thou- } \\
\text { sands }\end{array}$ & $\begin{array}{c}\text { Non- } \\
\text { protein } \\
\text { nitrogen } \\
\text { in whole } \\
\text { blood, } \\
\text { g. per } \\
100 \text { cc. }\end{array}$ & $\begin{array}{c}\text { Protein } \\
\text { nitro- } \\
\text { gen in } \\
\text { whole } \\
\text { blood, } \\
\text { g.per } \\
100 \text { cc. }\end{array}$ & $\begin{array}{l}\text { Urea } \\
\text { nitro- } \\
\text { gen in } \\
\text { whole } \\
\text { blood, } \\
\text { mg. per } \\
100 \text { cc. }\end{array}$ & $\begin{array}{l}\text { Amino } \\
\text { acid } \\
\text { nitrogen } \\
\text { in whole } \\
\text { blood, } \\
\text { mg. per } \\
\text { loo cc. }\end{array}$ & $\begin{array}{l}\text { Creatin- } \\
\text { ine } \\
\text { in } \\
\text { whole } \\
\text { blood, } \\
\text { mg. per } \\
100 \text { cc. }\end{array}$ & \\
\hline 1 & Pre-hemorrhage & 94 days & 52 & 74.9 & 38.5 & 51.4 & 7.54 & 7.30 & 68.2 & 17.1 & 8.05 & $\ldots \ldots \ldots \ldots \ldots$ & $\ldots \ldots \ldots \ldots . . . .$. & 6.78 & $\ldots . . . \cdots \cdots$ & 1.41 & 1 \\
\hline 2 & & 90 days & 10 & $\ldots \ldots \ldots \ldots$ & $\ldots . . \cdots \cdots$ & $\ldots . . . \cdots \cdots . . .$. & ............. & $\ldots$ & $\ldots . . . \cdots+\cdots$ & $\ldots . . . . . . .$. & $\ldots+\ldots . . .$. & $\ldots \ldots \ldots \ldots . .$. & $\ldots \ldots \ldots \ldots . . .$. & 5.92 & $\ldots . . . \cdots \cdots . .$. & 1.43 & 2 \\
\hline 3 & & 80 days & 10 & $\ldots . . . \cdots \cdots . . .$. & $\ldots \ldots \ldots \ldots . . .$. & $\ldots \ldots \ldots \ldots . . .$. & $\ldots \ldots \ldots \ldots . .$. & $\ldots \ldots \ldots \ldots . . .$. & .................... & $\ldots . . . \cdots \cdots . . .$. & $\ldots \ldots \ldots$ & 0.028 & 3.449 & ......................... & $\ldots . . . \cdots \cdots \cdots$ & & 3 \\
\hline 4 & & 32 days & 53 & 69.8 & 32.7 & 46.8 & 7.89 & 6.90 & 59.3 & 18.9 & 5.30 & 0.030 & 3.331 & 13.74 & $\ldots \ldots \ldots \ldots . .$. & 1.44 & 4 \\
\hline 5 & & same day & 50 & ............... & $\ldots . . \cdots \cdots \cdots . . .$. & $\ldots \ldots \ldots \ldots$ & $\ldots \ldots \ldots \ldots \ldots$ & $\ldots . \cdots \cdots \cdots \cdots$ & $\ldots \ldots \ldots \ldots . .$. & 19.8 & $\ldots \ldots \ldots \ldots \ldots$ & 0.028 & 3.277 & 12.26 & 4.84 & 1.59 & 5 \\
\hline 6 & First Hemorrhage & & 240 & $\ldots \ldots \ldots \ldots \ldots$ & .............. & ............... & .............. & $\ldots \ldots \ldots \ldots . .$. & $\ldots \ldots \ldots$ & $\ldots \ldots \ldots \ldots . .$. & $\ldots \cdots \cdots \cdots . . .$. & $\ldots$ & .................. & 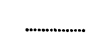 & $\ldots . \cdots \cdots \cdots . .$. & ................... & 6 \\
\hline 7 & & same day & 50 & .............. & ................... & $\cdots \cdots \cdots \cdots . .$. & $\ldots \ldots \ldots \ldots . .$. & $\ldots \ldots \ldots \cdots \cdots$ & ...................... & 18.6 & $\ldots \ldots \ldots \ldots$ & 0.030 & 3.205 & 18.48 & 5.69 & 1.66 & 7 \\
\hline 8 & Second Hemorrhag & & 225 & $\ldots \ldots \ldots \ldots . .$. & 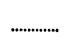 & $\ldots \ldots \ldots \ldots . .$. & $\ldots$ & $\ldots . \cdots \cdots \cdots \cdots$ & $\ldots \ldots \ldots \ldots$ & ............... & $\ldots \ldots \ldots \ldots . .$. & .................. & ................... & $\ldots$ & .............. & $\cdots \cdots \cdots$ & 8 \\
\hline 9 & Post-hemorrhage & 2 days & 80 & $\ldots \ldots \ldots \ldots . .$. & $\ldots$ & .............. & 5.73 & 6.77 & $\ldots \ldots \ldots \ldots . .$. & 13.9 & 9.45 & 0.027 & 2.523 & 14.23 & 7.34 & 1.57 & 9 \\
\hline 10 & & 5 days & 51 & 59.1 & 24.4 & 41.2 & 6.11 & 6.50 & 67.4 & 16.1 & 8.05 & 0.031 & 2.729 & 15.83 & 15.52 & 1.56 & 10 \\
\hline 11 & & 6 days & 50 & 63.7 & 22.3 & 35.0 & 6.14 & 6.60 & 57.0 & 14.4 & 7.80 & 0.027 & 2.748 & 12.92 & 6.82 & 1.54 & 11 \\
\hline 12 & & I week & 49 & 64.0 & 22.6 & 35.4 & 6.15 & 6.68 & 57.6 & 13.5 & 9.45 & 0.024 & 2.441 & 11.00 & 14.62 & 1.61 & 12 \\
\hline 13 & & 2 weeks & 50 & 64.6 & 22.9 & 35.5 & 7.10 & 6.26 & 50.0 & 14.6 & 9.55 & 0.027 & 2.843 & 13.71 & 15.92 & 1.58 & 13 \\
\hline 14 & & 3 weeks & 49 & 69.1 & 29.0 & 42.1 & 6.97 & 6.40 & 60.4 & 13.5 & 10.10 & 0.028 & 2.657 & 14.72 & 12.27 & 1.58 & 14 \\
\hline 15 & & 4 weeks & 44 & 61.7 & 23.9 & 38.7 & 7.88 & 6.07 & 49.1 & 15.1 & 5.45 & 0.026 & 2.954 & 9.11 & 7.52 & 1.55 & 15 \\
\hline 16 & & 5 weeks & 44 & 58.5 & 22.8 & 39.0 & 7.44 & 6.22 & 52.4 & 14.7 & 7.15 & 0.027 & 2.743 & 11.52 & 9.73 & 1.58 & 16 \\
\hline 17 & & 6 weeks & 60 & 65.4 & 24.4 & 37.4 & 8.04 & 6.18 & 46.5 & 14.0 & 12.00 & 0.026 & 2.764 & 10.86 & 12.97 & 1.56 & 17 \\
\hline 18 & & 7 weeks & 51 & 63.0 & 22.0 & 35.0 & 7.44 & 6.25 & 47.0 & 11.8 & 6.85 & 0.023 & 2.487 & 12.08 & 4.47 & 1.50 & 18 \\
\hline 19 & & 8 weeks & 52 & 65.3 & 22.2 & 34.0 & 7.94 & 6.08 & 42.8 & 12.1 & 7.30 & 0.026 & 2.409 & 11.19 & 3.06 & 1.50 & 19 \\
\hline 20 & & 9 weeks & 52 & 63.0 & 21.6 & 34.2 & 7.12 & 6.18 & 48.0 & 11.7 & 8.30 & 0.030 & 2.740 & 10.08 & 4.50 & 1.50 & 27 \\
\hline 21 & & 10 weeks & 53 & 67.1 & 23.6 & 35.1 & 8.03 & 6.05 & 43.7 & 11.7 & 8.10 & 0.026 & 2.474 & 5.98 & 12.67 & 1.49 & 21 \\
\hline 22 & & 11 weeks & 50 & 70.6 & 24.6 & 34.9 & 7.88 & 6.02 & 44.3 & 10.7 & 7.10 & 0.025 & 2.545 & 3.40 & 10.63 & 1.50 & 22 \\
\hline 23 & & 12 weeks & 62 & $\ldots \ldots \ldots \ldots . .$. & $\ldots . . . \cdots \cdot . .$. & $\ldots \ldots \ldots$ & 7.46 & 5.86 & 46.9 & 11.3 & 9.30 & 0.029 & 2.716 & 11.47 & 8.19 & 1.52 & 23 \\
\hline
\end{tabular}


Table XI. Physical and Chemical Measurements on Dog No. $18 \sigma^{7}$ (Mean Weight, 20.8 Kilograms)

\begin{tabular}{|c|c|c|c|c|c|c|c|c|c|c|c|c|c|c|c|c|c|}
\hline & & $\begin{array}{c}\text { Pre- or } \\
\text { post- } \\
\text { hemor- } \\
\text { rhagic } \\
\text { time } \\
\text { inter- } \\
\text { val }\end{array}$ & $\begin{array}{c}\text { Blood } \\
\text { re- } \\
\text { moved, } \\
\text { in } \\
\text { cc. }\end{array}$ & $\begin{array}{c}\text { Total } \\
\text { blood } \\
\text { vol- } \\
\text { ume, } \\
\text { in cc. } \\
\text { per } \\
\mathrm{kg} .\end{array}$ & $\begin{array}{c}\text { Cell } \\
\text { vol- } \\
\text { ume, } \\
\text { in } \\
\text { cc. } \\
\text { per } \\
\mathrm{kg} .\end{array}$ & $\begin{array}{l}\text { Cell } \\
\text { vol- } \\
\text { ume } \\
\text { per } \\
\text { cent }\end{array}$ & $\begin{array}{c}\text { Red } \\
\text { cell } \\
\text { count, } \\
\text { in } \\
\text { mil- } \\
\text { lions }\end{array}$ & $\begin{array}{l}\text { Red } \\
\text { cell } \\
\text { diam- } \\
\text { eter, } \\
\text { in } \\
\text { mi- } \\
\text { crons }\end{array}$ & $\begin{array}{l}\text { Mean } \\
\text { corpus- } \\
\text { cular } \\
\text { vol- } \\
\text { ume, in } \\
\text { cubic } \\
\text { microns }\end{array}$ & $\begin{array}{l}\text { Oxygen- } \\
\text { combin- } \\
\text { ing ca- } \\
\text { pacity, } \\
\text { in } \\
\text { volumes } \\
\text { per cent }\end{array}$ & $\begin{array}{l}\text { White } \\
\text { cell } \\
\text { count, } \\
\text { in } \\
\text { thou- } \\
\text { sands }\end{array}$ & $\begin{array}{c}\text { Non- } \\
\text { protein } \\
\text { nitrogen } \\
\text { in whole } \\
\text { blood, } \\
\text { g.per } \\
\text { loo cc. }\end{array}$ & $\begin{array}{c}\text { Protein } \\
\text { nitro- } \\
\text { gen in } \\
\text { whole } \\
\text { blood, } \\
\text { g.per } \\
100 \text { cc. }\end{array}$ & $\begin{array}{l}\text { Urea } \\
\text { nitro- } \\
\text { gen in } \\
\text { whole } \\
\text { blood, } \\
\text { mg. per } \\
100 \text { cc. }\end{array}$ & $\begin{array}{c}\text { Amino } \\
\text { acid } \\
\text { nitrogen } \\
\text { in whole } \\
\text { blood, } \\
\text { mg. per } \\
\text { loo cc. }\end{array}$ & $\begin{array}{c}\text { Creatin- } \\
\text { ine } \\
\text { in } \\
\text { whole } \\
\text { blood, } \\
\text { mg. per } \\
100 \text { cc. }\end{array}$ & \\
\hline 1 & Pre-hemorrhage & 84 days & 53 & 53.4 & 23.1 & 43.2 & 6.20 & 7.00 & 69.7 & $\ldots \ldots \ldots . . . . . .$. & 3.15 & 0.026 & 2.875 & $\ldots \ldots \ldots \ldots . . . .$. & $\ldots . . . . . . . . . .$. & 1.42 & 1 \\
\hline 2 & & 61 days & 15 & $\ldots . . . . . . . .$. & $\ldots \ldots \ldots . . . .$. & $\ldots . . . . . . . .$. & $\ldots . . . . . . . .$. & $\ldots \ldots \ldots . . . .$. & $\ldots . . . \cdots \ldots . .$. & $\ldots . . . . . . . .$. & $\ldots . . . . . . . .$. & 0.025 & 2.831 & 8.79 & ................... & 1.48 & 2 \\
\hline 3 & & 54 days & 15 & $\ldots . . . \ldots . . . .$. & ................... & $\ldots \ldots \ldots \ldots . . .$. & $\ldots \ldots \ldots \ldots$ & $\ldots \ldots \ldots . . . .$. & $\ldots \ldots \ldots$ & ................... & $\ldots . . . . . . . .$. & $\ldots$ & $\cdots$ & 7.80 & .................... & 1.43 & 3 \\
\hline 4 & & 33 days & 15 & ................ & ............... & $\ldots . . . \cdots \cdots . . .$. & $\ldots . . . . . . . .$. & 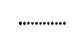 & $\ldots \ldots \ldots \ldots$ & $\ldots \ldots \ldots . . . . .$. & $\ldots \ldots \ldots . . . .$. & $\ldots \ldots \ldots . . . . . .$. & & 5.74 & ..................... & 1.49 & 4 \\
\hline 5 & & 10 days & 50 & 53.4 & 25.8 & 48.4 & 7.07 & 6.90 & 68.5 & 17.4 & 6.75 & 0.034 & 3.012 & 13.55 & ................... & 1.64 & 5 \\
\hline 6 & & 7 days & 52 & 58.4 & 26.9 & 46.1 & 6.99 & 6.80 & 66.0 & 17.3 & 5.10 & 0.030 & 3.387 & 13.79 & ...................... & 1.81 & 6 \\
\hline 7 & & same day & 51 & $\ldots . . . . . . . .$. & $\ldots \ldots \ldots . . . .$. & $\ldots \ldots \ldots \ldots . .$. & $\ldots \ldots \ldots \ldots$ & $\ldots \ldots \ldots \ldots$ & $\ldots \ldots \ldots \ldots$ & 17.4 & $\ldots \ldots \ldots$ & 0.031 & 3.431 & 12.32 & $\ldots . . . . . . . . .$. & 1.76 & 7 \\
\hline 8 & First Hemorrhage & & 210 & ............... & ...................... & $\ldots \ldots \ldots \ldots . .$. & $\ldots \ldots \ldots . .$. & $\ldots \ldots \ldots \ldots$ & ……..... & .............. & 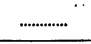 & ................. & …….......... & $\ldots \ldots \ldots \ldots$ & …….......... & ................. & 8 \\
\hline 9 & & same day & 50 & $\ldots \ldots \ldots . . .$. & ................... & $\ldots \ldots \ldots \ldots . .$. & $\ldots \ldots \ldots \ldots$ & $\ldots \ldots \ldots \ldots$ & $\ldots \ldots \ldots \ldots$ & 14.1 & $\ldots . . . . . . . .$. & 0.031 & 2.454 & 19.64 & $\ldots \ldots \ldots . . .$. & 1.64 & 9 \\
\hline 10 & Second Hemorrhac & & 241 & $\ldots \ldots \ldots \ldots . .$. & ............... & $\ldots . . \cdots \cdots \cdots \cdots$ & $\ldots . \cdots \cdots \cdots \cdots$ & .............. & $\ldots \ldots \ldots \ldots . .$. & ............... & .............. & .................. & $\ldots \ldots \cdots \cdots \cdots \cdots$ & $\ldots+\cdots \cdots \cdots \cdots \cdots \cdot \cdots$ & $\ldots \ldots \cdots \cdots \cdots$ & $\ldots \cdots \cdots \cdots$ & 10 \\
\hline 11 & Post-hemorrhage & 1 day & 51 & 54.9 & 16.8 & 30.6 & 5.24 & 6.57 & 58.4 & 12.7 & 7.55 & 0.028 & 2.412 & 15.36 & $\ldots \ldots \ldots \ldots$ & 1.65 & 11 \\
\hline 12 & & 3 days & 45 & 49.5 & 16.4 & 33.0 & 4.67 & 6.61 & 70.7 & 9.8 & 6.65 & 0.030 & 2.195 & 19.15 & 4.94 & 1.68 & 12 \\
\hline 13 & & 5 days & 50 & 50.7 & 17.4 & 34.2 & 4.48 & 6.35 & 76.3 & $\ldots \ldots \ldots \ldots . . .$. & 8.80 & 0.029 & 2.286 & 13.89 & $\ldots . . . \cdots \cdots$ & 1.69 & 13 \\
\hline 14 & & 1 week & 45 & 50.0 & 16.6 & 33.3 & 4.54 & 6.50 & 73.4 & $\ldots \ldots \ldots$ & 6.80 & 0.030 & 2.285 & 17.17 & 3.61 & 1.74 & 14 \\
\hline 15 & & 2 weeks & 48 & 48.5 & 17.8 & 35.5 & 6.34 & 6.28 & 56.0 & 10.4 & 7.20 & 0.029 & 2.236 & 17.35 & 8.74 & 1.63 & 15 \\
\hline 16 & & 3 weeks & 50 & 56.8 & 19.2 & 33.8 & 6.20 & 6.22 & 54.5 & 11.0 & 8.70 & 0.029 & 2.336 & 14.83 & 10.56 & 1.70 & 16 \\
\hline 17 & & 4 weeks & 49 & 60.3 & 22.1 & 36.6 & 6.48 & 6.37 & 56.5 & 10.5 & 5.80 & 0.027 & 2.298 & 12.82 & 12.86 & 1.70 & 17 \\
\hline 18 & & 5 weeks & 72 & 54.2 & 19.3 & 35.7 & 6.48 & 6.21 & 55.1 & 11.6 & 6.50 & 0.029 & 2.396 & 14.21 & 13.11 & 1.66 & 18 \\
\hline 19 & & 6 weeks & 50 & 53.2 & 18.1 & 34.1 & 6.58 & 6.20 & 51.8 & 11.4 & 4.25 & 0.028 & 2.262 & 12.77 & 20.98 & 1.70 & 19 \\
\hline 20 & & 7 weeks & 50 & 59.3 & 21.0 & 35.3 & 6.45 & 6.02 & 54.7 & 11.4 & 7.40 & 0.028 & 2.337 & 10.44 & 18.93 & 1.67 & 20 \\
\hline 21 & & 8 weeks & 49 & 57.4 & 21.5 & 37.5 & 7.10 & 5.87 & 52.8 & 11.2 & 4.65 & 0.030 & 2.545 & 9.71 & 9.03 & 1.72 & 21 \\
\hline 22 & & 9 weeks & 37 & $\ldots . . . . . . . . .$. & $\ldots . . . . . . . .$. & ............... & 7.01 & 6.08 & $\ldots . . . . . . . .$. & 10.8 & 3.90 & 0.030 & 2.285 & 17.27 & 8.00 & 1.63 & 22 \\
\hline 23 & & 10 weeks & 57 & 53.6 & 19.2 & 35.9 & 7.85 & 5.94 & 45.7 & 11.5 & 4.90 & 0.027 & 2.358 & 12.53 & 6.73 & 1.65 & 23 \\
\hline 24 & & 11 weeks & 54 & 57.9 & 18.6 & 32.1 & 7.30 & 5.96 & 44.0 & 11.2 & 3.75 & 0.028 & 2.182 & 10.65 & 3.51 & 1.67 & 24 \\
\hline 25 & & 12 weeks & 53 & 56.1 & 20.1 & 35.8 & 7.68 & 5.87 & 46.6 & 10.5 & 5.40 & 0.024 & 2.341 & 6.89 & 10.42 & 1.70 & 25 \\
\hline
\end{tabular}


Table XII. Physical and Chemical Measurements on Dog No. $30 \sigma^{-1}$ (Mean Weight, 17.0 Kilograms)

\begin{tabular}{|c|c|c|c|c|c|c|c|c|c|c|c|c|c|c|c|}
\hline & & $\begin{array}{c}\text { Pre-or } \\
\text { post- } \\
\text { hemor- } \\
\text { rhagic } \\
\text { time } \\
\text { inter- } \\
\text { val }\end{array}$ & $\begin{array}{c}\text { Blood } \\
\text { re- } \\
\text { moved, } \\
\text { in } \\
\text { cc. }\end{array}$ & $\begin{array}{c}\text { Total } \\
\text { blood } \\
\text { vol- } \\
\text { ume, } \\
\text { in cc. } \\
\text { per } \\
\mathrm{kg} .\end{array}$ & $\begin{array}{l}\text { Cell } \\
\text { vol- } \\
\text { ume, } \\
\text { in } \\
\text { cc. } \\
\text { per } \\
\text { kg. }\end{array}$ & $\begin{array}{l}\text { Cell } \\
\text { vol- } \\
\text { ume } \\
\text { per } \\
\text { cent }\end{array}$ & $\begin{array}{c}\text { Red } \\
\text { cell } \\
\text { count, } \\
\text { in } \\
\text { mil- } \\
\text { lions }\end{array}$ & $\begin{array}{l}\text { Red } \\
\text { cell } \\
\text { diam- } \\
\text { eter, } \\
\text { in } \\
\text { mi- } \\
\text { crons }\end{array}$ & $\begin{array}{l}\text { Mean } \\
\text { corpus- } \\
\text { cular } \\
\text { vol- } \\
\text { ume, in } \\
\text { cubic } \\
\text { microns }\end{array}$ & $\begin{array}{c}\text { Oxyren- } \\
\text { combin- } \\
\text { ing ca- } \\
\text { pacity, } \\
\text { in } \\
\text { volumes } \\
\text { per cent }\end{array}$ & $\begin{array}{l}\text { White } \\
\text { cell } \\
\text { count, } \\
\text { in } \\
\text { thou- } \\
\text { sands }\end{array}$ & $\begin{array}{c}\text { Non- } \\
\text { protein } \\
\text { nitrogen } \\
\text { in whole } \\
\text { blood, } \\
\text { g.per } \\
100 \text { cc. }\end{array}$ & $\begin{array}{c}\text { Protein } \\
\text { nitro- } \\
\text { gen in } \\
\text { whole } \\
\text { blood, } \\
\text { g.per } \\
100 \text { cc. }\end{array}$ & $\begin{array}{c}\text { Urea } \\
\text { nitro- } \\
\text { gen in } \\
\text { whole } \\
\text { blood, } \\
\text { ma. per } \\
100 \text { cc. }\end{array}$ & \\
\hline 1 & Pre-hemorrhage & 14 days & 50 & $\ldots \ldots \ldots \ldots$ & $\ldots \ldots \ldots \ldots$ & $\ldots \ldots \ldots \ldots$ & 7.19 & 7.03 & $\ldots \ldots \ldots \ldots$ & $\ldots \ldots \ldots \ldots$ & 10.40 & 0.052 & 3.191 & $\ldots . . . . . . .$. & 1 \\
\hline 2 & & 12 days & 41 & $\ldots \ldots \ldots . .$. & $\ldots \ldots \ldots$ & $\ldots \ldots \ldots \ldots . .$. & 7.08 & 6.73 & $\ldots \ldots \ldots$ & $\ldots \ldots \ldots \ldots$ & 11.35 & 0.047 & 3.303 & 9.18 & 2 \\
\hline 3 & & 7 days & 49 & 75.9 & 32.3 & 42.6 & 6.31 & 7.04 & 67.5 & $\ldots \ldots \ldots \ldots$ & 9.95 & 0.046 & 3.338 & 6.15 & 3 \\
\hline 4 & & 5 days & 51 & 75.6 & 29.7 & 39.3 & $6 . C 0$ & 7.06 & 65.5 & 17.6 & 9.65 & 0.041 & 2.697 & 7.28 & 4 \\
\hline 5 & & 2 days & 49 & 75.4 & 29.4 & 39.0 & 6.14 & 7.03 & 63.5 & 17.3 & 8.80 & 0.035 & 2.938 & 8.88 & 5 \\
\hline 6 & & same day & 50 & $\ldots \ldots \ldots$ & $\ldots \ldots \ldots \ldots . .$. & $\ldots \ldots \ldots$ & $\ldots \ldots \ldots \ldots$ & $\ldots \cdots \cdots \cdots \cdots$ & $\ldots \ldots \ldots \ldots . .$. & 17.2 & $\ldots \ldots \ldots \ldots$ & 0.034 & 2.674 & 7.46 & 6 \\
\hline 7 & First Hemorrhage & & 220 & $\ldots . . . . . . . .$. & .............. & ............... & ............. & 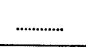 & $\ldots \ldots \ldots \ldots \ldots$ & $\ldots \ldots \ldots \ldots \ldots$ & $\ldots \ldots \ldots \ldots$ & $\ldots \ldots \ldots \ldots \ldots$ & $\ldots \ldots \ldots \ldots . . .$. & $\ldots \ldots \ldots \ldots$ & 7 \\
\hline 8 & & same day & 50 & $\ldots \ldots \ldots \ldots$ & 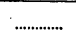 & $\ldots \ldots \ldots$ & $\ldots \ldots \ldots$ & $\ldots \ldots \ldots$ & $\ldots \ldots$ & 15.4 & $\ldots \ldots \ldots \ldots$ & 0.027 & 2.584 & 6.40 & 8 \\
\hline 9 & Second Hemorrhage & & 215 & $\ldots . . . \cdots \cdots$ & $\ldots \ldots \ldots \ldots$ & $\ldots \ldots \ldots \ldots$ & $\ldots \ldots \ldots \ldots$ & $\ldots \ldots \ldots$ & $\ldots . . . . . . .$. & 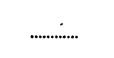 & $\ldots \ldots \ldots$ & $\ldots \ldots \ldots \ldots$ & $\ldots \ldots \ldots \ldots \ldots . . .$. & & 9 \\
\hline 10 & Post-hemorrhage & 1 day & 51 & 72.0 & 22.5 & 31.2 & 5.35 & 7.03 & 58.3 & 10.5 & 8.75 & 0.032 & 2.355 & 6.82 & 10 \\
\hline 11 & & 3 days & 50 & 74.4 & 22.8 & 30.6 & 5.19 & 6.84 & 58.9 & 12.0 & 6.80 & 0.035 & 2.505 & 6.86 & 11 \\
\hline 12 & & 5 days & 51 & 72.6 & 25.1 & 34.6 & 4.86 & 6.76 & 71.2 & 11.6 & 7.15 & 0.036 & 2.473 & 6.89 & 12 \\
\hline 13 & & 1 week & 89 & 73.3 & 24.3 & 33.2 & 5.31 & 6.72 & $\ldots \ldots \ldots$ & 13.0 & 5.25 & 0.039 & 2.118 & 17.78 & 13 \\
\hline 14 & & 2 weeks & 49 & 78.1 & 29.3 & 37.6 & 5.99 & 6.77 & 62.8 & 13.2 & 17.00 & 0.038 & 2.224 & 25.53 & 14 \\
\hline 15 & . & 3 weeks & 49 & 78.8 & 31.6 & 40.0 & 5.93 & 6.70 & 67.4 & 12.9 & 8.80 & 0.036 & 2.820 & 21.33 & 15 \\
\hline 16 & & 4 weeks & 50 & 75.4 & 32.1 & 42.6 & 6.50 & 6.60 & 65.5 & 15.6 & 5.70 & 0.034 & 2.944 & 21.55 & 16 \\
\hline 17 & & 5 weeks & 49 & 85.6 & 38.8 & 45.4 & 7.08 & 6.56 & 64.1 & 15.7 & 7.85 & 0.033 & 3.053 & 21.88 & 17 \\
\hline 18 & & 6 weeks & 50 & 79.9 & 33.5 & 43.0 & 6.62 & 6.55 & 64.9 & 14.8 & 6.50 & 0.032 & 2.788 & 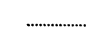 & 18 \\
\hline 19 & & 8 weeks & 49 & 85.2 & 38.4 & 45.1 & 7.32 & 6.59 & 61.6 & 16.2 & 6.55 & 0.034 & 2.939 & 13.64 & 19 \\
\hline 20 & & 10 weeks & 35 & 77.4 & 34.6 & 44.7 & 6.30 & 6.66 & 70.9 & 17.9 & 5.15 & 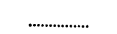 & $\ldots$ & $\ldots$ & 20 \\
\hline 21 & & 12 weeks & 38 & 74.8 & 34.6 & 46.3 & 7.40 & 6.51 & 62.5 & 16.9 & 6.55 & …….................. & $\ldots$ & $\ldots \ldots \ldots \ldots$ & 21 \\
\hline
\end{tabular}


Table XIII. Physical and Chemical Measurements on Dog No. $31 \delta^{t}$ (Mean Weight, 21.1 Kilograms)

\begin{tabular}{|c|c|c|c|c|c|c|c|c|c|c|c|c|c|c|c|}
\hline & & $\begin{array}{c}\text { Pre-or } \\
\text { post- } \\
\text { hemor- } \\
\text { rhagic } \\
\text { time } \\
\text { inter- } \\
\text { val }\end{array}$ & $\begin{array}{c}\text { Blood } \\
\text { re- } \\
\text { moved, } \\
\text { in } \\
\text { cc. }\end{array}$ & $\begin{array}{c}\text { Total } \\
\text { blood } \\
\text { vol- } \\
\text { ume, } \\
\text { in cc. } \\
\text { per } \\
\mathrm{kg} .\end{array}$ & $\begin{array}{c}\text { Cell } \\
\text { vol- } \\
\text { ume, } \\
\text { in } \\
\text { cc. } \\
\text { per } \\
\text { kg. }\end{array}$ & $\begin{array}{l}\text { Cell } \\
\text { vol- } \\
\text { ume } \\
\text { per } \\
\text { cent }\end{array}$ & $\begin{array}{c}\text { Red } \\
\text { cell } \\
\text { count, } \\
\text { in } \\
\text { mil- } \\
\text { lions }\end{array}$ & $\begin{array}{l}\text { Red } \\
\text { cell } \\
\text { diam. } \\
\text { eter, } \\
\text { in } \\
\text { mi- } \\
\text { crons }\end{array}$ & $\begin{array}{l}\text { Mean } \\
\text { corpus- } \\
\text { cular } \\
\text { vol- } \\
\text { ume, in } \\
\text { cubic } \\
\text { microns }\end{array}$ & $\begin{array}{l}\text { Oxygen- } \\
\text { combin- } \\
\text { ing ca- } \\
\text { pacity, } \\
\text { in } \\
\text { volumes } \\
\text { per cent }\end{array}$ & $\begin{array}{l}\text { White } \\
\text { cell } \\
\text { count, } \\
\text { in } \\
\text { thou- } \\
\text { sands }\end{array}$ & $\begin{array}{c}\text { Non- } \\
\text { protein } \\
\text { nitrogen } \\
\text { in whole } \\
\text { blood, } \\
\text { g. per } \\
\text { loo cc. }\end{array}$ & $\begin{array}{c}\text { Protein } \\
\text { nitro- } \\
\text { gen in } \\
\text { whole } \\
\text { blood, } \\
\text { g.per } \\
100 \mathrm{cc} .\end{array}$ & $\begin{array}{c}\text { Urea } \\
\text { nitro- } \\
\text { gen in } \\
\text { whole } \\
\text { blood, } \\
\text { mg. per } \\
\text { loo cc. }\end{array}$ & \\
\hline 1 & Pre-hemorrhage & 17 days & 50 & 81.5 & 39.4 & 48.4 & 6.89 & 7.08 & 70.3 & 16.3 & 10.00 & 0.045 & 3.517 & $\ldots \ldots \ldots \ldots \ldots$ & 1 \\
\hline 2 & & 14 days & 49 & 74.4 & 36.1 & 48.6 & 6.33 & 7.26 & 76.8 & 15.4 & 10.75 & 0.044 & 3.309 & 8.43 & 2 \\
\hline 3 & & 7 days & 49 & 72.2 & 32.8 & 45.5 & 6.54 & 7.23 & 69.6 & 19.2 & 8.05 & 0.043 & 3.144 & 7.54 & 3 \\
\hline 4 & & 5 days & 50 & 71.4 & 30.4 & 42.6 & 6.46 & 7.25 & 65.9 & 18.7 & 10.45 & 0.035 & 3.318 & 7.68 & 4 \\
\hline 5 & & same day & 50 & $\ldots \ldots \ldots$ & $\ldots \ldots \cdots \cdots \cdots$ & $\ldots \ldots \ldots \ldots$ & $\cdots \cdots \cdots \cdots$ & $\ldots \ldots \ldots \ldots$ & $\ldots . . \cdots \cdots$ & 12.5 & $\ldots \ldots \ldots \ldots \ldots$ & 0.039 & 2.883 & 14.76 & 5 \\
\hline 6 & First Hemorrhage & & 282 & 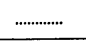 & $\ldots \ldots \ldots \ldots \ldots$ & $\ldots \ldots \ldots \ldots . .$. & .............. & $\ldots \ldots \ldots \ldots \ldots$ & .............. & ............. & .................. & ………...... & .................... & ................. & 6 \\
\hline 7 & & same day & 50 & $\ldots \ldots \ldots \ldots$ & $\ldots \ldots \ldots \ldots$ & $\ldots \cdots \cdots \cdots \cdots$ & $\ldots \ldots \ldots \ldots \ldots$ & .............. & $\ldots \ldots \ldots \ldots$ & 11.4 & $\ldots \ldots \ldots \ldots \ldots$ & 0.033 & 2.563 & 7.59 & 7 \\
\hline 8 & Second Hemorrhage & & 282 & 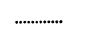 & $\ldots \ldots \ldots \ldots$ & $\ldots \ldots \ldots \ldots$ & $\ldots \ldots \ldots \ldots$ & $\ldots \ldots \ldots \ldots . .$. & .............. & $\ldots \ldots \ldots \ldots . .$. & $\ldots \ldots \ldots \ldots \cdots \cdots$ & $\ldots \ldots \ldots \cdots \cdots \cdots$ & $\ldots \ldots \ldots \cdots \cdots$ & $\ldots \ldots \ldots \ldots \ldots$ & 8 \\
\hline 9 & Post-hemorrhage & 1 day & 50 & 62.0 & 20.4 & 32.9 & 4.45 & 6.96 & 73.9 & 11.9 & 8.60 & 0.036 & 2.583 & 20.51 & 9 \\
\hline 10 & & 3 days & 49 & 67.1 & 23.6 & 35.2 & 4.59 & 7.04 & 76.7 & 10.5 & 9.10 & 0.037 & 2.934 & 17.62 & 10 \\
\hline 11 & & 5 days & 49 & 67.9 & 24.5 & 36.0 & 4.85 & 6.84 & 74.3 & 11.3 & 10.80 & 0.043 & 2.696 & 18.43 & 11 \\
\hline 12 & & 1 week & 52 & 69.3 & 24.6 & $35: 5$ & 4.10 & 6.90 & $\ldots \ldots \ldots \ldots$ & 11.4 & 8.25 & 0.039 & 2.047 & 25.02 & 12 \\
\hline 13 & & 2 weeks & 50 & 70.6 & 27.6 & 38.9 & 5.64 & 6.70 & 69.0 & 12.6 & 8.40 & 0.038 & 2.706 & 16.55 & 13 \\
\hline 14 & & 3 weeks & 48 & 70.6 & 28.9 & 40.9 & 6.34 & 6.65 & 64.5 & 13.4 & 7.50 & 0.037 & 2.804 & 30.65 & 14 \\
\hline 15 & & 4 weeks & 52 & 72.0 & 31.7 & 44.1 & 6.14 & 6.67 & 71.8 & 14.0 & 8.65 & 0.035 & 2.892 & 23.99 & 15 \\
\hline 16 & & 5 weeks & 51 & 75.3 & 34.8 & 46.3 & 6.73 & 6.67 & 68.8 & 16.9 & 8.15 & 0.036 & 2.950 & ................... & 16 \\
\hline 17 & & 6 weeks & 48 & 71.1 & 30.7 & 43.2 & 6.80 & 6.81 & 63.6 & 15.9 & 8.10 & 0.038 & 2.956 & 31.91 & 17 \\
\hline 18 & & 8 weeks & 51 & 76.3 & 32.5 & 43.2 & 6.48 & 6.91 & 66.6 & 16.5 & 7.30 & 0.038 & 3.078 & 11.52 & 18 \\
\hline 19 & & 10 weeks & 37 & 72.6 & 32.2 & 44.3 & 6.53 & 6.72 & 67.9 & 16.3 & 7.55 & 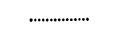 & $\ldots$ & $\ldots . . . \cdots \cdots \cdot \cdots \cdot \cdots$ & 19 \\
\hline 20 & & 12 weeks & 38 & 70.6 & 32.5 & 46.1 & 6.39 & 6.59 & 72.1 & 15.2 & 9.00 & $\ldots \ldots \ldots \ldots \ldots$ & 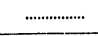 & .................. & 20 \\
\hline
\end{tabular}

